University of Louisville

ThinkIR: The University of Louisville's Institutional Repository

Electronic Theses and Dissertations

$12-2016$

\title{
The role and regulation of inhibitor of Kappa B Kinase in the impaired monocyte response.
}

Norman James Galbraith

University of Louisville

Follow this and additional works at: https://ir.library.louisville.edu/etd

Part of the Surgery Commons

\section{Recommended Citation}

Galbraith, Norman James, "The role and regulation of inhibitor of Kappa B Kinase in the impaired monocyte response." (2016). Electronic Theses and Dissertations. Paper 2576.

https://doi.org/10.18297/etd/2576

This Doctoral Dissertation is brought to you for free and open access by ThinkIR: The University of Louisville's Institutional Repository. It has been accepted for inclusion in Electronic Theses and Dissertations by an authorized administrator of ThinkIR: The University of Louisville's Institutional Repository. This title appears here courtesy of the author, who has retained all other copyrights. For more information, please contact thinkir@louisville.edu. 
THE ROLE AND REGULATION OF INHIBITOR OF KAPPA B KINASE IN THE IMPAIRED MONOCYTE RESPONSE

\author{
By \\ Norman James Galbraith \\ BSc (MedSci) University of Glasgow 2008 \\ MBChB University of Glasgow 2010 \\ MRCS Royal College of Surgeons of Edinburgh 2013 \\ MSc University of Edinburgh 2013
}

\author{
A Dissertation \\ Submitted to the Faculty of the \\ School of Medicine of the University of Louisville \\ In Partial Fulfillment of the Requirements \\ For the Degree of
}

Doctor of Philosophy in Physiology DQGBiophysics

Department of Physiology DGG\%RSK VFV

University of Louisville

Louisville, Kentucky

December 2016 
Copyright 2016 by Norman James Galbraith

All rights reserved 

THE ROLE AND REGULATION OF INHIBITOR OF KAPPA B KINASE IN THE IMPAIRED MONOCYTE RESPONSE

\author{
By \\ Norman Galbraith \\ BSc (MedSci) - University of Glasgow, Scotland 2008 \\ MBChB - University of Glasgow, Scotland 2010 \\ MRCS(Ed) - Royal College of Surgeons of Edinburgh, Scotland 2013 \\ MSc - University of Edinburgh, Scotland 2013 \\ A Dissertation Approved on
}

November 10th, 2016

By the following Dissertation Committee

Dissertation Director: Hiram C. Polk, Jr. MD

Susan Galandiuk, MD

Irving G. Joshua, PhD

Aruni Bhatnagar, PhD

Claudio Maldonado, PhD

Dale Schuschke, PhD 


\section{ACKNOWLEDGEMENTS}

I would firstly like to thank my parents, Jim and Doleen, for their faith and support throughout my time in Louisville, and for being the best role models I could hope for.

I would like to thank my mentor, Hiram C. Polk, Jr. MD, for the unique opportunity to study under his excellent guidance and supervision. The time and effort that he has invested in my development has been incredible, tirelessly pushing me to always improve. Thanks to his mentorship, I believe I have developed not only as a researcher, but as a doctor and a person. In addition, I owe my gratitude to Dr. Susan Galandiuk for her continual input and intelligent critique that improved the quality of my work over the last few years, and for the opportunity to study in the Price Institute of Surgical Research. This opportunity would not have been possible were it not for Professor Steve Wigmore, my mentor in Edinburgh, whom I am indebted to for kindly suggesting me as a candidate for this research fellowship.

I have been incredibly lucky to have the help of Sarah Gardner and Samuel Walker, whom none of this work would have been possible were it not for their hard work and dedication. In addition, I owe my gratitude to Dr. Jane V. Carter and Robert Eichenberger, along with many other students such as Campbell Bishop and Stephen Manek, whom have helped the progress of 
these experiments from concept to execution. My predecessors, Dr. Motaz Qadan and Dr. Adrian Billeter, for offering invaluable advice during my time in at the Price Institute, as well as acting as highly successful role models for what can be achieved.

I am thankful for my PhD committee, Dr. Irving Joshua, Dr. Dale Schuschke, Dr. Claudio Maldonado and Dr. Aruni Bhatnagar for their critique and insightful suggestions. In addition, they have also provided helpful contacts, such as Patrick Trainor, whose statistical expertise have elevated the analyses and presentation of the data.

Finally, I would like to thank the Price Institute of Surgical Research, founded by John W. Price and Barbara Thruston Atwood Price, and the ongoing support from Ms. Catherine Bass, who have initiated and maintained a fantastic training ground for surgical researchers of the future. 


\begin{abstract}
THE ROLE AND REGULATION OF INHIBITOR OF KAPPA B KINASE (IKK) IN THE IMPAIRED MONOCYTE RESPONSE
\end{abstract}

Norman J.Galbraith

November $10^{\text {th }}, 2016$

Trauma, major surgery and systemic infection can lead to a subsequent immunosuppressed state which can predispose patients to nosocomial infection and death. Approximately a sixth of patients will have impaired monocyte function, as determined by decreased HLA-DR expression or decreased TNF-a production in response to ex-vivo lipopolysaccharide (LPS) stimulation. This project aimed to develop an in-vitro model of impaired monocyte function using isolated monocytes from healthy volunteers, to study the effect of monocyte impairment on IKK signaling, and determine the role of IKK in the impaired monocyte response. These studies have provided the following results:

1. Monocytes treated with low dose LPS $(10 \mathrm{ng} / \mathrm{mL})$ exposure exhibit an impaired inflammatory response to a subsequent $100 \mathrm{ng} / \mathrm{mL}$ LPS challenge. Levels of TNF- $\alpha$ and IL-10 production, as well as monocyte HLA-DR expression, were decreased in the impaired monocyte compared with its naïve counterpart. 
2. Impaired monocytes that exhibited this impaired response were found to have decreased NFKB activation. In addition, while total levels of IKK- $\alpha$ and IKK- $\beta$ were similar between naïve and impaired conditions, decreased IKK phosphorylation was observed in the impaired monocyte.

3. Loss of IKK function studies using IKK-16 (a specific IKK inhibitor) showed that decreases in both TNF- $\alpha$ and IL-10 production were attributable to decreased IKK activation, demonstrating a causal relationship between $\mathrm{IKK}$ function and the monocyte cytokine response. However, HLA-DR expression was not dependent on IKK function, suggesting that other signaling pathways may also be altered in monocyte impairment that may affect HLA-DR expression.

4. The impaired monocyte phenotype also results in decreased chemokine production (such as MCP-1 and IP-10) and growth factor secretion (such as GM-CSF).

Our data highlight the importance of IKK function in the host defense response to infective stimuli, but also demonstrate the effects of negative feedback regulation following a primary stimulus. Excessive IKK stimulation, or indeed suppression of IKK activity, is likely to be detrimental to the surgical patient. Future studies of IKK function in the surgical patient, as well as translating potential therapies to modulate the IKK pathway, could ameliorate monocyte impairment and decrease the susceptibility to infection in the surgical patient. 


\section{TABLE OF CONTENTS}

ACKNOWLEDGEMENTS iii

ABSTRACT V v

LIST OF FIGURES ix

LIST OF TABLES xiii

I. GENERAL INTRODUCTION 1

II. THE SIGNIFICANCE OF MONOCYTE IMPAIRMENT IN THE SURGICAL PATIENT

a. Background 5

b. The role of the healthy monocyte 12

C. Clinical scenarios and the monocyte response 16

d. Patient factors influencing monocyte function 23

e. Defining and studying the impaired monocyte 26

f. Inhibitor of Kappa B Kinase (IKK) 31

III. PAST, PRESENT AND FUTURE OF AUGMENTATION OF MONOCYTE FUNCTION IN THE SURGICAL PATIENT 37

$\begin{array}{ll}\text { a. Background } & 37\end{array}$

b. Clinical evidence for therapies (IFN-y, GM-CSF \& G-CSF) 42

C. Challenges of therapy 51

d. The future of monocyte biology in the surgical patient 55

e. Future immunoadjuvants 56

f. Conclusion 58

IV. HYPOTHESIS AND EXPERIMENTAL PLAN 60

$\begin{array}{ll}\text { a. Purpose } & 60\end{array}$

b. Hypothesis 60

C. Specific Aims 60

d. Experimental plan 61

e. Methods 63

i. Monocyte isolation 63

ii. In-vitro induced monocyte impairment 64

iii. Cytokine assays $\quad 65$ 
iv. Flow cytometry 65

v. RNA isolation 66

$\begin{array}{ll}\text { vi. mRNA measurements } & 67\end{array}$

vii. miRNA measurements 68

viii. Western Blot analyses 68

ix. IKK inhibition 70

x. Statistical Analyses 71

V. DEVELOPING A MODEL OF MONOCYTE IMPAIRMENT 72

a. Introduction 72

$\begin{array}{ll}\text { b. Results } & 73\end{array}$

C. Discussion 88

VI. IKK SIGNALING IN THE IMPAIRED MONOCYTE 93

a. Introduction 93

b. Results 94

C. Discussion 120

VII. THE ROLE OF IKK IN THE INFLAMMATORY RESPONSE 128

a. Introduction 128

b. Results 134

C. Discussion 147

VIII. EFFECTS OF IMMUNE ADJUVANTS ON THE IMPAIRED MONOCYTE 165

a. Introduction 165

b. Results 170

C. Discussion 179

IX. MONOCYTE FUNCTION IN THE INFECTED SURGICAL PATIENT

a. Introduction 182

b. Results 186

C. Discussion 193

$\begin{array}{lll}\text { X. CONCLUSIONS AND OVERVIEW } & 198\end{array}$

$\begin{array}{ll}\text { REFERENCES } & 203\end{array}$

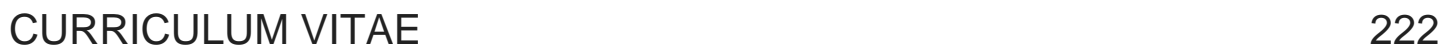




\section{LIST OF FIGURES}

1. The immune response in the critically-ill surgical patient. 3

2. Monocyte HLA-DR expression and outcomes in trauma patients. 8

3. Muramyl dipeptide (MDP) augmentation of bacterial $\begin{array}{ll}\text { containment in a model of wound contamination } & 10\end{array}$

4. Basic monocyte functions. 13

5. Negative regulators involved in monocyte impairment (endotoxin $\begin{array}{ll}\text { tolerance). } & 28\end{array}$

6. The role of the Inhibitor of KB Kinase (IKK) pathway in the activation of NFKB

7. Principle of in-vitro studies of the impaired monocyte. 40

8. Human in-vivo monocyte impairment.

9. Effect of IFN-y on monocyte HLA-DR expression in severely traumatized patients.

10. Therapeutic efficacy of mediator-targeted therapy for sepsis.

11. Hypothesis.

12. Monocyte purity following CD14 magnetic bead positive selection from whole blood.

13. Time course of monocyte viability in culture.

14. Experimental design of the impaired monocyte model.

15. TNF- $\alpha$ production in the impaired monocyte.

16. IL-10 production in the impaired monocyte. 
17. HLA-DR expression in the impaired monocyte.

18. IL-6 production in the impaired monocyte.

19. TNF- $\alpha$ gene expression (mRNA) in the impaired monocyte.

20. Factors influencing the probability of infection in the surgical patient.92

21. NFKB (p65) levels in the impaired monocyte.

22. IKB signaling in the impaired monocyte.

23. IKK mRNA expression in the impaired monocyte.

100

24. IKK- $\alpha$ protein expression in the impaired monocyte.

25. IKK- $\beta$ protein expression in the impaired monocyte. 102

26. IKK phosphorylation in the impaired monocyte.

27. TAK-1 signaling in the impaired monocyte.

28. Toll-like receptor signaling in the impaired monocyte. 106

29. Effect of IKK-16 on IKK phosphorylation. 109

30. Effect of IKK-16 on monocyte viability.

31. Effect of IKK-16 on TNF- $\alpha$ production.

32. Effect of IKK-16 on IL-10 production.

33. Effect of IKK-16 on HLA-DR expression.

34. miRNA screening of the impaired monocyte.

35. Verification of miRNA dysregulation in the impaired monocyte.

36. Effect of IKK-16 on miRNA-155 expression.

37. Power and sample size graph for IL-10 at 12 hours following $100 \mathrm{ng} / \mathrm{mL}$ LPS challenge

38. Experimental design of Multiplex experiments.

39. Effects of monocyte impairment and IKK inhibition on pro-inflammatory cytokine production. 
40. Effect of monocyte impairment and IKK inhibition on anti-inflammatory cytokine production.

41. Effects of monocyte impairment and IKK inhibition on chemokine production.

42. Effect of monocyte impairment and IKK inhibition on growth factor production.

43. Effect of monocyte impairment and IKK inhibition on the production of other immunoregulatory cytokines.

44. Relative change of cytokine and chemokine production in the impaired monocyte compared to naïve monocytes.

45. Summary of the effect of monocyte impairment and IKK inhibition on cytokine and chemokine networks (Venn diagram).

46. The genomic response to trauma and potential role of $\mathrm{IKK}$ inhibition.

47. Pathway analysis of the impaired monocyte.

48. KEGG pathway of Toll-like Receptor Signaling.

49. Experimental design of adjuvant therapy experiments.

50. Effect of adjuvants on TNF-a production.

51. Effects of adjuvants on IL-10 production.

52. Effects of adjuvants on HLA-DR expression.

53. Effects of adjuvants on CD14 expression.

54. Effects of adjuvants on PD-L1 expression.

55. Effects of adjuvant treatments on cell viability.

56. Experimental design of clinical study of patients with surgical infection. 
57. Monocyte HLA-DR expression in patients with surgical infection. 188

58. Plasma TNF- $\alpha$ concentration in patients with surgical infection. 189

59. Plasma IL-10 concentration in patients with surgical infection. 190

60. Plasma IL-6 concentration in patients with surgical infection. 191

61. Monocyte IRAK-M expression in patients with surgical infection. 192

62. Proposed mechanism of IRAK-M mediated suppression of monocyte function in patients with surgical infection. 196

63. Summary of dissertation findings. 201 


\section{LIST OF TABLES}

1. List of abbreviations.

2. Salient observations from selected papers relating monocyte $\begin{array}{ll}\text { function to clinical outcome. } & 18\end{array}$

3. Clinical factors influencing monocyte function. 25

4. Current clinical trials relating to monocyte function. 57

5. Power and sample size calculations for Multiplex cytokine/chemokine analysis.

6. Effect of monocyte impairment and IKK inhibition on cytokine/chemokine production.

7. Top 5 networks involved in monocyte impairment based on pathway analysis (Metacore ${ }^{\mathrm{TM}}$ ) 


\section{CHAPTER I}

\section{GENERAL INTRODUCTION}

Major insults to the body, such as trauma, surgery or infection, can lead to a complex and sometimes harmful inflammatory response. The systemic release of DAMPs and PAMPs stimulate toll-like receptors to cause an initial upregulation of pro-inflammatory cytokines which is met with a downregulation of antigen presenting proteins ${ }^{1}$. A loss of this fine balance can result in impaired monocyte function which is typified by decreased cytokine production (e.g. tumor-necrosis factor alpha (TNF- $\alpha$ )) and decreased HLA-DR expression ${ }^{2,3}$. Monocyte impairment, which is seen in approximately a sixth of patients after such an insult, has repeatedly predicted infective complications and mortality ${ }^{4}$.

The pathophysiological response underpinning this form of immunosuppression is complex and poorly understood (Figure 1). The commonly held belief is that as a result of the initial stimulation, such as from lipopolysaccharide (LPS), negative feedback molecules increase which inhibits toll-like receptor signaling, thus preventing the propagation of any potential responses from subsequent stimuli ${ }^{5}$. Numerous observations from clinical studies and from animal and in-vitro models suggest that important intracellular inflammatory signaling pathways such as nuclear factor kappa-B (NFKB) are subsequently suppressed, resulting in a decreased cytokine response ${ }^{6,7}$. 
Due to such an incomplete understanding of how host defenses respond to an endotoxin challenge, there are no early, reliable validated markers for identifying and predicting patients with monocyte impairment who are at risk of complications at the time of writing ${ }^{8}$. Furthermore, there are no clinically proven adjunctive immune therapies to improve mortality by augmentation of monocyte function in this subset of surgical patients ${ }^{9}$. The purpose of this work is to develop a model of impaired monocyte function in order to understand the changes in inflammatory signaling pathways that result in a suppressed monocyte inflammatory response, and specifically the role and regulation of Inhibitor of Kappa-B Kinase (IKK), a critical link between toll-like receptor signaling and NFKB activation in this setting. 


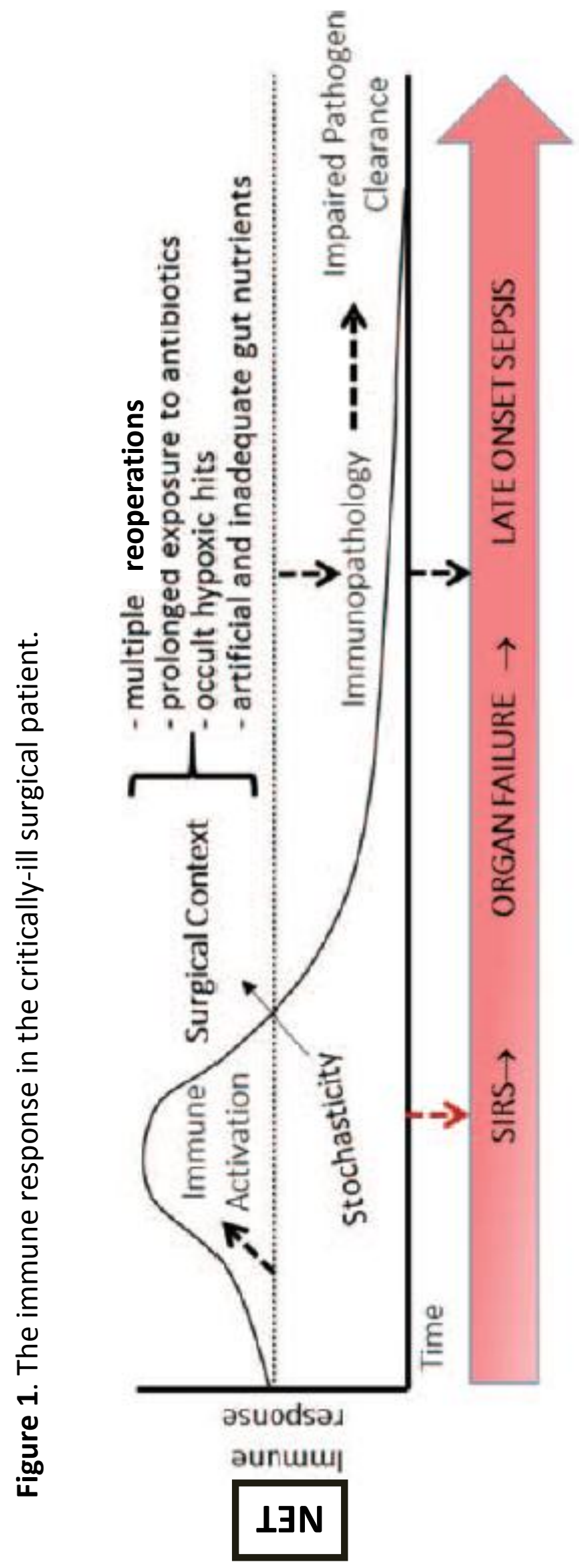




\section{Legend to Figure 1.}

Surgical insults such as trauma, major infection and surgery itself leads to poorly understood pro-inflammatory and compensatory anti-inflammatory mechanisms. Approximately a sixth of patients will develop impaired monocyte function. This immunopathology is a contributory factor to the death of some surgical patients, and remains a potentially modifiable aspect of surgical care. Challenges remain in defining which patient, which adjuvant to give, when and for how long to deliver it. A better understanding of this complex immune response could help answer these questions.

(Adapted from Krezalek et al, Shock, $2016^{10}$ ). 


\section{CHAPTER II \\ THE SIGNIFICANCE OF MONOCYTE IMPAIRMENT IN THE SURGICAL PATIENT}

\section{a. Background}

Significant insults to the body such as trauma, major surgery and overt sepsis of other origins are associated with the release of danger associated molecular patterns and/or pathogen associated molecular patterns. In the surgical patient, these can range from gram negative bacterial of enteric origin to intracellular proteins and mitochondrial DNA from cell necrosis as a result of major trauma. These molecules act as agonists to numerous receptors, such as toll-like receptors, expressed on the surface of circulating and fixed leukocytes. Systemic release of signaling molecules, including cytokines and acute phase proteins lead the systemic inflammatory response with a subsequent cascade of host defense dysregulation. If this phase is excessive or prolonged, it is thought by some to lead to harmful consequences, including multi-organ failure and death. The traditionally taught tri-phasic model of death following trauma describes many patients who survive the initial insult then suffer later infections in a setting of immune suppression, engendering the term "immunoparalysis" ${ }^{11-14}$. This chapter aims to give an overview of what we believe to be a central component of this process: monocyte impairment in the surgical patient. In this chapter, the work of often large 
groups is identified by the first and/or senior author of the reference. Table 1 is a list of abbreviations. 
Table 1. List of abbreviations.

\begin{tabular}{|c|c|c|c|}
\hline A20 & See TNFAIP & MKP1 & MAPK phosphatase-1 \\
\hline BCL & B-cell CLL lymphoma & MyD88 & $\begin{array}{l}\text { Myeloid differentiation primary } \\
\text { response gene } 88\end{array}$ \\
\hline CaCP29 & $\begin{array}{l}\text { Monoclonal antibody targeting the } \\
\text { complement cascade }\end{array}$ & NEMO & NFKB essential modulator \\
\hline CCL & Chemokine ( $\mathrm{C}-\mathrm{C}$ motif) ligand & NFKB & $\begin{array}{l}\text { Nuclear factor kappa-light-chain } \\
\text { enhancer of activated B cells }\end{array}$ \\
\hline $\begin{array}{l}\text { CD } \\
(14 / 16)\end{array}$ & Cluster differentiation & NK cells & Natural killer cells \\
\hline CLP & Cecal ligation and puncture & NOD & $\begin{array}{l}\text { Nucleotide-binding oligomerization } \\
\text { domain }\end{array}$ \\
\hline CREB & $\begin{array}{l}\text { cyclic AMP responsive element binding } \\
\text { protein }\end{array}$ & P65 & A subunit of the NFKB complex \\
\hline CXCL & Chemokine (C-X-C motif) ligand & PAMP & $\begin{array}{l}\text { Pathogen associated molecular } \\
\text { patterns }\end{array}$ \\
\hline DAMP & Danger associated molecular patterns & PBMC & Peripheral blood mononuclear cells \\
\hline ELISA & Enzyme linked immunosorbent assay & PD-1 & Programmed cell death 1 \\
\hline G-CSF & Granulocyte colony stimulating factor & PD-L1 & Programmed cell death ligand 1 \\
\hline GAPDH & $\begin{array}{l}\text { Glyceraldehyde 3-phosphate } \\
\text { dehydrogenase }\end{array}$ & & \\
\hline GM-CSF & Granulocyte-macrophage stimulating factor & Poly (I:C) & Polyinosinic:polycytidylic acid \\
\hline GUSB & Glucuronidase Beta & & \\
\hline HLA-DR & Human leukocyte antigen DR & PPAR & $\begin{array}{l}\text { Peroxisome proliferation-activated } \\
\text { receptor }\end{array}$ \\
\hline HMGB1 & High mobility group box 1 & & \\
\hline HRPT & Hypoxanthine Phosphoribosyltransferase & RAGE & $\begin{array}{l}\text { receptor for advanced glycation end- } \\
\text { products }\end{array}$ \\
\hline ICU & Intensive care unit & RAW 264.7 & $\begin{array}{l}\text { Name of a commonly used murine } \\
\text { macrophage cell line }\end{array}$ \\
\hline IFN & Interferon & RCT & Randomized controlled trial \\
\hline $\begin{array}{l}\text { IL }(1,6, \\
10,12)\end{array}$ & Interleukin & RNA & Ribonucleic acid \\
\hline IRAK & IL-1 $\beta$ receptor associated kinase & ROI/ROS & $\begin{array}{l}\text { Reactive oxygen } \\
\text { intermediates/species }\end{array}$ \\
\hline IRF & Interferon regulatory factor & socs & Suppressor of cytokine signaling \\
\hline IKB & Inhibitor of $\mathrm{KB}$ & STAT & $\begin{array}{l}\text { Signal transducer and activator of } \\
\text { transcription }\end{array}$ \\
\hline IKK & Inhibitor of $\mathrm{kB}$ kinase & TAK1 & $\begin{array}{l}\text { Transforming growth factor beta- } \\
\text { activating kinase } 1\end{array}$ \\
\hline JAK & Janus kinase & TCR & T-cell receptor \\
\hline LPS & Lipopolysaccharide & $\mathrm{TH}(1 / 2)$ & T-helper \\
\hline LTA & Lipoteichoic acid & $\begin{array}{l}\text { THP1 \& } \\
\text { U937 }\end{array}$ & $\begin{array}{l}\text { Names of commonly used human } \\
\text { monocytic cell lines }\end{array}$ \\
\hline MAPK & Mitogen-activated protein kinase & THR & Total hip replacement \\
\hline MCP-1 & Monocyte chemotactic protein-1 & TLR & Toll-like receptor \\
\hline MD-2 & Lymphocyte antigen 96 & TNFAIP & $\begin{array}{l}\text { Tumor necrosis factor alpha inducible } \\
\text { protein }\end{array}$ \\
\hline MDP & Muramyl dipeptide & TNF- $\alpha$ & Tumor necrosis factor alpha \\
\hline MHC & Major histocompatibility complex & TRAF6 & $\begin{array}{l}\text { Tumor necrosis factor receptor } \\
\text { associated factor }\end{array}$ \\
\hline MIG & Monokine inducible by interferon- $\gamma$ & TRIF & $\begin{array}{l}\text { Toll/IL-1 receptor domain-containing } \\
\text { adaptor-inducing interferon- } \beta\end{array}$ \\
\hline
\end{tabular}


Figure 2. Monocyte HLA-DR expression and outcomes in trauma patients.

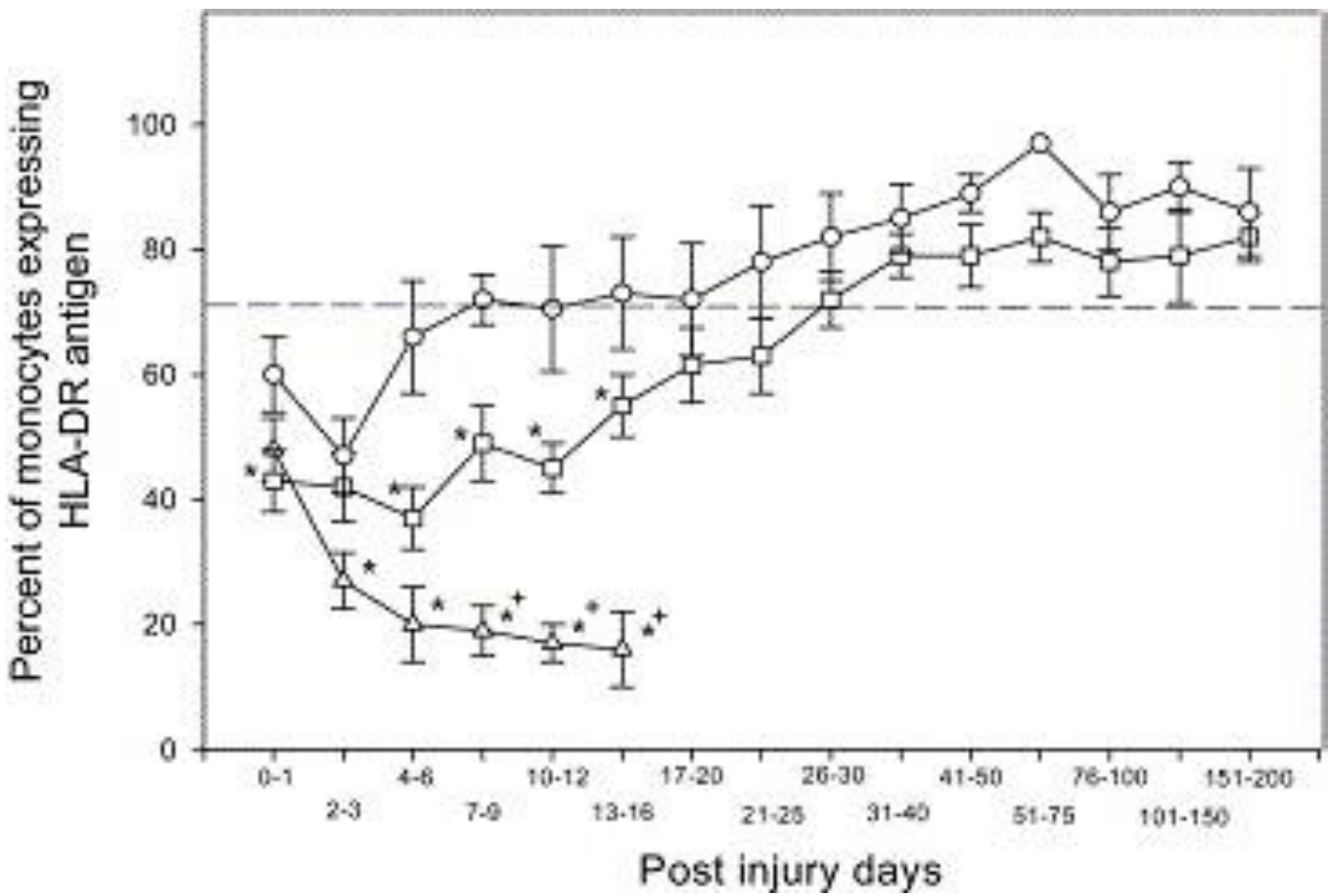

Outcomes are labeled as an uncomplicated recovery $(O)$, survivors with posttraumatic infection ( $\square$ ) and non-survivors $(\Delta)$. Note the patient trajectory between groups becomes apparent only by day 4-6 after injury, based on this marker.

(From Hershman et al, Br J Surg, 1990. ${ }^{15}$ ) 
Systematic observations of host defense mechanisms in ill patients prompted the recognition of the importance of the impaired monocyte, contrary to their null hypothesis ${ }^{4}$. Specifically, a subset of approximately $15 \%$ of patients exhibited subnormal monocyte function and were at higher risk of developing infection and of death ${ }^{15}$. This is illustrated in Figure 2, where differences in monocyte HLA-DR expression, a cell surface marker, become increasingly predictive of a patients outcome after days 4-6 following major trauma.

The ultimate goal for laboratory and clinical investigators is not just to identify such "high risk" patients, but also to augment host defenses. This principle is illustrated in Figure 3, where the addition of muramyl dipeptide (MDP) in a rat model of a contaminated wound promotes containment, as opposed to permissive progression to bacteremia and subsequent systemic deleterious consequences ${ }^{16}$.

The central factor that determines a patient's fate following a major insult remains unclear. In a group of patients given the same equivalent insult, many will be "aces" and survive, but a subset will be "dogs" and not survive (Fry DE, unpublished). Firstly, genetic predisposition can lead to subnormal host defenses. Secondly, the heterogeneous nature of an insult and its severity can also be decisive and thirdly, epigenetic changes, such as in microRNA (miRNA), may significantly result in post-translational modification of important pro- and anti-inflammatory signaling. Finally, prompt and attentive supportive care with timely surgical intervention and precise non-operative care can massively shift the balance for some patients. 


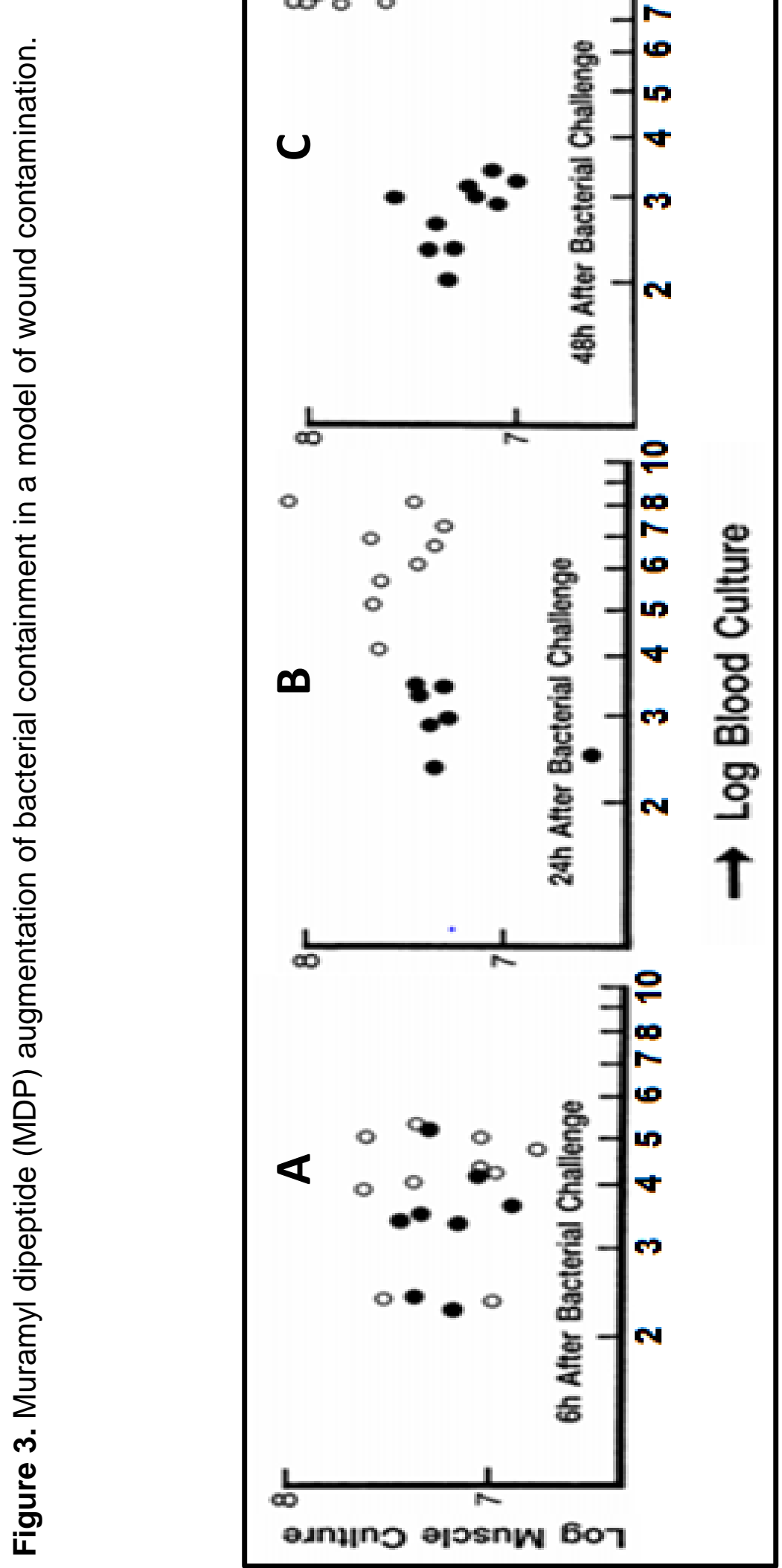




\section{Legend to Figure 3.}

Infection in skeletal muscle is contained better by MDP (•) than by placebo (O). As time passes from 6 hours after contamination in A, note MDP produces constant levels of localized bacteria where placebo treated animals experienced escape of bacteria into blood by 48 hours $(C)$. This experiment exemplifies the potential clinical benefit from immune enhancement. (Adapted from Polk et al, Infect Immun, 1990. ${ }^{16}$ ) 


\section{b. The role of the healthy monocyte}

The monocyte is central to the innate immune system in the surgical patient. Its key roles are cytokine production, antigen presentation, and phagocytosis (Figure 4). Early recognition of PAMPs \& DAMPs from invading microbes and damaged tissue activate monocytes through surface receptors such as extracellular toll like receptors, and intracellular receptors such as NOD-like receptors, RAGE receptors and certain toll-like receptors.

This leads to the infiltration of neutrophils, traditionally the first leukocyte on the scene. These polymorphonuclear cells have the most potent phagocytic ability, which are followed by infiltrating circulating monocytes due to chemokines. These can differentiate and thus expand the numbers of tissue macrophages. Throughout this process, monocytes secrete cytokines which have systemic consequences on remote organs such as the liver, gastrointestinal system and endothelium, especially that in lung parenchyma. Furthermore, this cell-to-cell communication leads to a crucial link to the adaptive immune system through complex T-cell interactions. The monocyte, and other antigen presenting cells, allow fragments of invading bacteria, such as LPS, to be processed in vesicles and externalized through MHC II complexes, such as HLA-DR, that bind to T-cell receptors on receptive Tcells. This bi-directional interaction can also further promote monocyte/macrophage activation through endogenous IFN- $y$ from T-cells and natural killer cells. However, in patients with a subnormal immune system or persistent antigen stimulation, this process can be blunted through the promotion of negative co-stimulatory molecules such as PD-1/PD-L1 surface molecules. 
Figure 4. Basic monocyte functions.

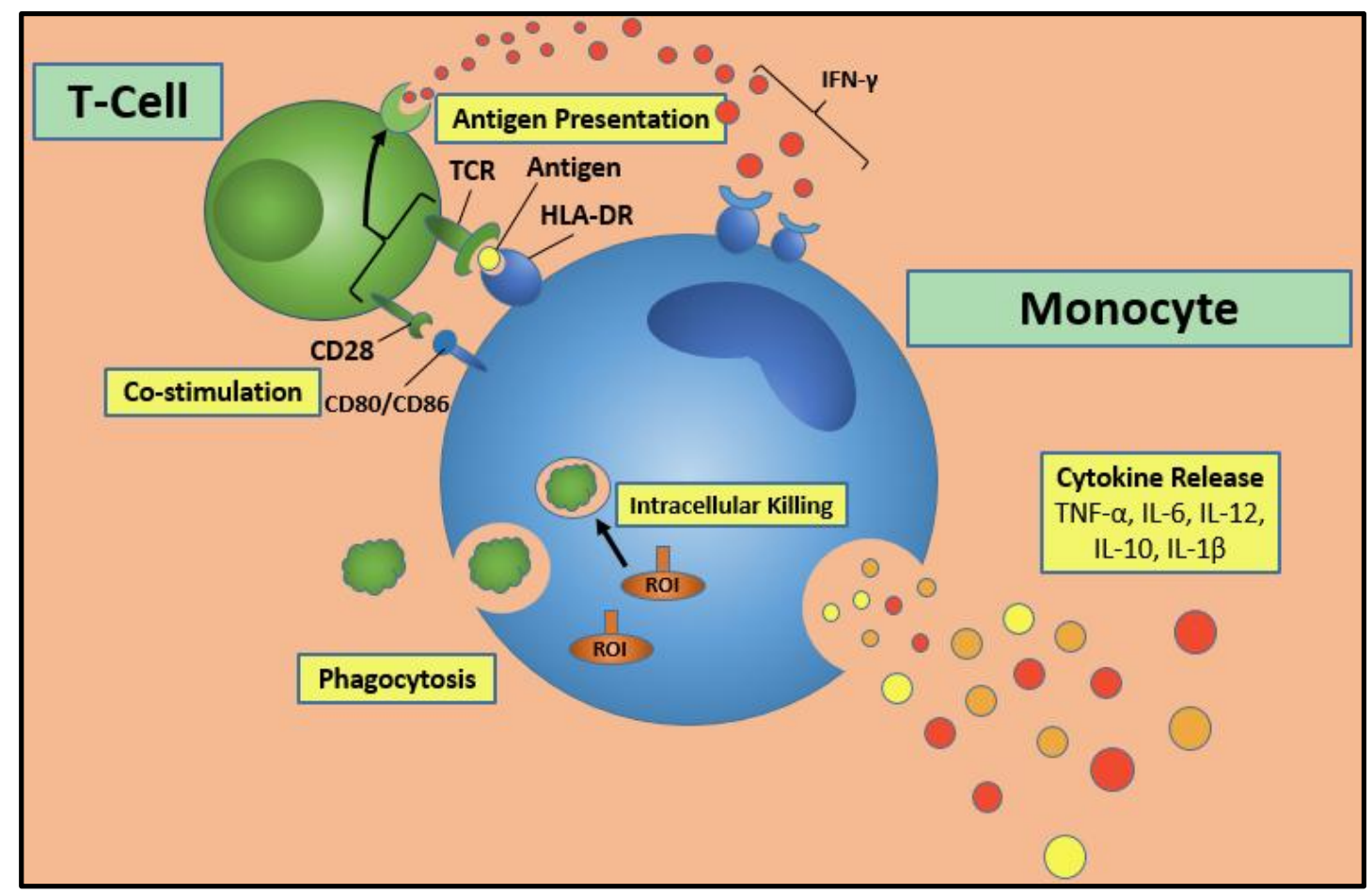

The monocyte is a highly important circulating leukocyte which links the innate to adaptive immune systems. Its primary functions include cytokine production (such as TNF- $\alpha, I L-10, I L-1 \beta$ and IL-12), antigen presentation (through major histocompatibility complexes such as HLA-DR) and phagocytosis. Impairment of the first two functions have been correlated with poor outcomes in surgical patients, thus we believe that they are the two most clinically relevant functions to study.

Original figure by Norman Galbraith and Samuel Walker (student). 
Invading microbes initially evoke a T-helper (Th) 1 response from CD4 T-cells. In this type of response, T-cells secrete IFN-y and IL-2, further promoting and activating monocytes and macrophages in a pro-inflammatory manner. While this response is linked to the "M1" pro-inflammatory macrophage, the alternative Th2 response (associated with allergens and Helminths) secretes IL-10 and IL-4 promoting the "M2" anti-inflammatory macrophage.

Cytokines are secreted proteins, acting in a paracrine manner to influence the differentiation and response of surrounding immune cells. Of a multitude of cytokines that monocytes secrete, the most clinically studied (and their function) include;

- Pro-inflammatory cytokines

- TNF- $\alpha$ - promotes inflammation and endothelial activation

- IL-6 - acute phase response promoting fever and T/B cell growth

- IL-1 $\beta$ - fever, activation of monocytes, macrophages, T-cell activation

- Anti-inflammatory cytokines

- IL-10 - suppresses monocyte function

Cytokines influencing monocyte behavior are discussed below. While monocytes do phagocytize bacterial pathogens, defects in its phagocytic ability appearing to be less important in the fate of the surgical patient when compared to altered cytokine release and antigen presentation.

Monocytes are now considered to be of three main types ${ }^{17}$ : 
1. The classical monocyte accounts for the vast majority of circulating monocytes, expressing high CD14, medium HLA-DR expression, but no CD16 expression (CD14++ CD16-). These cells produce both TNF- $\alpha$ and IL-10.

2. Non-classical monocytes express lower levels of CD14, but high CD16 and HLA-DR (CD14+CD16++). They secrete TNF- $\alpha$ but not $\mathrm{IL}-10$.

3. Finally intermediate monocytes express high levels of CD14, but low CD16. (CD14++ CD16+). This subset has gained increasing recognition over the last 5 years and is noted to have increased IL10 production. Interestingly, they have been correlated with sepsis, myocardial infarction and early, but not metastatic colorectal cancer 18

It is worth noting that monocytes, macrophages and dendritic cells are all myeloid derived with largely similar expression of toll-like receptors. While this body of work focuses on the monocyte, many of the concepts discussed regarding physiology, pathology and therapy can be extended to all the above. 


\section{c. Clinical scenarios and the monocyte response}

\section{$\underline{\text { Major trauma \& burns }}$}

As post-traumatic and indeed all critical care improved, it became apparent that some survivors of major trauma were susceptible to later infection. Evidence grew that this may be a consequence of depressed host defenses 19. Our systematic study of various facets of the innate immune system revealed that monocyte antigen presenting ability was highly predictive of infection following major trauma ${ }^{4}$. It was apparent that not only absolute monocyte HLA-DR expression, but the ability to up-regulate this MHC II surface marker in response to endotoxin exposure was also predictive of morbidity and mortality. Since then, studies focusing on HLA-DR expression as a marker of monocyte function demonstrate that this remains consistently predictive for poor outcomes following trauma as well as folowing burns (Table $2^{2,4,15,20-33}$ ). Increasingly, CD14+ HLA-DR- cells are described as "myeloid derived suppressor cells" and this phenotype has also been observed in the orthopedic patient following intramedullary nailing following fracture of the tibia ${ }^{34}$.

The revolution of cytokine related research prompted another aspect of monocyte function to be examined - ex-vivo cytokine production in response to endotoxin exposure, both in "whole blood" and in isolated peripheral blood mononuclear cells. In general, early high IL-6 production and subsequent lower TNF- $\alpha$ production appears to characterize this dysregulation ${ }^{35}$. Furthermore, the role of the anti-inflammatory IL-10 has long been debated, but it remains clear that IL-10 acts to inhibit pro-inflammatory pathways. While 
a homeostatic balance is critical, IL-10 can promote a suppressed monocyte phenotype ${ }^{36}$. 
Table 2. Salient observations from selected papers relating monocyte function to clinical outcome.

\begin{tabular}{|c|c|c|c|c|}
\hline $\begin{array}{c}\text { SENIOR } \\
\text { AUTHOR }\end{array}$ & $\begin{array}{c}\text { KEY } \\
\text { OBSERVATION }\end{array}$ & CLINICAL SETTING & OUTCOME & JOURNAL, YEAR \\
\hline MONNERET ${ }^{20}$ & HLA-DR & Septic shock & Mortality & $\begin{array}{c}\text { Intens. Care M. } \\
2006\end{array}$ \\
\hline VENET $^{21}$ & HLA-DR & Burns & Septic shock & Crit Care, 2007 \\
\hline LAHIRI $^{22}$ & TLR4/5 & $\begin{array}{c}\text { Elective } \\
\text { hepatopancreatobiliary } \\
\text { surgery }\end{array}$ & $\begin{array}{l}\text { Systemic } \\
\text { inflammatory } \\
\text { response } \\
\text { syndrome }\end{array}$ & Ann. Surg, 2015 \\
\hline POLK $^{4}$ & HLA-DR & Trauma & Mortality & Ann. Surg, 1986 \\
\hline CHEADLE $^{23}$ & HLA-DR & Trauma & Infection & Am. J. Surg, 1991 \\
\hline PLODER $^{2}$ & TNF- $\alpha$ & Trauma & $\begin{array}{l}\text { Sepsis \& multi- } \\
\text { organ failure }\end{array}$ & Shock 2006 \\
\hline HERSHMAN ${ }^{15}$ & $\begin{array}{l}\text { HLA-DR } \\
\text { LPS induced } \\
\text { HLA-DR }\end{array}$ & Trauma & $\begin{array}{l}\text { Infection } \\
\text { Mortality }\end{array}$ & $\begin{array}{l}\text { British Journal of } \\
\text { Surgery, } 1990\end{array}$ \\
\hline LIN $^{24}$ & HLA-DR & Pancreatitis & $\begin{array}{l}\text { Secondary } \\
\text { infection }\end{array}$ & $\begin{array}{l}\text { Hepatogastro- } \\
\text { enterology } 2013\end{array}$ \\
\hline GAUDILLIERE ${ }^{25}$ & $\begin{array}{c}\text { STAT3, CREB, } \\
\text { NFKB }\end{array}$ & Elective THR & $\begin{array}{c}\text { Recovery, } \\
\text { function, pain }\end{array}$ & $\begin{array}{l}\text { Sci. Trans. Med. } \\
2014\end{array}$ \\
\hline LONGBOTTOM $^{26}$ & IL-6 & $\begin{array}{c}\text { Elective } \\
\text { gastrointestinal } \\
\text { surgery }\end{array}$ & $\begin{array}{l}\text { Post-operative } \\
\text { infection }\end{array}$ & Ann. Surg. 2015 \\
\hline LYONS $^{27}$ & IL-10 & Trauma & Septic events & Ann. Surg. 1997 \\
\hline STENSBALLE ${ }^{28}$ & IL-6, IL-10 & Trauma & Mortality & $\begin{array}{l}\text { Acta Anaesth. } \\
\text { Scand. } 2009\end{array}$ \\
\hline HOFFMANN ${ }^{29}$ & HLA-DR & $\begin{array}{l}\text { Pediatric lung } \\
\text { transplantation }\end{array}$ & $\begin{array}{c}\text { Infection } \\
\text { (pneumonia) }\end{array}$ & $\begin{array}{c}\text { Transp. Infect. Dis. } \\
2004\end{array}$ \\
\hline HANDY ${ }^{30}$ & $\begin{array}{l}\text { HLA-DR } \\
\text { Monocyte } \\
\text { subsets }\end{array}$ & $\begin{array}{c}\text { Elective } \\
\text { low/intermediate risk } \\
\text { surgery }\end{array}$ & $\mathrm{N} / \mathrm{A}$ & $\begin{array}{l}\text { Anesthesiology, } \\
2010\end{array}$ \\
\hline BIFFL ${ }^{\star \star 31}$ & IL-6 & Trauma & *review & Ann. Surg. 1996 \\
\hline CHERON $^{32}$ & IL-6, HLA-DR & Trauma & Sepsis & PloS One, 2012 \\
\hline GUIGNANT $^{33}$ & PD-L1 & Septic shock & $\begin{array}{l}\text { Secondary } \\
\text { infection } \\
\text { Mortality }\end{array}$ & Crit. Care. 2011 \\
\hline
\end{tabular}

${ }^{* *}$ Review article. 
Findings of the Inflammation and the Host Response to Injury Large Scale Collaborative Research Program relating to monocyte function

A highly funded multi-center project, the "Glue Grant", aimed to take a 'systems approach' to the host inflammatory response to trauma and burns, primarily focusing on gene expression studies ${ }^{1}$. This design was to lessen the reductionist approach of multiple groups focusing on one single aspect of the host defenses which was seen as likely to fail given the redundancy of the immune system. It is worth noting that the patients studied were critically ill; generally being hypotensive, having received blood products, and demonstrating some form of organ dysfunction. Selected papers from this effort pertaining to monocyte impairment are discussed below.

Xaio et al ${ }^{1}$ sequentially studied peripheral blood leukocyte genome wide expression of mRNA in 167 severely injured trauma patients up to 28 days after injury. In addition, patients with greater than $20 \%$ burns and healthy individuals were analyzed. A change in over $80 \%$ of gene expression, termed a "genomic storm", was observed. This response to trauma was non-specific, with $98 \%$ of the same genomic changes seen in burn patients, and $88 \%$ of that seen in experimental endotoxemia. A complicated recovery was associated with the up-regulation of innate immunity genes including IL-6 and IL-10, with a simultaneous down-regulation of adaptive immunity genes such as those relating to antigen presentation. Importantly, they demonstrated that contrary to conventional thinking, leukocyte genomic responses of both proand anti-inflammatory pathways were simultaneous, rather than a so-called

\footnotetext{
${ }^{1}$ The Glue Grant: Inflammatory and the host response to injury (PI: Tompkins RG). National Institute of General Medical Sciences.
} 
"second hit" or insult causing the anti-inflammatory component ${ }^{1}$. Laudanski et al examined both monocyte and T-cell pathways of 18 trauma patients using high throughput screening of gene expression ${ }^{37}$. Interestingly, the response to trauma was very specific to the cell type, with only $11 \%$ of pathways common to both monocytes and T-cells, such as a down-regulation of the transcription factor NFkB. This further demonstrated a down-regulation of antigen presentation genes such as HLA-DR and CD86, with an increase in monocyte negative co-stimulatory genes such as PD-L2. In keeping with the down-regulation of antigen presenting pathways in monocytes, T-cells demonstrated molecular choreography with reciprocal increases in genes relating to apoptosis and anergy ${ }^{37}$.

In a more recent study from the same dataset, $8 \%$ of the 1595 subjects included had proven gram negative bacteremia. These 127 patients had an increased risk of mortality (21\%), compared with those without infection (11\%). Gene expression of these patients was compared with those who did not contract infection, showing certain pro-inflammatory pathways to be similar 12 hours following injury. However, at 96 hours after injury IL-1 $\beta$ and IL-2RB were markedly decreased in those who died. Furthermore, expression of genes relating to monocytes such as HLA-DR and CD163 was similarly increased at 12 hours after injury, but at 96 hours a significant decrease in gene expression were apparent in the group who went on to develop gram negative bacteremia ${ }^{38}$. This project has increased our understanding of the pathways involved in host defenses following major trauma. Perhaps not surprisingly given the recognition of such complexity, the surgeon remains illequipped to confidently or specifically modulate this response. 
Inflammatory networks following trauma

Since the prior project came to an end, other groups have adopted a 'systems biology' approach to better understand inflammatory networks after major trauma. The Pittsburgh group studied 472 survivors of blunt trauma for circulating plasma cytokines and chemokines. The findings were analyzed using emerging computational methods reflecting that various patterns were demonstrable in the first 24 hours, much earlier than the diagnosis of infection made 7 days after injury. Serum markers that were significantly elevated in patients who later developed infection include HMGB1, IL-6 and IL-1RA, to name a few. Dynamic Bayesian networks inferred that IL-6 was affected by various chemokines, such as MCP-1 and MIG ${ }^{39,40}$.

\section{Major elective surgery}

Major abdominal operations, and general anesthesia, have long been associated with post-operative immunosuppression. Factors such as increasing age, prevalence of diabetes mellitus and immunosuppressive medications (neoadjuvant chemotherapy, glucocorticoids and monoclonal antibodies such as anti-TNF- $\alpha$ antibodies) increase the relevance of this concept today. However, the prevalence of impaired monocyte function also extends to the patients of neurosurgeons, transplant surgeons and cardiac surgeons ${ }^{41-43}$. More recently, Albertsmeier showed that major abdominal surgery leads to an expansion of CD14+ HLA-DR- cells. This group of monocytes secreted TNF- $\alpha$ and IL-6, but when co-cultured with T-cells led to decreased T-cell IFN-y secretion ${ }^{44}$. Studies in the last few years have demonstrated how surgical approaches can also influence monocyte 
behavior, as discussed later. Furthermore, dysregulation of pro-inflammatory pathways in monocyte subsets, such as NFKB in intermediate monocytes, led to higher TLR and IL-6 expression that was associated with increased infective complications ${ }^{22,26}$.

\section{$\underline{\text { Overt sepsis }}$}

Major infection, namely severe sepsis and septic shock, is the most common clinical event associated with monocyte impairment. While the majority of studies use flow cytometry to quantify either the percentage of monocytes expressing HLA-DR, or mean fluorescence intensity, more recent studies show that gene expression of HLA-DR is also predictive of infective complications $^{21,45}$. Furthermore, bench to bedside studies of monocyte impairment reveal dysregulated gene expression patterns, including genes such as S100A8/9, TNFAIP6 and IRAK-M (as well as TNF-a, IL-10 and HLADR) which have been suggested to "monitor efficacy" of IFN-y therapy ${ }^{46-48}$.

There has been increased interest over the past decade in the differential expression of negative co-stimulatory molecules in septic patients. This has been in part due to the recognition of T-cell anergy and apoptosis as a driver of immune suppression, which is also observed in some cancers ${ }^{49}$. Monocyte co-stimulatory antigens such as CD86 and PD-L1 correspond to Tcell CD28 \& PD-1 respectively, which may be considered "on" and "off" switches in addition to the central HLA-DR-T-cell receptor (TCR) interaction. The potential importance of negative co-stimulatory molecules has been highlighted by reported improved mortality in murine models of surgical sepsis from PD-1/PD-L1 blockade ${ }^{50,51}$. Various clinical observations have found 
increased monocyte PD-L1 as well as increased T-cell PD-1 expression in infected patients. In one study of 64 patients with septic shock, of which $42 \%$ had an abdominal source, had increased expression of monocyte PD-L1 and T-cell PD-1 compared to healthy controls, which was also predictive of secondary nosocomial infection ${ }^{33}$. In another study of 90 critically ill surgical patients, blood samples within 24 hours of admission to the ICU showed increased monocyte PD-1 expression, correlating with APACHE II scores ${ }^{52}$. In addition, certain PD-1 polymorphisms have been associated with higher mortality in a study of 221 patients diagnosed with sepsis or septic shock, in which lung infections were the most common source of infection ${ }^{53}$.

\section{d. Patient factors influencing monocyte function}

The practicing surgeon caring for sick surgical patients may adversely influence monocyte function unknowingly, as summarized in Table $3^{54-63}$. Experimental models have shown that protecting patients from unintentional hypothermia may promote the pro-inflammatory response through increased miRNA-155 expression and subsequent higher TNF- $\alpha$ and lower IL-10 production ${ }^{54}$. The once popular very precise glucose control in critical care environments may risk harmful hypoglycemic episodes, but also blunt the cytokine and bactericidal properties of the monocyte ${ }^{55}$. Supplemental oxygen, to an inspired oxygen concentration of $80 \%$ appears to have mixed effects on the monocyte and in particular increases reactive oxygen species ${ }^{56}$. The immunosuppressive effects of blood transfusion have been recognized for many years, but recent studies implicate small circulating biomolecules, such as protein-bound or exosomal miRNAs that may also inhibit monocyte HLA- 
DR expression ${ }^{58}$. The role of minimally invasive surgery on monocyte function has remained controversial ${ }^{64}$. Recent studies have suggested that minimally invasive surgery, such as laparoscopic or robotic approaches, are less immunosuppressive, as compared to major open colorectal and liver surgery ${ }^{59-63}$. 
Table 3. Clinical factors influencing monocyte function.

\begin{tabular}{|c|c|c|c|}
\hline Clinical factor & Parameter & Impact & Selected references \\
\hline Temperature (hyperthermia) & TNF- $\alpha$ & Increase & Billeter, FASEB, 2014 \\
\hline Glucose (hyperglycemia) & $\begin{array}{l}\text { TNF- } \alpha \\
\text { Reactive oxygen } \\
\text { species }\end{array}$ & $\begin{array}{l}\text { Increase } \\
\text { Decrease }\end{array}$ & Qadan, JACS, $2010^{55}$ \\
\hline Oxygen (supplemental) & $\begin{array}{c}\text { TNF- } \alpha \\
\text { Reactive oxygen } \\
\text { species }\end{array}$ & $\begin{array}{l}\text { Decrease } \\
\text { Increase }\end{array}$ & $\begin{array}{c}\text { Qadan, } \\
\text { Anaesthesiology, } 2010 \\
56\end{array}$ \\
\hline Blood Transfusion & $\begin{array}{l}\text { LPS induced TNF- } \alpha \\
\text { IL-6 }\end{array}$ & Decrease & $\begin{array}{c}\text { Muszynski, } \\
\text { Transfusion, } 2014 \text { \& } \\
2015^{57,58}\end{array}$ \\
\hline $\begin{array}{l}\text { Laparoscopic (over open total } \\
\text { mesorectal excision) }\end{array}$ & $\begin{array}{l}\text { HLA-DR } \\
\text { IL-6 }\end{array}$ & $\begin{array}{l}\text { Increase } \\
\text { Decrease }\end{array}$ & $\begin{array}{c}\text { Veenhof, Int. J. } \\
\text { Colorec. Dis, } 2011^{59}\end{array}$ \\
\hline $\begin{array}{l}\text { Robotic (over open } \\
\quad \text { colectomy) }\end{array}$ & $\begin{array}{l}\text { HLA-DR (equivalent } \\
\text { to laparoscopic) }\end{array}$ & Increase & $\begin{array}{l}\text { Shibata, Tech. } \\
\text { Coloproc. } 2015^{60}\end{array}$ \\
\hline $\begin{array}{l}\text { Laparoscopic (over open liver } \\
\text { resection) }\end{array}$ & HLA-DR & Increase & Chopra, JSLS, $2013^{61}$ \\
\hline $\begin{array}{l}\text { Laparoscopic (over open } \\
\text { contaminated } \\
\text { cholecystectomy) }\end{array}$ & HLA-DR & Increase & Sista, WJGS, $2013^{62}$ \\
\hline $\begin{array}{l}\text { Laparoscopic (better than } \\
\text { open colectomy, } \\
\text { independent of fast-track) }\end{array}$ & HLA-DR & Increase & $\begin{array}{c}\text { Veenhof, Ann. Surg., } \\
2012^{63}\end{array}$ \\
\hline
\end{tabular}




\section{e. Defining and studying the impaired monocyte}

Clinical studies have shown that monocyte impairment, also described as "endotoxin tolerance", is not common in most surgical patients and pertains to only some previously unsuspected patients. Therefore, it becomes difficult to adequately power and fund clinical studies to examine mechanisms behind this pathology.

Over the last 15 years, both in-vitro and in-vivo murine models have been used to study endotoxin tolerance. Endotoxin tolerance was discovered by Beeson, who found that repeated injection of endotoxin led to a progressively decreased febrile response ${ }^{65}$. We now know that exposure to endotoxin, often LPS, stimulates various negative regulators to inhibit a subsequent response when compared to a naïve cell. This has been shown to be akin to the impaired monocyte response seen in the critically ill surgical patient ${ }^{7}$. We consider monocyte impairment and endotoxin tolerance in monocytes to be a very similar phenomenon. Various studies have shown "cross-tolerance", in that the same molecular mechanisms behind such impairment also occur with other so called "priming" agents including:

- PAMPs/DAMPs

○ Lipoteichoic acid (TLR2)

- Poly (I:C) (TLR3)

- High mobility box group 1 (TLR2 \& 4)

- Cytokines

○ IL-1 $\beta$

○ IL-6 
- TNF- $\alpha$

- $\mathrm{IL}-10^{5}$

Common models include isolated primary cells such as CD14 monocytes and PBMCs as well as cell lines such as THP1 and U937 monocytes, and RAW 264.7 macrophages. In-vivo models expose mice to low dose intraperitoneal LPS, followed either by ex-vivo stimulation, or in-vivo cecal ligation and puncture. Human in-vivo models have used subcutaneous LPS, followed by ex-vivo leukocyte stimulation ${ }^{66}$. The most accepted in-vitro outcome of the LPS pre-treated "impaired" cell is reduced TNF- $\alpha$ production compared to naïve cells, measured either by supernatant protein secretion by ELISA or gene expression of TNF-a mRNA. Other cytokines have been reported variably ${ }^{5}$. Although reported less frequently, this approach is thought to represent monocytes with reduced antigen presenting capability ${ }^{67,68}$.

Pathophysiological mechanisms behind the impairment of the monocyte response are complex and influence various molecular pathways $\left(\right.$ Figure $5^{5}$ ). In toll-like receptor signaling, the MyD88-dependent pathway is inhibited at various levels such as sMyD88 and IRAK-M that inhibits TRAF6. Furthermore, A20 increases and thus inhibits TRAF6 via deubiquitination. It is at this level that Pellino-1, a positive regulator, is decreased in impaired cells 69. Recently monocyte impairment has also been associated with disruption of the interaction between IRAK1 and IKK-y (also known as NEMO) although the level and function of IKK- $\alpha$ and $-\beta$ are relatively unknown ${ }^{70}$. 


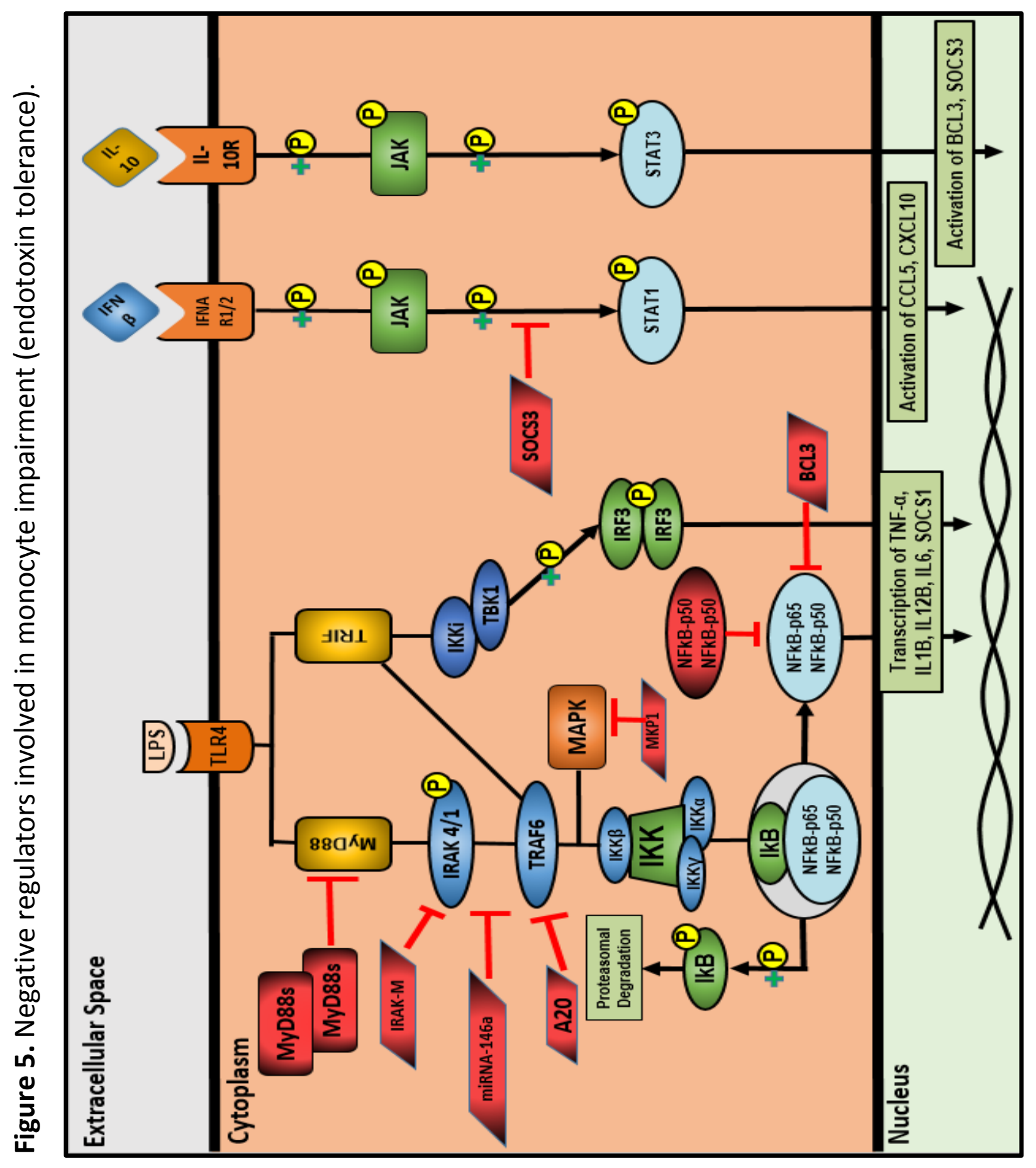




\section{Legend for Figure 5.}

Homeostatic mechanisms to prevent excessive inflammation and promote the resolution of the response are in part regulated by intracellular negative regulator molecules. These regulators act to suppress and downregulate the production and/or activation of key pro-inflammatory pathways. Note a number of epigenetic mechanisms that control toll-like receptor signaling exist, such as post-transcriptional silencing by microRNA-146a, and A20 which promotes the deubiquintation of TRAF-6.

(Modified from Biswas, Frontiers in Immunology, 2009 ${ }^{5}$.) 
The initial LPS stimulation decreases the degradation of IKB in impaired monocytes, which act as the "brakes" in the NFkB pathway. Therefore, there is decreased p65 phosphorylation and subsequent translocation to the nucleus, in part explaining the decreased gene expression of proinflammatory cytokines. Furthermore, the non-canonical NFKB pathway has been reported to be involved, with impaired cells having increased expression of RelB, c-Jun and the homodimer of p50-p50 of NFKB rather than the more commonly expressed p-65-p50 heterodimer ${ }^{71}$.

The MAPK pathway is also dysregulated, with evidence of decreased TAK1 phosphorylation in impaired cells, which leads to decreased MAPK p38 phosphorylation ${ }^{70}$. Other known negative regulators that are not discussed here include SOCS1, SOCS3, BCL3, PPAR-y and CREB ${ }^{5}$.

MicroRNAs (miRNAs) are short, non-coding RNA molecules involved in post-transcriptional regulation of gene expression. Various miRNAs are dysregulated in monocyte impairment, with miRNA-146a being the most studied. This increases as a physiological response to LPS, and subsequently inhibits TRAF6 and IRAK1. Murine knock-out or antagomiR-mediated inhibition of miRNA-146a rescues the impaired response. Further miRNAs shown to influence monocyte impairment include let-7a, miR-223, -221, -9, 150 and $-132^{5}$.

However, despite the manipulation of numerous negative regulators demonstrated in-vitro to improve the monocyte response, none of these are known to have gained scientific momentum or reached the bedside. Such complexity highlights the redundant nature of our evolved immune system. 
Furthermore, murine studies of sepsis, such as those using models of cecal ligation and puncture (CLP), have for many years raised unfulfilled hope in potential immunotherapies for the impaired monocyte, but have not yet translated to clinical success. One reason for this might be that not all of the immune system is conserved from mice to man. Murine models are overtly oversimplified - there are no additional variables such as blood transfusion, fluid resuscitation, antibiotic therapy and other co-morbidity. Finally, genetic variations in patients are obviously not represented in the narrow pool of mice and cell lines.

\section{f. Inhibitor of $\mathrm{kB}$ Kinase (IKK) - the known and unknowns governing the gateway from TLR to NFKB}

IKK is an intracellular protein complex involved in the canonical pathway of the NFkB pro-inflammatory signaling pathway. The complex is made up primarily of 3 subunits; IKK- $\alpha$, IkK- $\beta$ and IKK- $\gamma$ (also known as NEMO) (Figure 6). The first two subunits are considered catalytic subunits which are primarily involved in the propagation of a response from upstream signals, often secondary to stimulation by IL-1 $\beta$, TNF- $\alpha$ or a TLR ligand such as LPS. IKK- $\alpha$ (also known as IKK-1 or CHUK) is involved in both the canonical pathway with IKK- $\beta$, but also plays a role in the non-canonical signaling pathway in response to ligands such as lymphotoxin $B$, which activates NFKB inducing kinase (NIK) to phosphorylate IKK- $\alpha$. This in turn cleaves p52 and RelB as a heterodimer from the p100 NFkB subunit. 
IKK- $\beta$ (IKK2) is considered the main active subunit in the canonical NFKB pathway. This subunit is bound to IKK- $\alpha$, and through activation of a variety of upstream pathways (most commonly through MyD88/TRAF-6/TAK1) both the $\alpha$ and $\beta$ subunits can be phosphorylated. It should be noted that upstream input signals for IkK phosphorylation are extraordinarily complex with various levels of regulation from related kinases and phosphatases. It has been shown that IKK phosphorylation is not dependent on TAK-1 alone, with over 15 potential input sources ${ }^{72,73}$ Phosphorylation of the $\alpha \&-\beta$ subunits leads to a conformational change allowing the complex to undertake its primary role of phosphorylating $I_{k} B$, as its name suggests. I $B$ phosphorylation is the critical step in NFKB activation. IKB act as the "brakes" in this pathway, and once phosphorylation occurs IkB then undergoes ubiquitination and subsequent proteosomal degradation. This allows the main active NFKB complex comprising of the p65 (RelA) and p50 heterodimer to be "released", where it translocates from the cytoplasm to the nucleus. Here, NFkB promotes the transcription of pro-inflammatory genes such as TNF- $\alpha$, IL-10, IL-6 among many others.

The IKK-Y (NEMO) subunit is considered a regulatory unit, rather than a catalytic unit, meaning that it chaperones the $\alpha$ and $\beta$ subunits from TRAF- 6 and TAK-1 towards the NFKB subunit following activation. A fourth IKK subunit, IKK-E (Also termed IKK-i) exists but this subunit is part of a separate signaling cascade, along with TBK1, that activates the transcription factor IRF3 to promote type I interferon production. Experimental evidence suggests that IKK- $\beta$ and IKK- $\gamma$ are critical for NFKB activation in order to mount a sufficient pro-inflammatory response against stimuli ${ }^{73}$. 
Figure 6. Inhibitor of $\mathrm{KB}$ Kinase (IKK) pathway in the activation of NFKB

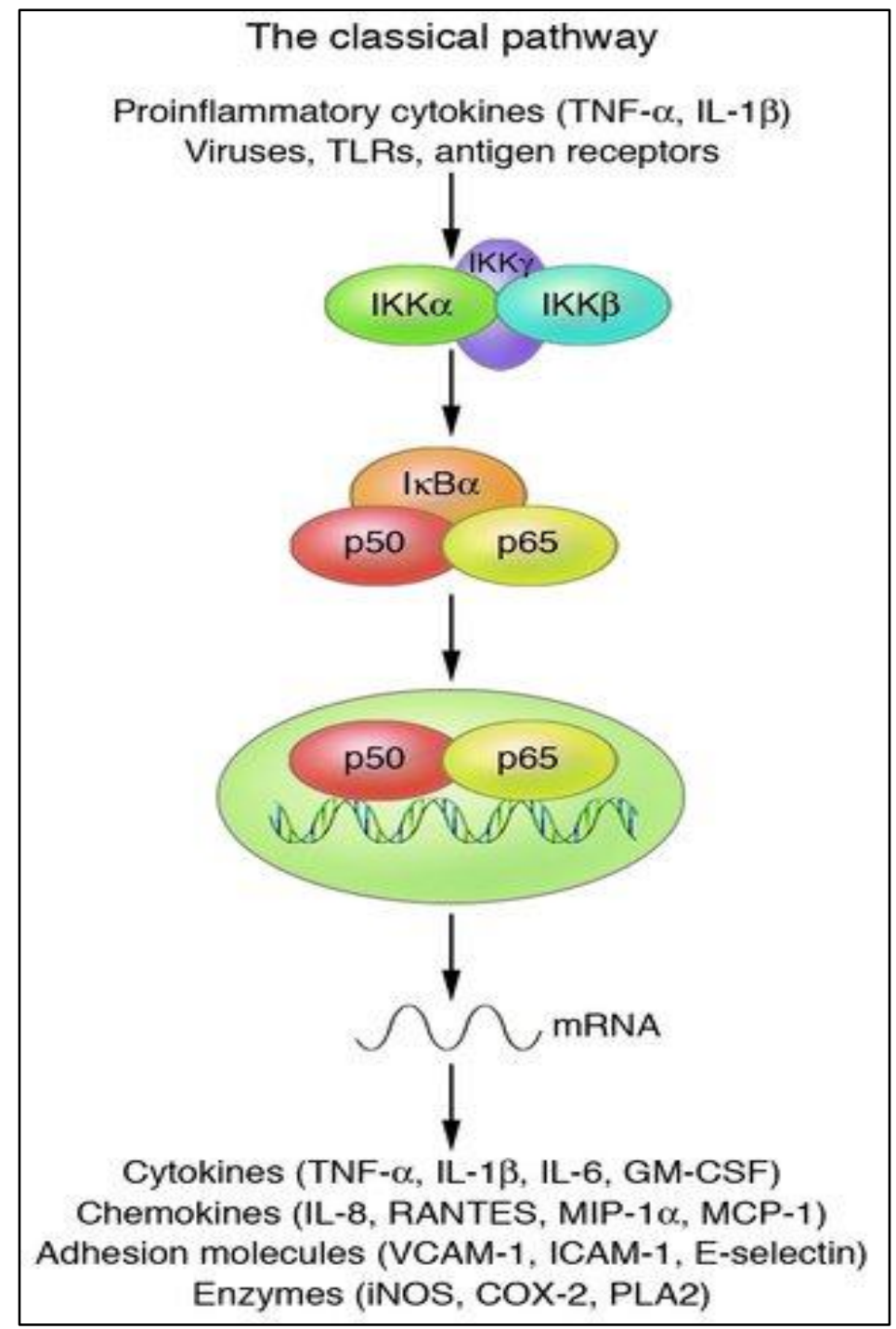

The classical pathway of IKK is activated by various DAMPs and PAMPs such as LPS. Phosphorylation of the IKK- $\alpha$ and/or $-\beta$ subunits activates the IKK complex, which directly phosphorylates $I K B \alpha$. This leads to the degradation of these "brakes" releasing the p65/p50 heterodimer (the key active canonical NFKB subunits) to become phosphorylated themselves and translocate the nucleus for the transcription of pro-inflammatory genes.

(Adapted from Luo et al, Journal of Clinical Investigation, $2005^{74}$ ). 


\section{Clinical implications of IKK}

Numerous studies over the years have investigated the dysregulation of cytokines that are dependent on IKK \& NFKB, such as TNF- $\alpha$

and IL-6, which are predictive of poor outcomes in surgical patients ${ }^{28,31}$. More recent studies have shown that excessive early NFKB stimulation is predictive of mortality in CLP models and predictive of systemic inflammatory response syndromes (SIRS) following major elective surgery ${ }^{75,76}$. These differences in the magnitude of activation may be surrogate markers for excessive early stimulation of IKK, upstream to NFkB in this pathway, however, few studies have examined IkK activation itself in surgical patients.

The Inflammation and the Host Response to Injury studies have been discussed earlier. In such high throughput genomic studies where thousands of mRNA levels are measured sequentially, reports did reveal some significant changes in IKK mRNA levels in trauma patients. Specifically, Laudanski et al examined cell-specific mRNA expression in monocytes and Tcells isolated from trauma patients ${ }^{37}$. IKK- $\beta$ was downregulated and IKK- $\varepsilon$ was upregulated in the monocyte when compared to healthy controls. Upstream of IKK, NIK was also upregulated. T-cells demonstrated a similar pattern of IKK- $\beta$ downregulation. IKK- $\alpha$ was not different and IKK- $ү$ was upregulated compared to healthy controls.

Various studies of in-vitro and in-vivo models have demonstrated IKK phosphorylation in response to LPS and CLP, respectively ${ }^{77}$. There has been increasing attention towards IKK blockers as a potential therapeutic target to prevent excessive inflammation. Of interest, one IKK 
blocker, SAR113945, was used in a phase II trial via intra-articular injection for osteoarthritis in Germany (clinicaltrials.gov).

In 2013, Coldewey and colleagues used another IKK blocker $(\mathrm{IKK}-16)$ in a mouse model of peritonitis ${ }^{78}$. They found that IKK-16 was effective in preventing the degradation of $I_{k B}$, limiting inflammation and decreasing lung injury caused by the infection. A group from China in 2014 found that IKK-16 was effective in reducing end organ failure in a rat model of ventilator-assisted lung injury ${ }^{79}$.

More recently, Sordi et al used IKK-16 by intraperitoneal injection in a rat model of hemorrhagic shock. The phosphorylation of IKK was successfully inhibited, with corresponding decreases in pro-inflammatory cytokines. Treated rats had reduced lung injury, and also renal and hepatic markers of organ failure were lessened ${ }^{77}$. In 2016, the same group reported the application of IKK-16 in a murine model of sepsis, using both LPS and CLP, with pre-existing renal impairment (by nephrectomy). This demonstrated improvements in sepsis-associated myocardial dysfunction ${ }^{80}$.

One lesson from history tells us that the therapeutic approach to immune modulation should not aim to simply prevent or "turn off" inflammation as this may have unrecognized harmful effects. Li et al examined the effect of IKK inhibition on other aspects of host defenses in a mouse model of CLP ${ }^{81}$. The benefits of decreased pulmonary edema were seen with IkK inhibition, and treatment of cultured macrophages did not negatively influence phagocytosis of bacteria or decrease colony forming units in blood, lung or peritoneal fluid. Mortality, however, was unchanged. 
In general, IKK appears to be a critical step in mounting a sufficient inflammatory response to an infective challenge. However, excessive stimulation appears to lead to exaggerated release of cytokines and downstream organ dysfunction. These undesireable consequences can be prevented in surgically relevant animal models of sepsis and shock, which makes IkK-16 an attractive therapeutic approach. However, we do not know how IKK function is affected in the context of monocyte impairment, where immune suppression is part of the pathophysiological problem. In addition, we do not understand the exact role of IKK in cytokine and antigen presentation functions, and whether the same benefits of IKK modulation will be seen when translating this approach to the human inflammatory response.

In summary, the monocyte is a master regulator of the immune response following major insults to the body through its important roles in cytokine production and antigen presentation. The functions of these roles are influenced by a variety of patient factors and by various clinical scenarios. There is a significant body of evidence that major physiological insults such as trauma can lead to excessive depression to the responsiveness to LPS and HLA-DR expression which results in an increased risk of infection and death. The function and role of IKK in monocyte impairment is not clear. We believe this complex and clinically relevant phenomenon is worthy of detailed study.

Please note that the text of this chapter has been published in a review. ${ }^{8}$ 
CHAPTER III

PAST, PRESENT AND FUTURE OF AUGMENTATION OF MONOCYTE FUNCTION IN THE SURGICAL PATIENT

\section{a. Background}

Sepsis is a clinical condition in a patient that has changes in systemic parameters combined with a proven source of infection. However, the underlying pathophysiology occurs in response to the dissemination of bacteria or other PAMPs/DAMPs into the bloodstream. This stimulates toll-like receptors to produce an inflammatory response, governed NFKB and MAPK p38 pathways to promote cytokine and chemokine release that results in the clinical signs and symptoms that many junior physicians recognize. A very similar response is seen following major trauma. Through the "Surviving Sepsis Campaign" there has been increased prompt recognition of the early phases of sepsis, with the aim of early goal-directed therapy such as titrated fluid boluses, appropriate intravenous antibiotic administration and closer monitoring of hourly urine outputs and serial lactate measurements. However, the later phases of a major insult such as major trauma, burns, or major infection that may include components of immunosuppression remain oblivious to many clinicians. The simultaneous down-regulation of antigen presenting genes and those pertaining to adaptive immunity may result in 
reduced HLA-DR expression, decreased TNF- $\alpha$ production in response to lipopolysaccharide (LPS) exposure and also T-cell anergy. This phenomenon, discussed in the previous chapter, does not yet have as widely accepted corrective or adjunctive therapy, although numerous credible laboratories pursue the subject in their own ways.

A major flaw in many of these studies, including our group's previous work, is the failure to define what is the most appropriate threshold in the laboratory to define impaired monocyte function and the most meaningful duration of that impairment. While low monocyte HLA-DR expression is commonly cited as the most commonly used predictive marker of surgical infection, reports have been variable in part due to a lack of standardization between laboratories. Newer methods of calibration to calculate the number of HLA-DR antibodies per cell have somewhat improved upon this problem ${ }^{82,83}$.

Various studies have aimed to boost immune function by increasing the proportion of normal, activated monocytes (such as with interferon-gamma, GM-CSF or other agents), but this has failed thus far to consistently show a reduction in mortality ${ }^{3,84,85}$. One of the biggest challenges has been in defining and identifying this subset of patients, which has in parallel stimulated the field of endotoxin tolerance research ${ }^{5,7}$. This principle is illustrated in Figure 7, where the monocyte previously stimulated by LPS has a decreased responsiveness to a subsequent LPS challenge. The role of potential therapy is demonstrated in Figure 8, an in-vivo model of endotoxin tolerance. Here, one can see the augmentation of TNF- $\alpha$ production when interferon-gamma is given subcutaneously. This chapter aims to address the evidence and lessons learned from previous trials in therapies aimed at restoring monocyte function, 
and to discuss current and future approaches to this clinically important conundrum. 
Figure 7. Principle of in-vitro studies of the impaired monocyte.

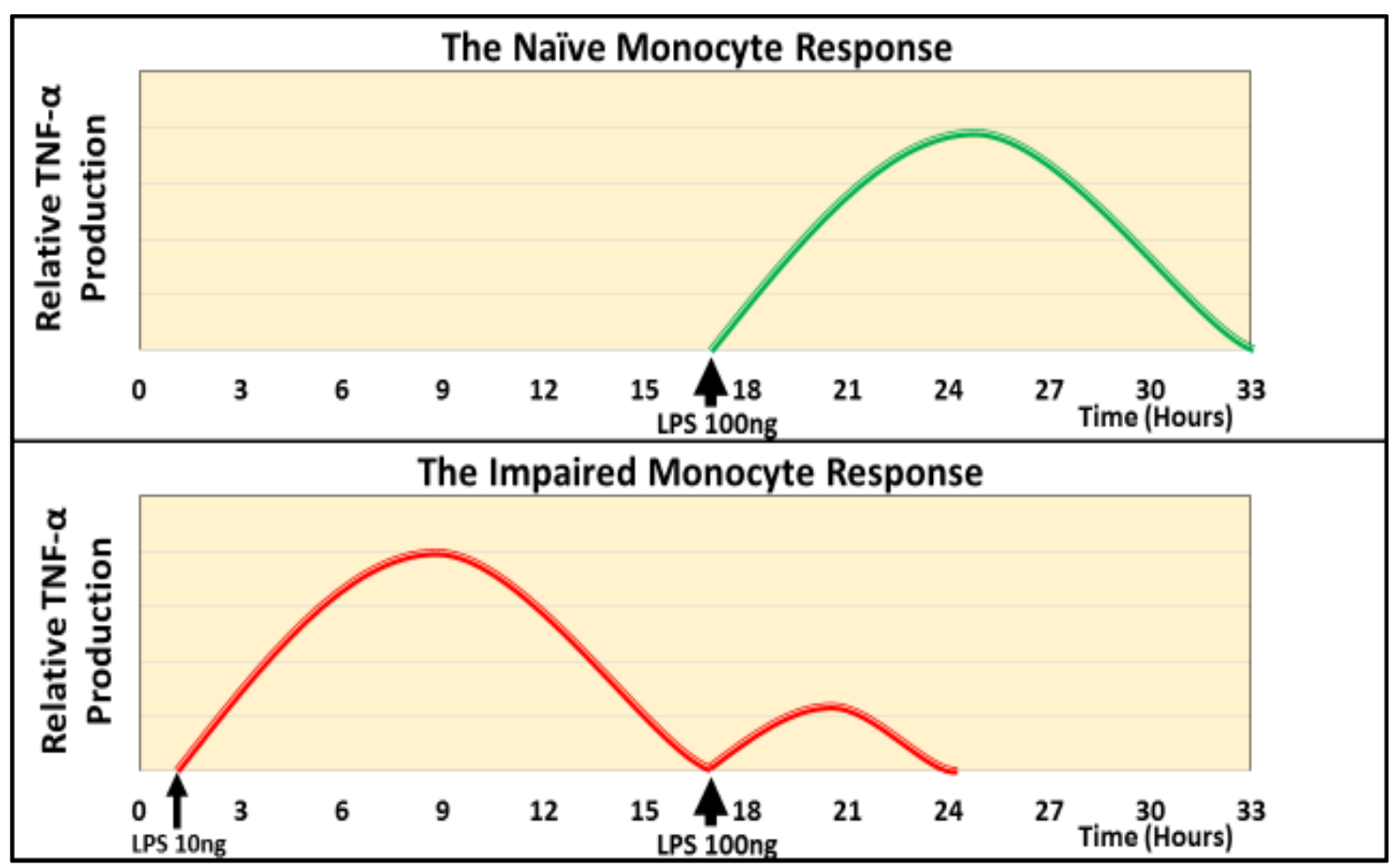

The naïve monocyte with no previous exposure to endotoxin represents a healthy response to a challenge, such as $L P S$, with a high level of TNF- $\alpha$ production (seen in green). By comparison, the impaired monocytes with early, low dose exposure to LPS mount an initial response characterized by high TNF- $\alpha$ levels but then becomes refractory to a subsequent LPS challenge, characterized by a blunted, lower level of TNF- $\alpha$ levels (seen in red) when compared against its naïve counterpart. 
Figure 8. Human in-vivo monocyte impairment.

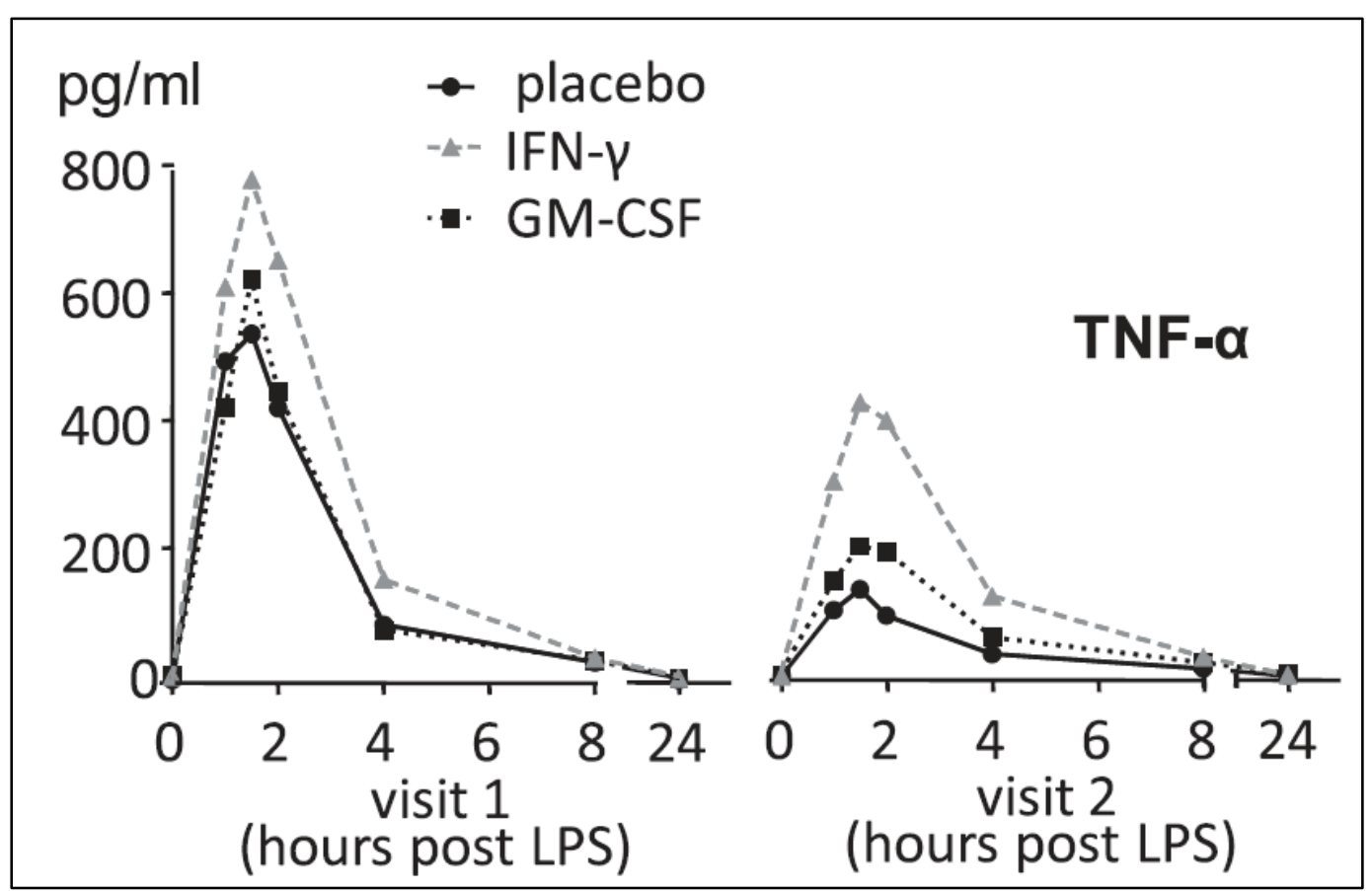

This figure demonstrates human in-vivo monocyte impairment in response to subcutaneous LPS administration. Compared to the first LPS dose (day 1), there is decreased monocyte responsiveness with decreased TNF- $\alpha$ production demonstrated above, to a second dose (day 7). Note partial reversal with IFN-y \& GM-CSF, when given between doses.

(Adapted from Leentjens et al, Am J Respir Crit Care Med, $2012{ }^{86}$ ). 


\section{b. Clinical evidence for therapies:}

\section{IFN-Y}

Polk et al, Am. J. Surg. 1992 (Trauma) $^{85}$

IFN-y has safely been used in humans for other clinical indications such as malignancy. A pilot clinical trial of 15 patients who were treated with IFN-y following major trauma demonstrated increased monocyte HLA-DR expression, albeit in a narrowly focused study. This was followed by a larger prospective randomized controlled trial of 201 patients enrolled with an injury severity score over 20 with bacterial contamination. Recombinant IFN-y (100 $\mu \mathrm{g}$ ) was given subcutaneously for 10 consecutive days, among 4 medical centers. HLA-DR expression was augmented for the duration of IFN-y treatment; however, levels quickly returned to that of the placebo group after treatment cessation at day 10 (Figure 9). Patients were followed up to 90 days and it was noted that a quarter of infections occurred after day 11. Fewer patients required an operation for infection and mortality appeared lower in the IFN-y treatment group, although this was not statistically significant. Fever was experienced in $93 \%$ of treated patients as a side effect of therapy. The authors later concluded that future trials might consider administering doses on alternate days over a longer duration, with the aim of reducing febrile side effects yet extending therapy ${ }^{87}$. Interestingly, this was a keystone of concurrent parallel work by Dries et al ${ }^{84}$. 
Figure 9. Effect of IFN- $y$ on monocyte HLA-DR expression in severely traumatized patients.

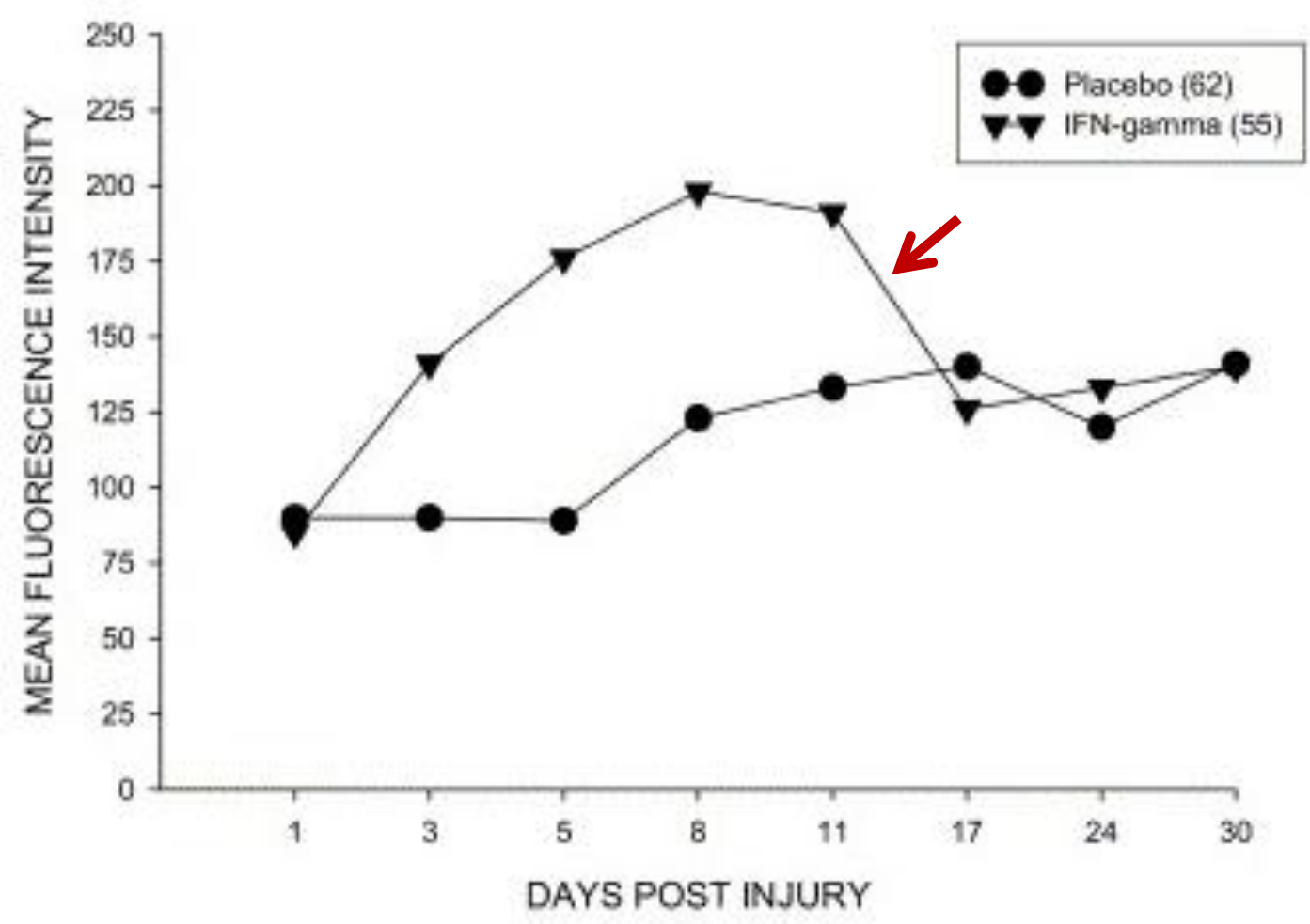

For the duration of treatment, the interferon- $y$ treated group (inverted triangles) benefit from increased monocyte HLA-DR expression (mean fluorescence intensity) compared to the placebo group (circles). However, note the rapid return of monocyte $H L A-D R$ to a similar level to the placebo group after the final dose of the regimen (given on day 10 post-injury, see red arrow).

(Adapted from Polk et al, Am. J. Surg., $1992^{85}$ ). 


\section{Dries et al, Arch. Surg 1994 (Trauma) ${ }^{84}$}

A second randomized controlled trial was undertaken in parallel, with 416 patients across 9 centers randomized to IFN-y or placebo. Again, severe trauma was the indication, but the major difference in design was that doses were given on alternate days, for 20 days rather than 10 days. The differences between this and the previous trial are discussed at length elsewhere ${ }^{87}$. The major findings were that all-cause mortality was similar, but infection related deaths were lower in the treatment group (3\% IFN-y vs. 9\% placebo). The authors noted an unusually high mortality rate in the placebo group in a single hospital that contributed this large imbalance. Interestingly, the treatment group did have a longer ICU stay than placebo, perhaps attributable to investigation of the febrile reaction to therapy. The excess death rates in the placebo arm in the leading enrolling institutions has not been solved intellectually or technically.

\section{$\underline{\text { Docke et al, Nature Medicine, } 1997}$ (Sepsis) $^{3}$}

In 1997, Docke et al used IFN-y in 9 patients diagnosed with sepsis and impaired monocyte function. This "monocyte deactivation" was defined as patients with less than 30\% HLA-DR positive monocytes for 2 consecutive days, in the context of Bone's criteria for sepsis ${ }^{88}$. Therapy was continued until HLA-DR expression was restored for 2 consecutive days, or 8 days total. Therapy also boosted ex-vivo TNF-a production when compared to 26 historical controls. This novel approach appeared to help clear infection in 8 of the 9 patients included. The mortality rate for the treatment group was reduced from $58 \%$ in similar patients to $33 \%$, this study was too small to draw 
conclusions on mortality. This approach of treating only patients with evidence of monocyte impairment was promising for larger studies. These authors appear not to have performed or contributed to other trials to enhance the power of this 9-patient trial.

\section{Wasserman et al, Crit Care Med, 1998 (Burns) ${ }^{89}$}

This phase III randomized double-blinded trial compared subcutaneous IFN-y in 109 burn patients compared with 107 patients receiving placebo. These patients had severe burns, scoring at least 7 using the abbreviated burn index. Therapy was started in the first 4 days after thermal injury and continued daily until 90 days, hospital discharge, or wound closure. IFN-ץtreated patients had equivalent rates of major infection and death. The putative benefits of therapy could have been masked by including patients with no evidence of monocyte impairment and the inclusion of older, severely burned patients, likely to die regardless of intervention.

\section{Nakos et al, Crit Care Med 2002 (Trauma) $^{90}$}

In 2002, Nakos et al studied mechanically ventilated trauma patients with a decreased alveolar macrophage HLA-DR of less than $30 \%$, obtained through bronchoscopy (compromising 21 of 52 patients). Patients were randomized to inhaled recombinant IFN-y (11 patients) versus placebo (10 patients). Injury severity score was 40 and 43 respectively in these groups. The treatment group received inhaled IFN- $y$ three times a day. Investigators found HLA-DR expression increased from a $16.4 \%$ to $44 \%$ in the follow up bronchoscopy on day 3 post injury. This compared with persistence of low HLA-DR in the placebo group. In addition, there was a decrease in IL-10 
concentration in bronchoalveolar fluid and decreased rates of ventilatorassociated pneumonia compared to placebo. It is not clear whether the inhaled delivery allowed IFN- $y$ to have significant systemic effects. Mortality remained unchanged. This important trial needs to be repeated; the study of alveolar macrophages with inhaled IFN- $\gamma$ "therapy" is unique.

Schinkel et al, Shock, 2001 \& Licht et al, J. Interfer. Cytoki. Res. 2003

\section{(Major elective surgery) ${ }^{91,92}$}

The Faist group (both papers) screened 209 patients undergoing major surgery for pre-operative immunosuppression, as defined by a negative delayed type hypersensitivity skin test ${ }^{91}$. Of these, 46 anergic patients (22\%) were randomized to pre-operative parenteral IFN-y administration $(-7,-5$, and -3 pre-operative days). Visceral, gynecological, vascular, and thoracic operations were included and were said to be physiologically equivalent in each group. There was a significant increase in peripheral monocyte HLA-DR, serum IL-1 and IL-8. LPS induced TNF- $\alpha$ was also augmented. A subsequent report from the same group in 2003 reported that treated patients demonstrated increased IL-2R levels and decreased IL-4 levels, without an effect on IL-10 or lymphocyte proliferation, suggesting a shift to a Th1 phenotype. The authors cited a generally low number of infectious complications and were unable to demonstrate any real differences in morbidity and mortality. There are many examples of bias in these trials of dually reported patients that could warrant repeating the trial in more depth and breadth, particularly to the extent that skin testing does or does not reflect monocyte function. 


\section{$\underline{\text { GM-CSF }}$}

Meisel et al, Am J Respir Crit Care Med, 2009 (Sepsis) ${ }^{93}$

This prospective, randomized double blinded trial using granulocytemacrophage colony stimulating factor (GM-CSF) was undertaken in very ill patients, requiring the presence of severe sepsis or septic shock for inclusion. More specifically, patients were required to have depressed monocyte HLADR expression for two consecutive days. This yielded 38 patients from a total of 135 who were screened for impaired monocyte function. Pneumonia was the leading cause of sepsis, accounting for $60 \%$ of the 38 patients, while peritonitis was responsible for $30 \%$. Following 8 days of treatment, all 19 patients in the treatment arm demonstrated increased monocyte HLA-DR expression, as well as restored ex-vivo TNF- $\alpha$ secretion. Furthermore, increased T-cell and monocyte counts increased, accompanied by a decrease in IL-10. Such patients had shorter time of ventilation and shorter ICU stays, however mortality was $16 \%$ in the GM-CSF group vs. $21 \%$ in the placebo group, this did not reach statistical significance.

Leentjens et al, Am J Resp Crit Care Med, 2012(Experimental endotoxemia) ${ }^{86}$

Leentjens et al successfully induced impaired monocyte function in healthy volunteers with low dose intravenous LPS. Eighteen males were randomized to receive IFN- $\gamma$, GM-CSF or placebo for 3 alternate day doses, followed by a second LPS dose on day 7 . The placebo group had an initial increase in the cytokine levels of TNF- $\alpha$, IL-6, IL-10 and IL-1RA after the first LPS dose, but after the second dose a reduction in TNF- $\alpha$ production was seen, in keeping with in-vitro monocyte models (Figure 8). Following the 
second LPS dose, there was a trend of lower IL-10 but this did not reach statistical significance. HLA-DR expression decreased following the first dose of LPS, but appeared to recover prior to a subsequent similar depression in response to the second LPS dose. Compared to placebo, IFN- $y$ increased TNF- $\alpha$ and HLA-DR, with decreased IL-10, and was more effective than GMCSF in achieving monocyte activation.

\section{Spies et al, PLoS One, 2015 (Major Elective Surgery) ${ }^{94}$}

This recent randomized controlled trial examined the role of continuous intravenous infusion of GM-CSF in elective esophageal and pancreatic resections in preventing post-operative infection. Crucially, only patients with empirically proven low monocyte HLA-DR expression measured on postoperative day 1 were eligible. While we have discussed using a single observation to trigger immunoadjuvant therapy, more than one subnormal value could ascertain the deficiency more clearly. Sixty-one patients in total were included, equally distributed into a GM-CSF group, influenza vaccination and placebo. As expected, the GM-CSF treated group developed significantly higher monocyte HLA-DR expression compared with placebo. However, daily HLA-DR measurements were used to assess the response and as soon as the treated patients reached the threshold of 10,000 monoclonal antibodies (mAb) per cell, therapy was stopped. Therefore, duration of this immunoadjuvant was short, lasting between 1-3 days of continuous intravenous infusion. The result of this was a rapid return of monocyte HLADR to the depressed levels of the other two groups by post-operative day 5 . Note the similarity of this "post-treatment dip" to our group's prior interferongamma study, as demonstrated in Figure 9. Spies et al reported a shorter 
number of "infection days" in the GM-CSF treated patients, but the postoperative infection rate was similar between groups. The learning point may be that single monocyte HLA-DR measurement of response may not be an optimum marker of treatment effect. In addition, the immunological benefits from GM-CSF appear transient (at least in circulating monocytes) and therefore therapy probably should continue to at least 7-14 days based on several other studies when the peak of post-operative infection rates might occur.

The decision to include an arm of influenza vaccine and its apparent association with increased delirium detracts from the clarity of this work.

\section{$\underline{\text { G-CSF }}$}

$\underline{\text { Schneider et al, Ann. Surg, } 2004} \underline{\text { (Major Elective Surgery) }}^{95}$

Prophylaxis with granulocyte colony stimulating factor (G-CSF) was given pre-operatively in patients undergoing major extirpative surgery. Sixty patients were divided equally into three arms; two different G-CSF regimens and placebo. In the first regimen, subcutaneous G-CSF was given on perioperative days $-2,1$ and 3 , compared with the second regimen with a dose on day -2 , with smaller subsequent doses on the following 5 days. Operations included colectomy, gastrectomy, Whipple pancreatoduodenectomy, esophagectomy, and liver and lung resection. Patients who received G-CSF had increased TNF- $\alpha$ production, HLA-DR expression and exhibited decreased lymphocyte anergy. This led to both 
decreased rates and severity of infection in the treatment group. Interestingly, the authors stated that, in their opinion, monocyte deactivation was irreversible, and that this therapy exerted its effects by increasing the functioning number of leukocytes. While this study included an odd collection of extensive operations, the differences described are worthy of future work.

\section{Bo et al, L. Crit Care 2011 (Sepsis) $^{96}$}

In 2001, Bo et al undertook a meta-analysis of 12 RCTs using either GCSF or GM-CSF in sepsis. This included a total of 2380 patients. The vast majority of studies included did not target therapy specifically for those with impaired monocyte function. While this study demonstrated a trend of lower deaths in the treatment groups, there were no statistically significant differences in 28-day mortality for either immunoadjuvant. That being said, in three trials of G-CSF and one trial of GM-CSF, both agents appeared to significantly reverse infection.

\section{Alternative approaches}

While beyond the scope of this chapter, there have been various alternative approaches for immunomodulation even including extra-corporeal blood and plasma filtration. Early work has shown that endotoxin filtration combined with immune stimulation improves survival in a murine model of sepsis ${ }^{97}$. Endotoxin filtration, such as with polymyxin B cartridges, have been accepted therapy in Japan in severe sepsis with approval also for many years in Europe, as discussed by Esteban et al ${ }^{98}$. Furthermore, cytokine filters 
(such as CYTOSORB ${ }^{\circledR}$ ) have the potential for filtering "harmful" proinflammatory cytokines. This principle is probably oversimplified, and elucidating optimum timing, if any, will be very difficult. Nevertheless, there is not yet acceptable evidence at present for its use in USA.

\section{c. Challenges of therapy}

Providing the patient with a reliable immunoadjuvant to treat the underlying pathophysiology of a major insult, whether it specifically addresses monocyte impairment or other aspects of the response, remains an old conundrum yet to be resolved.

Spontaneous insults such as major trauma, burns or sepsis are a difficult issue to control for in a prospective, randomized fashion. With such heterogeneity, ensuring equal distribution between treatment and placebo groups is difficult, as discussed. Furthermore, each patient will have numerous treatments ranging from antibiotics, fluid therapy, inotropic support and potentially mechanical ventilation, not to mention source control. With so many variables, proving that the addition of a single adjuvant can make a statistically significant improvement in mortality becomes difficult.

Many studies may initially show improvement with short term outcomes, yet when one examines 90 day mortality it often remains unchanged ${ }^{99}$. Timing is a major issue not just for follow up, but also with the introduction, duration and cessation of therapy given the changing immune milieu from initial insult to prolonged recovery as discussed earlier. 
The immune system is redundant, which is likely a product of evolution. With so many different positive and negative effects of various inflammatory pathways, changing or altering one protein is not likely to ultimately change the outcome for a given patient. Indeed, it may have unrecognized negative consequences, such as those seen in early corticosteroid and anti-TNF- $\alpha$ therapeutic trials. Compare this, however, with the common use of brief perioperative antibiotic prophylaxis for clean-contaminated elective operations. Antibiotics were in use by surgeons for more than 20 years before definitive clinical proof was accomplished; this is now the most common use of antibiotic agents in the world. In retrospect the persuasive experiment was the pre-operative antibiotic dose ${ }^{100}$.

Although not specifically aimed at monocyte function, a meta-analysis of the effects of immune adjuvants in the context of sepsis is shown in Figure $10^{101}$.

Early approaches, specifically for sepsis, targeted the apparent exuberant pro-inflammatory response, the so called "cytokine storm." The rational for a therapeutic target was the predominance of TNF- $\alpha$, IL-6 and IL$1 \beta$ production from innate immune cells such as monocytes, leading to endothelial activation and the release of acute phase proteins contributing to vasodilation, shock, acute respiratory distress syndrome and multi-organ failure. Furthermore, blockage or binding of LPS may be able to lower the PAMP load to limit the foregoing cascade. A simple "dampening" of the immune system with corticosteroid therapy has remained controversial. Moreover, this approach has never convincingly improved mortality. The only FDA approved sepsis therapy was detracogin alpha (Xigris $\AA$ ), a form of 
activated protein C. Despite the initial success of the PROWESS trial, concerns for its efficacy and bleeding side effects lead to the subsequent PROWESS-SHOCK trial in 2012. Those investigators concluded that 28 and 90 day mortality were not significantly different, leading to subsequent withdrawal of FDA approval ${ }^{99}$. 
Figure 10. Therapeutic efficacy of mediator-targeted therapy for sepsis.

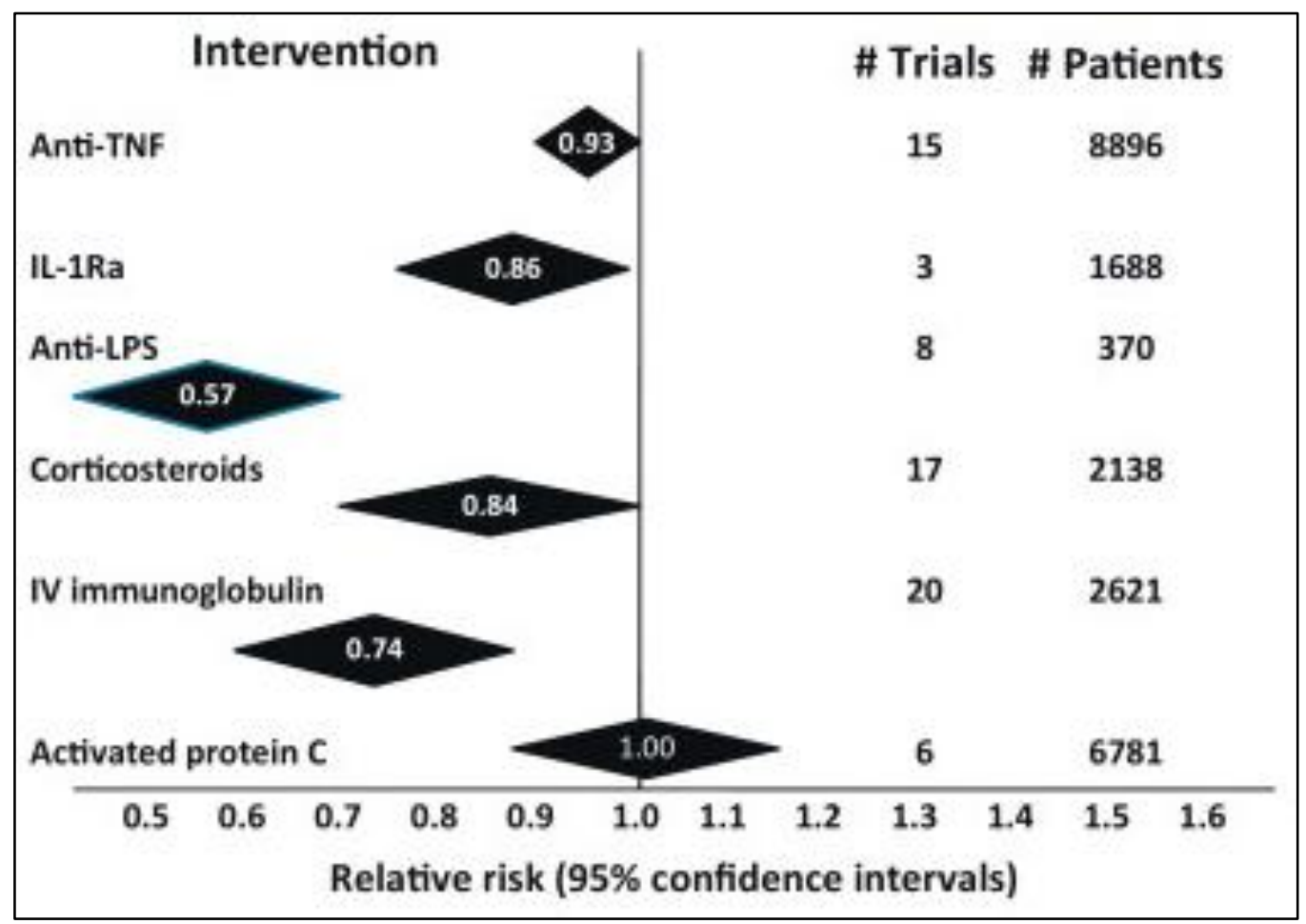

Therapeutic efficacy of mediator-targeted therapy for sepsis. This metaanalysis demonstrates relative risks and confidence intervals of various immune modulators are plotted based on the probability of death compared to the untreated controls. A relative risk of 1 indicates no difference in survival, and a relative risk of less than 1 indicates that the therapy has a decreased probability of death, thus is superior to untreated controls. Unfortunately, when many of these therapies were expanded to larger studies or different populations, the potential to improve mortality was not fulfilled.

(Modified from Marshall DC, Trends Mol. Med., $2013^{101}$ ). 
Eritoran is a synthetic lipid A antagonist to MD-2-TLR4 that was recently used in a randomized controlled trial of 1961 patients diagnosed with severe sepsis. This followed previous strategies targeting endotoxemia (such as lipid A antibodies). In this large phase III trial, the placebo group had a mortality of $27 \%$, much lower than the $40 \%$ anticipated by investigators. Furthermore, patients with gram positive infection actually appeared to do worse than placebo. In contrast to a smaller phase II trial preceding it, there was no decrease in 28-day or 1 year mortality ${ }^{102}$.

\section{d. The future of monocyte biology in the surgical patient}

Since the continued failure of clinical trials to show a mortality benefit between major trauma and sepsis, consensus in the literature seems to be moving towards ever more targeted therapy ${ }^{20,21,49,103}$. For example, treating only those patients with depressed monocyte HLA-DR with IFN- $\gamma$, rather than non-specifically treating all patients. Such a strategy surely reduces the denominator, thus enhancing the demonstrable positive effects of putative treatments.

This approach, therefore, needs a biomarker that would accurately represent the underlying immune phenotype, be present early enough to allow the benefit of therapy, and also respond to therapy in order to guide treatment response.

In many diseases, miRNAs have been found to be stable and reliable markers in various conditions, including cancer. Furthermore, various studies 
have identified miRNAs that are dysregulated in patients with sepsis ${ }^{104-107}$. More recent studies have implicated miRNAs in the host defense to trauma 108-110. In general, these studies have not been correlated to underlying monocyte phenotype. It remains unclear if these markers could fulfill some of our criteria. For example some miRNAs such as miRNA-133a have been linked to increased mortality in sepsis ${ }^{111}$. In-vitro studies using cell lines have shown that manipulation of such miRNAs could abrogate monocyte impairment; but as it stands there appears to be no immediate miRNA-based immunotherapy planned for human trials.

It has been postulated that negative co-stimulatory markers such as PD-L1 are increased in patients with sepsis. There are few trials demonstrating this phenotype in major elective surgery or trauma. However, the apparent success with anti-PD-1 \& anti-PD-L1 based therapy for malignant melanoma in humans may indicate that such therapy could be utilized for trauma or septic patients exhibiting this immune phenotype.

\section{e. Future immunoadjuvants}

It is not just PD-1 based therapies that are targeting T-cell defects in sepsis patients. Recent murine studies of sepsis models promise that IL-7 and IL-15 monoclonal antibodies increase the activity and function of T-cells. This may in turn increase endogenous IFN- $\mathrm{Y}$ and restore the healthy monocyte-Tcell interaction. However, much more research is required before this can reach human clinical trials. The current list of registered trials for trauma and sepsis relating to monocyte function is summarized in Table 4 (clinicaltrials.gov). 
Table 4. Current trials relating to monocyte function (clinicaltrials.gov).

\begin{tabular}{|c|c|c|c|c|c|}
\hline Trial number & Centre & Type & Setting & Intervention & Status \\
\hline NCT01649921 & $\begin{array}{l}\text { Radbound, } \\
\text { Netherlands }\end{array}$ & Interventional & Sepsis & IFN-y & Recruiting \\
\hline NCT02361528 & Lyon, France & Interventional & $\begin{array}{c}\text { Sepsis (\& low } \\
\text { HLA-DR) }\end{array}$ & GM-CSF & $\begin{array}{l}\text { Not yet } \\
\text { open }\end{array}$ \\
\hline NCT01653665 & Newcastle, UK & Interventional & $\begin{array}{c}\text { Sepsis (\& } \\
\text { PMN <50\%) }\end{array}$ & GM-CSF & Completed \\
\hline NCT01495637 & Ohio, USA & Interventional & $\begin{array}{c}\text { Pediatric } \\
\text { Trauma }\end{array}$ & GM-CSF & Recruiting \\
\hline NCT01161745 & $\begin{array}{l}\text { Changhai, } \\
\text { China }\end{array}$ & $\begin{array}{c}\text { Observational } \\
\text { (for PD-1/PD- } \\
\text { L1) }\end{array}$ & Sepsis & $\mathrm{N} / \mathrm{A}$ & Completed \\
\hline NCT02246595 & Jena, Germany & Interventional & Septic shock & СаCP29 & Recruiting \\
\hline NCT01378169 & Paris, France & $\begin{array}{c}\text { Observational } \\
\text { (Monocyte } \\
\text { microRNA) }\end{array}$ & Sepsis & $\mathrm{N} / \mathrm{A}$ & Unknown \\
\hline NCT02576459 & $\begin{array}{l}\text { Bristol-Myers } \\
\text { Squibb, Site not } \\
\text { specified, USA }\end{array}$ & Interventional & Septic shock & Anti-PD-L1 & $\begin{array}{l}\text { Not yet } \\
\text { open }\end{array}$ \\
\hline NCT02186522 & Edinburgh, UK & Observational & Sepsis & $\mathrm{N} / \mathrm{A}$ & $\begin{array}{l}\text { Not yet } \\
\text { open }\end{array}$ \\
\hline
\end{tabular}

Last accessed July, 2016. 


\section{f. Conclusion}

The predisposition and response of the immune system as a factor in the outcome of the surgical patient remains as relevant in 2016 as it ever has. As high-throughput systems approaches to trauma and sepsis, such as the Inflammation and the Host Response to Injury Project, continues we recognize an increased level of complexity with more and more molecules associated with poor outcomes such as nosocomial infection and indeed death. The field has progressed well beyond a rudimentary pro- or antiinflammatory response to a given insult. Yet the same challenges remain. With this increased knowledge, how can the physician hijack the immune system to improve the outcome of a patient? The question of what therapy might be optimum, and the timing and duration of this, remains.

In a previous chapter, it was discussed how there may be different components to a patient who has impaired host defenses, and indeed one biomarker, or therapy may not fit all. For example, perhaps interferon-gamma or GM-CSF is the right adjuvant for the patient with proven impaired monocyte function, and anti-PD-1 therapy might be appropriate for the patient with proven T-cell anergy with elevated PD-1 expression. In this chapter, one of the most promising agents is still interferon-gamma, but the persuasive trial that is adequately powered and targeted to those with impaired monocyte function has not been done. Much of the failed translation of pre-clinical studies to human trials for certain therapies may be in part due to the limits of murine research, the complex genetic and post-translational variation in our patients, and an incomplete understanding of how our very redundant immune system responds. 
At present, there is insufficient evidence for the use of interferongamma, G-CSF or GM-CSF in the adjuvant setting for major trauma, surgery or overt infection. Furthermore, new therapies, with apparent success in the oncologic setting, have yet to be trialed. Currently registered trials discussed here will hopefully answer some of these questions. We believe that while mortality has yet to be convincingly reduced with the use of such agents, the approach of targeting patients with proven monocyte impairment for the right duration, whatever that might be, holds potential in improving outcomes in some of our patients.

In summary, this chapter has shown that corrective therapy to restore monocyte function in surgical patients has gained considerable traction for 30 years, but no convincing improvements in mortality rates have been demonstrated. Furthermore, these clinical considerations highlight the incomplete understanding of how the immune response can become "maladaptive". In order to achieve the ultimate goal of reliably identifying patients with impaired monocyte function and to correct this defect to improve outcomes, there is a need for better understanding the mechanisms by which the impairment takes place. Therefore, the work in this dissertation focuses on understanding how an insult such as endotoxin exposure might change intracellular signaling and lead to a suppression of the human monocyte inflammatory response.

Please note that the text of this chapter has been published in a review. ${ }^{9}$ 


\section{CHAPTER IV}

\section{HYPOTHESIS, SPECIFIC AIMS AND EXPERIMENTAL PLAN}

\section{A. Key Objective}

To develop a clinically relevant in-vitro model of monocyte impairment and study the defects in underlying signaling that lead to the suppression of proinflammatory cytokine production which may predispose critically-ill surgical patients to nosocomial infection and death.

\section{B. Hypothesis}

LPS induced monocyte impairment occurs through sustained feedback inhibition of IKK, leading to decreased TNF- $\alpha$ production, also represented by decreased HLA-DR expression \& increased IL-10 production.

This dissertation focuses on TNF- $\alpha$ as a valid marker of monocyte function.

\section{Specific aims}

1. Develop a model of monocyte impairment, in which low dose endotoxin exposure leads to altered cytokine responses, analogous to that seen in some trauma and complex surgical patients.

2. Investigate whether decreases in the cytokine response of the impaired monocyte is due to inhibition of IKK, leading to subsequent decreases in NFKB activation. 
3. Determine which cytokines other than TNF- $\alpha$ and IL-10 are impaired, and determine the influence of IKK on their production.

\section{Experimental Plan}

The purpose of this project was to develop a model of monocyte impairment using isolated human monocytes. We anticipated that the in-vitro model would allow monocytes to have suppressed cytokine responses, specifically decreased TNF-a production, and decreased HLA-DR expression, recapitulating the defect seen in the critically-ill surgical patient.

We then examined the underlying signaling mechanisms, starting with differences in TNF- $\alpha$ gene expression (mRNA) between naïve monocytes and those which are impaired. We then worked proximally in the signaling pathway to examine the differences in NFKB function and then IKK function. We anticipated decreased activation of both complexes of this important pathway in the impaired monocyte. This was indeed the case, therefore we then examined upstream circuitry of IKK to investigate dysregulation in toll-like receptor (TLR) signaling as well as miRNA's which regulate this complex network. Finally, we took a global view of a wide variety of cytokine and chemokine levels to determine which other functions are suppressed as part of monocyte impairment, and the role of $\mathrm{IKK}$ in their production through its inhibition. 
Figure 11. Summary of hypothesis.
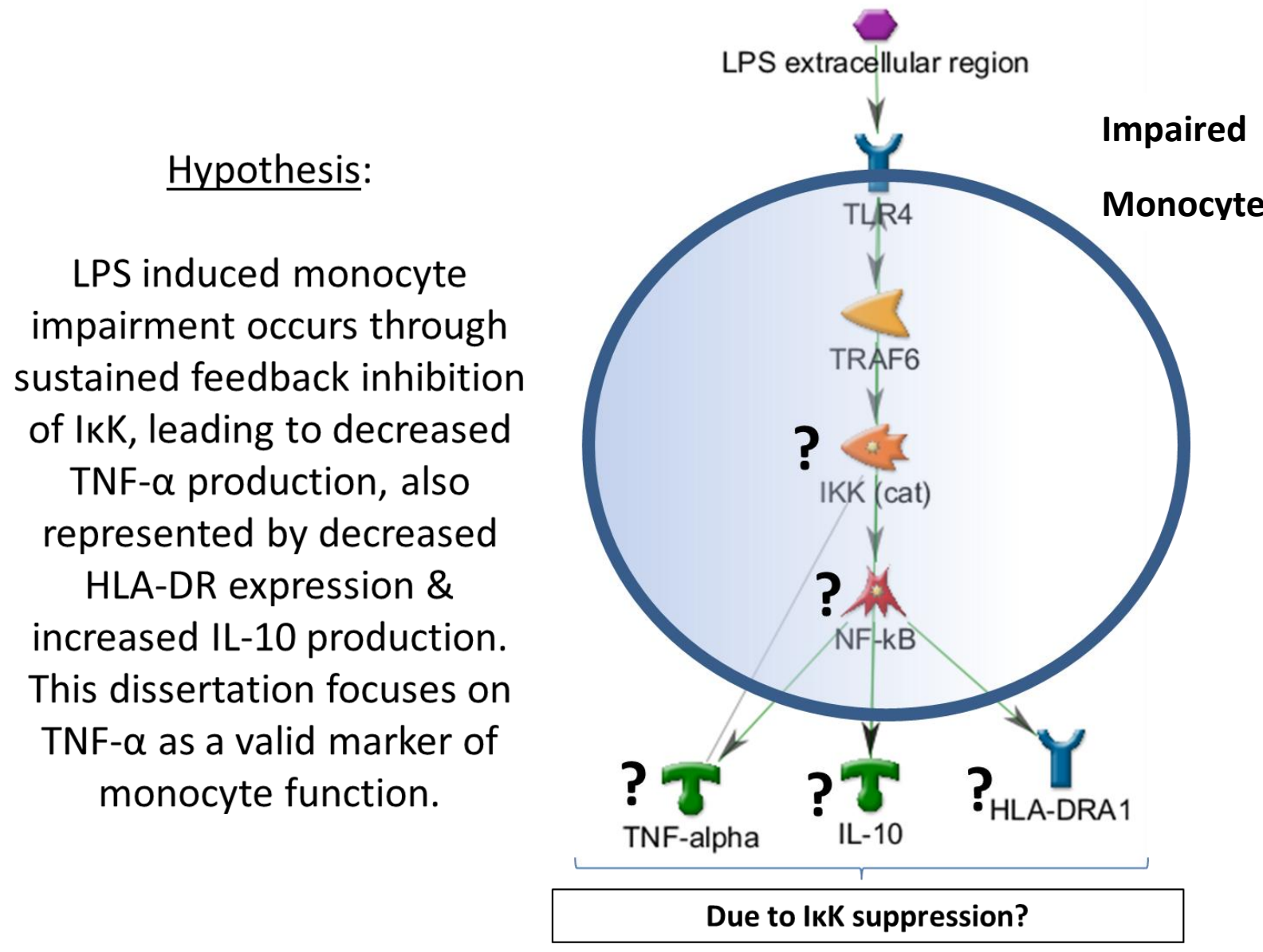


\section{e. Methods}

\section{i. Monocyte isolation}

Approval was given by the University of Louisville Institutional Review Board (IRB) (“Study of Immunological and Genetic Markers in Hospitalized and Ambulatory Subjects", 0.0018) and written informed consent was obtained from all donors. The age of donors ranged from $18-54$ years. A pool of 18 donors (10 male) in total were used for donation, although between 4 and 10 donors were used for a given experiment. Exclusion criteria included acute illness, anti-inflammatory medications, diabetes mellitus and any immunosuppressive conditions.

Up to $25 \mathrm{~mL}$ of venous blood was collected in EDTA Vacuntainer tubes (Becton Dickinson, Frankin Lakes, NJ). The collected blood was then incubated with CD14 magnetic microbeads and primary human monocytes isolated using a positive selection magnetic cell sorting technique according to manufacturer's instructions (Miltenyi Biotec, Auburn, CA). In brief, after 15 min incubation with the microbeads, cells were washed with MACS Separation Buffer, resuspended and run through magnetic columns. Columns were then washed three times to eliminate non-monocyte material, before cells were eluted with MACS Elution Buffer. Cells were then counted to check for total number, purity and viability of the isolate using Trypan Blue staining.

Primary monocytes were then cultured in 1640 RPMI medium (MP Biomedical, Solon, $\mathrm{OH}$ ) with 10\% heat-inactivated Fetal Bovine Serum, 2 nM L-Glutamine, antibiotic (100 IU/mL penicillin and $100 \mu \mathrm{g} / \mathrm{mL}$ streptomycin) and 
anti-mycotic (250 ng/mL amphotericin B). All the above were purchased from Thermo Scientific, Waltham, MA. Monocytes were cultured at $37^{\circ} \mathrm{C}$ in a humidified incubator at $5 \%$ carbon dioxide concentration. Cells were cultured either at $0.25 \times 10^{6}$ cells $/ \mathrm{mL}$ after initial isolation or $0.5 \times 10^{6}$ cells $/ \mathrm{mL}$ after resuspension at $17 \mathrm{hrs}$ depending on the experimental conditions. Fifty $\mathrm{mL}$ polypropylene tubes were used for these single cell suspension experiments (VWR, Westchester, PA).

Lipopolysaccharide (LPS) from E.Coli 0111:B4 was used for cell stimulation, at doses of either $10 \mathrm{ng} / \mathrm{mL}$ or $100 \mathrm{ng} / \mathrm{mL}$ depending on experimental conditions which were based on preliminary experiments (Sigma-Aldrich, St. Louis, MO).

\section{ii. In-vitro induced monocyte impairment}

To develop impaired monocytes, the following steps were undertaken; cultured cells from a given donor were divided into separate tubes and rested for 1 h. At 1 h, "naïve" monocytes were cultured in media only, however "impaired" cells were given low dose LPS $(10 \mathrm{ng} / \mathrm{mL})$ at this time. Cells were then cultured in parallel for a further $16 \mathrm{~h}$. At the $17 \mathrm{~h}$ time point, cells were centrifuged at $1800 \mathrm{rpm}$, counted and resuspended in fresh media. This step ensured that each condition had equal concentrations of viable monocytes for comparison. Cells at all conditions were then given a high dose, LPS challenge (100 ng/mL) to determine monocyte responsiveness. 


\section{iii. Cytokine assays}

Two series of cytokine assays were used to determine cytokine supernatant protein concentrations. Following cell culture incubations, tubes were centrifuged at $2000 \mathrm{rpm}$ for 10 mins to ensure an acellular supernatant. This supernatant was then aspirated and stored at $-80^{\circ} \mathrm{C}$ until subsequent analysis. For individual key cytokines, TNF- $\alpha$, IL-10 and IL-6 concentrations were quantified by enzyme-linked immunosorbent assay (ELISA) kits (eBiosciences, San Diego, CA). ELISA assays were run in 96 well plates in accordance with manufacturer's instructions. A standard curve was generated using recombinant TNF- $\alpha$, IL-10 or IL- 6 as appropriate. Activity was measured using a SpectraMax Plus384 spectrophotometer at a $450 \mathrm{~nm}$ wavelength. All samples were measured in duplicate.

For multiplex cytokine experiments, $25 \mu \mathrm{L}$ of supernatant sample were used per well in duplicate in pre-mixed 96 well plates using magnetic beads for 29 different cytokines and chemokines as per manufacturers recommendations (Milliplex Human Cytokine/Chemokine 29-Plex Magnetic Bead kit, EMD Millipore, Billerica, MA). Recombinant pre-mixed beads were used to create a standard curve. Quality controls $1 \& 2$ were used. Plates were run using a Luminex MAGPIX instrument (EMD Millipore, MA).

\section{iv. Flow cytometry}

Monocyte cell culture samples containing 100,000 cells were washed twice in Phosphate Buffered Saline (PBS) by centrifugation and resuspended in $100 \mu \mathrm{l}$ of PBS. Cells were stained with fluorescein isothiocyanate (FITC)-labeled anti-human $\mathrm{CD}_{14}^{+}$and phycoerythrin (PE)-labeled anti-HLA-DR antibodies 
(BD Biosciences, La Jolla, CA). Cells were stained for 25 minutes, before two further PBS washes and fixation in $300 \mu \mathrm{L}$ of $1 \%$ paraformaldehyde solution until subsequent analysis. Flow cytometric analysis was undertaken using a FACS Calibur flow cytometer (Becton Dickinson, San Diego, CA). A minimum of 3,000 gated events were acquired for each sample. Cell Quest software was used to determine cell purity (based on CD14 and HLA-DR positivity) and mean fluorescence intensity (MFI).

For phosflow analyses for determination of pathway activation by intracellular protein phosphorylation, 100,000 cells were fixed with $3.2 \%$ formalehyde and washed twice after culture with stain buffer (PBS containing fetal bovine serum $2 \%$ ) and permeabilized with $100 \%$ methanol for 30 min at $-20 \mathrm{C}$. Cells were then resuspended in $100 \mu \mathrm{L}$ stain buffer and incubated for $1 \mathrm{~h}$ in the dark with either phospho-p65 or phospho-p38 MAPK (BD Biosciences, La Jolla, CA). Finally, cells were then washed twice with stain buffer and run fresh within $2 \mathrm{~h}$ of stimulation using the FACS Calibur as described above.

\section{v. RNA isolation}

In order to obtain RNA, after a given time point of cell culture, cells were pelleted and stored in $300 \mu \mathrm{L}$ of lysis buffer at $-80^{\circ} \mathrm{C}$. RNA was extracted using the MirVana RNA extraction kit (Thermo Fisher Scientific, Waltham, $M A)$.

For quality control testing, RNA purity and concentration was measured using the Nanodrop N-1000 (Agilent Biosystems, Santa Clara, CA). Samples were only used if they fulfilled the quality control criteria of $1.8-2.2$ purity, based on the ratio of absorbance at $260 \mathrm{~nm}$ and $280 \mathrm{~nm}$ (A260/A280 
ratio). This excluded samples of poor, unreliable quality that were at risk of containing contaminating DNA, protein or other organic compounds.

\section{vi. mRNA measurements}

To determine the expression of individual genes of interest, RNA was diluted to $2 \mathrm{ng} / \mu \mathrm{L}$ concentration and used to synthesize complimentary DNA (cDNA) by reverse transcription (High Capacity cDNA Reverse Transcription kit, Life Technologies, Foster City, CA).

Quantitative real time polymerase chain reaction (qRT-PCR) was undertaken with Taqman® 20X specific mRNA primers, such as those for TNF- $\alpha$, using $18 S$ as an internal control. Samples were run in duplicate in a 96 well plate using Taqman $\AA$ Universal Mastermix (all of the above from Life Technologies, Foster City, CA).

For data analysis, a $\Delta \mathrm{Rn}$ (normalized reporter value i.e. signal) threshold of 0.1 was chosen based on the linear part of the gene expression curve. Fold changes were calculated using the $\triangle \triangle C T$ method ${ }^{112}$.

For TLR signaling, pre-coated mRNA profiler plates were used (Taqman $\AA$ Array Human Toll Pathway, Core Components, Life Technologies, Foster City, CA). Each of the 48 wells received $3 \mathrm{ng}$ of cDNA (44 wells with gene of interest, 4 of internal controls). Taqman® Universal Mastermix was used to make a reaction volume of $10 \mu \mathrm{L}$ per well. For these experiments, a combination of 18S, GAPDH, and Beta2-Microglobulin were used for internal controls. The StepOne Plus RealTime-PCR-System instrument was used for

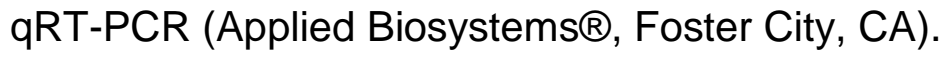




\section{vii.miRNA measurements}

For microRNA screening, Taqman® Microfluidic Low Density Array Cards were used with a total of $1500 \mathrm{ng}$ of diluted RNA per card, divided between 384 wells (Card A, Life Technologies, Foster City, CA). Once samples were loaded (100 $\mu \mathrm{L}$ per port), cards were sealed and centrifuged at $2000 \mathrm{rpm}$ for 2 mins. They were then run using a ViiA ${ }^{\mathrm{TM}} 7$ Real-Time PCR System (Applied Biosystems ${ }^{\circledR}$, Foster City, CA). Four separate wells with RNU6, along with RNU44 and RNU48 were used in combination for an internal control.

For single assay microRNA quantification, specific $5 \mathrm{X}$ primers were used per microRNA for reverse transcription to create CDNA, with specific 20X primers used for qRT-PCR with Taqman® Universal Mastermix using a reaction volume of $10 \mu \mathrm{L}$. The internal control used was RNU6, and all assays were run in duplicate using a 96 well plate using a StepOne Plus RealTime-PCRSystem instrument (Applied Biosystems ${ }^{\circledR}$, Foster City, CA). Like messenger RNA analysis previously described, all microRNA data was analyzed using a cycle threshold of 0.1 using the $\Delta \Delta C T$ method ${ }^{112}$.

\section{viii. Western Blot analysis}

For intracellular protein quantification, monocyte cell culture tubes were centrifuged for a given time point, the cell pellet lysed using RIPA buffer with a 1:1000 concentration of protease and phosphatase inhibitors (Thermo Scientific, Rockford, IL) and stored at $-80^{\circ} \mathrm{C}$ until subsequent analysis. For protein extraction, lysed cells were then sonicated using a Sonifier 250 (Branson Ultrasonics, Danbury, CT). Protein concentrations were quantified using a bicinchoninic acid (BCA) protein quantification assay (Thermo 
Scientific, Rockford, IL) against a standard curve using a SpectraMax Plus384 spectrophotometer at $540 \mathrm{~nm}$.

Protein samples of $30 \mu \mathrm{g}$ were loaded into gradient 4-12\% Bis-Tris Plus 12 well gels in combination with $4 \mathrm{X}$ Bolt ${ }^{\mathrm{TM}}$ LDS Running Buffer with 1:100 2mercaptoethanol. Gels were run in a Bolt ${ }^{\mathrm{TM}}$ Mini Gel Tank in Bolt MES SDS Running Buffer at $180 \mathrm{mV}$ for $45 \mathrm{~min}$ (all from Thermo Scientific, Waltham, MA). Each gel contained two wells loaded with a protein size standard to identify the size of proteins of interest (Protein Western C Standard, Bio Rad, Hercules, CA).

After gel electrophoresis, proteins were transferred to a nitrocellulose membrane using iBlot Gel Transfer Stacks and an iBlot transfer device (Fisher Scientific, Hampton, NH).

Membranes were then blocked with 5\% non-fat dried milk in TBS-T (Tris base 9.68g, sodium chloride $32 \mathrm{~g}$ and Tween-20 $100 \mu \mathrm{L}$ in $100 \mathrm{~mL}$ deionized water adjusted to $\mathrm{pH} 7.6$ ) for $1 \mathrm{~h}$ at room temperature to prevent non-specific binding. Membranes were then incubated overnight with primary antibodies (either rabbit or mouse anti-human) at room temperature. All antibodies were purchased from Cell Signaling (Danvers, MA). Antibody concentrations were as follows; Beta-actin 1:10,000, Vinculin 1:1000, NFkB p65 1:1000, IkB 1:1000, IKK- $\alpha$ 1:1000, IKK- $\beta$ 1:1000, TAK-1 1:1000 diluted in 5\% non-fat dried milk with TBS-T. Phospho-IKK and phospho-TAK1 were diluted at 1:1000 in 5\% Bovine Serum Albumin (BSA) in TBS-T (non-fat dried milk was not used with phosphorylated antibodies to prevent casein interfering with sites of phosphorylation). After incubation with the primary antibody, membranes were 
washed three times for $5 \mathrm{~min}$ in TBS-T before incubation with secondary antibodies (goat anti-rabbit or horse anti-mouse as appropriate) at a concentration of $1: 1000$ for $1 \mathrm{~h}$ at room temperature.

After a further three washes as above, membranes were finally incubated in Pierce ECL Substrate for 5 min (Thermo Scientific, Waltham, MA). Finally, the membrane was imaged using the ChemiDoc MP system (Bio Rad, Hercules, $\mathrm{CA}$, courtesy of Dr. Jason Smith) and protein densitometries measured using ImageLab software (Bio Rad, Hercules, CA). For each protein of interest, relative density units were expressed as a ratio over an internal loading control from the same sample (either Beta Actin or Vinculin depending on the molecular weight of the protein).

\section{ix. Inhibition of IKK}

To determine the direct role of IKK in monocyte function and dysfunction, a small molecule specific inhibitor of IkK called IkK-16 was purchased (SigmaAldrich, St. Louis, MO). It was prepared in accordance with manufacturer's instructions. In brief, powder preparation was reconstituted in $180 \mu \mathrm{L}$ of dimethyl sulfoxide (DMSO) and aliquoted in stock $10 \mu \mathrm{L}$ ampules and stored at $-80^{\circ} \mathrm{C}$. For each experiment, IKK-16 was prepared fresh by a $1: 50$ dilution with DMSO from stock and then diluted in 1:1000 with media (as described under Monocyte Isolation) for use. Doses of IKK-16 were given at $100 \mathrm{nM}$ unless otherwise specified. For a $100 \mathrm{nM}$ dose, $100 \mu \mathrm{L}$ of IKK-16 was given per $\mathrm{mL}$ of cultured cells. Preliminary experiments demonstrated that DMSO had no effect on monocyte function or viability at such a substantial dilution. 
Loss of function of IKK phosphorylation is confirmed in the Results section of Specific Aim 2 (see Figure 29).

\section{x. Statistical analysis}

Data are shown as mean \pm standard error of the mean. For a given condition, each donor was compared within the same individual. Therefore, in general paired, related analyses were used. For individual comparisons, a two-tailed paired t-test or Wilcoxon signed rank were used. Statistical analyses were performed using SPSS version 21 (PASW Statistics, IBM, Chicago, IL). Significance was determined with an alpha of 0.05 . Sample size calculations, power analyses and more complex statistical analyses are described in the relevant sections. 


\section{CHAPTER V \\ DEVELOPING A MODEL OF MONOCYTE IMPAIRMENT}

INTRODUCTION

Monocyte impairment is estimated to occur in approximately 1 in 6 patients. It is not clear whether these patients have a genetic predisposition limiting their cellular responsiveness, or whether this is acquired from the insult itself. Therefore, predicting which patients will have monocyte impairment is challenging. The study of mechanisms and modulation of monocyte impairment in surgical patients is complex and expensive. There are a large number of additional factors which may influence these measurements such as co-morbidity and the effect of interventions such as surgery, antimicrobial drugs and adjunctive drugs such as heparin and fluid therapy. Therefore, study of the human immunological response to a major insult in a consistent, reproducible way requires an alternative solution.

Sepsis models in animals, such as mice, have been used to study monocyte impairment in a variety of approaches. This often involves intraperitoneal delivery of endotoxin, before either a further in-vivo challenge or isolation of peritoneal macrophage or bone marrow derived cells for ex-vivo LPS stimulation. The major limitation with this approach is whether the findings can be extended to a human population. This was eloquently discussed in the letter "Mice are not men" by the Glue Grant group in PNAS, 
$2013^{113}$. Cell lines have the benefit of being of human origin and are reproducible, however, do not offer the genetic variability that exists in the human population.

In this model, we use monocytes from healthy volunteers to preserve the variability from person to person, that are impaired equally in standardized fashion by an initial low dose of LPS $(10 \mathrm{ng} / \mathrm{mL})$. The goal was to produce a model where the cells with previous exposure to LPS have a reproducible $d$ ecrease in their capacity to produce TNF- $\alpha$ in response to a subsequent challenge. TNF- $\alpha$ is the most defined and accepted marker that defines a blunted monocyte response in high quality immunological studies. However, IL-10 and HLA-DR expression represent important functions of the monocyte which also bear clinical correlation and therefore were also measured.

\section{RESULTS}

\section{Isolated Monocyte Culture - Cell Purity and Viability}

Prior to isolation, leukocytes in whole blood can be seen in three broad categories by flow cytometry which are neutrophils (polymorphonuclear cells), lymphocytes and monocytes (circled, Figure 12A). Following CD14 magnetic bead isolation, a single cell population can be observed (Figure 12B). A small amount of debris can be seen in the bottom left hand corner of this scatterplot, which is normal for cells after culture and multiple centrifugations. By gating on the monocyte population (as per the circle in Figure 12B), consistently over $95 \%$ these cells are highly positive for the monocyte markers CD14 (FITC) and HLA-DR (PE). A representative scatterplot is shown in Figure 12C. This confirms a pure single cell monocyte population for study. 
Figure 12. Monocyte purity following CD14 magnetic positive selection technique from whole blood.
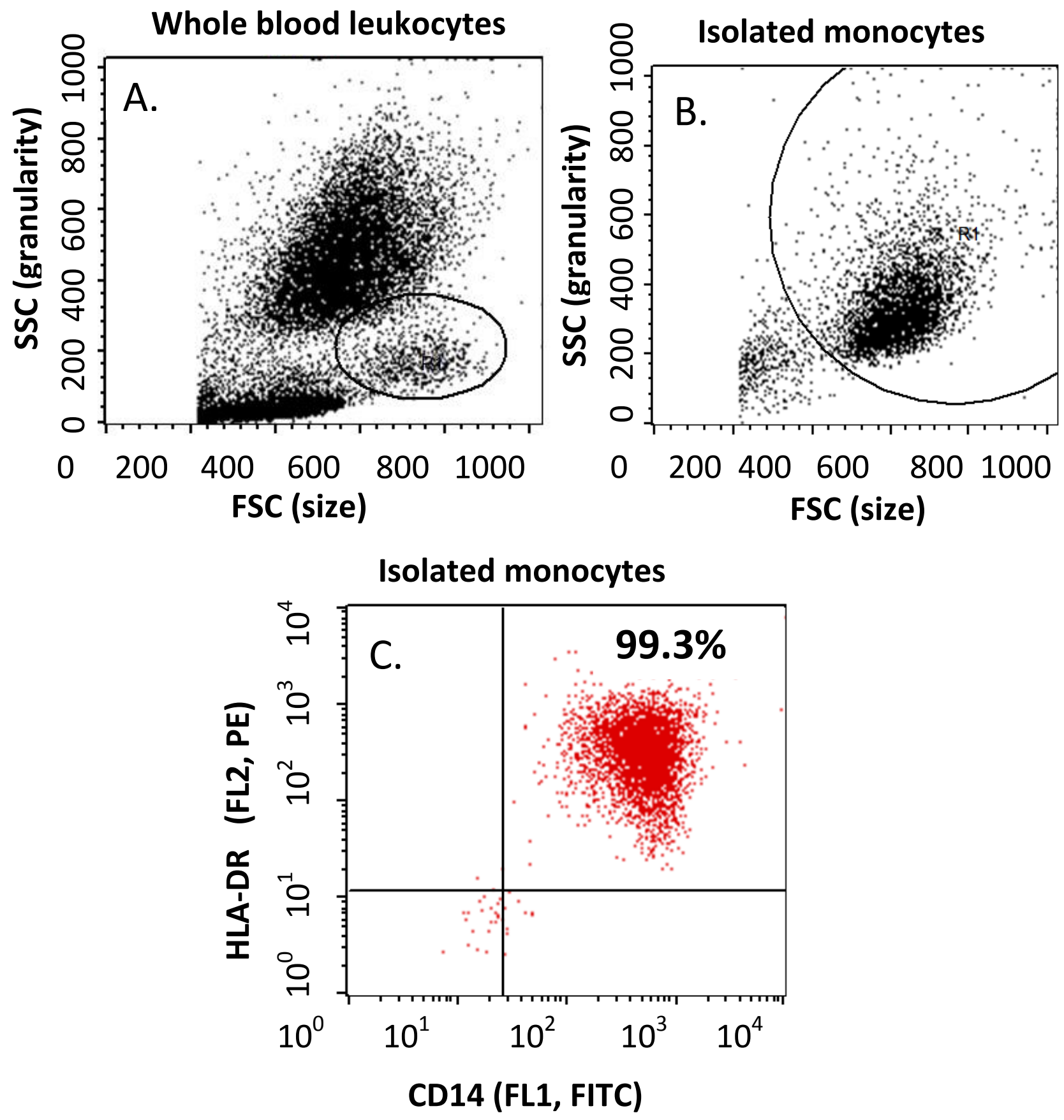


\section{Legend for Figure 12.}

The distribution of monocytes in a three-part differential of leukocytes in whole blood prior to monocyte isolation is shown in $(\boldsymbol{A})$, based on its size (forward scatter, FSC) and granularity (side scatter, SSC) properties. Following isolation by magnetic bead positive selection technique, a single cell population is identified in the gate circled (B). Material in the lower left hand corner represents debris, normal for flow cytometry of such cells. Of the cells isolated, 99.3\% of gated cells are CD14 (FL1) and HLA-DR (FL2) positive demonstrating a pure, viable monocyte population $(\boldsymbol{C})$. This representative sample is taken 33 hrs after isolation. 
Figure 13. Time course experiment of monocyte viability in cell culture.

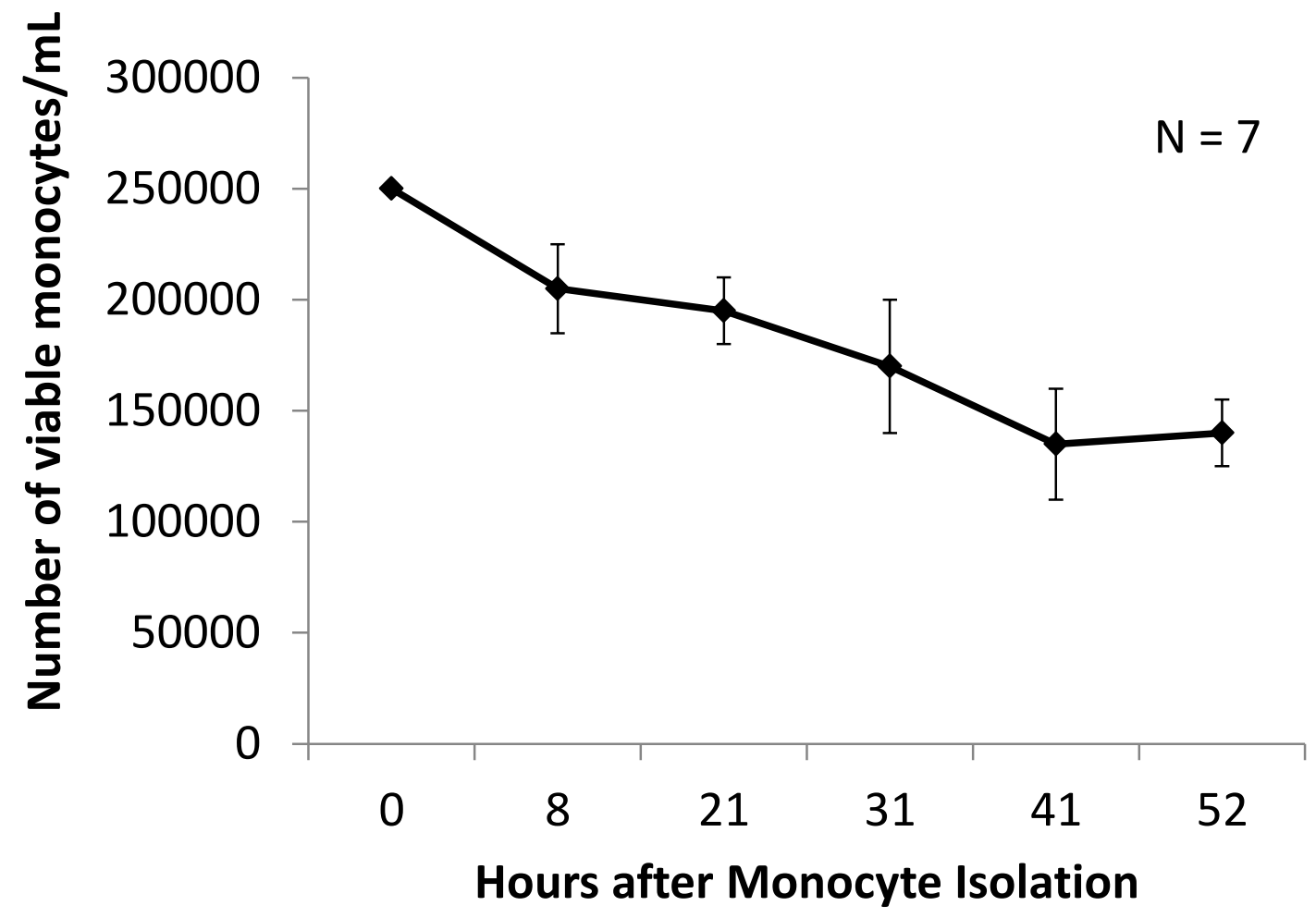

The viability of isolated monocytes were determined by culturing cells over time and number of viable monocytes manually counted by standard microscopy after Trypan blue staining. By 24 hours, cell loss is approximately a third, reaching about half of the initial number of cells by 48 hours. $N=7$. 
Figure 14. Experimental design of the impaired monocyte model.

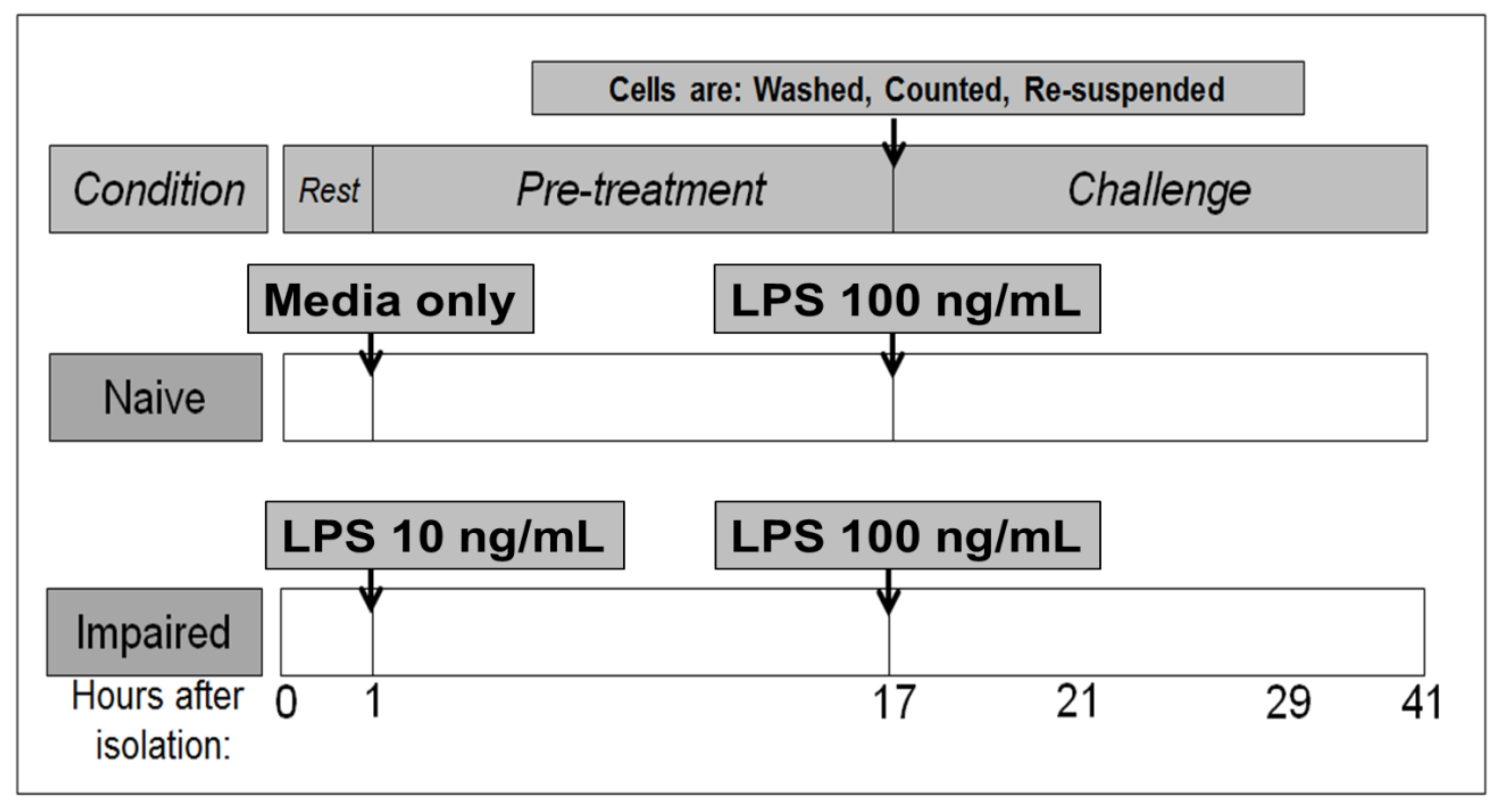

After isolation of monocytes from healthy volunteers, cells are cultured and divided into two conditions; naïve and impaired. After $1 \mathrm{~h}$ of rest, the naïve cells remain in media only until $17 \mathrm{~h}$. By comparison, low dose $10 \mathrm{ng} / \mathrm{mL}$ is added to the impaired cell culture until $17 \mathrm{~h}$. At $17 \mathrm{~h}$, tubes of cell suspension are centrifuged, and cells are washed and counted by microscopy and resuspended to $0.5 \times 10^{6} / \mathrm{mL}$ concentration to ensure equal cell concentrations across both naïve and impaired conditions. Cells are then challenged with $100 \mathrm{ng} / \mathrm{mL}$ LPS to determine monocyte responsiveness at various time points indicated. 
The viability of these cells in culture were studied over time, demonstrated in Figure 13. It should be noted that there is a relatively consistent loss of monocytes of approximately a third by 24 hours, reaching approximately a half of cells by 48 hours after cell isolation.

Experimental design of the impaired monocyte model is described in detail in the Methods section, but is summarized in Figure 14.

\section{TNF- $\alpha$ production}

The naïve cells in standard media without initial low dose LPS stimulation secreted approximately $1500 \mathrm{pg} / \mathrm{mL}$ TNF- $\alpha$ by 17 hours (Figure 15). Low dose LPS $10 \mathrm{ng} / \mathrm{mL}$ stimulated a trend towards increased TNF- $\alpha$ levels by 17 hours. After cells were washed and resuspended, both conditions were cultured with a $100 \mathrm{ng} / \mathrm{mL}$ LPS challenge. In general, the TNF-a levels in both conditions were lower at time points after 17 hours compared with those at the end of the initial 17 hour interval. Critically, the naïve cells (illustrated in green) were able to produce a consistently higher level of TNF- $\alpha$ after the $100 \mathrm{ng} / \mathrm{mL}$ LPS challenge, compared to the impaired cells which demonstrated sustained suppression of TNF- $\alpha$ production in response to such an $100 \mathrm{ng} / \mathrm{mL}$ LPS challenge (seen maximally at 4 and 12 hours after the $100 \mathrm{ng} / \mathrm{mL}$ LPS challenge).

\section{IL-10 production}

In contrast to TNF- $\alpha$, IL-10 levels in general increased over time in culture for both conditions (Figure 16). In response to the initial $10 \mathrm{ng} / \mathrm{mL}$ LPS, IL-10 trended to a lower level in the impaired cells compared to the naïve cells at 17 
hours, without reaching statistical significance. However, in response to the $100 \mathrm{ng} / \mathrm{mL}$ LPS challenge, the capacity for monocytes to produce IL-10 was consistently suppressed in the impaired cells at 4, 12 and 24 hours after the $100 \mathrm{ng} / \mathrm{mL}$ LPS challenge.

\section{HLA-DR expression}

In both conditions, HLA-DR expression increased over the first 17 hours from baseline levels after isolation. Low dose $10 \mathrm{ng} / \mathrm{mL}$ LPS caused a small but consistent decrease in HLA-DR expression compared to the naïve cells (Figure 17). This equated to impaired cells expressing approximately only 5$15 \%$ lower levels of HLA-DR compared to the naïve cells; however there was a considerable amount of variability from donor to donor. There were no differences in HLA-DR expression between conditions for the remaining time points. 
Figure 15. TNF- $\alpha$ production in the impaired monocyte.

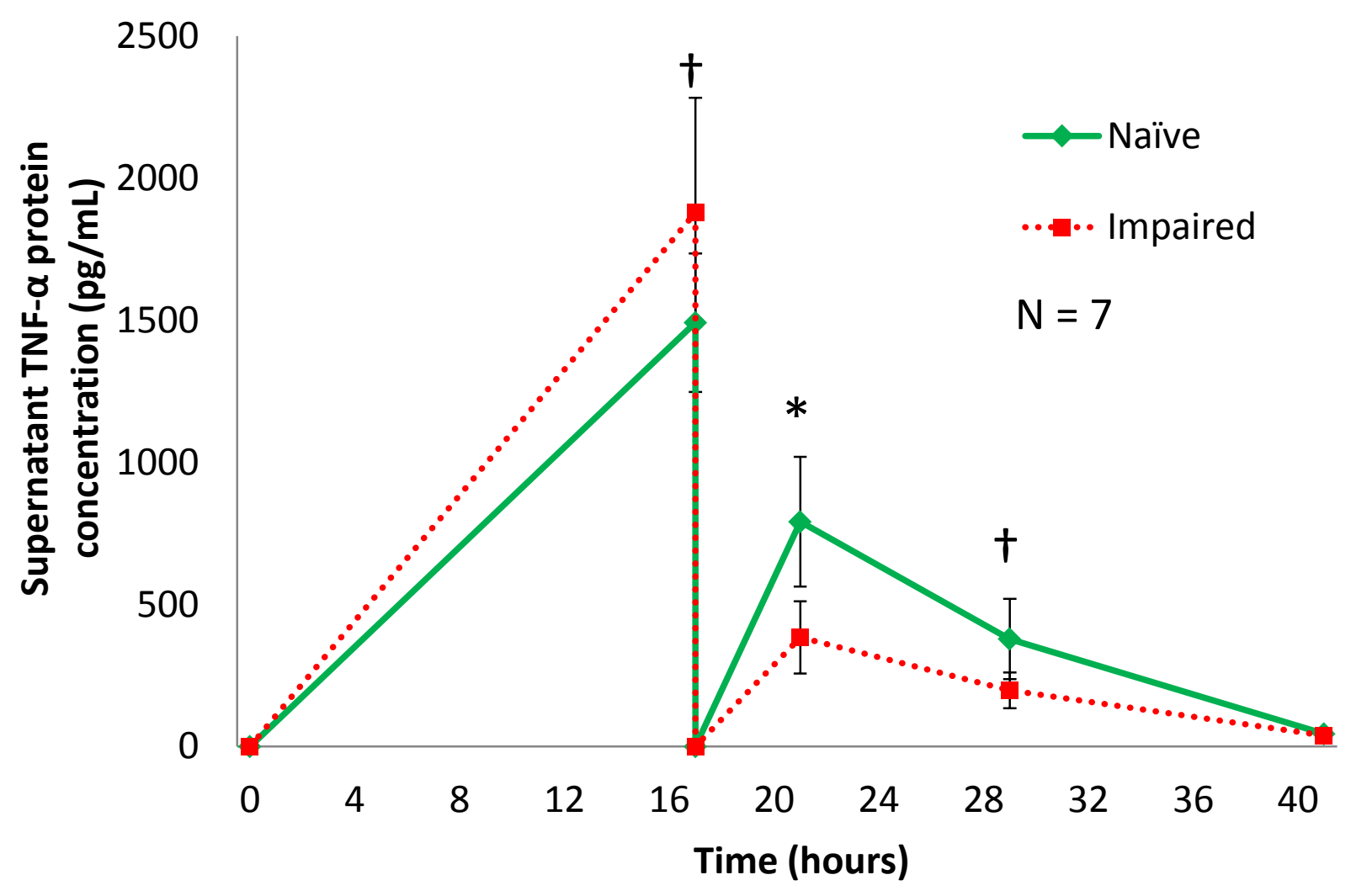

Supernatant protein concentration of TNF- $\alpha$, a pro-inflammatory cytokine, was measured at various time points by ELISA to compare naïve cells (green) against impaired cells (red). Low dose LPS $10 \mathrm{ng} / \mathrm{mL}$ at $1 \mathrm{~h}$ leads to a modest increase in TNF- $\alpha$ production by $17 \mathrm{~h}$ in the impaired cells. However, in response to a high dose $100 \mathrm{ng} / \mathrm{mL} L P S$ challenge given at $17 \mathrm{~h}$, the same impaired cells have a sustained suppression of TNF-a production thereafter when compared to the naïve cells. Mean \pm SEM. $N=7 .{ }^{*} p<0.05, \dagger p=0.07$. Wilcoxon signed rank test. 
Figure 16. IL-10 production in the impaired monocyte.

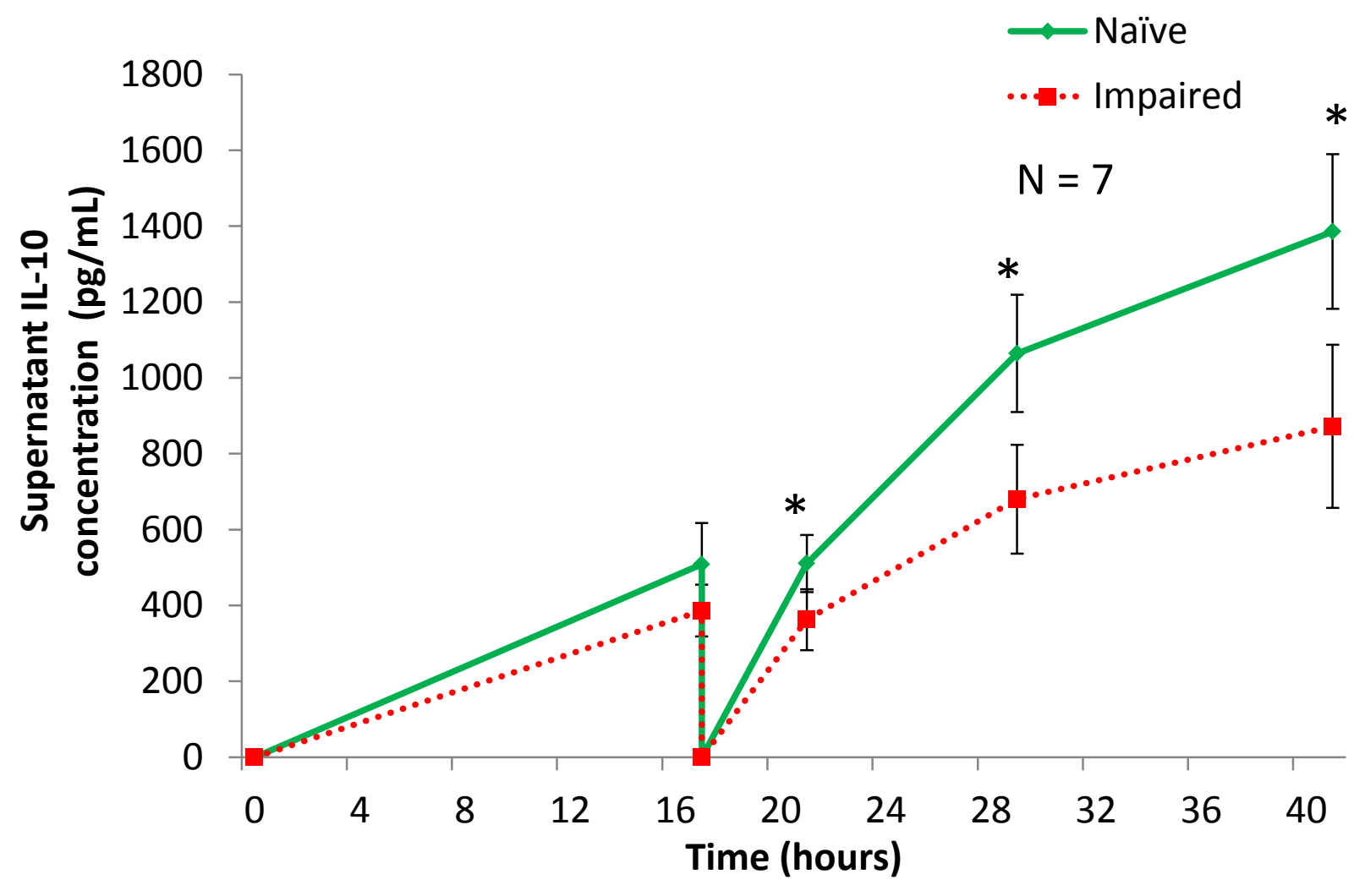

The supernatant protein concentration of IL-10, an anti-inflammatory cytokine, was measured at various time points by ELISA to compare naïve cells (green) against impaired cells (red). Low dose $10 \mathrm{ng} / \mathrm{mL}$ LPS at $1 \mathrm{~h}$ leads to a minor but not significant decrease in IL-10 concentration by $17 \mathrm{~h}$ in the impaired cells. However, in response to a high dose $100 \mathrm{ng} / \mathrm{mL}$ LPS challenge given at $17 \mathrm{~h}$, impaired cells have a sustained suppression of IL-10 production when compared to the naïve cells. Mean $\pm S E M . N=7 .{ }^{*} p<0.05$, Wilcoxon signed rank test. 


\section{IL-6 production}

There was a slight trend of low dose $10 \mathrm{ng} / \mathrm{mL}$ LPS increasing IL-6 production by 17 hours compared to naïve cells, but this did not reach statistical significance. Subsequent to the $100 \mathrm{ng} / \mathrm{mL}$ LPS challenge, the impaired cells had a sustained decrease in the ability to produce IL-6 when compared to the naïve cells (Figure 18). This was most evident at 29 hours and 41 hours (corresponding to 12 hours and 24 hours after the $100 \mathrm{ng} / \mathrm{mL}$ LPS challenge).

\section{TNF- $\alpha$ gene expression (messenger RNA)}

Figure 19 demonstrates differences in intracellular TNF- $\alpha$ messenger RNA levels relative to the naïve monocyte, as measured by qRT-PCR. Low dose $10 \mathrm{ng} / \mathrm{mL}$ LPS in the impaired cells leads to a modest increase in TNF- $\alpha$ gene expression seen at 2, 4 and 8 hours after isolation. This difference begins to correspond to similar levels in the naïve monocyte by 17 hours. In response to the $100 \mathrm{ng} / \mathrm{mL}$ LPS challenge, however, TNF- $\alpha$ gene expression is decreased relative to the naïve monocyte. This difference is seen maximally at 19 hours (2h after the $100 \mathrm{ng} / \mathrm{mL}$ LPS challenge). 
Figure 17. HLA-DR expression in the impaired monocyte.
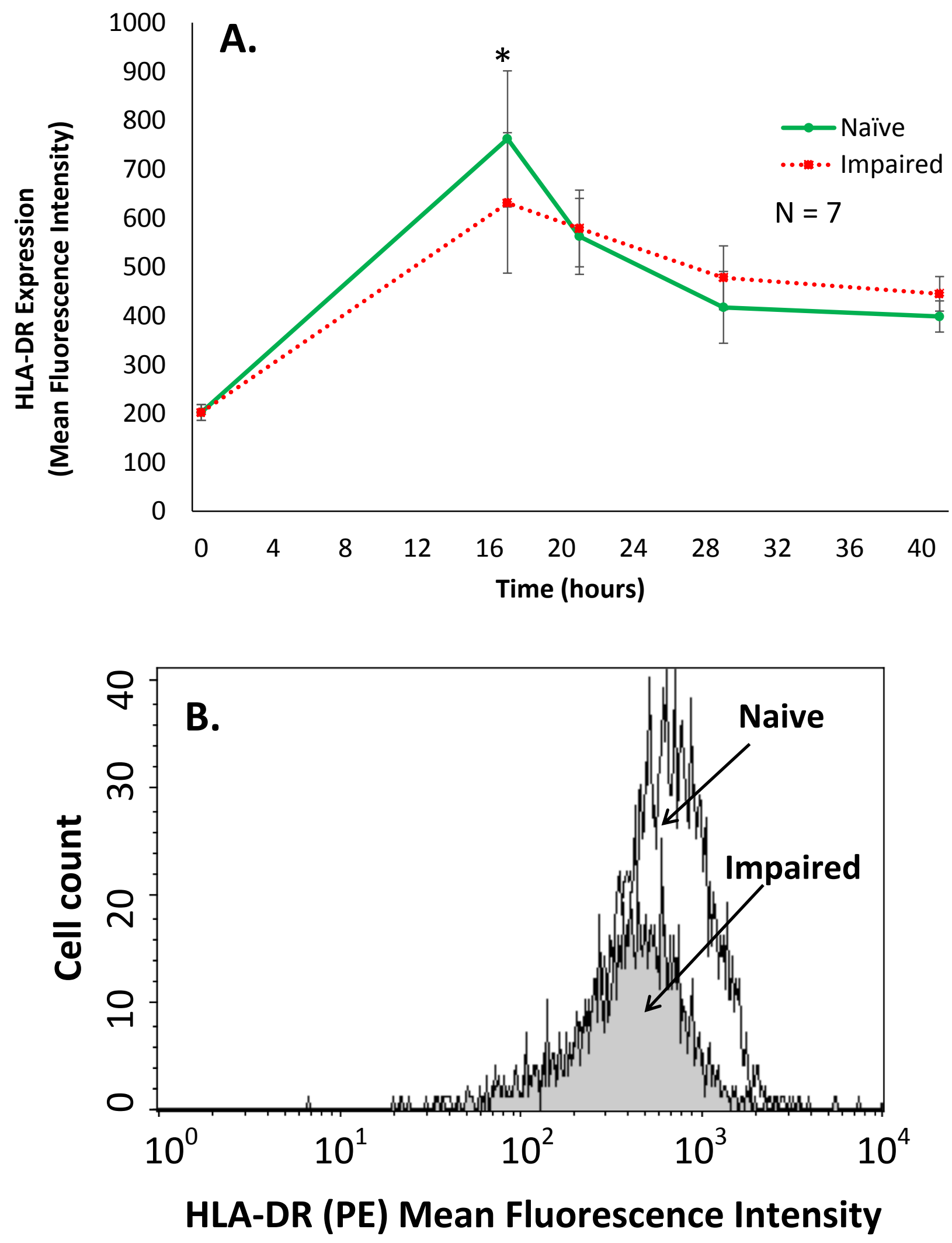


\section{Legend for Figure 17.}

Monocyte HLA-DR, a MHC I/ class surface marker critical for the function of antigen presentation, was determined by flow cytometry at various time points (A). Mean Fluorescence Intensity of HLA-DR was measured comparing naïve cells (green) against impaired cells (red). Low dose $10 \mathrm{ng} / \mathrm{mL} L P S$ at $1 \mathrm{~h}$ led to a small but consistent decrease in HLA-DR expression at $17 \mathrm{~h}$ in the impaired cells. When both conditions were treated with a high dose 100 $n g / m L ~ L P S$ challenge at $17 \mathrm{~h}$, no significant differences in HLA-DR expression were observed between the naïve and impaired monocytes. Mean \pm SEM. $N$ $=7 .{ }^{*} p<0.05$, Wilcoxon signed rank test.

A histogram of HLA-DR expression in monocytes both naïve cells (white) and impaired cells (gray) at $17 \mathrm{~h}$ is shown in (B). This is a representative sample qualitatively demonstrating the decreased HLA-DR expression in impaired conditions. 
Figure 18. IL-6 production in the impaired monocyte.

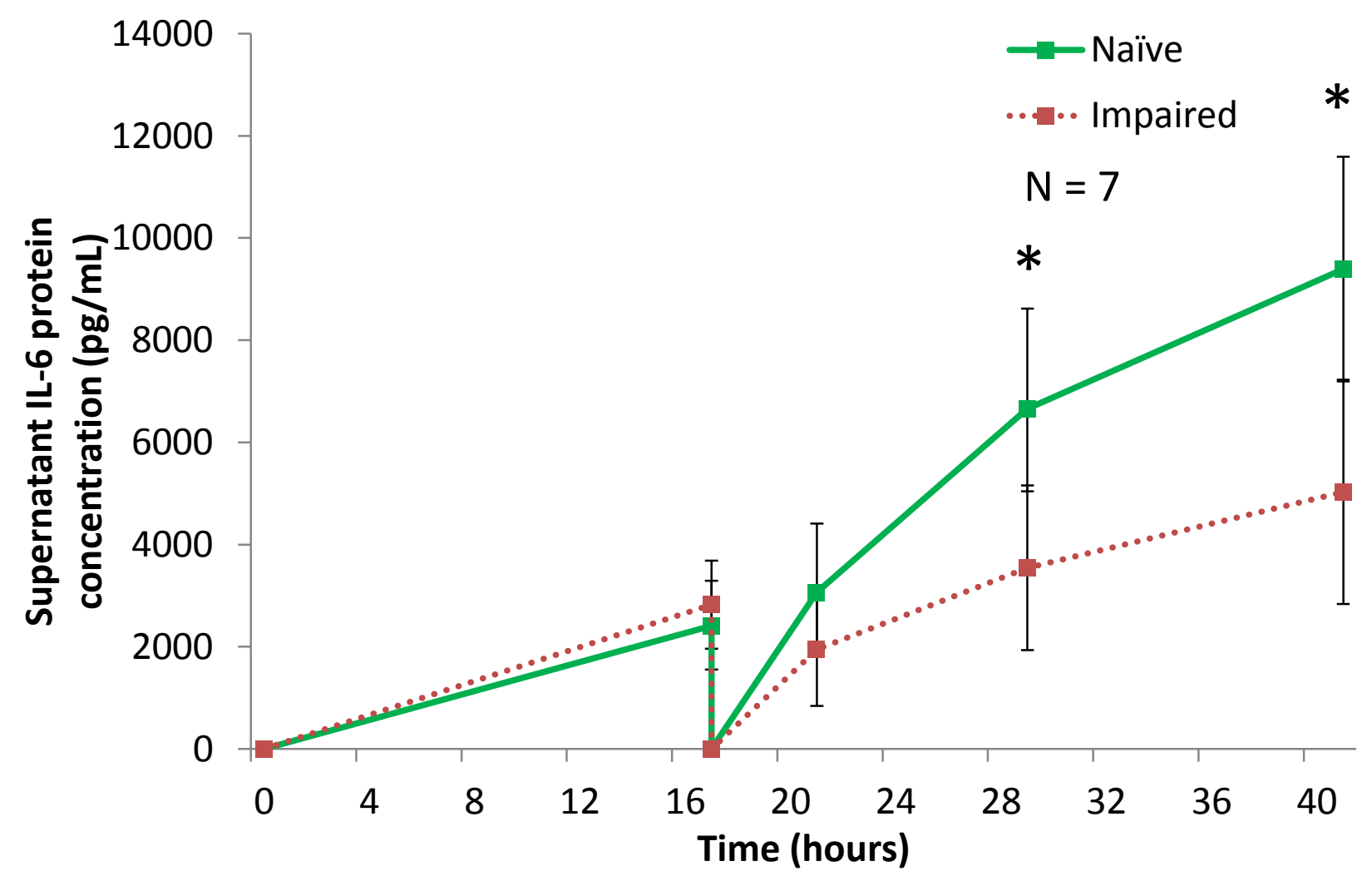

IL-6 supernatant protein concentration was measured at various time points by ELISA, comparing naïve cells (green) against impaired cells (red). Low dose $10 \mathrm{ng} / \mathrm{mL}$ LPS given at $1 \mathrm{~h}$ led to a minor but not significant increase in IL-6 production by $17 \mathrm{~h}$ in the impaired cells. However, in response to a high dose $100 \mathrm{ng} / \mathrm{mL}$ LPS challenge given at $17 \mathrm{~h}$, impaired cells appear to have a sustained suppression of IL-6 production when compared to the naïve cells. Mean \pm SEM. $N=7 .{ }^{*} p<0.05$, Wilcoxon signed rank test. 
Figure 19. TNF- $\alpha$ gene expression (mRNA) in the impaired monocyte.
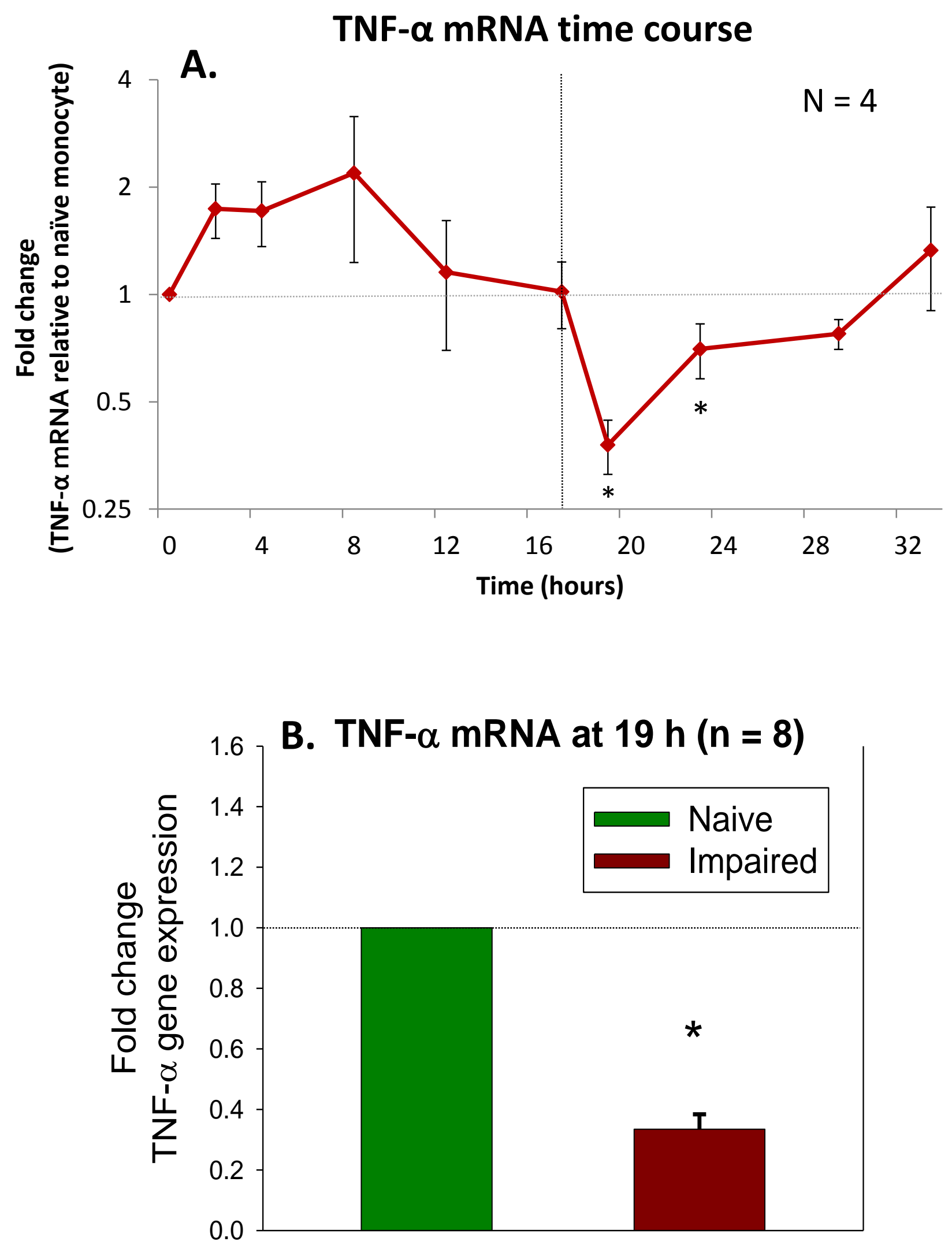


\section{Legend to Figure 19.}

Intracellular TNF- $\alpha$ gene expression was examined in the naïve and impaired monocyte by qRT-PCR. A time course experiment is shown in the top graph using 4 donors (A). Low dose $10 \mathrm{ng} / \mathrm{mL}$ LPS upregulated TNF- $\alpha$ mRNA expression rapidly over the first 4 to 8 hours after treatment, then began "turning off" by $17 \mathrm{~h}$. When both conditions were incubated with high dose 100 $\mathrm{ng} / \mathrm{mL}$ LPS challenge at $17 \mathrm{~h}$, the impaired cells demonstrated a decreased level of mRNA expression relative to naïve cells. This genomic response early after LPS stimulation corresponds with the sustained differences seen in TNFa protein (shown earlier in Figure 15). The maximal level of suppression of $T N F-\alpha$ gene expression in the impaired monocyte is seen at $19 \mathrm{~h}$, relative to the naïve monocyte ( $2 h$ after the $100 \mathrm{ng} / \mathrm{mL} L P S$ challenge). $N=4$.

The bottom figure represents qRT-PCR experiments focused at this $19 \mathrm{~h}$ time point (B). Impaired monocytes (red) have a profound downregulation of TNF- $\alpha$ $m R N A$ relative to naïve cells. $N=8$. Mean $\pm S E M .{ }^{*} p<0.05$, Wilcoxon signed rank test. 


\section{DISCUSSION}

Monocytes have various critical immunological functions in orchestrating a well-balanced response to an insult. Cytokine production is a highly regulated set of small functional proteins allowing activation and differentiation of leukocytes governing the immune response. TNF- $\alpha$ and IL-6 represent proinflammatory cytokines. These are produced early after a stimulus and activate the innate and adaptive system to in turn produce further cytokines, hence the term "cytokine cascade". This pattern is demonstrated by our data, where TNF- $\alpha$ and IL-6 are produced early and trend to an early increase in the $10 \mathrm{ng} / \mathrm{mL}$ LPS treated cells.

TNF- $\alpha$ is secreted primarily from monocytes and macrophages often within 30 min of an inciting event. As part of its autocrine function, it promotes the activation and differentiation of macrophages, prolongs their survival and increases their production from progenitor cells. Direct injection of TNF- $\alpha$ into animals produces a shock-like state, which is also associated with increased vascular permeability, pulmonary edema and activation of the coagulation system. TNF- $\alpha$ has been described as the "master regulator" of inflammatory cytokine production, but the blockade of TNF- $\alpha$ has been shown to improve mortality in animal models ${ }^{114}$.

The suppression of TNF- $\alpha$ in response to a second LPS exposure has defined models of impaired monocyte function, or "endotoxin tolerance", throughout immunology literature ${ }^{5,68,69}$. In our results utilizing this type of model, there is a consistent depression of TNF- $\alpha$ production in cells with prior LPS exposure, thus validating our model. This is in keeping with many other 
groups ${ }^{7}$. Furthermore, the changes in TNF- $\alpha$ gene expression prove that the suppressed response occurs at a level of intra-cellular genomic signaling which is clearly different between the two conditions. These time course experiments demonstrate that increased TNF- $\alpha$ observed in response to 10 $\mathrm{ng} / \mathrm{mL}$ LPS is produced from newly synthesiszed messenger RNA, therefore true production in response to a stimulus, rather than pre-formed TNF- $\alpha$ released in impaired conditions compared with naïve monocyte. Contrast the changes in TNF- $\alpha$ gene expression seen in Figure 19A to the clinical scenario in Figure 1. We believe these similarities demonstrate the clinical relevance of our model.

IL-10 is a centrally important anti-inflammatory cytokine which has an important role in counterbalancing the pro-inflammatory response. In excessive amounts, IL-10 can lead to monocyte deactivation, with high levels related to the magnitude of injury. Our results show that IL-10 production, representative of the anti-inflammatory response, is also impaired in cells treated with prior $10 \mathrm{ng} / \mathrm{mL}$ LPS. This is in agreement with some groups ${ }^{7,66}$, although various in-vivo studies demonstrate higher IL-10 levels ${ }^{68,115}$. This difference may be related to the interaction between monocytes and T-cells which is not preserved in our model of single cell suspension.

Monocyte HLA-DR expression is reported less often in the literature than TNF- $\alpha$ in this type of experimental model. Our results show a decrease in HLA-DR expression in response to low dose $10 \mathrm{ng} / \mathrm{mL}$ LPS, however this difference is small and not seen at later time points by which both cellular conditions have received LPS exposure. The magnitude of the HLA-DR downregulation is less than expected, and may be that the small effect of low 
dose $10 \mathrm{ng} / \mathrm{mL}$ LPS in the context of cell activation by the process of isolation and ex-vivo cell culture, as demonstrated by the increased size and granularity properties of the monocytes compared to those seen in whole blood prior (pre-isolation) (Figure 12A \& B), and the higher than expected levels of TNF-a in the naïve cells by 17 hours (Figure 15). This decrease in HLA-DR expression in the impaired cells is however consistent and broadly in keeping with literature findings ${ }^{67,68}$.

Elevated levels of IL-6 have long been associated with mortality in trauma and sepsis. IL-6 is a potent inducer of the acute phase response, with pleiotropic effects on proliferation, differentiation and maturation of progenitor cells as well as controlling cellular metabolic activities. These results demonstrate that impaired monocytes have a decreased capacity to produce IL-6 production in response to an LPS challenge. This is in keeping with previous literature ${ }^{7,66,68}$.

There were numerous difficulties in developing this model. Different rest periods after isolation were evaluated, including no rest, 1 hour and 8 hours of rest. LPS doses of $2 \mathrm{ng} / \mathrm{mL}, 10 \mathrm{ng} / \mathrm{mL}$ and $100 \mathrm{ng} / \mathrm{mL}$ were tested as the initial stimulus. Both 16 and 24 hours were used for the initial duration of LPS exposure, and various monocyte cell concentrations were used. We found that after 1 hour of rest after isolation, followed by an initial dose of 10 $\mathrm{ng} / \mathrm{mL}$ LPS for 16 hours, led to the most consistent impairment of monocyte function in response to a subsequent $100 \mathrm{ng} / \mathrm{mL}$ LPS challenge (Figure 14).

Therefore, the model appears to reproducibly produce monocytes which have impaired functions of pro- and anti-inflammatory cytokine 
production as well as decreased antigen-presenting capacity, as measured by HLA-DR expression. This model therefore allows for further study of the underlying immunological mechanisms of impaired monocyte function, an important factor in the outcome of surgical patients at risk of infection (Figure 20). 
Figure 20. Calculating host defense liabilities as a causative factor in the probability of infection in the surgical patient.

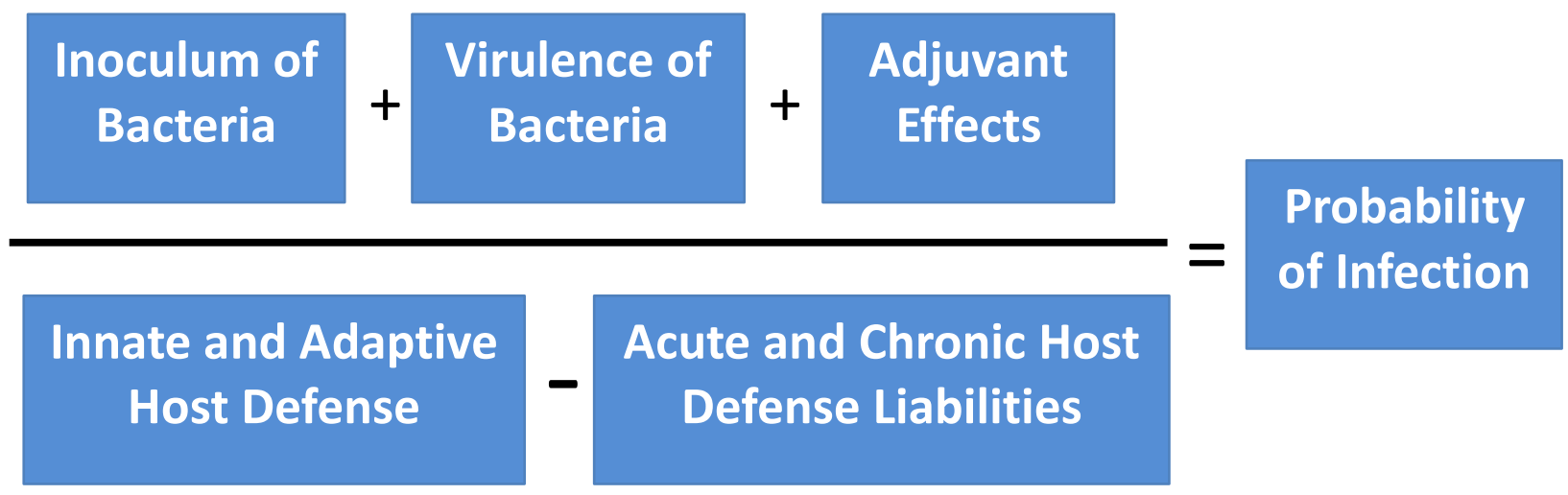

There are multiple and often unpredictable factors relating to whether a surgical patient will be susceptible to nosocomial infection. This model of impaired monocyte function allows for the study of the 'Innate and Adaptive Host Defenses', and it's 'Acute Host Defense Liabilities' in a reproducible fashion.

Adapted from Fry DE, Surgical Infection Society Annual Meeting 2016, FL. 


\section{CHAPTER VI \\ IKK SIGNALING IN THE IMPAIRED MONOCYTE \\ INTRODUCTION}

The knowledge and understanding of molecular mechanisms governing the immune response has improved considerably over the last 20 years. Various dynamic and complex inflammatory pathways, such as NFKB, respond to stimuli, but also have negative feedback regulation to counteract the proinflammatory response. If these are inadequate, persistent inflammation can be harmful to the patient and can lead to multi-organ failure and death. By comparison, an exaggerated compensatory anti-inflammatory response can predominate which can lead to an impaired host response and insufficient pathogen clearance.

The previous chapter demonstrated a decrease in the monocyte gene expression of TNF- $\alpha$ in response to a subsequent LPS challenge. This suggests that there may be a difference in NFKB signaling between naïve and impaired conditions, as this is one of the critical mechanisms of proinflammatory signaling. Activation of NFKB signaling occurs in response to TLR ligands like LPS, with a cascade of phosphorylation from its upstream kinase, Inhibitor of KB Kinase (IKK). Subsequently, the release of the "brakes" in the pathway, IKB, occurs which allows translocation of the active subunit p65 to the nucleus to activate pro-inflammatory genes such as TNF- $\alpha$. Therefore, if this pathway is suppressed, this would account for the decreased monocyte responsiveness seen both in the impaired monocyte model and in the sick patient. Interestingly, various negative regulators are indeed 
downstream from NFKB and therefore when this pathway is activated, both the pro-inflammatory cytokines are synthesized but also the slower acting compensatory anti-inflammatory molecules are released.

The aim of this chapter is to first determine if there are alterations in NFKB signaling between the naïve and impaired conditions. If this was the case, we then could undertake a detailed study of IKK, upstream of NFKB, in terms of the total concentration and levels of activation in the impaired monocyte. This could then help elucidate the mechanisms underpinning a possible cause and effect relationship between IKK suppression and the diminished inflammatory response in the impaired monocyte.

\section{RESULTS}

\section{NFKB signaling in the impaired monocyte}

Monocytes cultured in low dose $10 \mathrm{ng} / \mathrm{mL}$ LPS for 16 hours were challenged with an $100 \mathrm{ng} / \mathrm{mL}$ LPS challenge for 60 minutes. At this time point, impaired monocytes, which had demonstrated an impaired response in the previous chapter, had a lower protein concentration of p65, the active subunit of NFKB, compared to naïve cells (Figure 21 A \& B). In addition, a representative histogram using phosflow technology demonstrated that these impaired cells have a decrease in the level of phosphorylation at 30 minutes after the 100 $\mathrm{ng} / \mathrm{mL}$ LPS challenge, compared to naïve monocytes (Figure 21C).

Levels of IKB, the "brakes" in the NFKB pathway, were similar at 17 hours. However, naïve cells achieved effective IkB degradation with low levels 
by 60 minutes after the $100 \mathrm{ng} / \mathrm{mL}$ LPS challenge (Figure 22B), compared to the higher IKB levels in impaired cells (i.e. less effective degradation). 
Figure 21. NFKB (p65) signaling in the impaired monocyte.

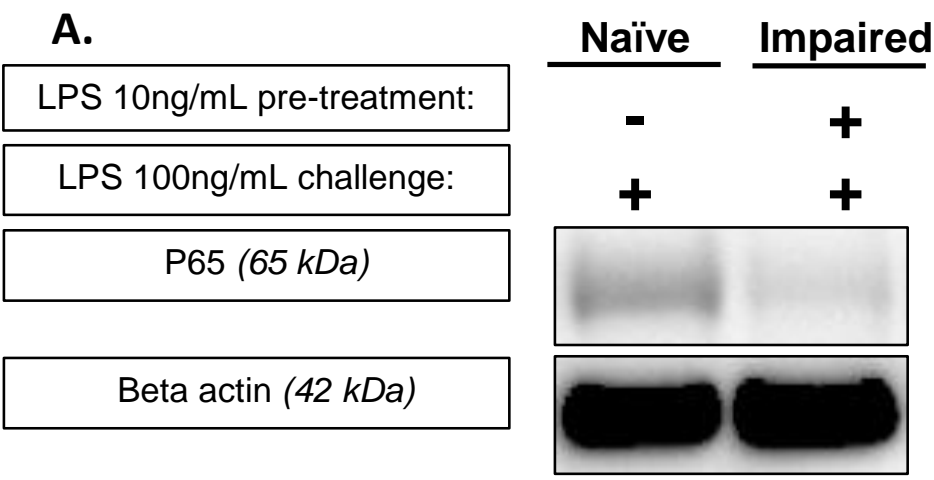

B.

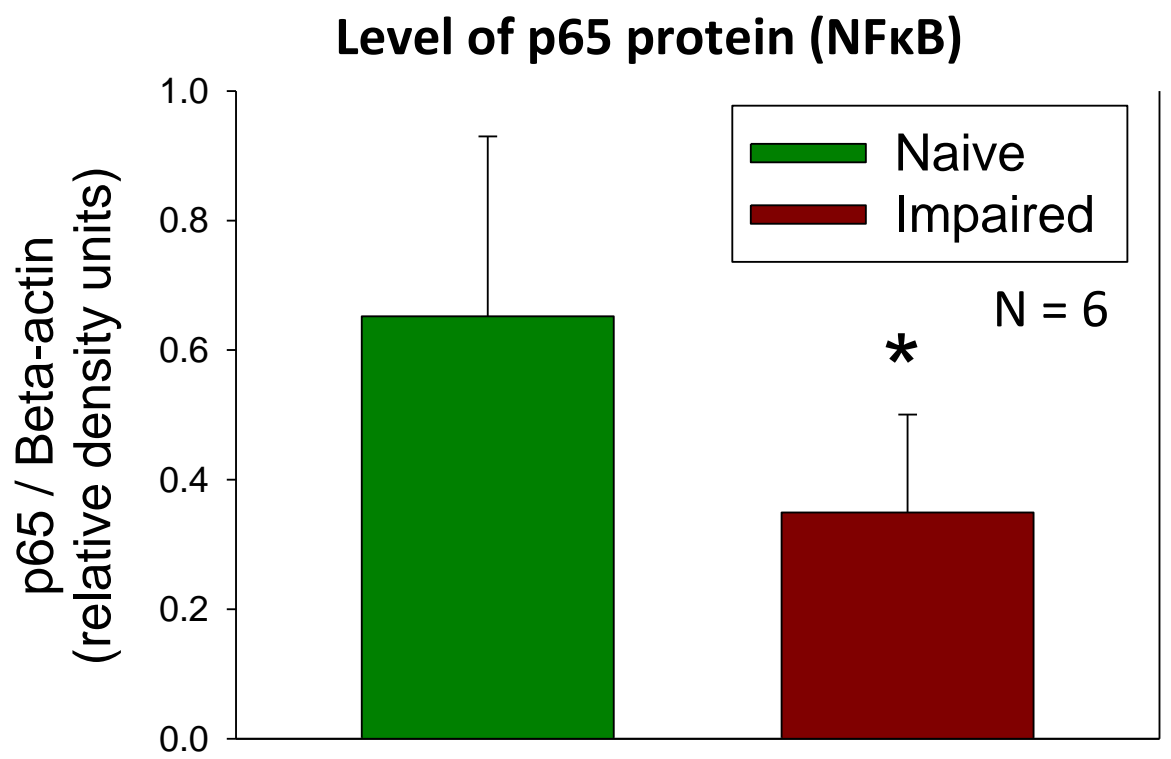

p65 (NFKB) phosphorylation

C.

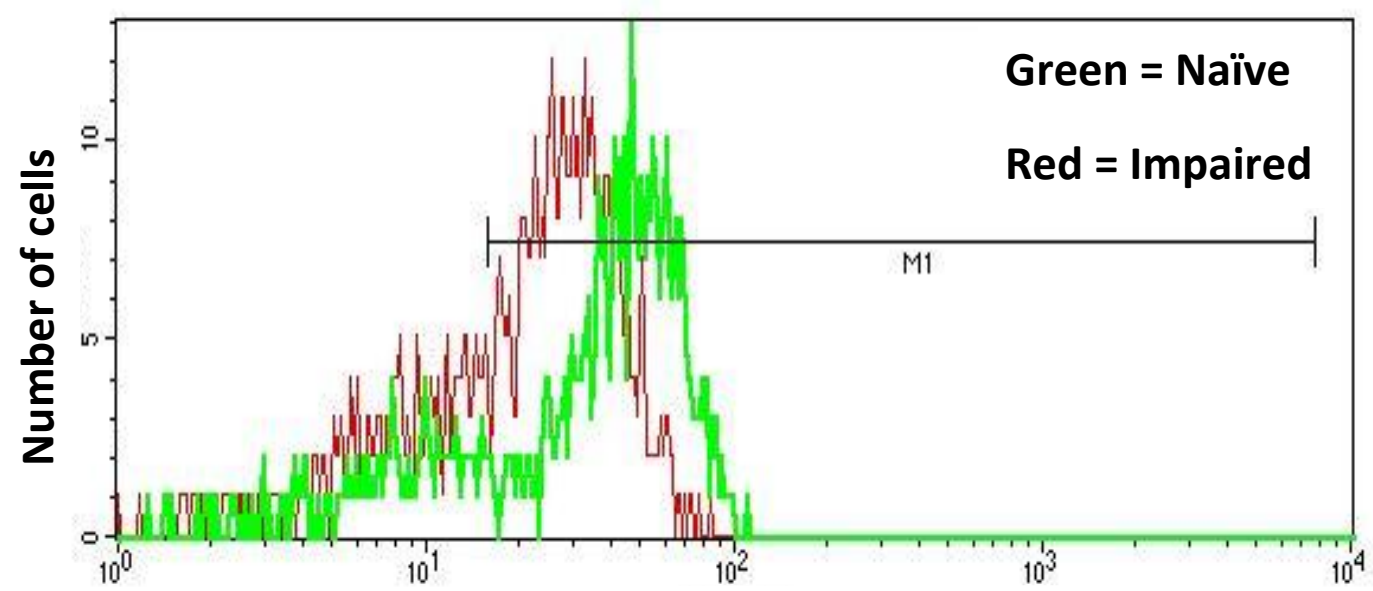

FL4 (phospho-p65) 


\section{Legend to Figure 21.}

Monocytes were cultured either in naïve (media only) or impaired (low dose $10 \mathrm{ng} / \mathrm{mL} L P S$ ) conditions for $16 \mathrm{~h}$. After resuspension in fresh media, cells in both conditions were cultured with the $100 \mathrm{ng} / \mathrm{mL} L P S$ challenge for $60 \mathrm{~min}$. Cells were lysed and subsequently analyzed by Western Blot to determine the concentration of $p 65$, the active subunit of $N F_{K} B$, between conditions. Beta actin was used as a loading control. A representative blot is demonstrated showing decreased p65 concentration in the impaired monocyte $(\boldsymbol{A})$. This reached statistical significance, ${ }^{\star} p<0.05(B) . N=6$. A representative phosflow histogram (C) demonstrates a higher peak of NFKB (p65) phosphorylation in naïve cells (green) compared to impaired cells (red) at 30 min after a $100 \mathrm{ng} / \mathrm{mL}$ LPS challenge. 
Figure 22. IkB signaling in the impaired monocyte.

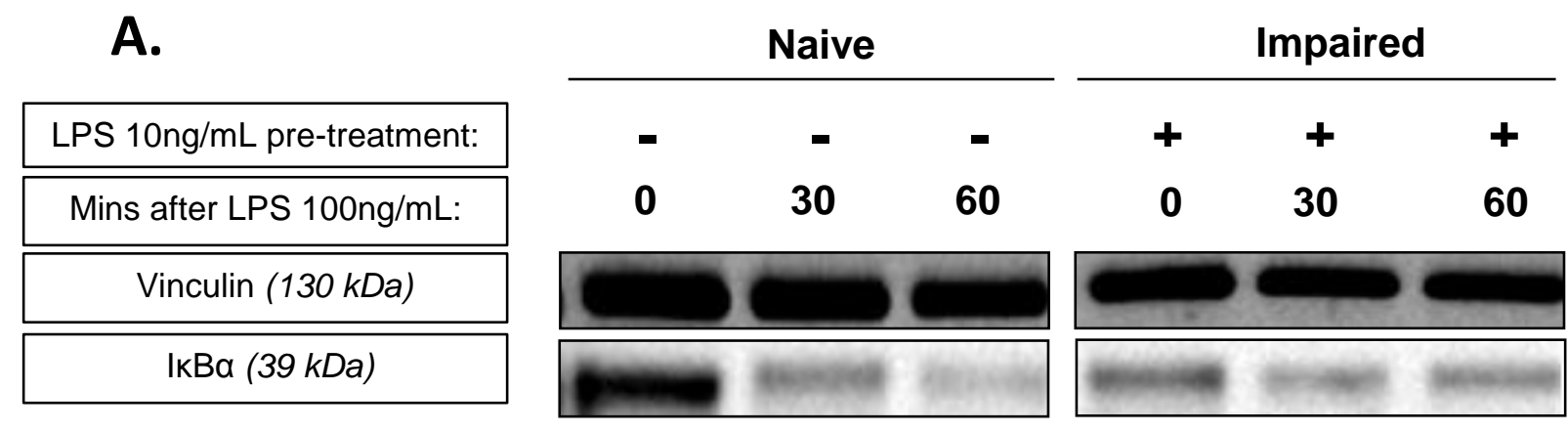

\section{B. Level of IKB}

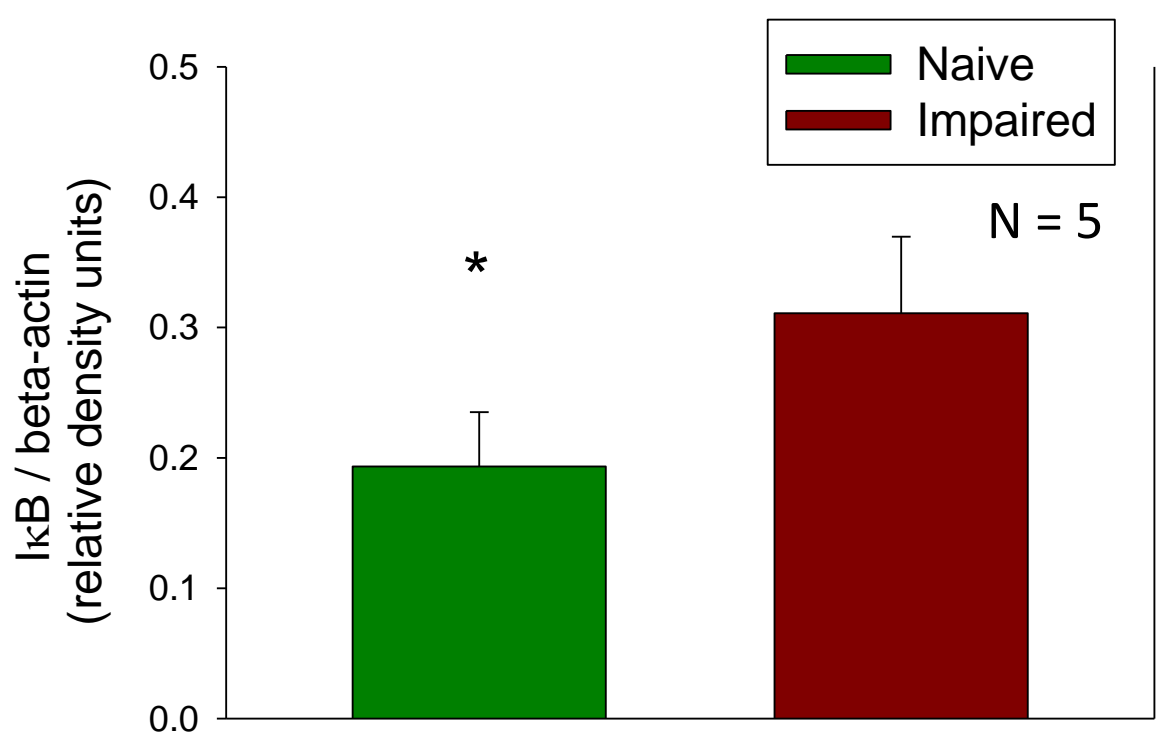

Isolated monocytes were rested for $1 \mathrm{~h}$ and then treated either with media

only or low dose $10 \mathrm{ng} / \mathrm{mL}$ LPS for $16 \mathrm{~h}$. Following washing and resuspending in fresh media, cells in each condition were challenged with $100 \mathrm{ng} / \mathrm{mL}$ LPS and NFKB was measured. Vinculin was used as a loading control. Representative Western Blots $(A)$ demonstrate the effective degradation of IKB in naïve cells (green) by 60 min after the LPS challenge. By comparison, the impaired cells were less effective in IKB degradation by $60 \mathrm{~min}(B$, * p<0.05, Wilcoxon Signed rank Test). $N=5$. 


\section{Total levels of IKK- $\alpha$ \& $-\beta$ in the impaired monocyte}

Messenger RNA levels of the two critical catalytic subunits of IKK, $-\alpha$ and $\beta$, were measured by qRT-PCR at 17 hours to examine for differences in gene expression of IKK at the time of the LPS $100 \mathrm{ng} / \mathrm{mL}$ challenge (Figure 23). Impaired monocytes had decreased IKK- $\alpha$ messenger RNA relative to naïve monocyte. There were no differences between IKK- $\beta$ gene expression between naïve and impaired conditions.

Despite the decrease in IkK- $\alpha$ mRNA, Western blot measurements did not show differences in total protein between naïve and impaired monocytes (Figure 24). In addition, there were no differences in IKK- $\beta$ protein between naïve and impaired cells by Western Blot (Figure 24).

Immunofluorescence was used by qualitatively examining the fluorescence of IKK- $\alpha$ and $-\beta$ using Texas Red between naïve and impaired monocytes. There were no clear differences between naïve and impaired monocytes studied 30 minutes after the $100 \mathrm{ng} / \mathrm{mL}$ LPS challenge.

\section{IKK phosphorylation in the impaired monocyte}

Naïve and impaired monocytes were studied immediately before the 100 $\mathrm{ng} / \mathrm{mL}$ LPS challenge, and then every 15 minutes for 120 minutes to determine differences in IKK phosphorylation between conditions. In Figure 26, IKK phosphorylation in the naïve monocyte occurred maximally at 30 and 45 minutes, which then returned to unstimulated levels by 60 and 120 minutes following the $100 \mathrm{ng} / \mathrm{mL}$ LPS challenge. By comparison, IKK phosphorylation was decreased throughout the 120 minutes of study when compared against the naïve response. 
Figure 23. IkK- $\alpha$ \& - $\beta$ gene expression in the impaired monocyte.

\section{KK mRNA gene expression in the impaired monocyte}

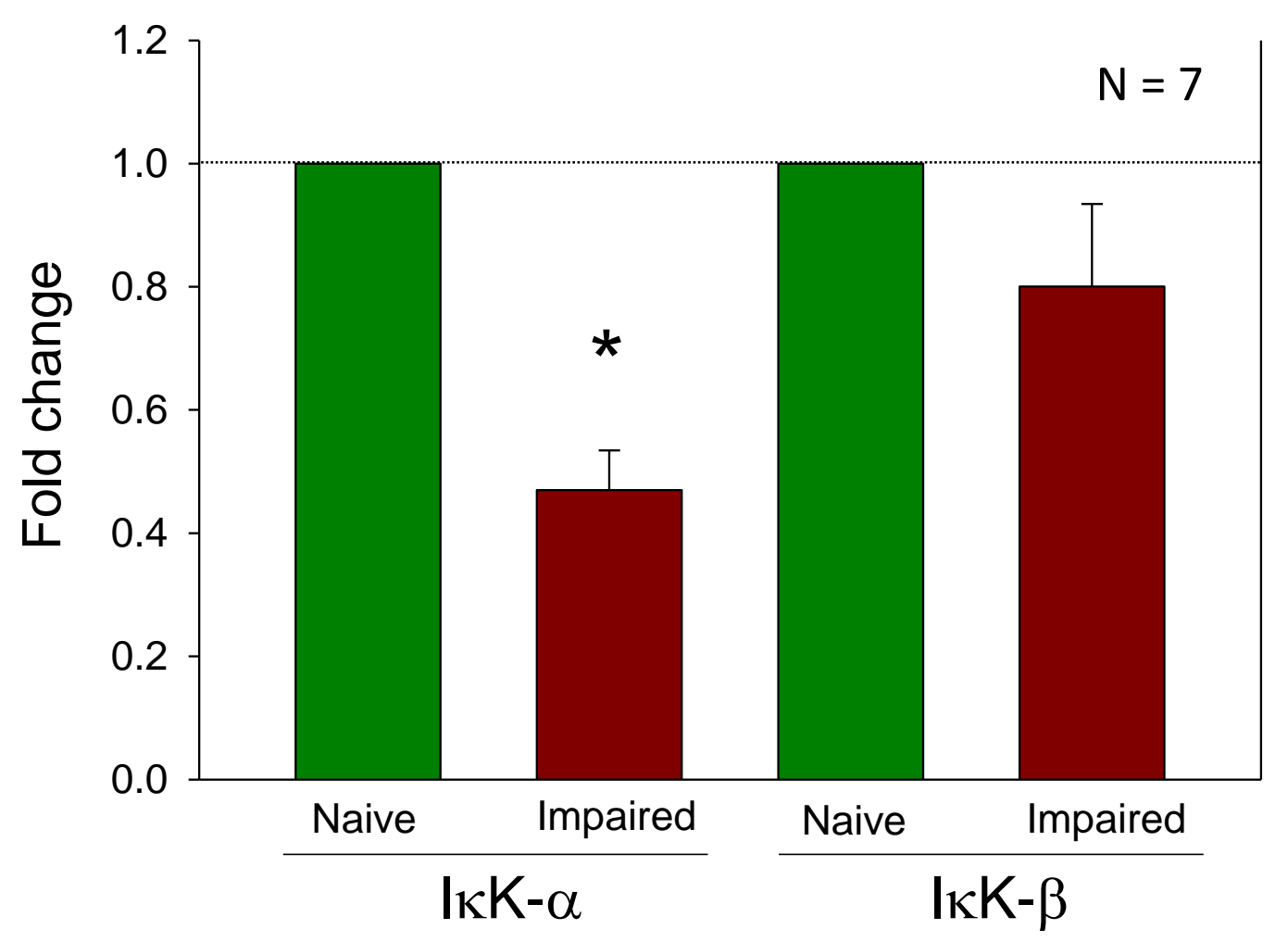

Monocytes cultured in either naïve (media only) or impaired (10 ng/mL LPS) conditions for $16 \mathrm{~h}$ following after an initial $1 \mathrm{~h}$ rest. At $17 \mathrm{~h}$, which would represent the time of $100 \mathrm{ng} / \mathrm{mL} L P S$ challenge, monocyte $R N A$ was analyzed for differences in the expression of IKK- $\alpha$ \& IKK- $\beta$ mRNA using qRT-PCR. Impaired monocyte IKK mRNA levels were expressed relative to naïve cells. These data show that IKK- $\alpha$ is downregulated in the impaired monocyte ( $p$ $<0.05$, Wilcoxon-signed rank test). Gene expression of IKK- $\beta$ did not differ between naïve and impaired conditions. $N=7$. 
Figure 24. IkK- $\alpha$ protein in the impaired monocyte.

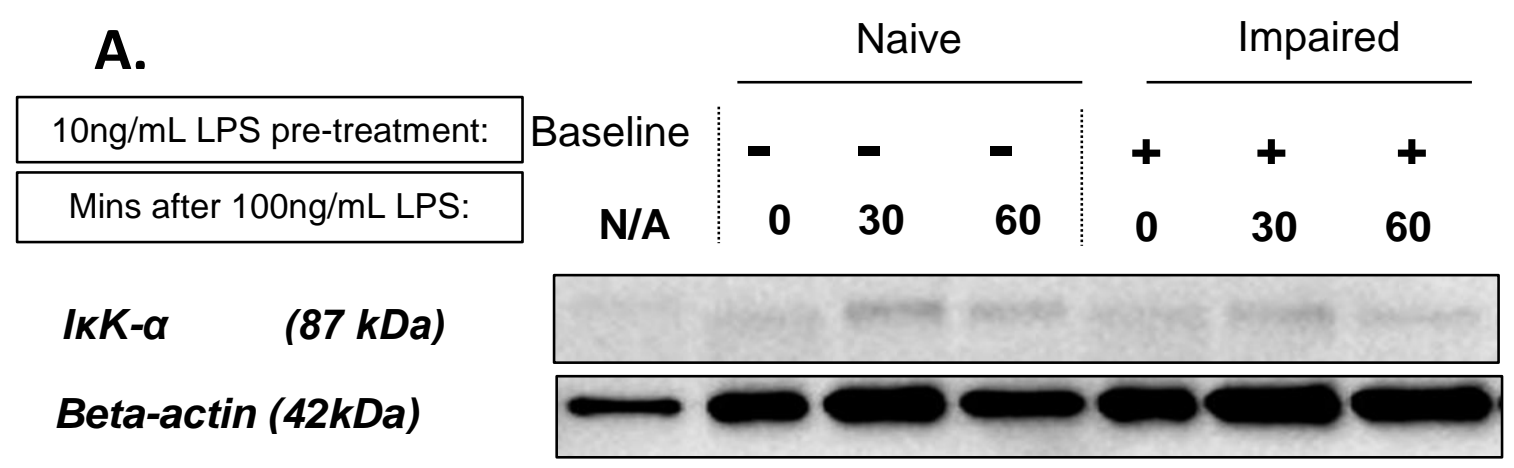

B.

IKK- $\alpha$ protein expression

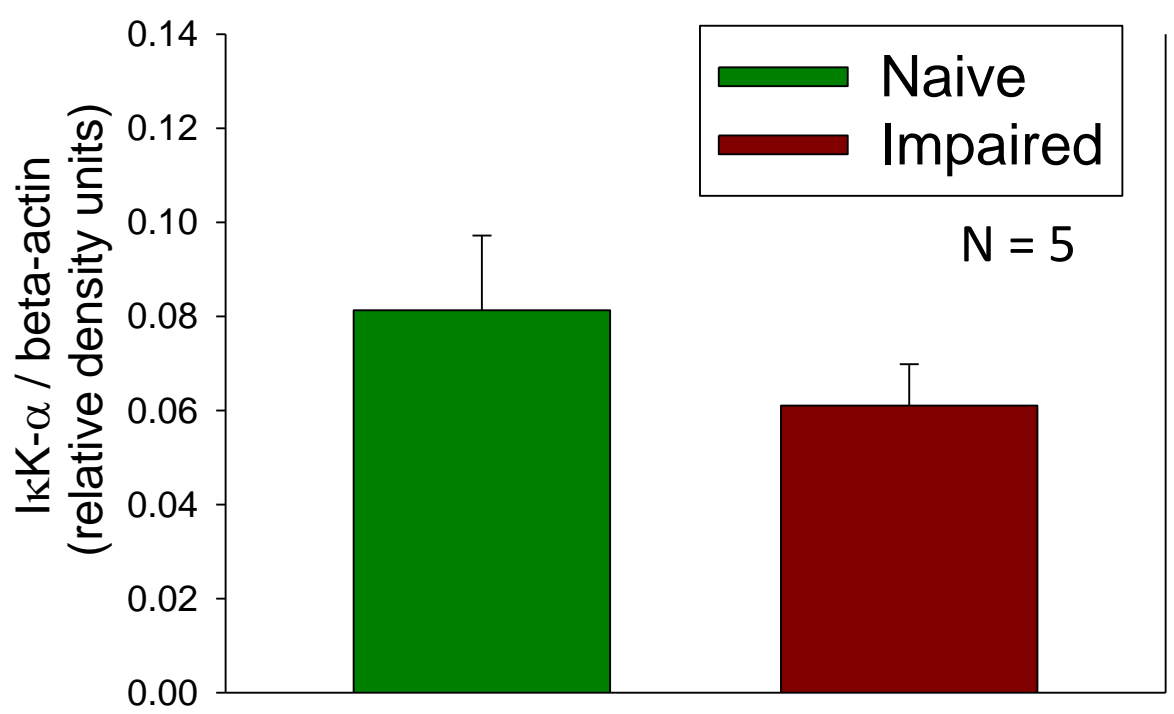

IKK- $\alpha$ protein levels were measured in naïve and impaired monocytes by

Western Blot, using beta-actin as an internal control. Monocytes were cultured in either media only (naïve) or $10 \mathrm{ng} / \mathrm{mL}$ LPS (impaired) for $16 \mathrm{hrs}$. After washing, cells were then treated with the $100 \mathrm{ng} / \mathrm{mL} L P S$ challenge. IKK- $\alpha$ was measured by SDS-PAGE (Western Blot) at the time of isolation, at the time of LPS challenge and 30 and 60 min after the LPS challenge (A). There were no significant differences between naïve and impaired conditions in terms of IKK- $\alpha$ protein concentration at the time of the LPS challenge (17 $h$ ) (B). 
Figure 25. IkK- $\beta$ protein in the impaired monocyte.
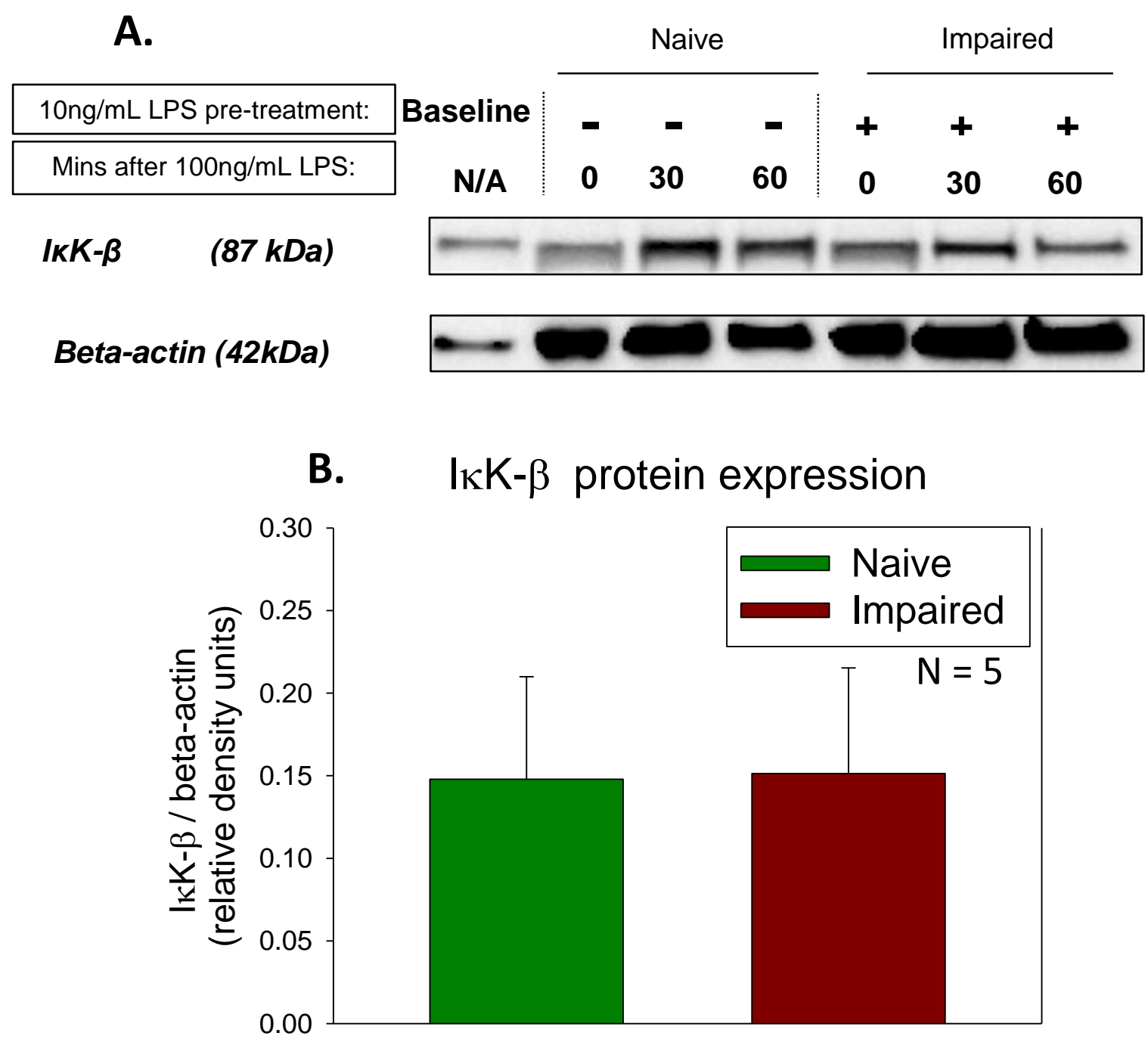

IKK- $\beta$ protein levels were measured in naïve and impaired monocytes by

Western Blot, using beta-actin as an internal control. Monocytes cultured with either media only (naïve) or $10 \mathrm{ng} / \mathrm{mL}$ LPS (impaired) for $16 \mathrm{hrs}$. After washing, cells were then treated with $100 \mathrm{ng} / \mathrm{mL}$ LPS challenge. IKK- $\beta$ was measured by SDS-PAGE (Western Blot) at the time of isolation, at the time of LPS challenge and 30 and 60 min after the challenge (A). No significant differences between naïve and impaired conditions in terms of IKK- $\beta$ protein concentration at the time of the LPS challenge (17 h) (B). 
Figure 26. IkK phosphorylation in the impaired monocyte.

A.

\begin{tabular}{|c|}
\hline $\begin{array}{c}\text { 10ng/mL LPS } \\
\text { pre-treatment: }\end{array}$ \\
\hline $\begin{array}{c}\text { Mins after } 17 \mathrm{~h} 100 \mathrm{ng} / \mathrm{mL} \\
\text { LPS challenge: }\end{array}$ \\
\hline $\begin{array}{c}\text { Phospho-IKK } \alpha / \beta \\
\text { (85-87 } \mathrm{kDa})\end{array}$ \\
\hline $\begin{array}{c}\text { Beta-actin } \\
(42 \mathrm{kDa})\end{array}$ \\
\hline
\end{tabular}

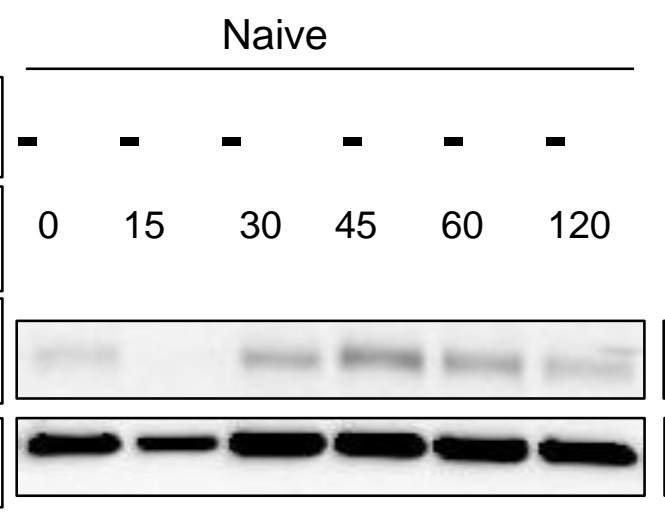

\begin{tabular}{rrrrrrr}
\multicolumn{7}{c}{ Impaired } \\
\hline+ & + & + & + & + & + \\
0 & 15 & 30 & 45 & 60 & 120 \\
\hline & & & & \\
\hline & & & & \\
\hline
\end{tabular}

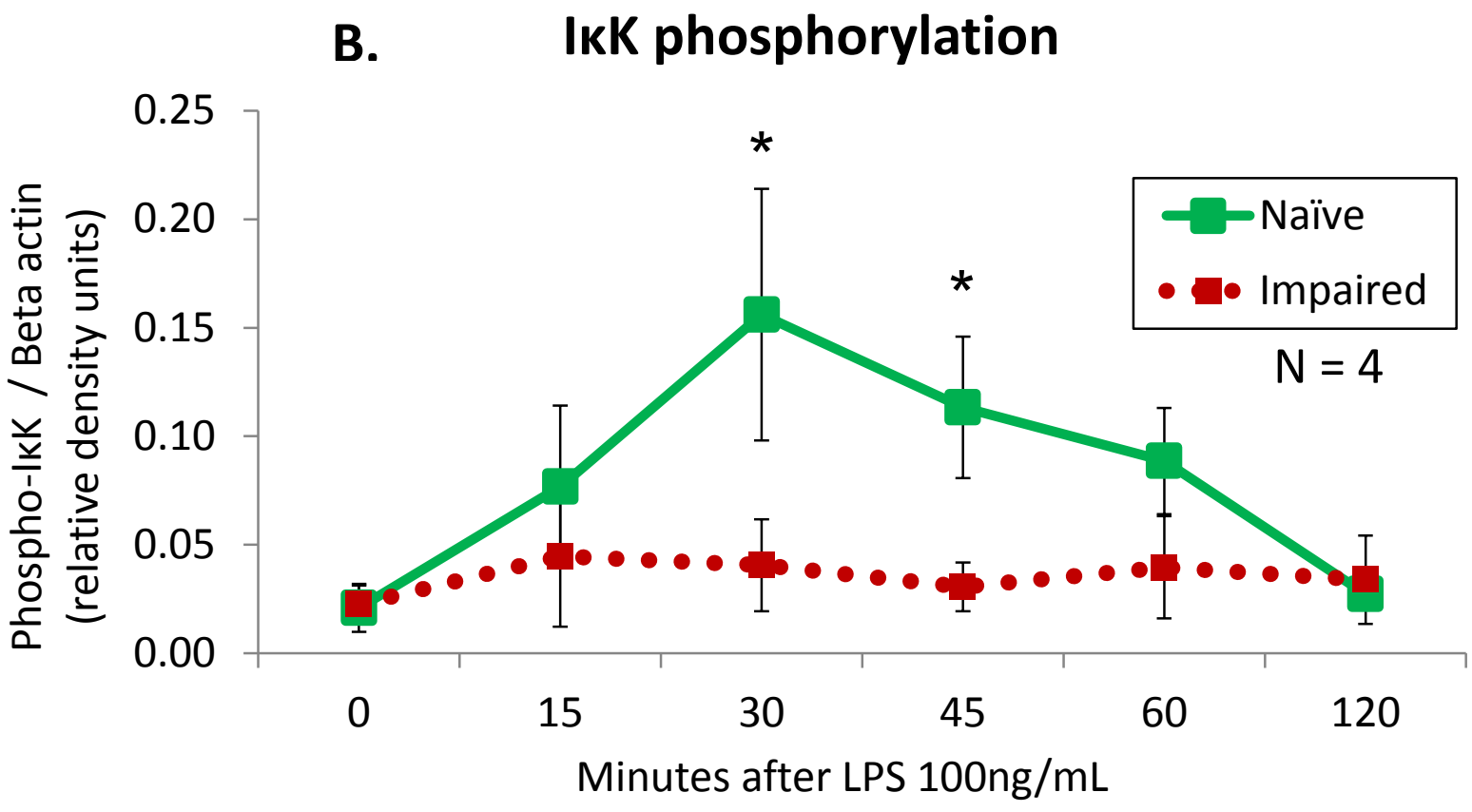

Monocytes were incubated in either naïve (media only) or impaired $(10 \mathrm{ng} / \mathrm{mL}$ LPS) conditions for $16 \mathrm{~h}$ before washed and resuspended. At this point, 100 $\mathrm{ng} / \mathrm{mL} L P S$ was given to both conditions and intracellular monocyte IKK phosphorylation was measured by Western Blot (A). Beta actin was used as a loading control for normalization. IKK phosphorylation was achieved in naïve monocytes maximally at 30 min after the LPS challenge. By comparison, this phosphorylation of $/ K K$ was not seen in cells pre-treated with $10 \mathrm{ng} / \mathrm{mL} L P S$ (impaired). $N=4$. Paired T-test. 
TAK-1 signaling in the impaired monocyte

Both phosphorylated and total levels of TAK-1 were measured over the same 15 minute intervals after the $100 \mathrm{ng} / \mathrm{mL}$ LPS challenge. There were no consistent differences in the phosphorylated TAK-1 levels (expressed as a percentage of total TAK-1) or total TAK-1 levels between naïve and impaired monocytes (Figure 27).

\section{Toll-like receptor signaling in the impaired monocyte}

Naïve and impaired monocytes were profiled for dysregulated TLR signaling 2 hours after the $100 \mathrm{ng} / \mathrm{mL}$ LPS challenge. Two of the 44 genes examined reached statistical significance and were both were downregulated in the impaired monocyte when compared to the naïve cell (Figure 28). These included TRAF-6, equating to approximately 5-fold downregulation, and NFKBIE by 1.6-fold downregulation in the impaired monocyte.

Of interest, the gene for CD14 approached significance at $p=0.075$, with 1.8fold downregulation. IKK- $\mathrm{Y}$ also decreased with a 1.5-fold downregulation $(\mathrm{p}=$ 0.078). 
Figure 27. TAK-1 signaling in the impaired monocyte.
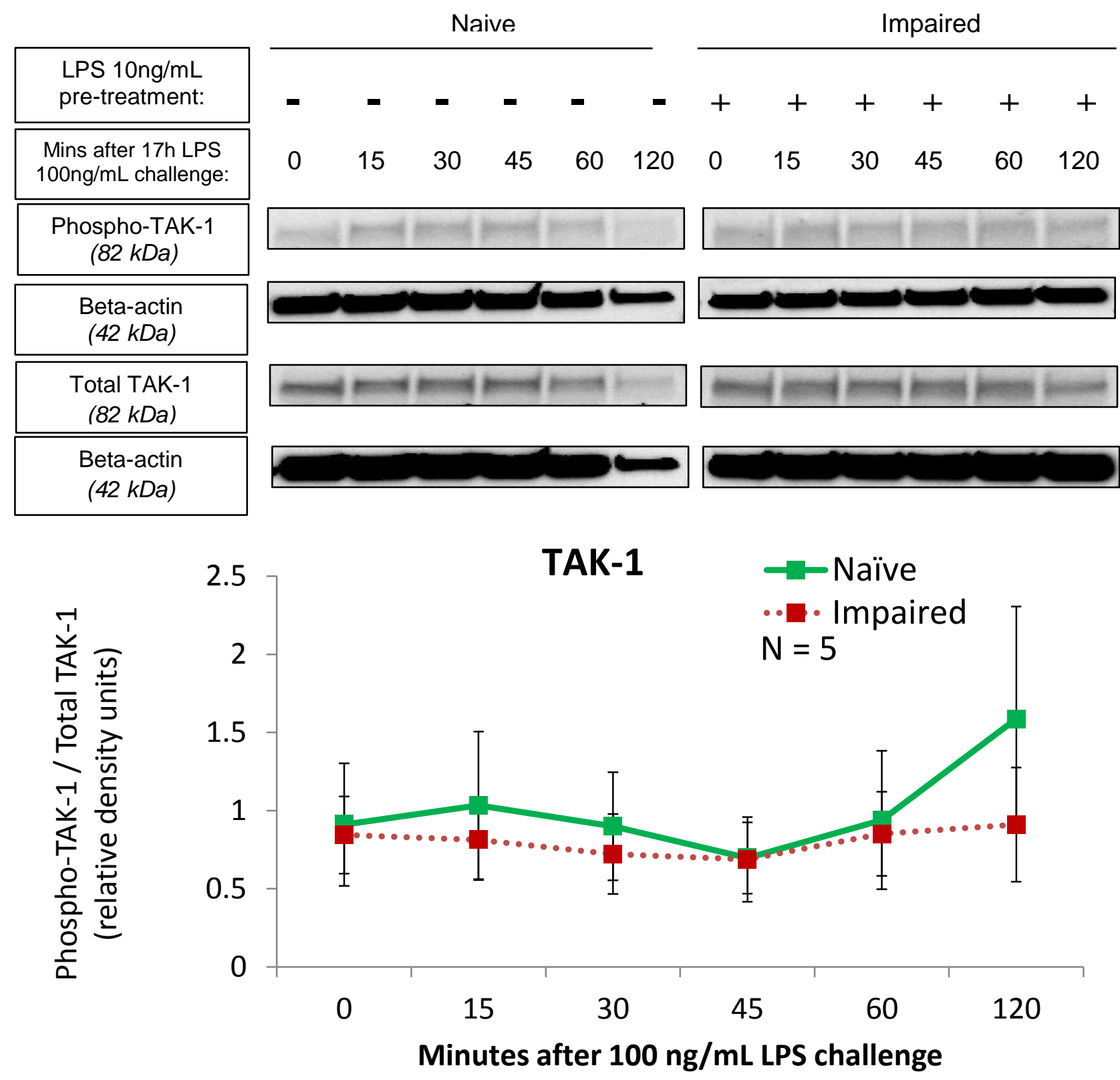

Monocytes were incubated in either naïve (media only) or impaired (LPS

$10 \mathrm{ng} / \mathrm{mL}$ ) conditions for $16 \mathrm{~h}$ before $100 \mathrm{ng} / \mathrm{mL}$ LPS challenge. Intracellular

monocyte TAK-1 (phosphorylation and total) was then measured by Western

Blot every 15 min until 120 min (A). No differences between naïve and

impaired monocytes were elucidated in levels of TAK-1 activation, expressed

as a ratio of Phospho-TAK-1 over Total TAK-1 (after both were normalized

with beta actin). $N=5$. 
Figure 28. Toll-like receptor signaling in the impaired monocyte.

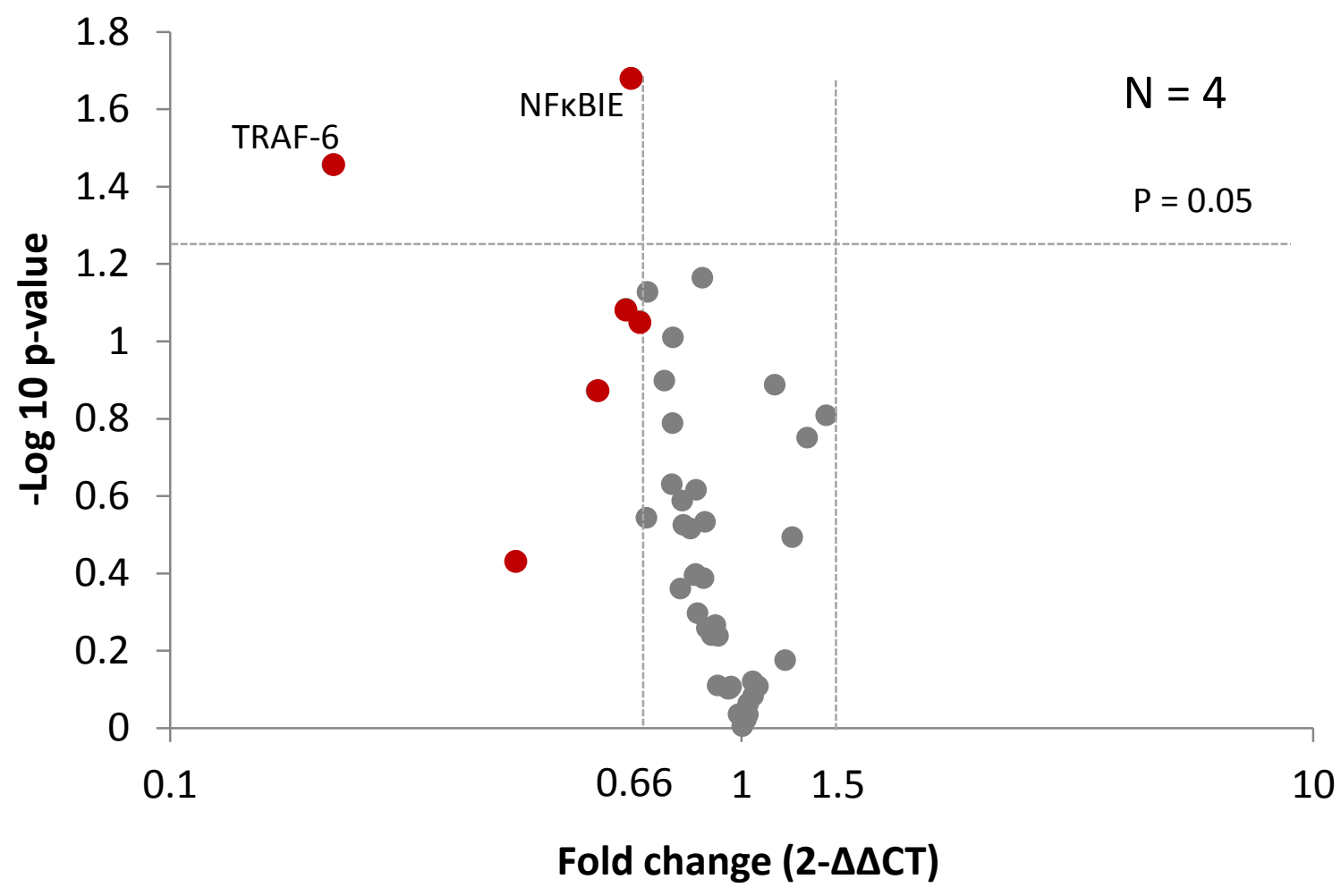

Monocytes were cultured in naïve (media only) or impaired (low dose 10 $n g / m L ~ L P S)$ conditions for $16 \mathrm{~h}$. After resuspension in fresh media, cells in each condition were stimulated for a further 2 hours with the $100 \mathrm{ng} / \mathrm{mL} L P S$ challenge. Intracellular RNA was assessed for gene expression of TLR signaling molecules (mRNA) using TLR profiling assays (qRT-PCR). 18S, GAPDH and beta-2-microglobin were used as internal controls. $N=4$. Each dot represents a gene target. In this volcano plot, genes to the left of the vertical 0.66 line were considered downregulated (equivalent to a fold downregulation of 1.5) and those to the right of vertical 1.5 line were considered upregulated in the impaired monocyte relative to naïve conditions (control). On the $y$-axis, target genes above the horizontal line at 1.3 were considered significant (thus above line equivalent to $p<0.05$, Paired $T$-test). The most consistently downregulated genes were TRAF-6 and NFKBIE. 


\section{IKK-16 as an effective inhibitor of IKK phosphorylation}

Following pre-treatment with either $100 \mathrm{nM}$ IKK-16 (diluted in DMSO) or the equivalent dilute DMSO control for 1 hour after isolation, monocytes were stimulated with $100 \mathrm{ng} / \mathrm{mL}$ LPS. Time points for measurement of IKK phosphorylation by Western Blot were taken at 1 hour (immediately before $100 \mathrm{ng} / \mathrm{mL}$ LPS) and then at 15 minute intervals for 120 minutes. Figure 29 demonstrates that control monocytes (with dilute DMSO only) responded to the $100 \mathrm{ng} / \mathrm{mL}$ LPS challenge with a peak of IKK phosphorylation after 15 minutes. By comparison, levels of IKK phosphorylation were decreased in the IKK-16 treated cells even prior to the addition of $100 \mathrm{ng} / \mathrm{mL}$ LPS, and remained lower at 15 and 30 minutes after the $100 \mathrm{ng} / \mathrm{mL}$ LPS challenge as compared to the control.

Monocyte viability was measured at 17 hours following a range of IKK-16 doses, ranging from a control without LPS, control (DMSO) with LPS, and then 1 up to $1000 \mathrm{nM}$ concentrations of IkK-16. There were no differences in monocyte viability between conditions, with approximately $75 \%$ of cells viable at 17 hours (Figure 30 ).

\section{Effect of IKK-16 on cytokine production}

Under the same conditions, levels of TNF- $\alpha$ were measured by ELISA to determine if targeted IKK inhibition would decrease TNF- $\alpha$ production. Indeed, with increasing doses from 1 to $1000 \mathrm{nM}$, TNF- $\alpha$ was incrementally decreased compared to the DMSO control, reaching statistical significance at the $100 \mathrm{nM}$ and $1000 \mathrm{nM}$ doses (Figure 31). 
The same inhibitory effect of IKK-16 was observed with respect to IL-10 levels. There was more variability in IL-10 production between donors, but IKK-16 treatment decreased $\mathrm{IL}-10$ production at doses of $100 \mathrm{nM}(\mathrm{p}=0.078)$ and $1000 \mathrm{nM}$ doses $(\mathrm{p}<0.05)$ (Figure 32). 
Figure 29. Effect of IKK-16 on IKK phosphorylation.
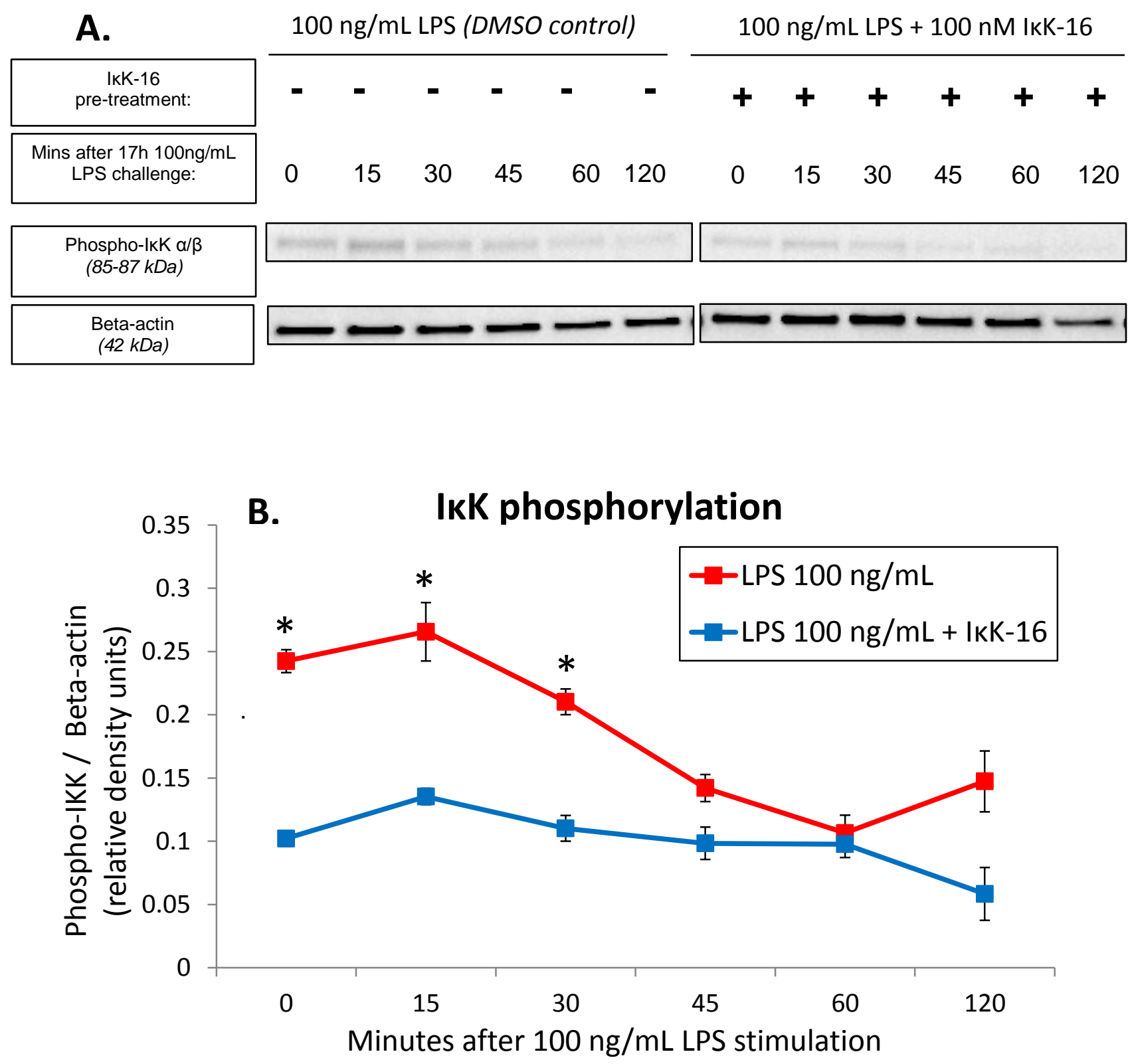

IKK-16 is a selective inhibitor of IKK. Following isolation of monocytes, cells were divided into either $100 \mathrm{nM}$ IKK-16 (which is diluted into a very low concentration of DMSO) or control with DMSO only for $1 \mathrm{~h}$. IKK- $\alpha / \beta$ phosphorylation was determined by Western Blot after the addition of 100 $n g / m L$ LPS (given at 1 h). Beta-actin was used as a loading control. IKK-16 effectively decreases IKK phosphorylation, shown as a representative Blot (A) and quantitatively using four different donors (B). ${ }^{*} p<0.05$, Paired T-test. 
Figure 30. Effect of IKK-16 on monocyte viability.

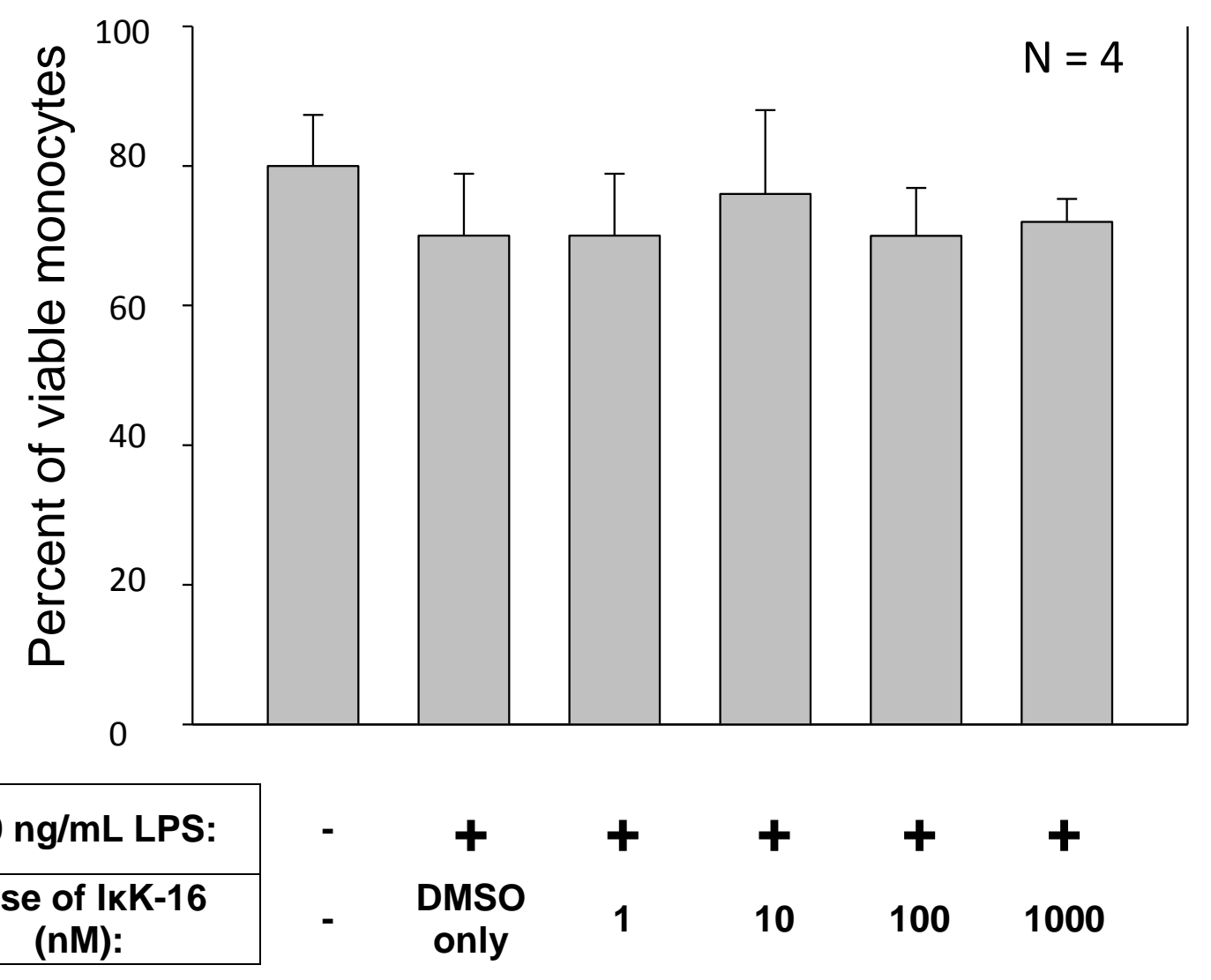

Monocytes cultured with IKK-16 (diluted in DMSO) at various doses for $1 \mathrm{~h}$, then stimulated with $100 \mathrm{ng} / \mathrm{mL}$ LPS for $16 \mathrm{~h}$. At this point, cells were stained with Trypan Blue and manually counted by standard microscopy for number of viable cells, expressed as a percentage of the initial cell number. This experiment demonstrates that neither DMSO nor IKK-16 is toxic to human monocytes. Four donors were used for these experiments. " - "denotes the absence of a reagent, whereas " + denotes the presence of it in cell culture. 
Figure 31. Effect of IKK-16 on TNF- $\alpha$ production.

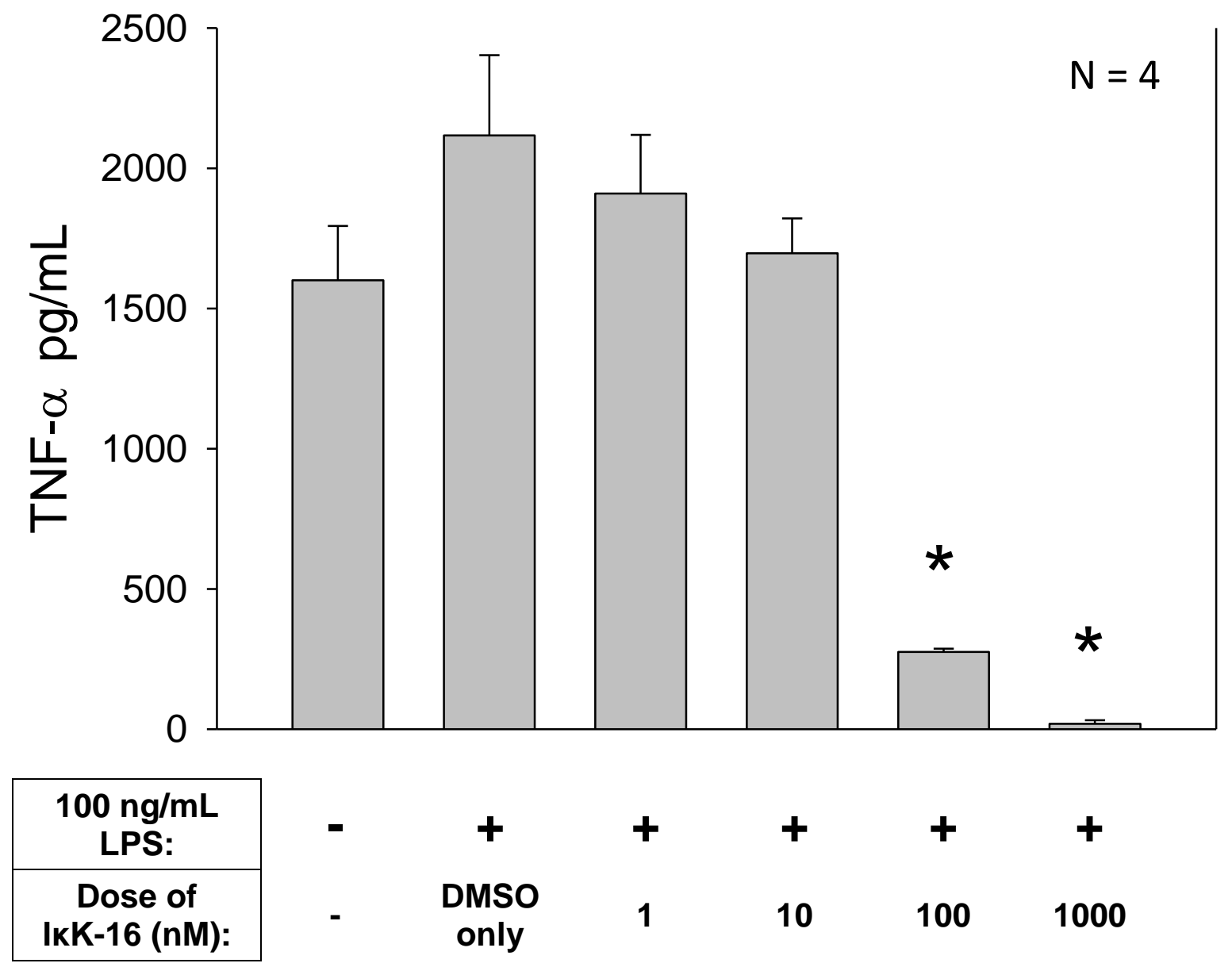

Monocytes were cultured with IKK-16 at various doses (diluted in DMSO) for 1

h, then stimulated with $100 \mathrm{ng} / \mathrm{mL}$ LPS until $17 \mathrm{~h}$. At $17 \mathrm{~h}$, cell culture supernatant was measured for TNF- $\alpha$ protein concentration by ELISA. Both $100 \mathrm{nM}$ and $1000 \mathrm{nM}$ doses of IKK-16 were effective in decreasing TNF- $\alpha$ production ( $p<0.05$, ANOVA with post-hoc Tukey Kramer test). $N=4$. 
Figure 32. Effect of IKK-16 on IL-10 production.

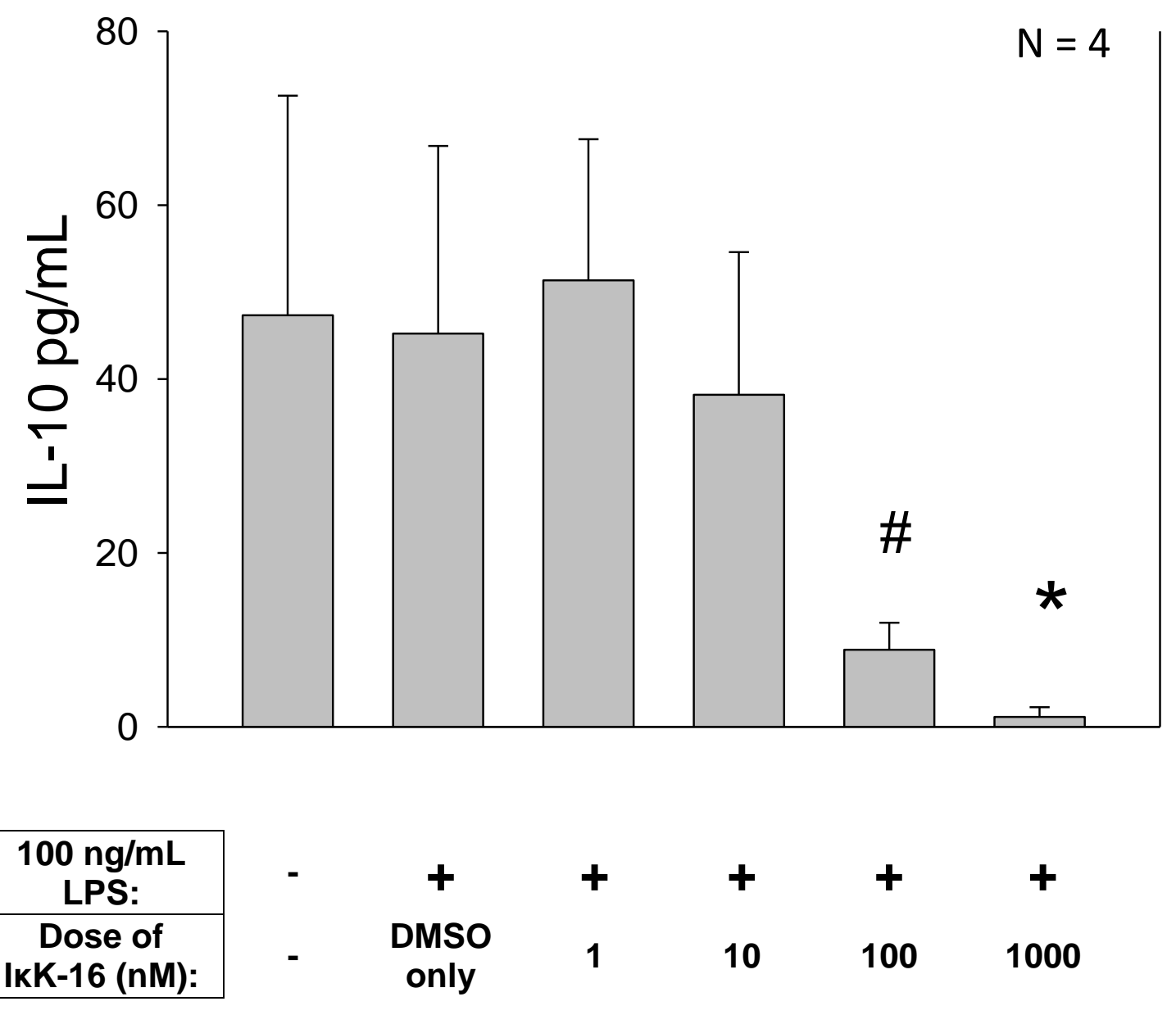

Monocytes were cultured with IKK-16 at various doses (diluted in DMSO) for 1 $h$, then stimulated with $100 \mathrm{ng} / \mathrm{mL}$ LPS until $17 \mathrm{~h}$. At $17 \mathrm{~h}$, cell culture supernatant was measured for IL-10 protein concentration by ELISA. Compared to $1 \mathrm{nM}$, the $1000 \mathrm{nM}$ dose decreased IL-10 levels $\left({ }^{*} p<0.05\right)$. The $100 \mathrm{nM}$ dose also appeared to decrease IL-10 levels compared with $1 \mathrm{nM}$, approaching significance at 0.078 (\#, ANOVA with post-hoc Tukey Kramer test). $N=4$. 


\section{Effect of IKK-16 on HLA-DR expression}

Isolated monocytes were stimulated with or without IKK-16 for 1 hour, before the $100 \mathrm{ng} / \mathrm{mL}$ LPS challenge was added for 16 hours to determine the effect of IKK on HLA-DR expression. We found no differences in the levels of HLADR expression between those treated with or without IkK-16, demonstrated in Figure 33. This is clear from quantitative data from 6 donors, but also shown qualitatively by scatterplots and histograms produced by flow cytometric analyses.

\section{MicroRNA expression in the impaired monocyte}

Screening experiments were undertaken to examine the expression of 384 different microRNAs between the naïve and impaired monocyte. Initial experiments were undertaken at the 33 hour time point (which equates to 16 hours after the $100 \mathrm{ng} / \mathrm{mL}$ LPS challenge). Significantly dyregulated miRNA's included miRNA-487a, -433, -655 and -450a. Those approaching significance with biological significance included miRNA-210, -19b, -29b, -9, -7a, -221 and -16. However, verification with single assay miRNA verification did not demonstrate any of these to be significantly dysregulated (data not shown). Further screening was undertaken at the earlier $17 \mathrm{~h}$ time point (i.e. the time of the $100 \mathrm{ng} / \mathrm{mL}$ LPS challenge). These results revealed that miRNA-155 was the most consistently dysregulated, with miRNA-214 and miRNA-519e also reaching statistical significance (Figure 34). Those approaching statistical significance were therefore included for single assay experiments and included miRNA-10a, -885-5p, -362-3p, -150, -133b, -138, and -212. However, on single assay verification, only miRNA-155 was significantly different between naïve and impaired monocytes at 17 hours (Figure 35). 
Both naïve and impaired monocyte expression of miRNA-155 was upregulated when compared against baseline levels, but low dose $10 \mathrm{ng} / \mathrm{mL}$ LPS resulted in a additive increase to a 12 fold increase compared to baseline, pre-LPS levels.

\section{Effect of IKK-16 on miRNA-155 expression}

We have already shown that miRNA-155 expression increases in response to LPS. When monocytes were treated with $100 \mathrm{nM}$ IKK-16 for 1 hour prior to $100 \mathrm{ng} / \mathrm{mL}$ LPS stimulation, miRNA-155 was suppressed compared to control (Figure 36). 
Figure 33A. Effect of IkK-16 on HLA-DR expression (i).
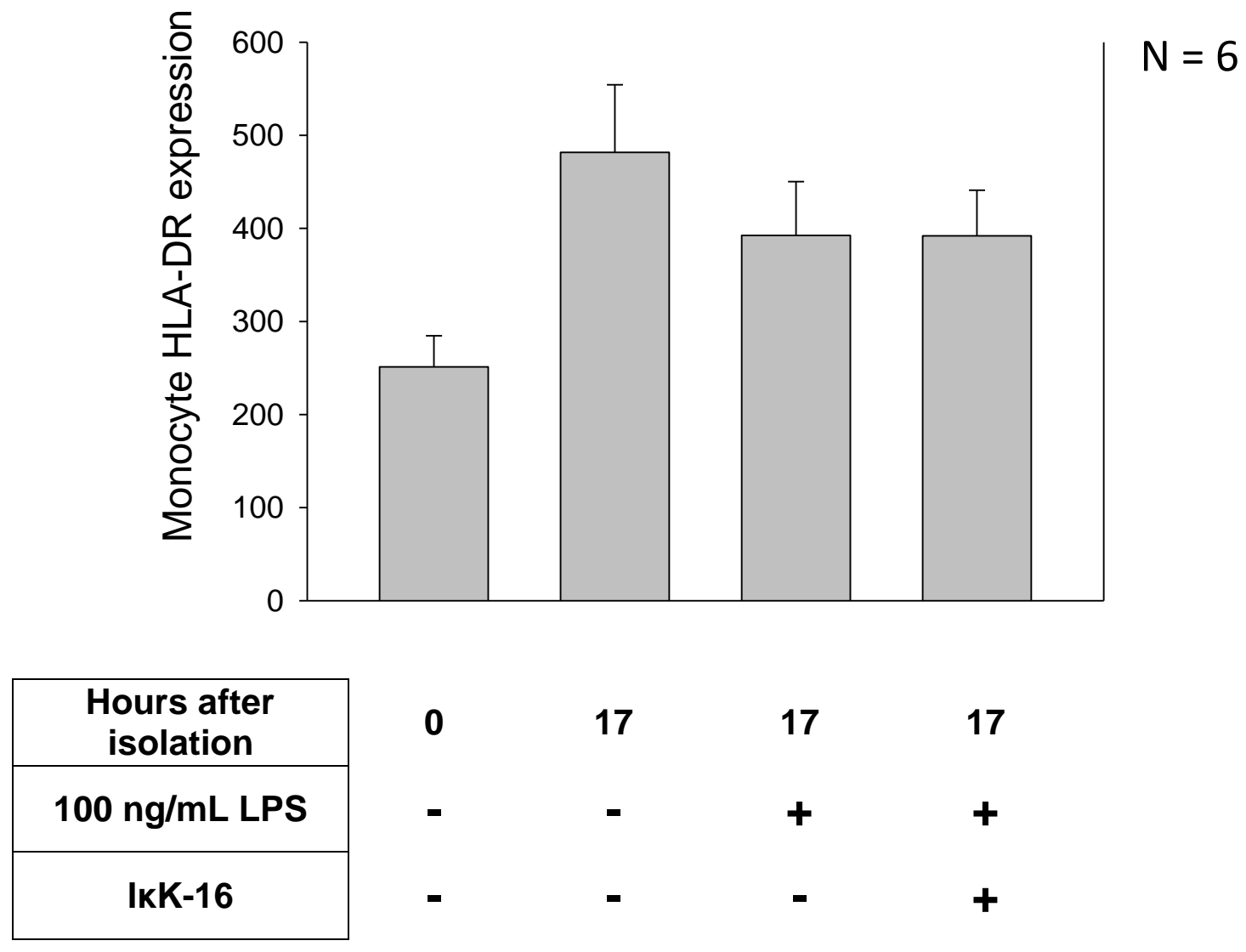

Monocytes were cultured in conditions of either no IKK-16/no LPS, no IKK16/LPS or IKK-16/LPS for $17 \mathrm{~h}$. Cells were analyzed using flow cytometry for HLA-DR expression using a fluorescent PE stain (read on an FL2 channel). Measurements were taken at the time of isolation and at $17 \mathrm{~h}$. In standard cell culture conditions, HLA-DR expression increases by $17 \mathrm{~h}$ from baseline levels. Inhibition of IKK by IKK-16 does not influence HLA-DR expression (i). N =6. This is shown qualitatively (ii) with scatterplots comparing $100 \mathrm{ng} / \mathrm{mL}$ LPS only vs. $100 \mathrm{ng} / \mathrm{mL} L P S+100 \mathrm{nM} / \mathrm{kK}-16$, and via a representative histogram demonstrating no effect if IKK inhibition on HLA-DR (light gray = LPS only, dark gray $=I K K-16 / L P S)$. 
Figure 33B. Effect of IKK-16 on HLA-DR expression (ii).
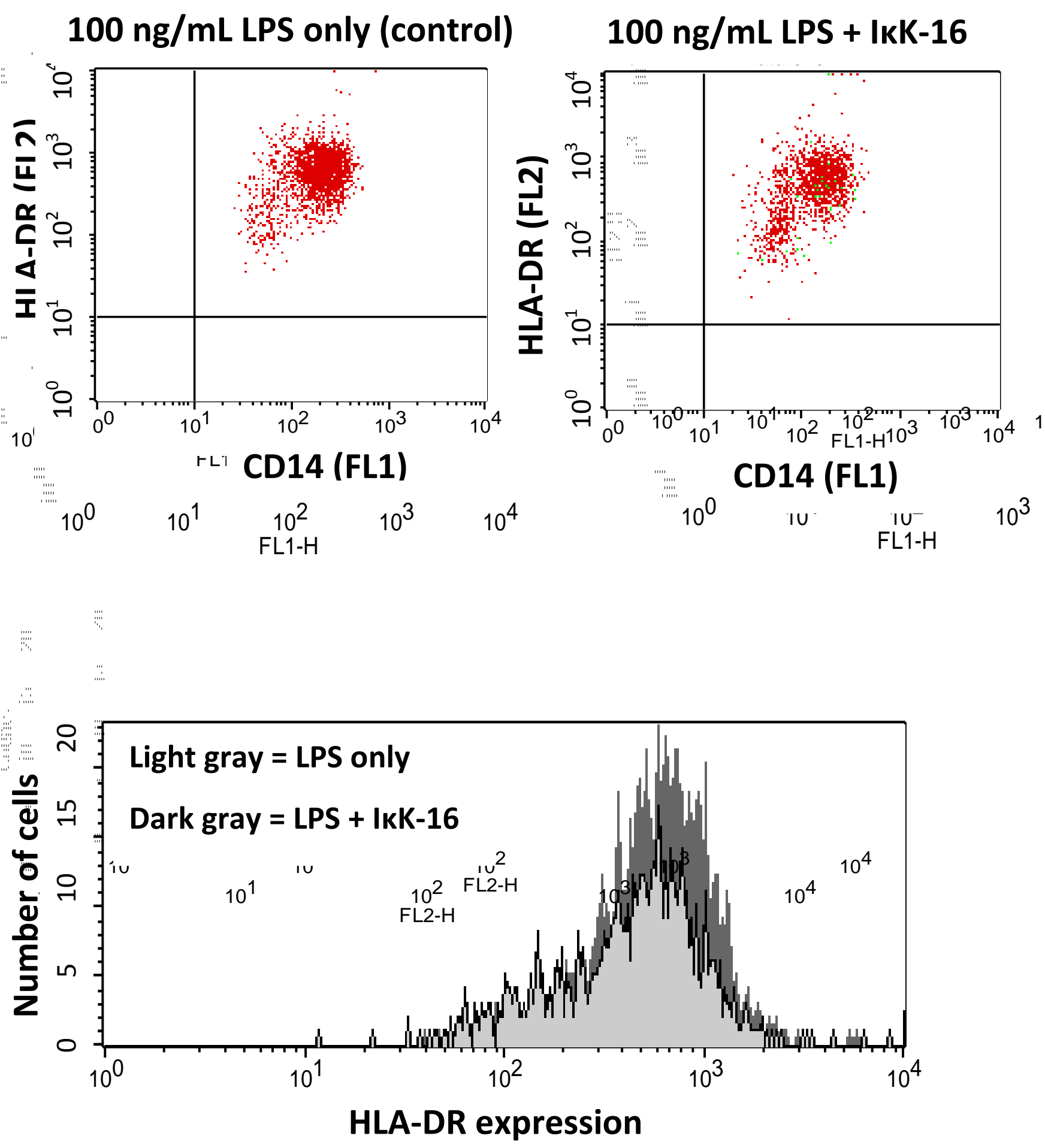
Figure 34. MiRNA screening of the impaired monocyte.

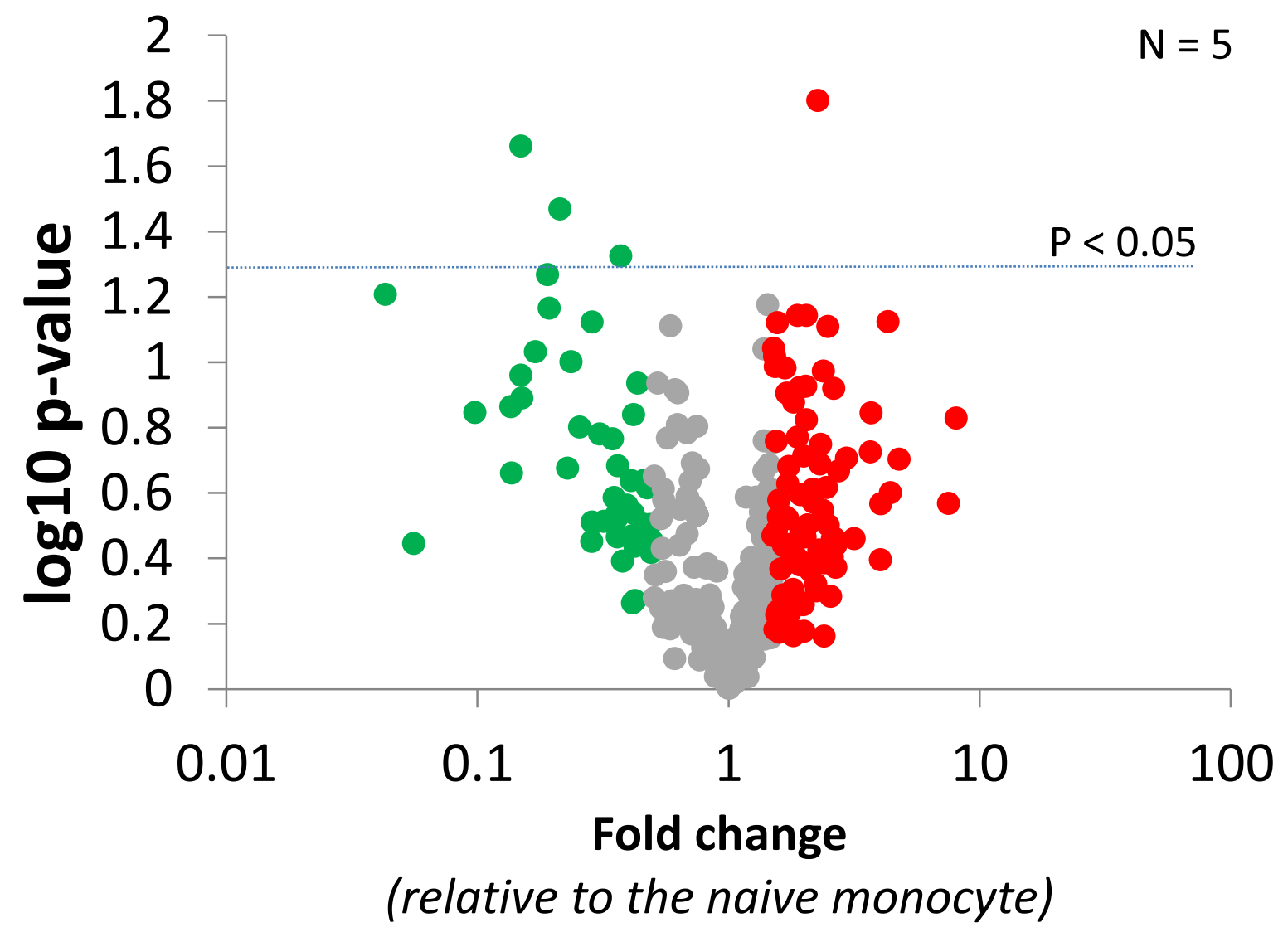

After $1 \mathrm{~h}$ rest, monocytes were cultured for $16 \mathrm{~h}$ in either media only (naïve) or $10 \mathrm{ng} / \mathrm{mL}$ LPS. At $17 \mathrm{~h}$, cells were collected and lysed before RNA was extracted. Following reverse transcription, Taqman Low Density Array cards were used to measure the relative expression of 384 miRNAs by qRT-PCR. A combination of RNU6, RNU44 and RNU48 were used as housekeeping genes for normalization. MiRNA expression in the impaired monocytes were compared to naïve monocytes as their control to express fold changes. Dots to the right (red) represent upregulated miRNAs, whereas dots on the left (green) represent downregulated miRNAs within the impaired monocyte. Paired T-tests were used to generate- $\log _{10} p$-values. A $\log _{10} p$-value of 1.3 is equivalent to 0.05 , thus miRNAs above this level were considered significant. $N=5$. 
Figure 35. Verification of miRNA dysregulation in the impaired monocyte.

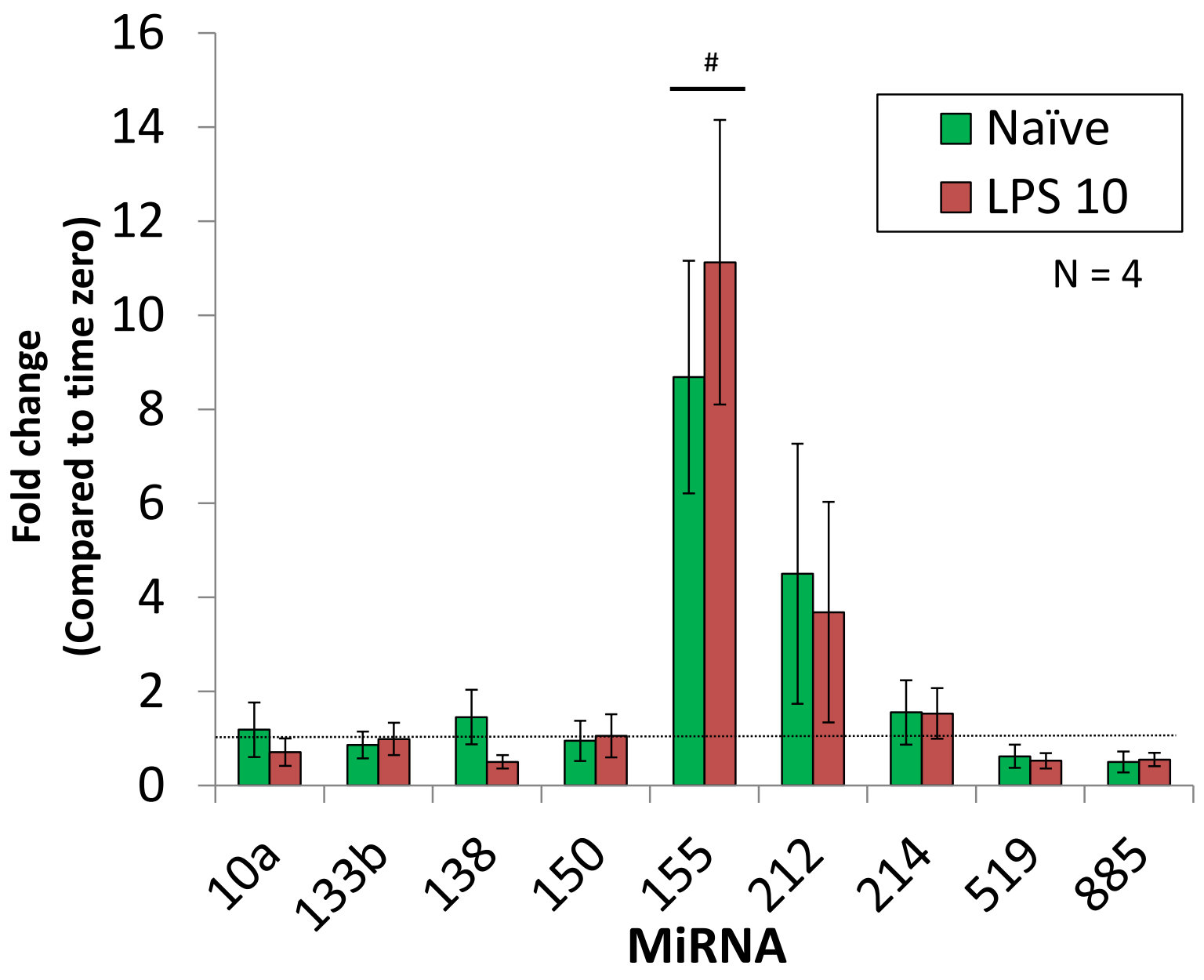

MiRNAs that were significant and approaching significance from the miRNA screening were included for single assay qRT-PCR analysis to verify differences in expression between naïve and impaired monocytes at $17 \mathrm{~h}$. Fold changes here are calculated relative to the baseline level of miRNA expression at isolation. MiRNA-155 is upregulated in both conditions during the $17 \mathrm{~h}$ of cell culture, but low dose $10 \mathrm{ng} / \mathrm{mL}$ LPS results in even greater increase (\# $p=0.08$ ). Other miRNAs assessed were not significantly different between conditions. 
Figure 36. Effect of IkK-16 on miRNA-155 expression.

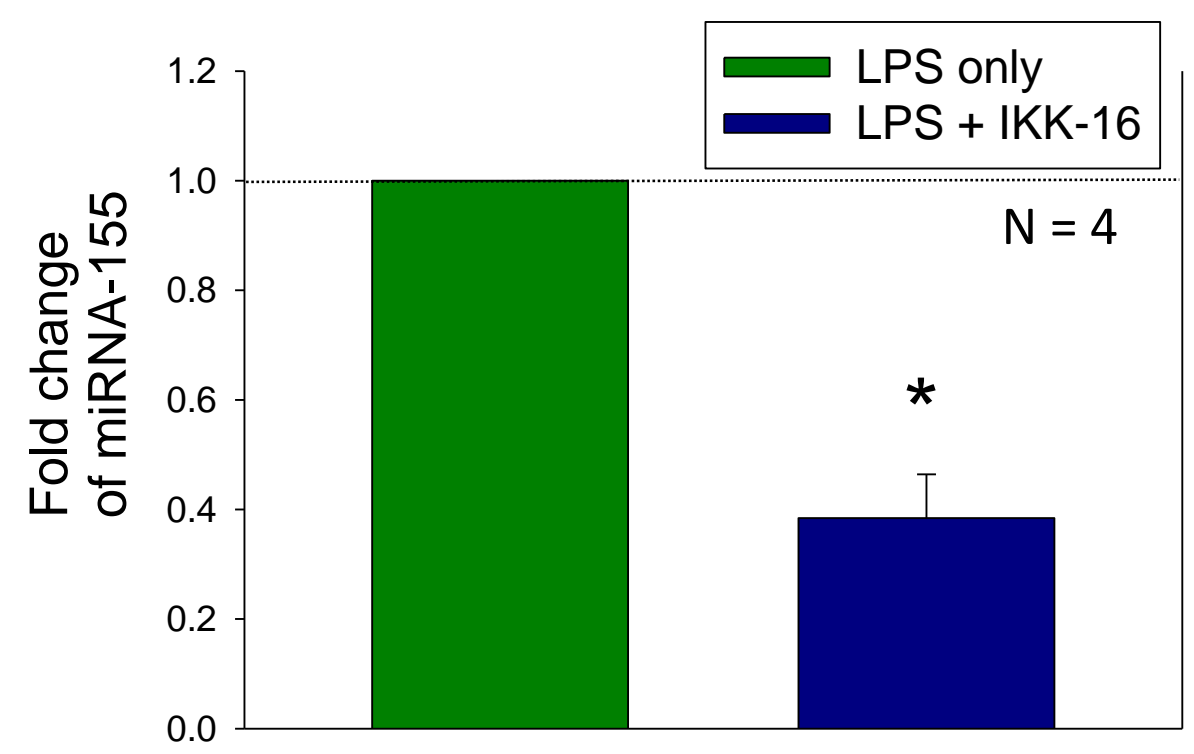

Monocytes in culture following $1 \mathrm{~h}$ of rest were either stimulated with 100 $\mathrm{ng} / \mathrm{mL}$ LPS only (green) or $100 \mathrm{ng} / \mathrm{mL}$ LPS in combination with $100 \mathrm{nM} / \mathrm{kK}-16$ for $17 \mathrm{~h}$. At 17h, monocyte intracellular RNA was extracted and miRNA-155 expression was determined by qRT-PCR. MiRNA-155 levels are expressed relative to control (100 $\mathrm{ng} / \mathrm{mL} L P S+$ dilute DMSO only). This shows that IKK16 blocks miRNA-155 production in response to LPS $\left(^{*} p<0.05\right.$, Paired $T$ test). $N=4$. 


\section{DISCUSSION}

This chapter aimed to understand the mechanisms underpinning how an initial stimulus leads to a subsequent suppressed inflammatory response. The hypothesis was that alteration of NFKB and IKK signaling account for how impaired monocytes have suppressed immune responses. The first set of experiments in this chapter demonstrate decreased levels of total p65, the active subunit of NFKB, decreased p65 phosphorylation and critically, impaired IKB degradation. The three sets of data examine different measures of NFKB functionality and all demonstrate alterations in this important proinflammatory pathway. NFKB is known to be stimulated by LPS and other TLR ligands in order to generate an inflammatory response. It has been established that LPS activates NFKB to produce TNF- $\alpha$, which occurs within the first 17 hours. In response subsequent LPS challenge, the data presented here show that cells with prior LPS exposure have suppressed NFKB activation, decreased gene expression of TNF- $\alpha$ and decreased TNF- $\alpha$ production. This suggests a negative feedback loop presumably evolved to protect excessive, ongoing and potentially harmful responses. Recall the early experiments by Beeson in the 1950's, which showed that repeated endotoxin injections to dogs produced less and less of a fever after each exposure. NFKB suppression would also account for the decreased IL-6 and IL-10 levels also seen in the previous chapter, as these genes are known to be transcribed via this signaling pathway. It should be noted that other pathways exist in pro-inflammatory responses. We have not examined MAPK or ERK/JNK pathways which may also be suppressed. 
The most compelling experiments demonstrate decreased IKB degradation in the impaired cells. This molecule represents "the brakes" within this pathway, which in essence "are left on". In a normal response, the brakes are released by the upstream kinase, IKK. Therefore, these results imply that there may be suppressed IKK function which might account for this defect.

Our experiments began focusing on the gene expression of the catalytic subunits, IKK- $\alpha$ and $-\beta$. Recall that in the Glue Grant studies discussed earlier, IKK- $\beta$ was downregulated in trauma patients compared to healthy controls. Our results do not show differences in IKK- $\beta$ gene expression or indeed protein levels in the impaired monocyte. Conversely, IKK- $\alpha$ was consistently downregulated in the impaired monocyte in terms of gene expression. However, this difference was not proven in the protein levels at 17 hours. Time course Western Blot experiments did not convincingly show differences in IKK- $\alpha$ at later time points. It is not uncommon that differences in messenger RNA are not always shown with the protein product. One possible explanation for this may be the persistence or lack of degradation of the existing protein product within the cells (in essence, changes in the production but not changes in the degradation).

Given the lack of evidence for decreases in the total concentrations of the catalytic subunits of IKK, the next experiments examined the level of activation, i.e. level of phosphorylation of IKK, in response to the $100 \mathrm{ng} / \mathrm{mL}$ LPS challenge. Western Blots every 15 minutes after the challenge revealed clear suppression of IKK phosphorylation in cells with prior LPS exposure, when compared to naïve cells that demonstrated dynamic IKK phosphorylation peaking at 30 and 45 minutes after the LPS challenge. 
Indeed, these experiments address the hypothesis that IKK is suppressed in the impaired cells. However, they demonstrate correlation, but do not in themselves prove causation, which we will address later.

Given that the "chain of activation" via a cascade of phosphorylation has been interrupted and prevented signals downstream, we explored upstream mechanisms. TAK-1 is often described as an intermediate signaling protein between TLR pathways and IKK. However, our experiments do not show any differences in the phosphorylation or total levels of TAK-1 between naïve and impaired monocytes. One should note that TAK-1 is known to be affected by ubiquitination in very complex biochemical protein modifications, which we did not examine as this was beyond the scope of the hypothesis. Furthermore, the input signals from upstream pathways to IkK are redundant and involves more than 15 modifiers including a multitude of kinases and phosphatases. Therefore, we adopted an approach of examining a large amount of TLR related genes using gene expression profiling experiments comparing the impaired monocyte to the naïve. We found that TRAF-6, a gateway to numerous downstream pathways including NFkB, was decreased. TRAF- 6 has previously shown to be decreased in endotoxin tolerance type studies, and more recently has been attributed to posttranslational modification by TNFAIP3 (A20) and miRNA-146a, and is thought to be induced in response to the initial LPS stimulus. Even more interesting is the findings of decreased TRAF- 6 in the impaired monocyte in the TLR profiling experiments. This may in fact reveal a signaling defect between TRAF- 6 and IKK independent of TAK-1. 
Other interesting trends included the downregulation of NFKBIA and CD14. The former represents a gene coding for a subunit of the IkB complex. This is in contrast to expected findings, as in albeit very different models studying TNF signaling, IKB was in fact upregulated in response to external TNF stimulation in order to limit later NFkB activation. However, a trend towards decreased CD14 gene expression would correspond with decreased responses to subsequent LPS stimulation, as CD14 is a co-receptor with MD2 and allows for TLR4 dimerization and signal transduction to MyD88. This represents potentially multiple levels of negative feedback regulation, which demonstrates the redundant nature of the innate immune system.

It should also be noted that there are various other explanations for the decreased responsiveness to LPS. Rather than intra-cellular molecular feedback mechanisms, an internalization of surface membranes and a change in plasma membrane fluidity could result in the lack of available external receptors for LPS, resulting in an impaired response. In addition, this impairment could also be explained by a failure to recruit TLR4 receptors form the cytosol, or other structural "machinery" which is exhausted. This was certainly beyond the scope of this dissertation, but is an interesting area for future research.

Our attention turned back to the role of IKK in monocyte function. There is a correlation between decreased IKK phosphorylation, decreased proinflammatory cytokines and decreased HLA-DR expression in the impaired monocyte. If monocyte impairment occurs as a result of IKK signaling, we wanted to see if IKK inhibition would "turn a naïve monocyte into an impaired monocyte". For these experiments, IKK-16 was used to inhibit IKK 
phosphorylation. Other studies have demonstrated decreased IkK phosphorylation in response to IKK-16. We confirm these findings in Figure 29, which demonstrates a true loss of function with use of this small molecule selective inhibitor. This is of interest not only to demonstrate the mechanistic role of IKK, but IKK-16 has been investigated as a potential therapy for excessive inflammation in animal models of sepsis and hemorrhagic shock. Importantly, any differences seen are due to pathway inhibition, rather than toxicity to cells (Figure 30). Indeed, inhibition of IKK decreases both TNF- $\alpha$ and IL-10 production, as seen in the impaired monocyte, in a dose-dependent fashion. This proves a cause and effect relationship between the suppression of IKK and pro-inflammatory cytokine production, as per the thesis hypothesis. $100 \mathrm{nM}$ IKK-16 appears to be the minimal dose for therapeutic effect.

The same was not true for IKK inhibition and HLA-DR expression. Despite exposure to the same dose of IKK-16 (100 nM) shown to effectively decrease activation of IKK, there was no change in HLA-DR expression compared to control. The expression of HLA-DR has been mostly attributed to transcription of CIITA following JAK-STAT1 signaling, which is known to be activated by IFN- $\gamma$. However, cross-talk between STAT and NFKB pathways does exist, with LPS induced HLA-DR activation previously shown to also depend on NFKB activation ${ }^{116}$. Taken together, it is therefore possible that there are defects of JAK-STAT signaling in impaired monocytes that contribute to decreased HLA-DR expression, rather than a direct result of decreased IKK and NFKB signaling. This has very interesting clinical implications. The use of "blockers" of the inflammatory response of course has concern, and this concern extends to the inhibition of IkK through IKK-16. 
However, these experiments suggest that IKK-16 has the potential to be used as a "dimmer switch" to tune down inflammatory cytokine production but still preserve antigen-presenting functions, as measured by HLA-DR expression. These findings in combination with those by Li et al who found that IKK-16 did not impair phagocytosis suggest that IKK-16 would decrease cytokine responses by monocytes but not the other functions such as antigen presentation and phagocytosis.

MiRNAs are small, non-coding single stranded RNA sequences in their mature form which cause post-transcriptional inhibition of protein production. There has been a great deal of research into the role of miRNA in a variety of diseases including cancer and inflammation-related conditions. MiRNAs are known to act as powerful regulators of toll-like receptor signaling, and other groups have shown that miRNA-146a is increased in conditions of endotoxin tolerance. In our findings, there was a slight increase in miRNA-146a in the impaired monocytes, but this was not consistent and the magnitude of the change was very small, which therefore did not qualify for single assay verification. By comparison, miRNA-155 was upregulated in both naïve and impaired conditions, but more so in the impaired condition. It has been shown previously that miRNA-155 is increased in response to TLR ligands such as LPS ${ }^{117}$, but has not been associated with impaired monocyte function in the same way as miRNA-146a. More commonly in the literature, miRNA-155 is known for its early pro-inflammatory actions through suppressing its targets of SHP1 and SOCS1 to promote TNF- $\alpha$ and other pro-inflammatory cytokines. However, the delayed effects of miRNA-155 in decreasing the proinflammatory response have also previously been shown. Inflammation- 
related targets also include RelA (p65), MyD88, TAB2 (part of the TAK-1 complex) and IKK- $\varepsilon$ and would explain the role of miRNA-155 in dampening inflammation. Of those targets measured in our experiments, p65 was decreased as assessed by Western Blot at 60 minutes after the LPS challenge, and IKK- $\varepsilon$ trended downward in TLR profiling of the impaired monocyte, in keeping with these miRNA-155 results. Given the higher expression of miRNA-155 in the impaired monocyte and the decrease in miRNA-155 targets, this could in part explain the suppression of IKK and monocyte function in general. Nevertheless, miRNA-155 is associated with a large number of inflammatory diseases and previous research in our lab demonstrated the important role in cytokine regulation ${ }^{54}$. MiRNA-based therapy may be possible in the future, however, in the meantime understanding how current therapeutics may influence miRNA expression as a mechanism of action improves our understanding of positive and negative effects of therapy. Our results demonstrate that IKK inhibition with IKK-16 decreases miRNA-155 expression. This demonstrates mechanistically that miRNA-155 induction is IKK dependent, and that a likely feedback loop exists in our model involving LPS stimulation of IKK and subsequent miRNA-155 induction in the first 17 hours, which in turn inhibits TLR, IKK and NFKB activation in response to the LPS challenge through miRNA-155 mediated inhibition of p65 and the other discussed targets. These results also imply that IKK-16 therapy could limit excessive early inflammatory responses (via decreasing the inhibition from SOCS1 and SHP1), and limit delayed immunosuppression by preventing p65, MyD88 and TAB1 suppression. 
The miRNA screening experiments were designed to "throw the net wide" in an unbiased fashion to find potentially novel miRNAs in the context of monocyte impairment. However, this approach has some limitations. We measured time zero (after isolation), at 17 hours and at 33 hours. It is possible that we may have missed some miRNAs which were transiently changed between these time points. Furthermore, the screening approach carries the risk of both false positive and false negative results. Ideally, if there were a higher number of donors used for screening with larger magnitudes of miRNA differences, a false-discovery rate (FDR) correction such as the BenjaminiHochberg procedure or the method of Jung may have been appropriate to limit the risk of type I error ${ }^{118,119}$. Future studies with gain and loss of function of miRNA-155 would be interesting, however this was beyond the scope of this project and given the relatively small differences of miRNA-155 expression between conditions we turned our attentions downstream in the pathway, as discussed in the next chapter.

In summary, this chapter has focussed on IKK signaling and demonstrated that 1) Impaired monocytes have decreased NFKB activation, 2) Impaired monocytes have decreased IkK phosphorylation 3) naïve cells can become "impaired" by IKK inhibition in terms of cytokine production 4) IKK inhibition does not impair HLA-DR expression. These results demonstrate cause and effect of IKK function and the capacity of the monocyte to produce cytokines in response to an infective challenge. 


\section{CHAPTER VII \\ THE ROLE OF IKK IN THE INFLAMMATORY RESPONSE INTRODUCTION}

Our data thus far demonstrates the critical role of IKK activation and the monocyte inflammatory response. Our results have focused on TNF- $\alpha$, IL-10 and HLA-DR as markers of monocyte function. However, as increasingly complex assays are applied to studying sick surgical patients, a long list of cytokines and markers have been associated with poor outcomes in the surgical patient following trauma.

Certain patterns of cytokine and chemokine levels in patients following major trauma have been shown to predict patient trajectories ${ }^{120}$. In a study by the Pittsburgh group, 44 patients who developed nosocomial infection after trauma were compared to 44 patients who had an uncomplicated recovery. Patient cohorts were matched by age, gender, injury severity score and interventions. Both groups had increases in MCP-1 (CCL2), IL-6, IL-1RA and HMGB1 compared to healthy volunteers, but levels were higher in the group who became infected. Those who suffered later infection also had early and sustained increase in IL-7, IL-5, IL-17A, IL-4, IL-13, MIP-1 $\alpha$ (CCL3), MIP-1 $\beta$ (CCL4), IFN- $y$, IL-15, sIL-2Ra, GM-CSF and IP-10. In sequential observations of both sets of trauma patients, levels of IFN- $\alpha$, IL-1 $1 \beta$, IL-2 and TNF- $\alpha$ were low immediately after injury, but in the group who developed nosocomial 
infection, these levels gradually rose by 24 hours, with persistent elevation up to 7 days after injury. It should be noted that in this study, the mean time to developing infection was 7 days following injury (i.e. after the observed cytokine changes), which suggests that the infection may be a consequence of immune dysregulation, rather than the reverse.

The aims of this experiment were 1) to determine whether specific cytokines were inhibited in the context of monocyte impairment, or whether a global reduction in the capacity to produce cytokines occurs and 2) to determine which cytokines and chemokines were modulated through IKK inhibition, to offer a better understanding of this potential therapeutic target.

\section{METHODS}

\section{Sample size considerations}

Power calculations were undertaken based on prior pilot experiments with data on TNF- $\alpha$, IL-10 and IL- 6 as representative cytokines. The effect size was estimated based upon the mean differences and variability of that difference between the naïve and impaired monocyte at 12 hours following the $100 \mathrm{ng} / \mathrm{mL}$ LPS challenge (Table 5A). This was then used to estimate the required sample size, depending on the minimal detectable difference for the three cytokines (Table 5B). A representative power-sample size curve for IL10 is shown (Figure 37). Based on these calculations, 10 different donors were used for these experiments in order to achieve a power of $0.80(80 \%)$. Non-parametric analysis was used to determine power and sample size based on ranks (as non-parametric methods may be used to analyze 
subsequent data sets). This demonstrated increased power in the preliminary data $(70-85 \%)$.

\section{Assay quality and detectability}

Twenty-nine different cytokines and chemokines were measured in the cell culture supernatant using a magnetic-bead based protein assay. Each of the 96 wells tested had over 50 beads per cytokine, exceeding the minimal count required for assay reliability. Two sets of quality controls were used to measure expected levels for each of the 29 parameters, which all fell within the expected range. Samples were run in duplicate. IL-3 levels were below the lower limits of detection; IL- 6 and MIP-1 $\alpha$ concentrations were above the upper limits of detection and could not accurately be interpreted. IL-6 was therefore run separately as an individual ELISA. The experimental design is illustrated in Figure 38. 
Table 5. Power and sample size calculations for the multiplex cytokine analysis.

Table 5A.

\begin{tabular}{|c|c|c|c|c|c|c|c|c|}
\hline Target & $\begin{array}{l}\text { Sample } \\
\text { size }\end{array}$ & $\begin{array}{c}\text { Naïve } \\
\text { Mean } \\
\text { (pg/mL) }\end{array}$ & $\begin{array}{l}\text { Impaired } \\
\text { Mean } \\
(\mathrm{pg} / \mathrm{mL})\end{array}$ & $\begin{array}{c}\text { Mean } \\
\text { Difference } \\
(\mathrm{pg} / \mathrm{mL})\end{array}$ & $\begin{array}{l}\text { S.D. of } \\
\text { Difference } \\
\text { (pg/mL) }\end{array}$ & $\begin{array}{l}\text { Effect } \\
\text { size } \\
\text { (d) }\end{array}$ & $\begin{array}{l}\text { Power } \\
(1-\beta)\end{array}$ & $\begin{array}{l}\text { Power* } \\
\text { (ranks) }\end{array}$ \\
\hline TNF- $\alpha$ & 7 & 508.1 & 301.7 & 206.4 & 257.8 & 0.803 & 0.42 & 0.70 \\
\hline IL-10 & 7 & 981.5 & 586.8 & 394.7 & 380.1 & 1.038 & 0.63 & 0.85 \\
\hline IL-6 & 7 & 6659.1 & 3545.7 & 3113.4 & 3228.7 & 0.964 & 0.57 & 0.85 \\
\hline
\end{tabular}

${ }^{*}$ Non-parametric power calculation based on Wilcoxon signed rank test \& subsequent Z-test on ranks.

Table 5B.

\begin{tabular}{|c|c|c|c|c|c|}
\hline$\alpha$ & $\begin{array}{l}\text { Power } \\
(1-\beta)\end{array}$ & $\begin{array}{c}\text { Difference } \\
\text { to detect } \\
\text { (pg/mL) }\end{array}$ & $\begin{array}{l}\text { Effect } \\
\text { size } \\
\text { (d) }\end{array}$ & $\begin{array}{l}\text { Sample } \\
\text { size } \\
\text { required }\end{array}$ & $\begin{array}{l}\text { Sample } \\
\text { size } \\
\text { required* }\end{array}$ \\
\hline \multicolumn{6}{|l|}{ TNF- $\alpha$} \\
\hline 0.05 & 0.80 & If 100: & 0.388 & 55 & \multirow{3}{*}{11.4} \\
\hline 0.05 & 0.80 & If 200: & 0.826 & 16 & \\
\hline 0.05 & 0.80 & If $300:$ & 1.164 & 8 & \\
\hline \multicolumn{6}{|l|}{ IL-10 } \\
\hline 0.05 & 0.80 & If 200: & 0.526 & 31 & \multirow{3}{*}{4.6} \\
\hline 0.05 & 0.80 & If 400: & 1.052 & 10 & \\
\hline 0.05 & 0.80 & If 600: & 1.578 & 6 & \\
\hline \multicolumn{6}{|l|}{ IL-6 } \\
\hline 0.05 & 0.80 & If $1500:$ & 0.464 & 39 & \multirow{3}{*}{4.6} \\
\hline 0.05 & 0.80 & If $3000:$ & 0.929 & 12 & \\
\hline 0.05 & 0.80 & If 4500: & 1.393 & 7 & \\
\hline
\end{tabular}

${ }^{\star}$ Non-parametric estimate of sample size based on noncentrality parameter and expected differences from ranks.

Preliminary data of differences in TNF- $\alpha, I L-10$ and IL-6 between naïve and impaired monocytes at after the $100 \mathrm{ng} / \mathrm{mL}$ LPS challenge (12 hours) is shown in Table 5A. The effect size from the preliminary experiments allows for a sample size calculation, depending on how much of a difference is detectable (Table 5B). Ten donors were used for the subsequent experiment. 
Figure 37. Power-sample size graph based on IL-10 differences between naïve and impaired monocytes at 12 hours following $100 \mathrm{ng} / \mathrm{mL}$ LPS challenge.

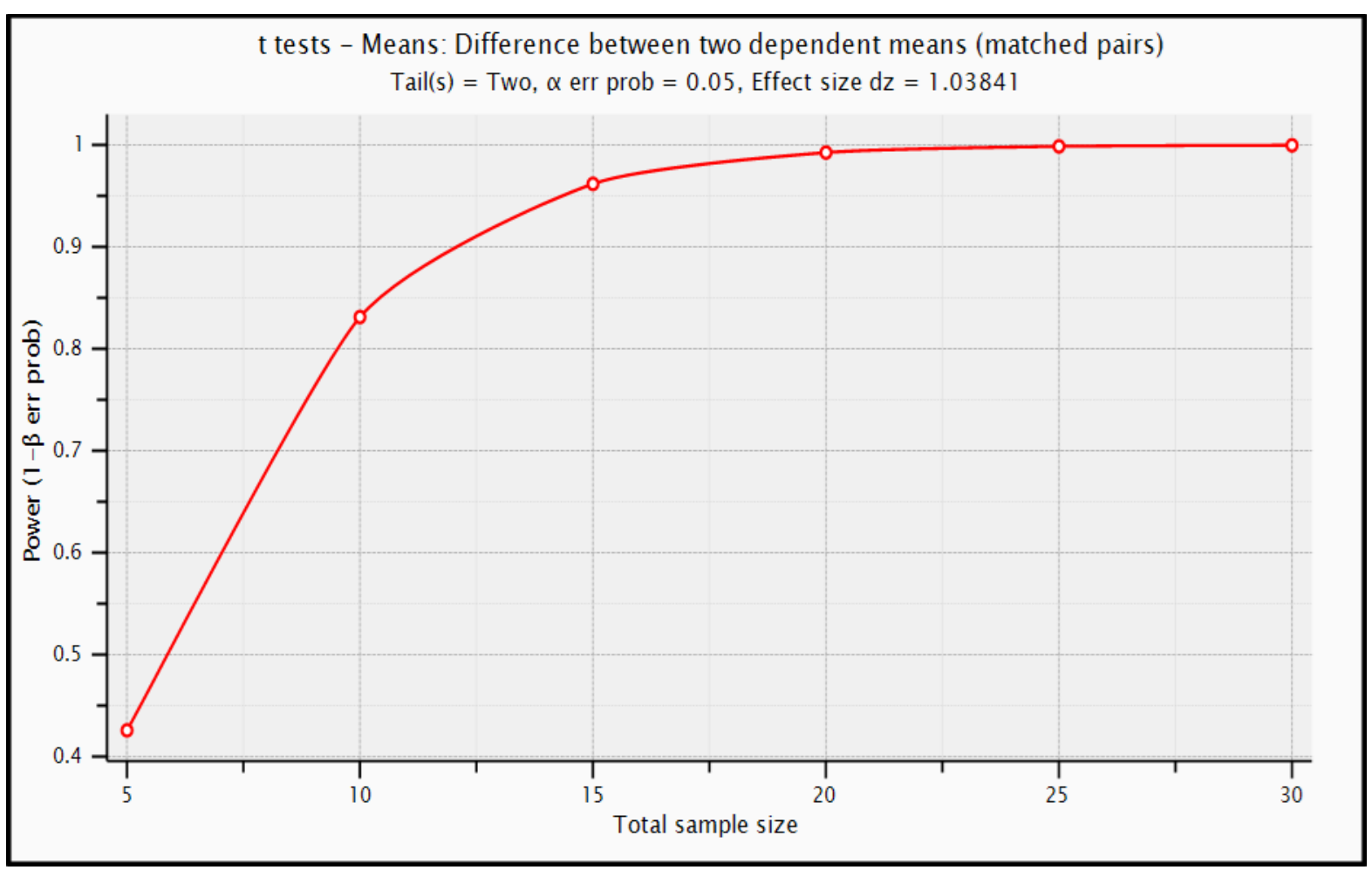

Sample size calculations were plotted against power. This representative plot demonstrates the sample size for IL-10 at 12 hours following an LPS challenge, comparing naïve and impaired monocytes. Note that as the sample size increases, the power of the test obviously increases. A sample size of 10 donors would allow for an experiment to have a power of over 0.8 (80\%).

A similar curve was created for non-parametric sample size calculations (not shown). 
Figure 38. Experimental design of Multiplex cytokine experiments.

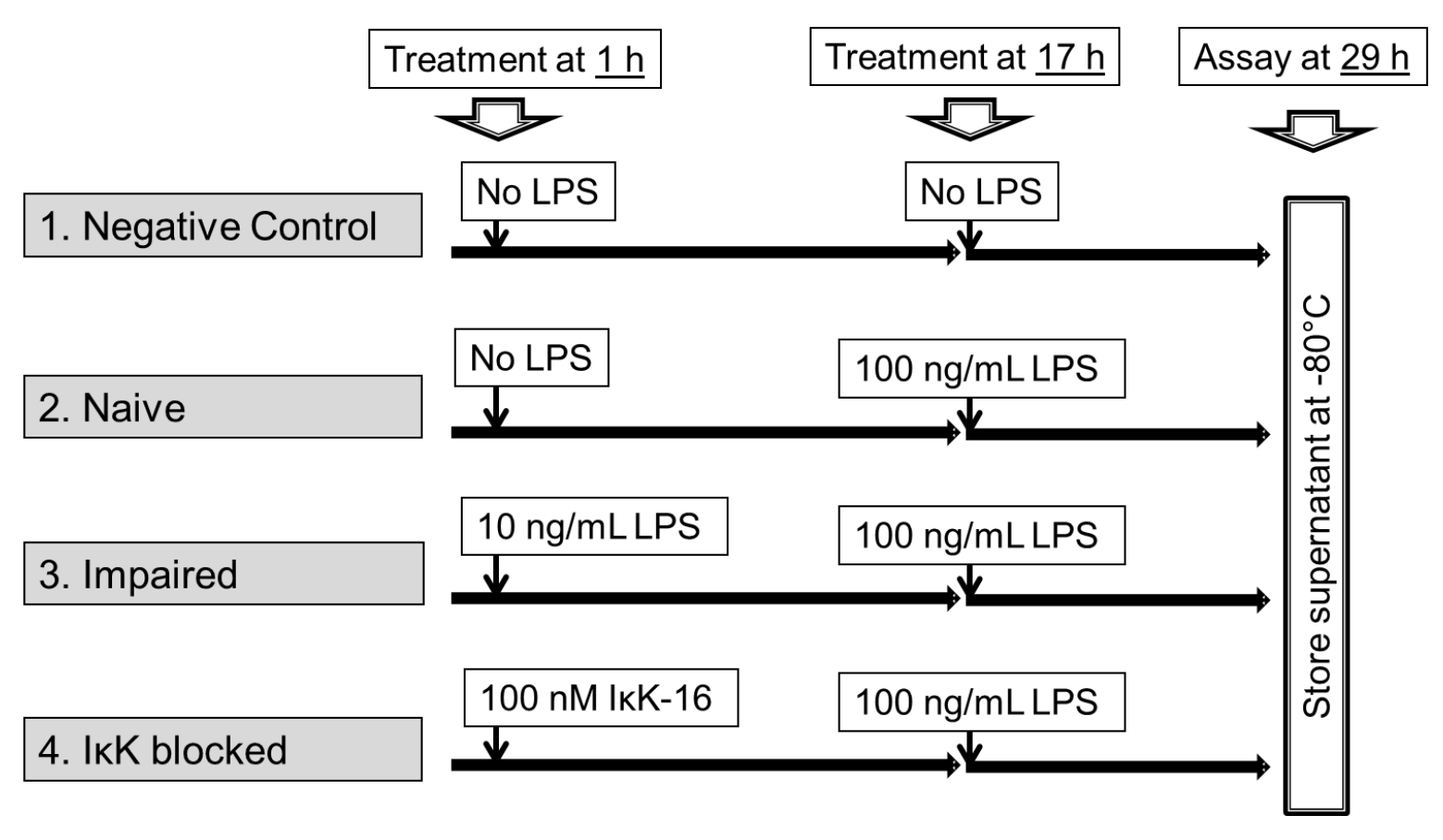

Monocytes were cultured in 4 conditions. In the first 17 hours, cells were treated either with media only (1, unstimulated negative control and 2, naive), low dose $10 \mathrm{ng} / \mathrm{mL}$ LPS (3, impaired) or with $100 \mathrm{nM}$ IKK-16 (4, IKK inhibited) all given at 1 hour after isolation. After 17 hours, cells were resuspended in fresh media and were cultured either with media only (1, unstimulated negative control) or $100 \mathrm{ng} / \mathrm{mL}$ LPS challenge (2, naïve, 3, impaired and 4, IKK inhibited monocytes). At 29 hours (12 hours after $100 \mathrm{ng} / \mathrm{mL}$ LPS challenge), concentrations of cytokines and chemokines in cell culture supernatants were determined by a Milliplex Human Cytokine/Chemokine 29plex magnetic bead assay. 


\section{RESULTS}

Primary Analysis

\section{Pro-inflammatory cytokines}

In response to the $100 \mathrm{ng} / \mathrm{mL}$ LPS challenge, naïve monocytes produced higher levels of TNF- $\alpha, \mathrm{IL}-1 \beta, \mathrm{IL}-6$ and IL-12 compared to the unstimulated control (Figure 39). There were no increases in IL-8 production following LPS treatment. Impaired monocytes, which had prior stimulation with low dose 10 ng/mL LPS, had a decreased capacity to produce TNF- $\alpha$, IL-1 $\beta$ and IL-6 compared to the naïve control. There was also small reduction in IL-12p70 production by impaired monocytes.

IKK inhibition by IKK-16 treatment led to a decrease in TNF-a, IL-12 and IL-8, with profound suppression of IL-1 $1 \beta$ and IL-6 levels compared to naïve cells.

One should note that IL-6 levels higher than the upper limits of detection of the Milliplex assay were observed, and were therefore then separately tested by individual IL-6 ELISA at an appropriately higher dilution and similarly analyzed.

\section{Anti-inflammatory cytokines}

Levels of the anti-inflammatory cytokines IL-1RA and IL-10 were increased with LPS stimulation in the naïve cells compared to the unstimulated negative control, whereas levels of IL-4 and IL-13 were unchanged (Figure 40). Monocyte impairment led to decreases in IL-10 production, but IL-1RA, IL-4 and IL-13 levels were similar between naïve and impaired conditions.

Inhibition of IKK decreased the levels of IL-10, IL-4 and IL-13. Levels of IL1RA were similar between naïve and IkK-16 treated monocytes. 
Figure 39. Effects of monocyte impairment and IKK inhibition on proinflammatory cytokine production.

$$
\mathrm{N}=10 \text { for all }
$$
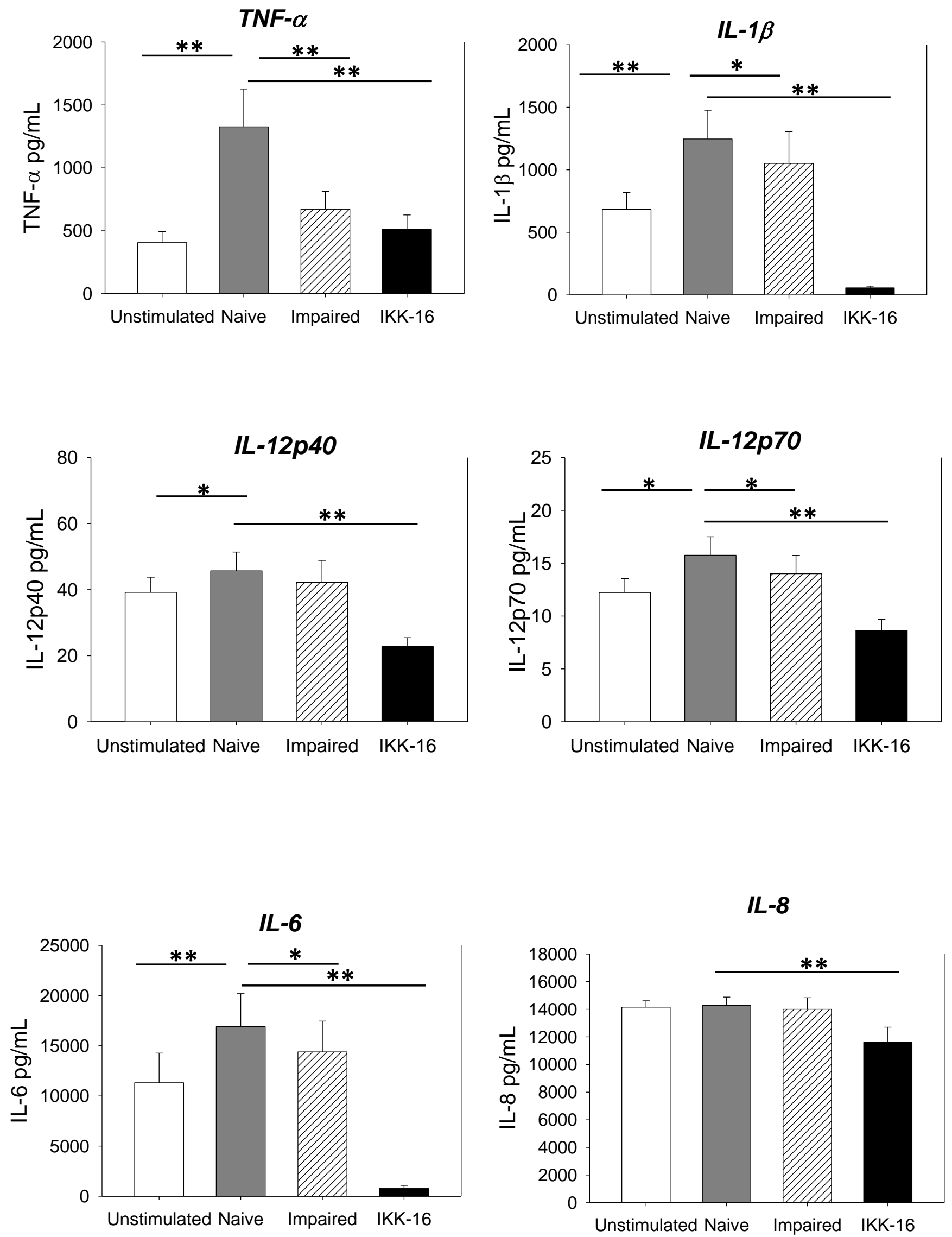


\section{Legend for Figure 39.}

Monocytes were cultured in either media only, low dose $10 \mathrm{ng} / \mathrm{mL}$ LPS or IKK16 (100 nM) for 16 hours. Cells then either remained unstimulated (Unstimulated), or received 100 ng/mL LPS challenge (Naïve, Impaired, IKK16) for 12 hours after the LPS challenge. Cell culture supernatants were measured using a Human Cytokine/Chemokine Milliplex assay. Results for pro-inflammatory cytokines are shown using 10 donors, compared using Wilcoxon Signed rank Test. ${ }^{*} p<0.05,{ }^{* *} p<0.01$. Please note IL-6 was run separately by individual ELISA due to high levels observed in the Milliplex assay. 
Figure 40. Effect of monocyte impairment and IkK inhibition on antiinflammatory cytokine production.
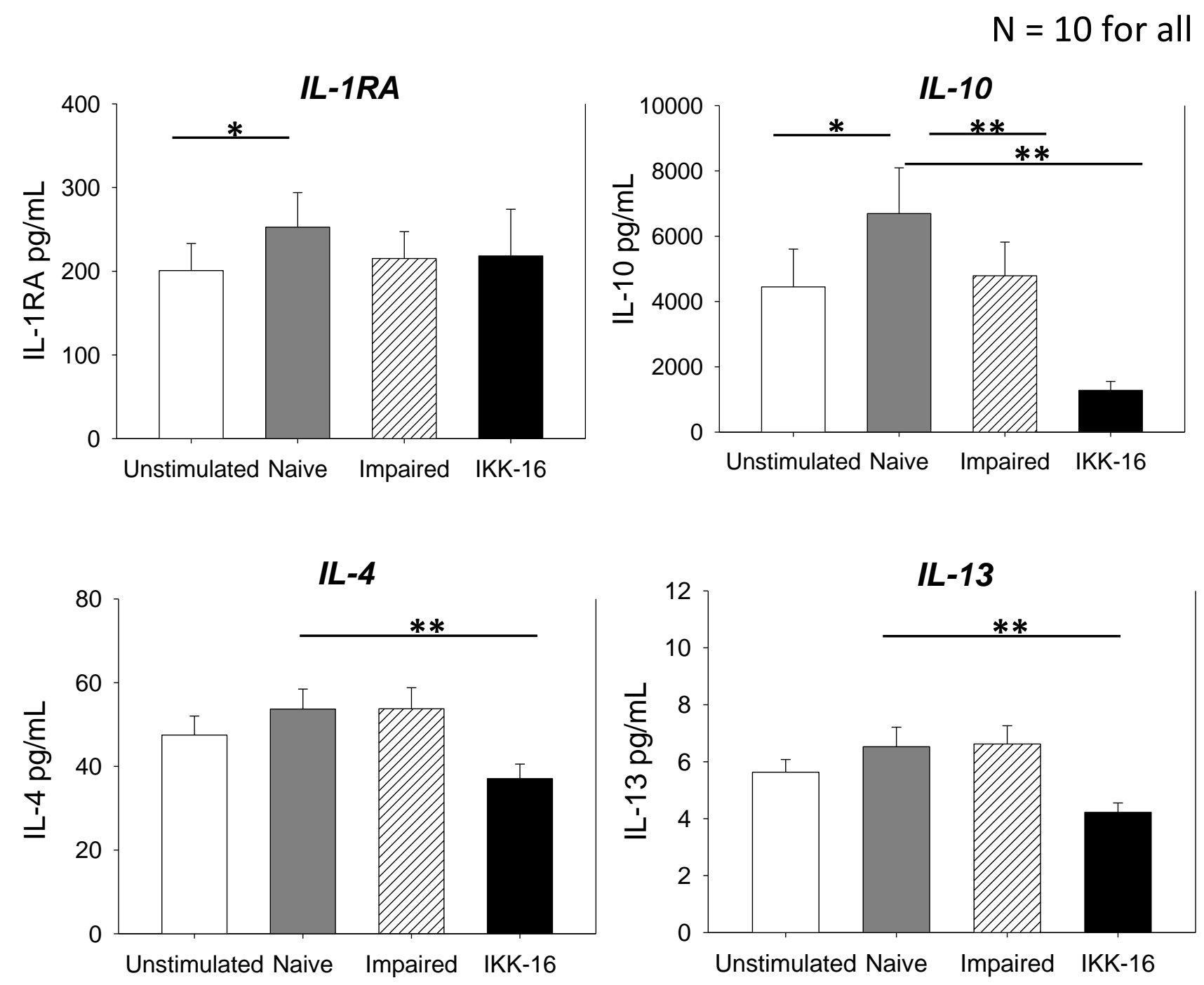

Monocytes were cultured in either media only, low dose $10 \mathrm{ng} / \mathrm{mL} L P S$ or $/ \mathrm{KK}$ $16(100 \mathrm{nM})$ for 16 hours. Cells then either remained unstimulated (Unstimulated), or received $100 \mathrm{ng} / \mathrm{mL}$ LPS challenge (Naïve, Impaired, IKK16) for 12 hours after the LPS challenge. Cell culture supernatants were measured using a Human Cytokine/Chemokine Milliplex assay. Results for anti-inflammatory cytokines are show using 10 donors, compared using Wilcoxon Signed rank Test. ${ }^{*} p<0.05,{ }^{* *} p<0.01$. 


\section{Chemokines}

MIP-1 $\beta$ levels increased in response to LPS in naïve cells, with a very small increase in the levels of another chemokine called eotaxin (Figure 41). IP-10 levels were not increased by LPS stimulation and, interestingly, MCP-1 levels actually decreased slightly compared to unstimulated monocytes.

$10 \mathrm{ng} / \mathrm{mL}$ LPS treatment resulted in suppressed MIP-1 $\beta$, MCP-1 and IP-10 levels in response to the $100 \mathrm{ng} / \mathrm{mL}$ LPS challenge, with eotaxin levels unchanged in impaired cells compared with naive.

IKK-16 treatment decreased the levels of MIP-1 $\beta$, MCP-1 and eotaxin compared to naïve monocytes. Unexpectedly, monocytes with IKK inhibition actually increased IP-10 production by approximately ten fold in response to the $100 \mathrm{ng} / \mathrm{mL}$ LPS challenge compared with naïve monocytes.

MIP-1a levels were above the upper limits of detection of the Milliplex assay and therefore were excluded from analysis.

\section{Growth Factors}

Naïve monocytes produced higher levels of G-CSF, GM-CSF and also VEGF in response to $100 \mathrm{ng} / \mathrm{mL}$ LPS challenge, compared to unstimulated control cells. In monocytes with low dose $10 \mathrm{ng} / \mathrm{mL}$ LPS impairment, the capacity to produce GM-CSF, and to a degree VEGF, was reduced. Impaired monocytes could produce equivalent concentrations of G-CSF and EGF.

IKK-16 treatment decreased the production of all four of these growth factors, but G-CSF and GM-CSF were profoundly decreased (Figure 42). 
Figure 41. Effects of monocyte impairment and IkK inhibition on chemokine production.
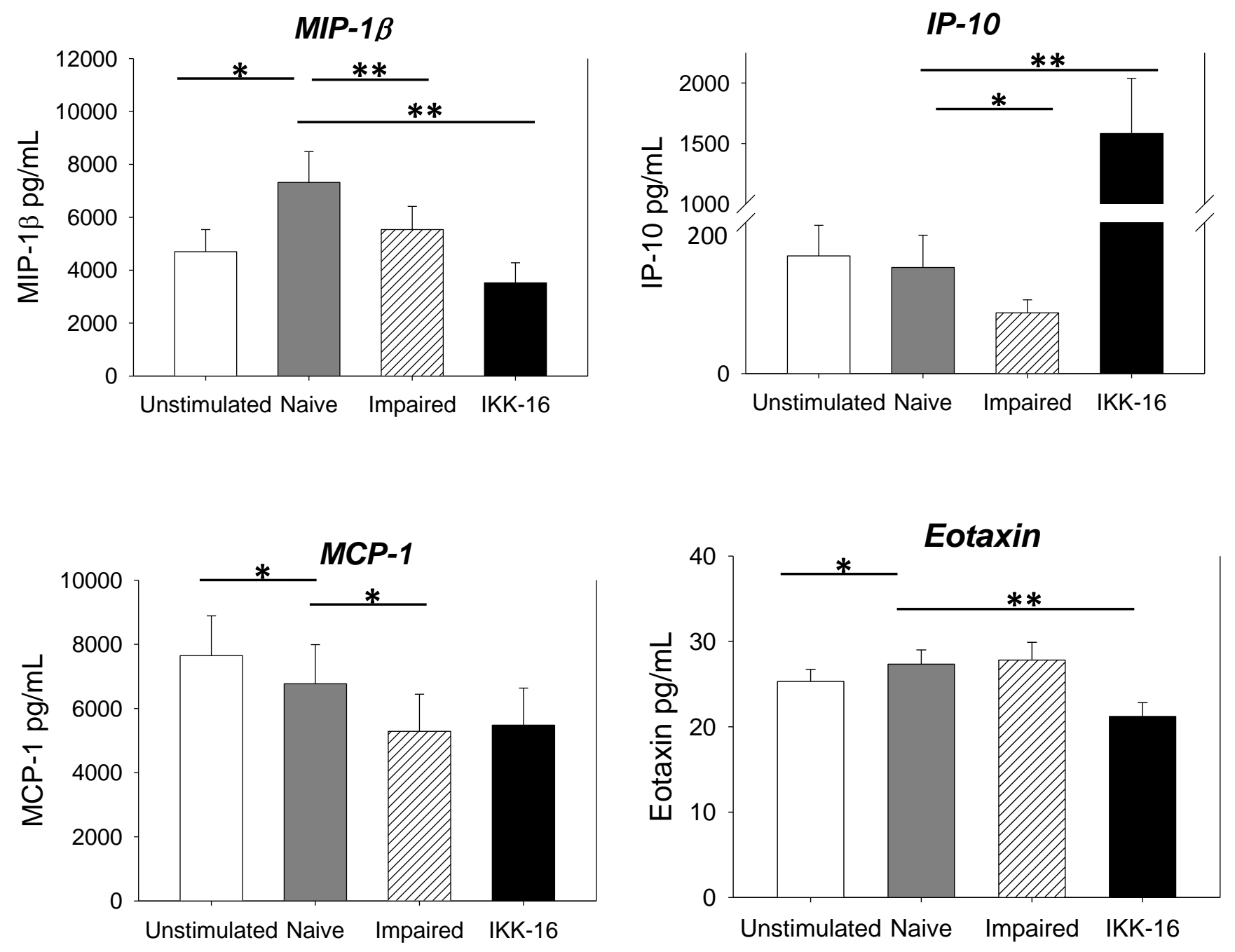

Monocytes were cultured in either media only, low dose $10 \mathrm{ng} / \mathrm{mL}$ LPS or IKK$16(100 \mathrm{nM})$ for 16 hours. Cells then either remained unstimulated (Unstimulated), or received the $100 \mathrm{ng} / \mathrm{mL}$ LPS challenge (Naïve, Impaired, IKK-16) for 12 hours. Cell culture supernatants were measured using a Human Cytokine/Chemokine Milliplex assay. Results for chemokines are shown using 10 donors, compared using Wilcoxon Signed rank Test. * $p$ $<0.05,{ }^{* *} p<0.01$. 
Figure 42. Effect of monocyte impairment and IKK inhibition on growth factor production.
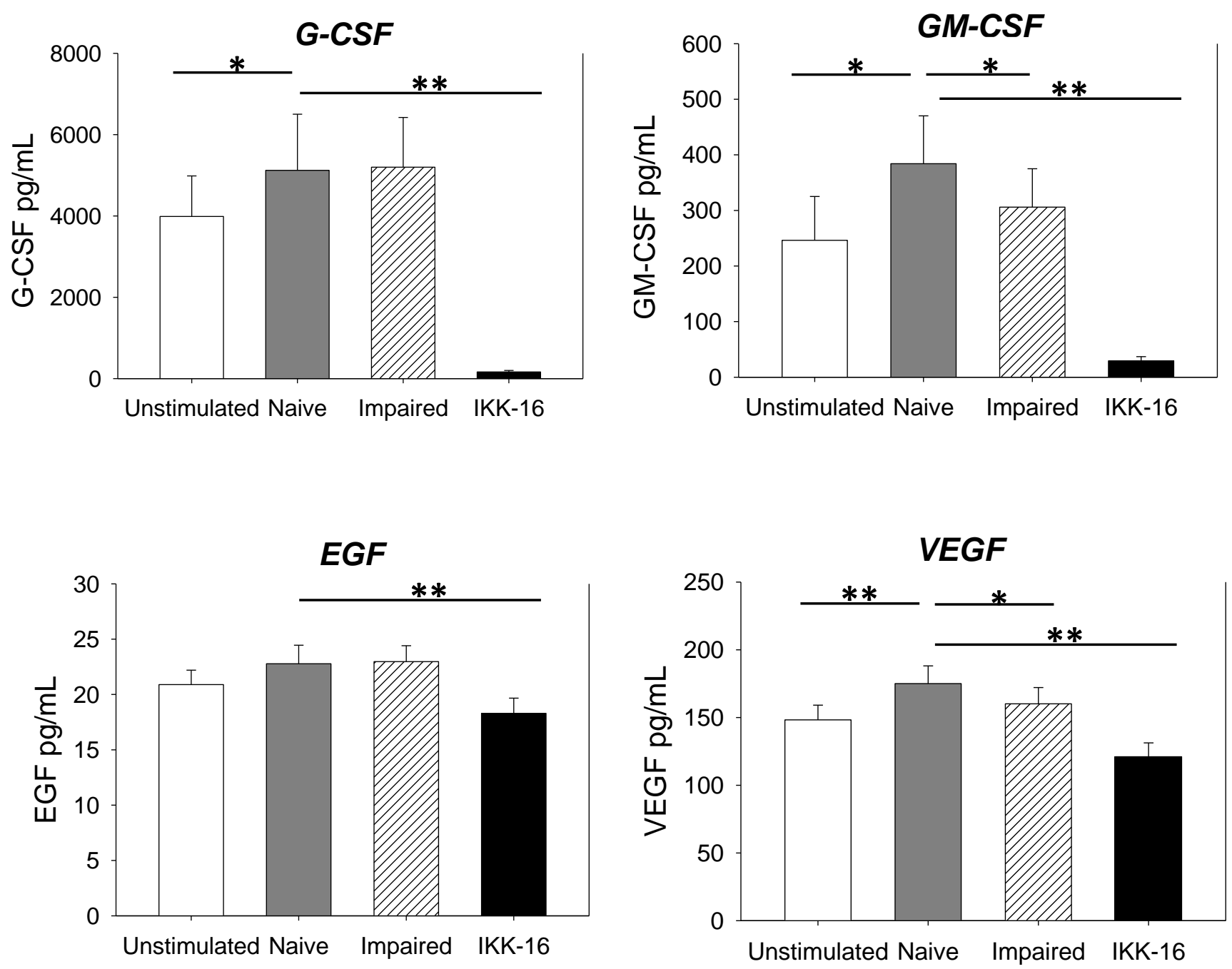

Monocytes were cultured in either media only, low dose $10 \mathrm{ng} / \mathrm{mL} L P S$ or $/ \mathrm{KK}$ 16 (100 nM) for 16 hours. Cells then either remained unstimulated (Unstimulated), or received $100 \mathrm{ng} / \mathrm{mL}$ LPS challenge (Naïve, Impaired, IKK16) for 12 hours after the LPS challenge. Cell culture supernatants were measured using a Human Cytokine/Chemokine Milliplex assay. Results for growth factors are shown using 10 donors, compared using Wilcoxon Signed rank Test. ${ }^{*} p<0.05,{ }^{* *} p<0.01$. 


\section{Other immunoregulatory cytokines}

The $100 \mathrm{ng} / \mathrm{mL}$ LPS challenge increased the levels of IFN- $\alpha 2$, IL-2 and IL-7, compared to unstimulated cells. Impaired monocytes produced decreased levels of IFN- $\alpha 2$, IL-7 and TNF- $\beta$ compared to naïve cells, whereas IFN- $\gamma$, IL15, IL-17 and IL-2 were unchanged between these naïve and impaired conditions. Interestingly, IL-1a was the only cytokine or chemokine tested which was elevated in the impaired cells compared to naïve conditions. IKK inhibition led to a reduction in IFN- $\alpha 2$, IFN- $\gamma$, IL-15, IL-17, IL-1 $\alpha$, IL-2, IL-7 and TNF- $\beta$ levels compared to naïve monocytes (Figure 43). It should be noted that in all four conditions (unstimulated, naïve, impaired and IkK-16 treated), levels of IL-15, IL-17 and IL-2 were detectable but below 10 pg/mL. IL-5 levels were below the minimal detectable concentration of $3.2 \mathrm{pg} / \mathrm{mL}$, and IL-3 levels were completely undetectable. Results for all cytokines/chemokines are summarized for reference in Table 6. 
Figure 43. Effect of monocyte impairment and IKK inhibition on other immunoregulatory cytokines.
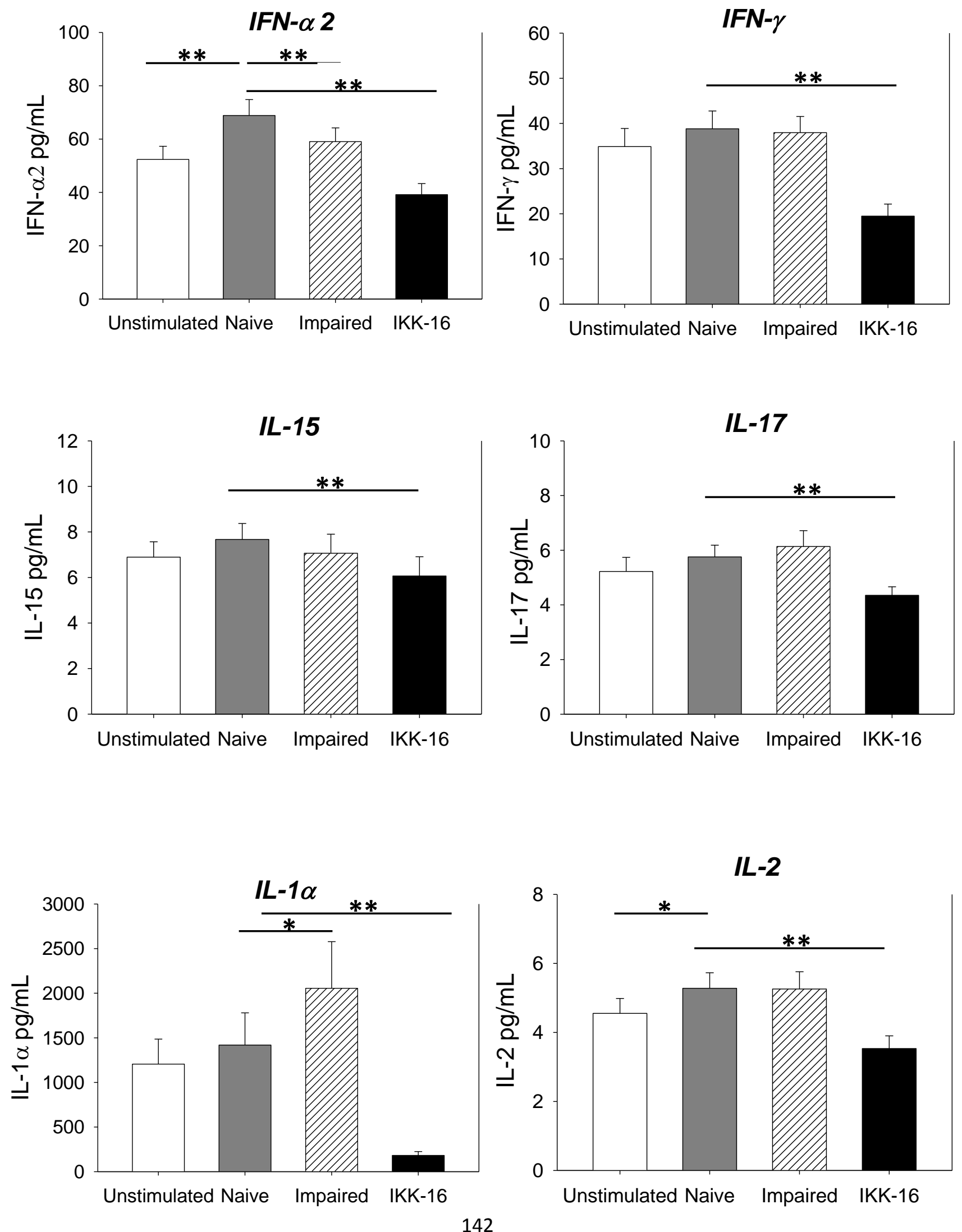


$$
N=10 \text { for all }
$$

TNF- $\beta$

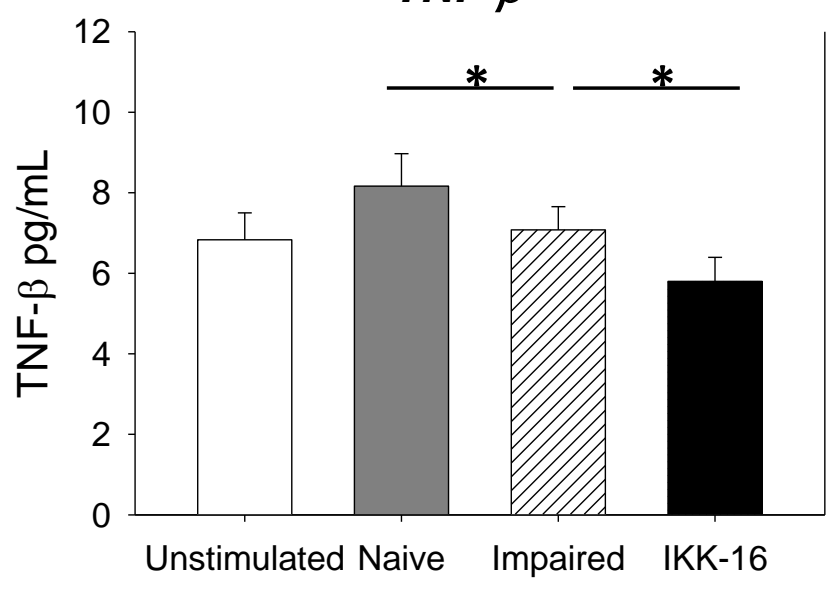

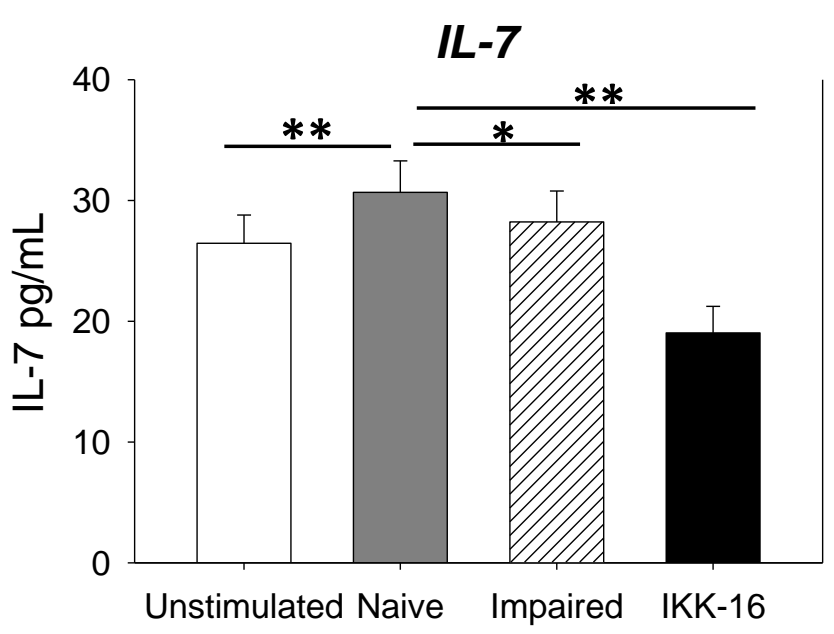

Monocytes were cultured in either media only, low dose $10 \mathrm{ng} / \mathrm{mL} L P S$ or $1 \mathrm{KK}$ 16 (100 nM) for 16 hours. Cells then either remained unstimulated (Unstimulated), or received $100 \mathrm{ng} / \mathrm{mL}$ LPS challenge (Naïve, Impaired, IKK16) for 12 hours after the LPS challenge. Cell culture supernatants were measured using a Human Cytokine/Chemokine Milliplex assay. Results for other immunoregulatory cytokines are shown using 10 donors, compared using Wilcoxon Signed rank Test. * $p<0.05,{ }^{* *} p<0.01$. 


\section{Secondary Analysis}

An additional, more complex statistical analysis was undertaken on the Milliplex cytokine/chemokine data. Given the large variability from donor to donor for a given protein and the large variability between the actual raw levels from protein to protein, this analysis was designed to address these issues to allow cytokine patterns to be compared against one another.

In this analysis, the primary endpoint was the within-subject protein relative change from the Naïve condition to the Impaired condition. Relative change within subject samples was defined as: $\frac{\text { Protein abundance at Impaired Condition }}{\text { Protein abundance at Naive Condition }}$. To ensure the endpoint was approximately normally distributed, the endpoint was $\log$ (base 2) transformed. Student's t-test were then performed with the null hypothesis that the average log relative change was equal to 0 (no difference across condition) versus the alternative hypothesis that average log relative change was not equal to 0 . As multiple comparisons (proteins) were made, qvalues for preserving the False Discovery Rate (FDR) as opposed to the familywise error rate (FWER) are reported. The FDR is defined as FDR = $E\left(\frac{V}{R} \mid R>0\right)$ where $E()$ represents expected value, $V$ is the number of Type I errors (false positives) and $\mathrm{R}$ is the number of rejected null hypotheses ${ }^{121}$. As an example, if differences with $\mathrm{q}<0.05$ are deemed "significant" then on average $5 \%$ of these tests are false positives. Q-values were computed using the method developed by Storey ${ }^{122}$.

This data is shown in Figure $\mathbf{4 4}$ and demonstrates that TNF- $\alpha$ was the most diminished of all cytokines and chemokines measured, and appeared to be the most sensitive supernatant marker measured which reflects the changes 
resulting from monocyte impairment. In addition, the findings demonstrate that numerous chemokines (green) which are significantly downregulated (i.e. near the bottom of the plot), including IP-10, MCP-1 and MIP-1 $\beta$. 
Figure 44. Relative change of cytokine \& chemokine production in the impaired monocyte compared to the naïve cells (Secondary analysis).

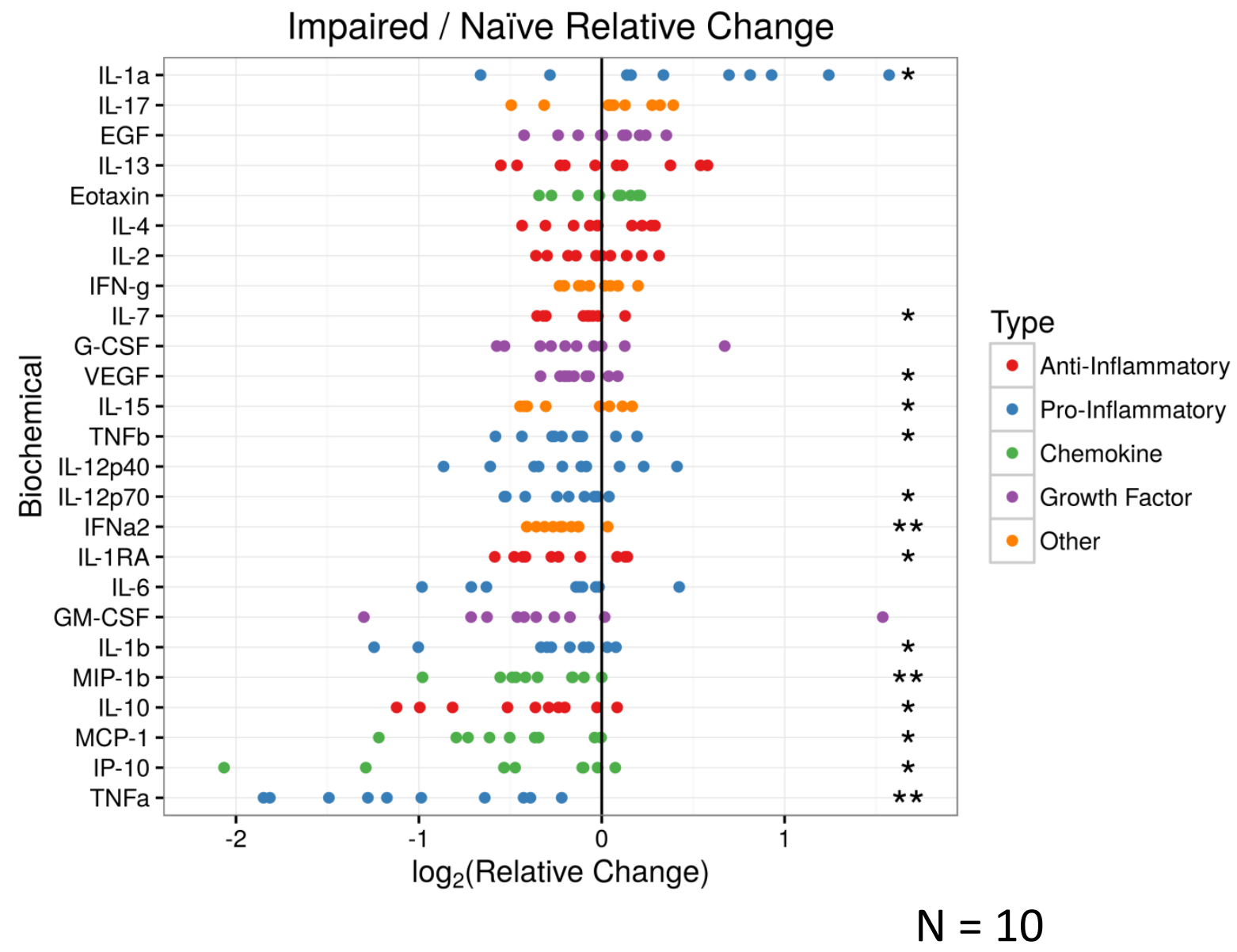

The relative changes of each cytokine/chemokine from naïve to impaired monocytes are summarized here. Each dot represents a within-subject relative change. Stars denote statistical significance. ${ }^{*}: q<0.05^{* *}: q<0.01$. (Q-values are a measure of significance reflecting the false positive rate that accounts for multiple comparisons). Colors denote the function of each protein (e.g. pro-inflammatory). Proteins are ordered top to bottom based on the relative change in the impaired monocyte, from most increased cytokine in the impaired (IL-1 $\alpha$ ) down to the most decreased cytokine at the bottom (TNF- $\alpha$ ).

Secondary analysis was with undertaken with the help of $P$. Trainor (PhD candidate, Statistics). 


\section{DISCUSSION}

A critical balance is required to ensure a sufficient innate response to prevent as well as clear infective challenges to the body, but also to limit an excessive systemic inflammatory response to prevent ongoing damage and tissue destruction. In the context of critical illness in the surgical patient, this balance is often lost. Too much or too little of any aspect of the immunological system has the potential for undesirable consequences. For that reason, the concept of immune modulation carries a risk; "turning off" the immune system following trauma or during sepsis risks nosocomial infection and failed pathogen clearance, and "amplifying" the immune response could risk unnecessary fever and systemic inflammation. This chapter deals with two of these considerations; 1) during conditions of monocyte impairment resulting from prior low dose endotoxin stimulation; which aspects of cytokine and chemokine production are deficient, and which are preserved? 2) Focused, specific inhibition of IKK may decrease TNF- $\alpha$ production but what other cytokines and chemokines are dependent on this pathway? How similar are these two phenomena?

The present experiments were designed with sample size calculations based upon prior pilot experimental data. Ensuring a study is powered sufficiently should reduce the risk of type II error and missing a potentially significant result. Although these calculations were based on TNF- $\alpha$, IL-10 and IL-6 from prior to the experiments, we cannot guarantee that all cytokines assessed would be adequately powered. All experiments were based on 10 donors. 
Endotoxin stimulation of naïve monocytes resulted in a measurable increase in over 15 inflammatory mediators, which included pro-inflammatory and anti-inflammatory cytokines, as well as numerous chemokines. Some of these, such as IL-2, IL-15 and IL-17 were low and although above the lower limits of detection, should be interpreted with caution. By contrast, the levels of production of IL-6 and MIP-1 $\alpha$ by naïve monocytes were above the upper limits of detection and therefore were excluded for analysis. IL-6 was consequently tested separately by individual ELISA, and it is this IL-6 data which is shown. In addition, whilst IL- 8 was within range, I believe that these should also be interpreted with caution as the unstimulated, naïve and impaired conditions were similar at $14,000 \mathrm{pg} / \mathrm{mL}$. It is possible that LPS stimulation increased IL-8 levels beyond that of the unstimulated cells, and levels from the impaired monocyte may be lower than naïve levels, which may not be reflected in our results. It should be noted that similar to previous experiments, the unstimulated monocytes have relatively high levels of many inflammatory mediators which is likely a function of cellular stimulation from the isolation procedure and centrifugation steps.

Prior results in this dissertation have indicated that TNF- $\alpha$, IL-6 and IL10 were important inflammatory cytokines that were suppressed in the impaired monocyte. In this different assay platform, TNF- $\alpha$, IL-10 and IL-6 were again all decreased compared with naïve cells, which confirms the previous results. These data show the impaired monocyte also has a

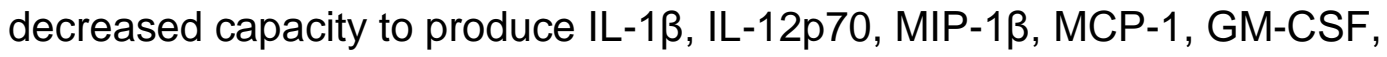
VEGF, IFN- $\alpha 2$, TNF- $\beta$ and IL-7. The identification of these inflammatory mediators as suppressed in this model suggests that both chemokine and 
growth factor production by the monocyte are functions that are also to some degree impaired. This would add further explanation to the decreased pathogen clearance seen in such patients, due to decreased leukocyte chemoattraction, as well as the lack of maturation and differentiation of new circulating leukocytes such as myeloid-derived suppressor cells ${ }^{123,124}$. Many pro-inflammatory cytokines are produced in response to NFKB activation, thus a reduction in all of these pro-inflammatory cytokine responses after IKK-16 was expected.

Anti-inflammatory cytokines are important mediators in resolving inflammation after an initial insult, but excess levels can themselves suppress other genes. LPS effectively increases IL-1RA and IL-10, but only IL-10 was suppressed in the impaired monocyte compared to the naïve cells. This supports the notion that global functions were impaired, rather than a polarization of monocytes and macrophages toward an "M2" phenotype, where higher IL-10 levels would be expected. The significance of the decrease in anti-inflammatory cytokines following IKK inhibition (with IKK-16) is that this may "maintain the balance" with the reduced pro-inflammatory cytokines, preventing a dominance of these anti-inflammatory mediators. There are some specific cytokines and chemokines worthy of attention. 
Figure 45. Summary of the effect of monocyte impairment and IkK inhibition on cytokine and chemokine networks (as per the primary analysis).

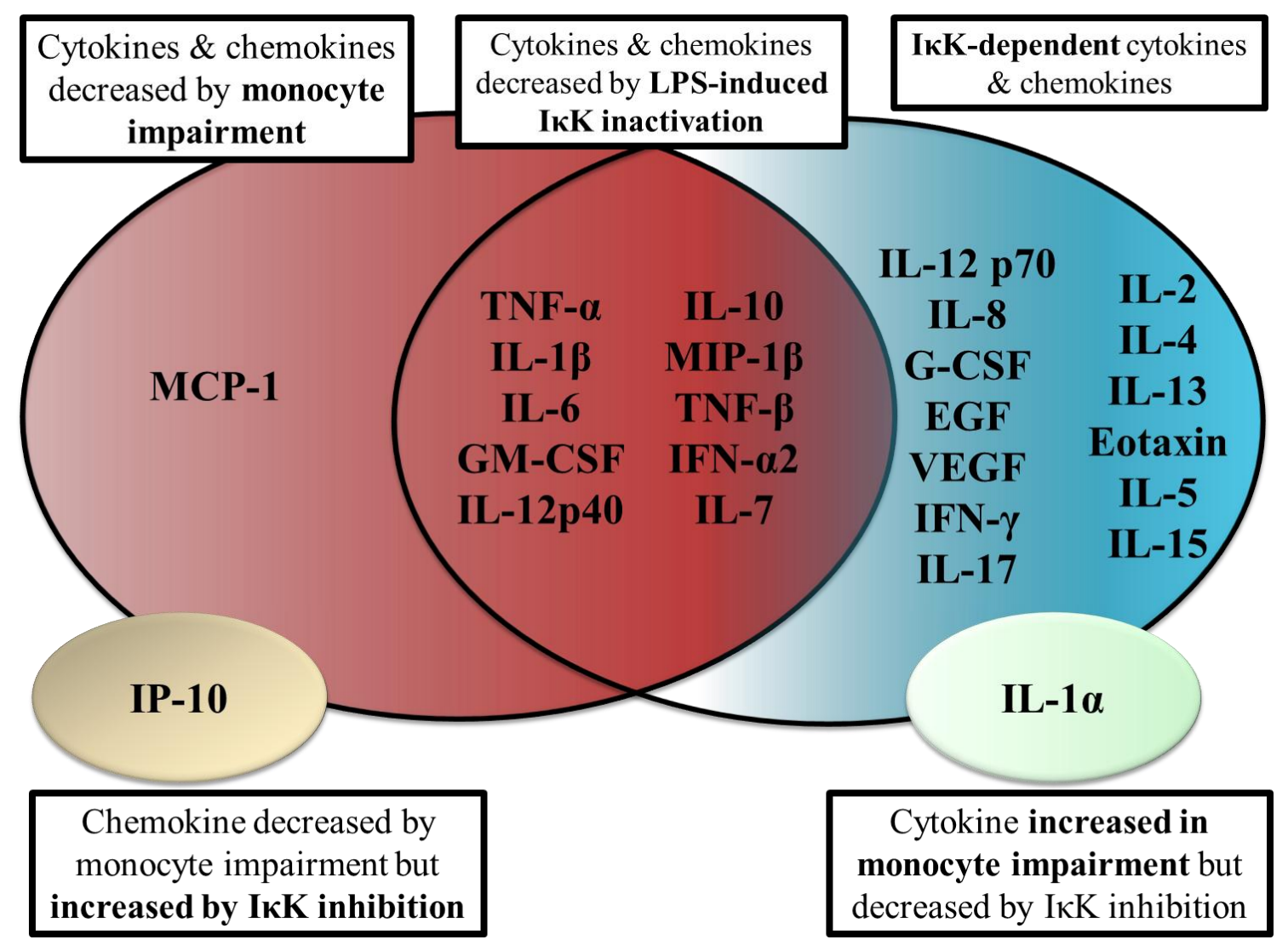

Low dose LPS $10 \mathrm{ng} / \mathrm{mL}$ results in monocytes having an impaired capacity to produce a large number of cytokines and chemokines (red). Specific inhibition of IKK, a central feature of monocyte impairment, results in decreased levels of all cytokines and chemokines seen in blue. Those inflammatory mediators which are decreased in both conditions are shown in the central region where the two networks overlap. Cytokines that were undetectable are not shown. MCP-1 is the only mediator which is decreased in monocyte impairment but not influenced by IKK inhibition. IP-10 is also decreased in monocyte impairment, but increases during specific IKK inhibition. IL-1a was the only mediator which was increased in monocytes impaired by prior $10 \mathrm{ng} / \mathrm{mL} L P S$ treatment, however, does appear to be dependent on IKK function. 
MCP-1, also known as monocyte chemoattractant protein-1 or CCL2, yielded some interesting findings. MCP-1 has previously been described as having a central role in injury-induced inflammation ${ }^{125}$. This was the only mediator to decrease in response to the $100 \mathrm{ng} / \mathrm{mL}$ LPS challenge, and was the only cytokine decreased in the impaired monocyte but not regulated by IKK signaling. MCP-1 is thought to be governed by JNK and MAPK p38 pathways, and therefore MCP-1 suppression may suggest alterations in these pathways ${ }^{126}$. It should be noted that the difference between naïve and IKK-16 treated monocytes was only $p=0.08$.

IP-10 (interferon-gamma induced protein-10), also known as CXCL-10, is generally secreted in response to IFN- $y$ with important roles in chemoattraction for other leukocytes, and is thought to govern IL-10 production. In our results, this mediator showed an unexpected pattern. The LPS challenge did not influence levels in the naïve monocyte relative to unstimulated cells, but levels were decreased in the impaired monocyte. Furthermore, it was the only parameter that consistently increased after IKK inhibition. This may suggest that that other cytokines, either raised or decreased in the first 17 hours, may modulate IP-10 levels rather than directly from LPS stimulation. In addition, there is presumably an intermediate molecule which normally suppresses IP-10, that is blocked by IKK-16 and thus releasing IP-10 production (in other words, "the inhibitor is inhibited"). IP-10 had previously been thought to be NFKB-dependent; however these results suggest that other pathways are involved in its production ${ }^{127}$.

IL-1 $\alpha$ was the only cytokine which significantly increased in the context of monocyte impairment compared to naïve cells. This was an unexpected 
result, and to my knowledge has not been previously associated with monocyte impairment or "endotoxin tolerance". IL-1 $\alpha$ has the capacity to stimulate TNF- $\alpha$, and both are synergistic in their interaction. In these results, IL-1 $\alpha$ upregulation is in contrast to TNF- $\alpha$ and to IL-1 $\beta$, which has a similar biochemical structure. Monocytes have a unique mechanism of inducible IL1a expression through a long non-coding RNA, but some evidence also shows that cell death can lead to the passive leakage of cytosolic IL-1a. Impaired monocytes have appeared to have similar rates of cell viability to naïve cells according to our previous results, although we have not formally measured apoptosis. A more likely explanation is that IL-1 $\alpha$ is produced as a result of the initial $10 \mathrm{ng} / \mathrm{mL}$ LPS stimulus in impaired cells, where its release is persistent in a well-recognized, feed forward, "self-perpetuating" inflammatory loop ${ }^{124}$. This loop appears to be successfully interrupted by IKK inhibition.

Taken together, the results from this set of experiments expand our knowledge regarding the extent of impairment of monocyte function, with a now extended range of inflammatory mediators in which impaired monocytes have decreased capacity to produce and release. The pattern of change in the impaired monocyte highlights those cytokines and chemokines are most affected in impairment, as demonstrated by the secondary analysis (Figure 44). There is significant overlap between impaired monocyte function from prior LPS exposure and monocytes inhibited by an IKK inhibitor, supporting the role of IkK suppression in monocyte impairment (Figure 45). There are a considerable number of mediators which are suppressed by IKK, but are not decreased in the impaired monocyte, which suggests pleiotropic effects of 
LPS as a "sledgehammer" which may result in cross-talk between other inflammatory pathways and redundant feedback loops preserving their production through non-IKK mechanisms. Another important consideration is the potential for "off-target" effects of IKK-16. While it is believed that this inhibitor is specific for IKK, as with any drug, it may have unrecognized effects such as the inhibition or activation of other pathways in the inflammatory response. What is clear from these results is the broad spectrum of inflammatory control governed by IKK activation. The Pittsburgh group have associated the elevation of at least 15 cytokines or chemokines with later nosocomial infection in trauma patients, that we have shown can be decreased by IKK-16 ${ }^{120}$. The magnitude of the increase in pro- and antiinflammatory cytokines and chemokines, as well as the magnitude of the decrease in adaptive immunity genes predicted poor outcomes in trauma patients (Figure 46) ${ }^{1}$. The results presented here demonstrate that IkK-16 has the capacity to attenuate many of these pro- and anti-inflammatory cytokines and chemokines, but crucially without further impairment to HLA-DR expression. This pattern of modulation makes IKK-16 an attractive therapeutic avenue. 
Figure 46. The genomic response to trauma and potential role of IKK inhibition.

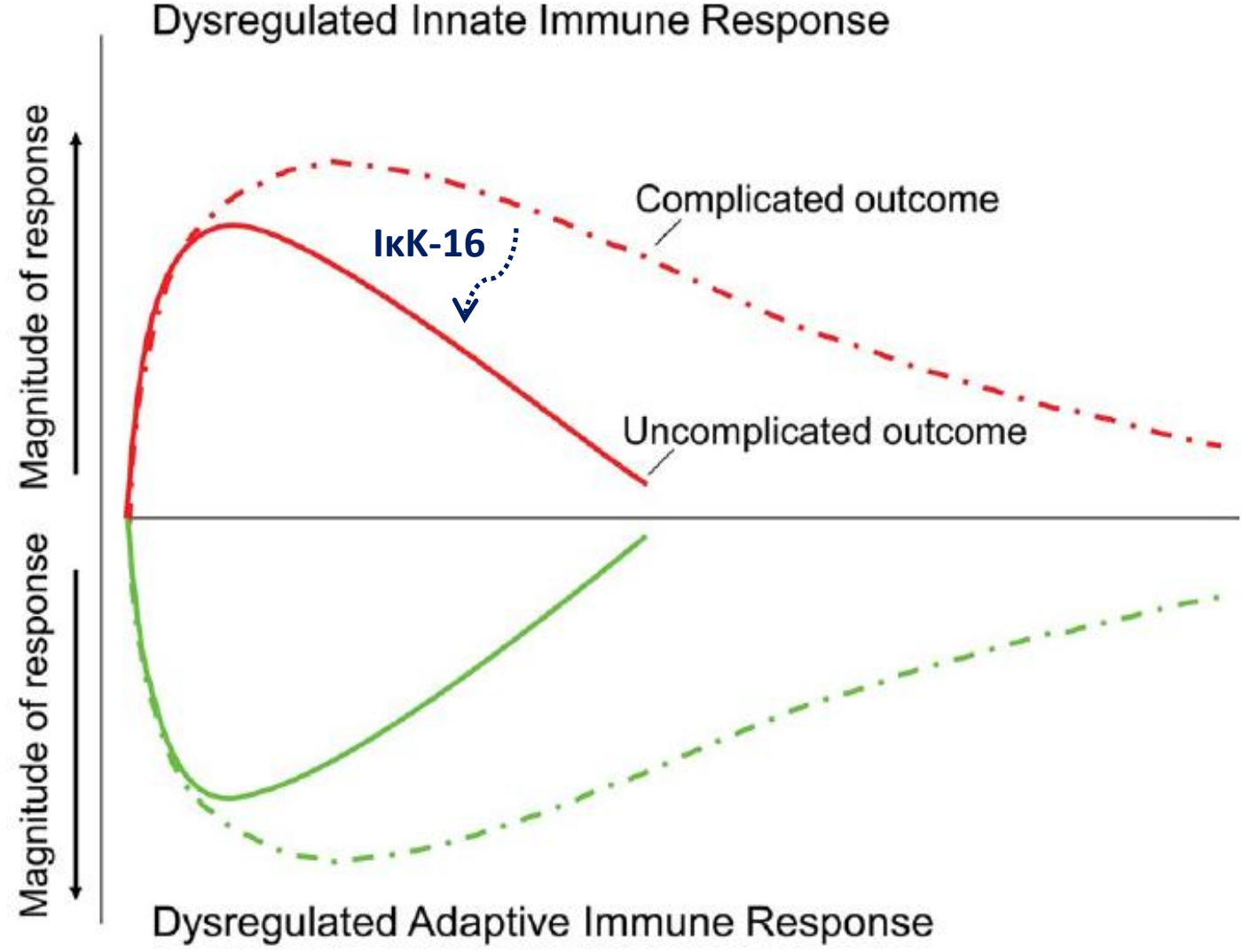

The genomic response to trauma is not changed by a "second hit" but nosocomial infection and mortality are predicted by the magnitude of the change after injury. The larger the increase in innate immunity gene expression (such as pro- and anti-inflammatory cytokines and chemokines) is related to poorer outcomes. In addition, the level of downregulation in adaptive immunity genes such as HLA-DR is also predictive of infection and mortality. Taken together, our results show that IKK-16 attenuates the response in pro- and anti-inflammatory cytokines and chemokines, without a reduction in HLA-DR expression. This pattern of modulation makes IKK inhibition an attractive therapeutic strategy (blue arrow). Adapted from Xaio, 2011 (as part of the Glue Grant studies) ${ }^{1}$. 
Table 6. Effect of monocyte impairment and IkK inhibition on cytokine

production.

\begin{tabular}{|c|c|c|c|c|c|c|c|c|}
\hline & Unstimulated & Naïve & Impaired & IKK-16 & $\begin{array}{c}P(1) \\
\text { Impaired } \\
\text { vs. Naïve }\end{array}$ & $\begin{array}{l}\text { P (1) } \\
\text { IKK-16 } \\
\text { vs. } \\
\text { Naive } \\
\end{array}$ & $\begin{array}{c}\text { P (2) } \\
\text { Impaired } \\
\text { vs. Naïve }\end{array}$ & $\begin{array}{l}P(2) \\
\text { IkK- } \\
16 \text { vs. } \\
\text { Naive }\end{array}$ \\
\hline \multicolumn{9}{|c|}{ Pro-inflammatory cytokines } \\
\hline TNF- $\alpha$ & 405.5 (86.6) & $1325.8(300.6)$ & $671.3(140.4)$ & $509.8(115.6)$ & $\downarrow 0.002$ & $\downarrow 0.002$ & $<0.05$ & $<0.05$ \\
\hline IL-1 $\alpha$ & $1205.4(280.2)$ & $1419.3(360.7)$ & 2054.8 (522.9) & $182.4(42.5)$ & $\uparrow 0.027$ & $\downarrow 0.002$ & NS & $<0.05$ \\
\hline IL-1 $\beta$ & $683.1(134.8)$ & $1245.9(230.0)$ & $1050.5(253.1)$ & $56.08(13.8)$ & $\downarrow 0.049$ & $\downarrow 0.002$ & $<0.05$ & $<0.05$ \\
\hline IL-12p70 & $12.2(1.3)$ & $15.8(1.7)$ & $14.0(1.7)$ & $8.63(1.0)$ & $\downarrow 0.010$ & $\downarrow 0.002$ & NS & $<0.05$ \\
\hline IL-6 & 11310.1 (2949.1) & $16899.2(3288.1)$ & $14384.4(3066.9)$ & 764.6 (314.9) & $\downarrow 0.010$ & $\downarrow 0.002$ & $<0.05$ & $<0.05$ \\
\hline \multicolumn{9}{|c|}{ Anti-inflammatory cytokines } \\
\hline IL-10 & $4446.3(1157.6)$ & 6692.7 (1398.9) & $4785.9(1032.5)$ & $1279.5(272.1)$ & $\downarrow 0.006$ & $\downarrow 0.002$ & $<0.05$ & $<0.05$ \\
\hline IL-1RA & $200.8(32.3)$ & $252.6(41.3)$ & $215.1(32.1)$ & $218.3(55.7)$ & 0.084 & 0.322 & NS & NS \\
\hline \multicolumn{9}{|c|}{ Chemokines } \\
\hline MCP-1 & $7647.6(1238.3)$ & 6770.5 (1219.9) & $5287.8(1156.7)$ & $5482.5(1153.2)$ & $\downarrow 0.002$ & 0.084 & $<0.05$ & NS \\
\hline MIP-1 $\beta$ & $4699.8(834.2)$ & 7318.2 (1104.8) & 5534.1 (835.6) & 3524.4 (755.7) & $\downarrow 0.004$ & $\downarrow 0.004$ & $<0.05$ & $<0.05$ \\
\hline IP-10 & $116.7(30.2)$ & 105.2 (31.9) & $60.2(12.9)$ & $1582.2(455.4)$ & $\downarrow 0.020$ & $\uparrow 0.004$ & $<0.05$ & $<0.05$ \\
\hline Eotaxin & $25.3(1.4)$ & $27.3(1.7)$ & $27.8(2.1)$ & $21.2(1.6)$ & 0.625 & $\downarrow 0.002$ & NS & $<0.05$ \\
\hline IL-8 & $14150.2(462.5)$ & 14283.3 (597.9) & $14000.0(833.5)$ & $11606.2(1097.0)$ & 0.492 & $\downarrow 0.002$ & NS & $<0.05$ \\
\hline \multicolumn{9}{|c|}{ Growth factors } \\
\hline G-CSF & 3987.7 (997.1) & $5121.1(1234.5)$ & $5199.3(1160.4)$ & $162.9(37.8)$ & 0.250 & $\downarrow 0.008$ & NS & $<0.05$ \\
\hline GM-CSF & $246.3(78.8)$ & $384.0(86.2)$ & 305.9 (69.2) & 29.5 (7.5) & $\downarrow 0.027$ & $\downarrow 0.002$ & $<0.05$ & $<0.05$ \\
\hline EGF & $20.9(1.3)$ & $22.8(1.7)$ & $23.0(1.4)$ & $18.3(1.4)$ & 0.846 & $\downarrow 0.002$ & NS & $<0.05$ \\
\hline VEGF & $148.3(10.8)$ & $175.0(13.1)$ & $160.1(12.0)$ & $121.1(13.2)$ & $\downarrow 0.020$ & $\downarrow 0.002$ & $<0.05$ & $<0.05$ \\
\hline \multicolumn{9}{|c|}{ Other immunoregulatory cytokines } \\
\hline IFN- $\alpha 2$ & $52.4(4.9)$ & $68.8(6.0)$ & $59.0(5.1)$ & $39.2(4.1)$ & $\downarrow 0.004$ & $\downarrow 0.002$ & $<0.05$ & $<0.05$ \\
\hline IFN- $\boldsymbol{\gamma}$ & $34.9(4.0)$ & $38.8(3.9)$ & $38.0(3.6)$ & $19.5(2.7)$ & 0.695 & $\downarrow 0.002$ & NS & $<0.05$ \\
\hline IL-4 & $47.5(4.5)$ & $53.7(4.8)$ & $53.7(5.0)$ & $37.0(3.5)$ & 1.000 & $\downarrow 0.002$ & NS & $<0.05$ \\
\hline IL-7 & $26.5(2.3)$ & $30.7(2.6)$ & $28.2(2.6)$ & $19.0(2.2)$ & $\downarrow 0.037$ & $\downarrow 0.002$ & $<0.05$ & $<0.05$ \\
\hline
\end{tabular}

Mean (SEM) shown. P-values (1) reflect Wilcoxon signed rank test. P-values (2) reflect

Repeated Measures ANOVA with Post Hoc Student Newman Keuls test. Arrows reflect the

direction of statistically significant differences in cytokines/chemokines between indicated

comparisons. Cytokines out-with upper and lower detection limits are excluded. IL-6 data

run as individual ELISA as described. 


\section{PATHWAY ANALYSIS}

Pathway analysis was undertaken to interpret the complex pattern of cytokine, chemokine and surface receptor differences between the studied experimental conditions representing monocyte functionality.

Specifically, the MetaCore ${ }^{\mathrm{TM}}$ database (Thomson Reuters) was used to determine signaling networks based on significant cytokines and chemokines ( $p<0.05$ from Repeated Measures ANOVA with Post Hoc Student Newman Keuls test between naïve and impaired monocytes) and HLA-DR (deemed to be significantly different as per Specific Aim 1). The "Build a Network" function was used, by entering the significantly different proteins (seed nodes) based on our data. Then, a network was built and analyzed an algorithm for up to 50 nodes. This yielded 30 networks, which were ranked in order of Zscore. The Z-score ranks the subnetworks according to the saturation of the objects from the original list of seed nodes. The formula to calculate this is as

follows: $\quad$ Z-score $=\frac{r-n \frac{R}{N}}{\sqrt{n\left(\frac{R}{N}\right)\left(1-\frac{R}{N}\right)\left(1-\frac{n-1}{N-1}\right)}}$, where $\mathrm{N}$ is the total number of nodes in the database, $\mathrm{R}$ is the number of network objects corresponding to significant genes (i.e. cytokines, chemokines and surface markers entered), $\mathrm{n}$ is the number of nodes in each small network generated from the output list, and $r$ is the number of nodes with data in each small network from the output list.

The results from this analysis are summarized in Table 7 . The analysis demonstrates that the highest ranking network generated is in fact "NFKB", the signaling pathway that has been the focus of this thesis. This yielded a pvalue of $7.78 \mathrm{e} 46$, with a Z-score of 323.72 . It should be noted that this 
network achieved highest scores in terms of low p-value, high Z-score and high G-score (the G-score is a modified Z-score based on how patterns relate to Canonical Pathways in the database). Processes that this network were associated with, based on the significant targets entered, included "positive regulation of the immune system", "cellular response to LPS", "cellular response to a molecule of bacterial origin", "positive regulation of leukocyte migration" and "leukocyte chemotaxis".

The extent of the role of NFKB in how it relates to defective protein synthesis is shown in Figure 47A. Each arrow (connection) is based on peer reviewed literature evidence for a relationship between two targets, therefore, defective inflammatory NFKB activation observed in the impaired monocyte could explain differences in the linked cytokines and chemokines, but also low HLADR expression in the impaired monocyte relative to the naive.

Interestingly, the third and fourth most correlated networks were RelA (the gene name for $\mathrm{p} 65$, the important active subunit of $\mathrm{NFKB}$, and the NFKB heterodimer p50/p65), respectively. These are all overlapping networks and signify the importance of defective function of NFKB in the impaired monocyte.

A further discussion point is the highly scoring network of AP-1 (also known as Activator Protein-1). AP-1 is a transcription factor which regulates the gene expression of various proteins relating to differentiation, proliferation and apoptosis. It is a heterodimer composed of proteins such as c-Fos, C-Jun and ATF families. C-Jun is also the fifth most commonly involved network. In Figure 48, it is clear that this transcription factor is activated by p38, ERK and JNK, which are the critical upstream inflammatory signaling pathways 
involved in the monocyte inflammatory response. This cross-talk between pathways implies that both NFKB and AP-1 (p38, ERK and JNK) have overlapping roles in mounting a response to an infective stimulus. This again highlights the redundancy of the innate immune response. We have shown that indeed IkK and NFKB are suppressed, and could account for the network of suppressed protein responses seen. However, given that these alternative pathways culminating in AP-1 have not "compensated" for the decreases in NFkB activation, this implies that these pathways are also similarly suppressed.

A further conclusion from this pathway analysis is a potential explanation for the pattern of MCP-1 levels (also known as CCL2) observed. This important chemokine is regulated by both NFKB (As per Figure 47A), but also by AP-1 (Figure 48). Given that MCP-1 was not suppressed by an IKK-16 (a blocker of IKK and subsequently NFKB) in the naïve monocyte, this suggests that MCP-1 production is an AP-1 mediated mechanism (presumably through MAPK p38, ERK or JNK) in the context of monocyte impairment.

We also used the network analysis to consider some other anomalies seen in our data. Firstly, we found that the impaired monocyte actually produced consistently higher levels of IFN- $\alpha 2$ compared with the naïve monocyte. This is in contrast to Shi et al, who found that IFN- $\alpha 2$ was decreased during monocyte impairment, and replenishing IFN- $\alpha 2$ levels with an exogenous source could abrogate this impairment ${ }^{128}$.

Regulation of IFN- $\alpha 2$ production is governed by various mechanisms, including the non-canonical NFKB pathway (involving TRAM-TRIF-TRAF3- 
TBK1//KK-ع) through either interferon regulatory factor (IRF)-3 or IRF-7. Therefore, given that IP-10 is also suppressed in the impaired monocyte but IFN- $\alpha 2$ is elevated, this suggests that opposing effects of IRF function with suppressed IRF-3 and elevated IRF-7 function may be occurring (Figure 48). Through pathway analysis using MetaCore ${ }^{\mathrm{TM}}$, the two most consistent pathways governing HLA-DR expression were NFKB and JAK-STAT1 signaling. The former is demonstrated in Figure 47A, and the latter is shown in Figure 47B. In the impaired monocyte, HLA-DR expression is decreased, which could be explained by deficiencies in either of these pathways. However, when naïve cells were treated with IkK-16, which we know inhibits IKK and NFKB functions, HLA-DR expression is unaffected. This suggests an alternative pathway is being suppressed to cause the deficient HLA-DR expression, and the literature and database pathway analysis is suggestive that the JAK-STAT1 pathway is also suppressed in the impaired monocyte. This is very likely as SOCS-1 (suppressor of cytokine signalling-1) is elevated in impaired monocyte models, and is known to inhibit JAK-STAT1 signaling. In fact, SOCS-1 is required for endotoxin tolerance.

In summary, pathway analysis reveals that the most consistent signaling network corresponding to the data generated from our experiments is NFKB, which accounts for almost all of the functional changes seen in the impaired monocyte. Indeed, we have confirmed that the impaired monocyte does have suppressed IKK and NFKB functionality. Our analysis has also revealed that the pattern of change could also be explained by AP-1, particularly the pattern of MCP-1 production, thus defects in MAPK p38/ERK/JNK signaling could suppress AP-1 activity and contribute to monocyte impairment. Furthermore, 
the most likely cause for decreased HLA-DR expression out with NFKB suppression is decreased JAK-STAT1 activation. 
Table 7. Top 5 networks involved in monocyte impairment based on pathway analysis (Metacore ${ }^{\mathrm{TM}}$ )

\begin{tabular}{|c|c|c|c|c|c|c|c|c|}
\hline No. & $\begin{array}{l}\text { Key } \\
\text { network } \\
\text { objects }\end{array}$ & GO Processes & $\begin{array}{l}\text { Total } \\
\text { nodes }\end{array}$ & $\begin{array}{l}\text { Seed } \\
\text { nodes }\end{array}$ & Pathways & p-Value & zScore & gScore \\
\hline 1 & NF-kB & $\begin{array}{l}\text { positive regulation of immune system process } \\
(100.0 \%) \text {, cellular response to lipopolysaccharide } \\
(75.0 \%) \text {, cellular response to molecule of bacterial } \\
\text { origin }(75.0 \%) \text {, positive regulation of leukocyte } \\
\text { migration }(66.7 \%) \text {, leukocyte chemotaxis }(66.7 \%)\end{array}$ & 12 & 11 & 0 & $7.78 \mathrm{e}-46$ & 323.72 & 323.72 \\
\hline 2 & AP-1 & $\begin{array}{l}\text { positive regulation of leukocyte migration }(72.7 \%) \text {, } \\
\text { leukocyte chemotaxis }(72.7 \%) \text {, leukocyte } \\
\text { migration }(81.8 \%) \text {, regulation of leukocyte } \\
\text { migration }(72.7 \%) \text {, positive regulation of } \\
\text { intracellular signal transduction }(100.0 \%)\end{array}$ & 11 & 10 & 0 & $1.98 \mathrm{e}-41$ & 307.38 & 307.38 \\
\hline 3 & $\begin{array}{l}\text { RelA (p65 } \\
\text { NF-kB } \\
\text { subunit) }\end{array}$ & $\begin{array}{l}\text { cellular response to lipopolysaccharide ( } 81.8 \%) \text {, } \\
\text { cellular response to molecule of bacterial origin } \\
(81.8 \%) \text {, positive regulation of leukocyte migration } \\
(72.7 \%) \text {, cellular response to biotic stimulus } \\
(81.8 \%) \text {, leukocyte chemotaxis }(72.7 \%)\end{array}$ & 11 & 10 & 0 & $1.98 e-41$ & 307.38 & 307.38 \\
\hline 4 & $\begin{array}{l}\text { NF-kB } \\
\text { p50/p65 }\end{array}$ & $\begin{array}{l}\text { cellular response to lipopolysaccharide ( } 81.8 \%) \text {, } \\
\text { cellular response to molecule of bacterial origin } \\
(81.8 \%) \text {, positive regulation of leukocyte migration } \\
(72.7 \%) \text {, cellular response to biotic stimulus } \\
(81.8 \%) \text {, leukocyte chemotaxis }(72.7 \%)\end{array}$ & 11 & 10 & 0 & $1.98 \mathrm{e}-41$ & 307.38 & 307.38 \\
\hline 5 & C-Jun & $\begin{array}{l}\text { positive regulation of ERK1 and ERK2 cascade } \\
(80.0 \%) \text {, positive regulation of leukocyte migration } \\
(70.0 \%) \text {, regulation of ERK1 and ERK2 cascade } \\
(80.0 \%) \text {, leukocyte chemotaxis }(70.0 \%) \text {, leukocyte } \\
\text { migration }(80.0 \%)\end{array}$ & 10 & 9 & 0 & $4.27 e-37$ & 290.14 & 290.14 \\
\hline
\end{tabular}




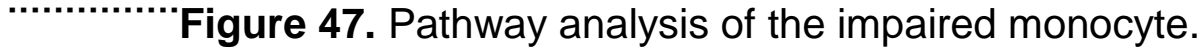

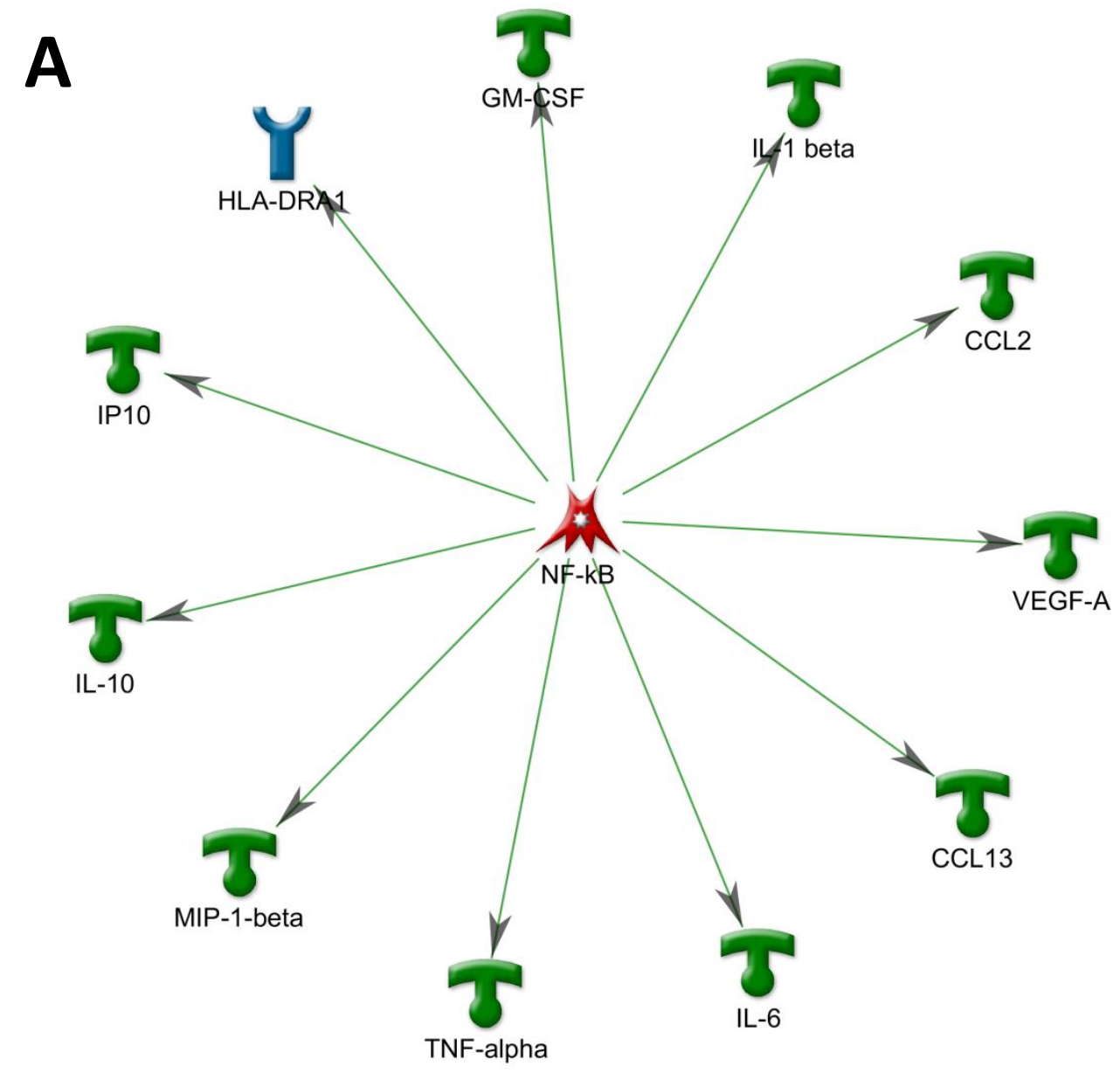

B

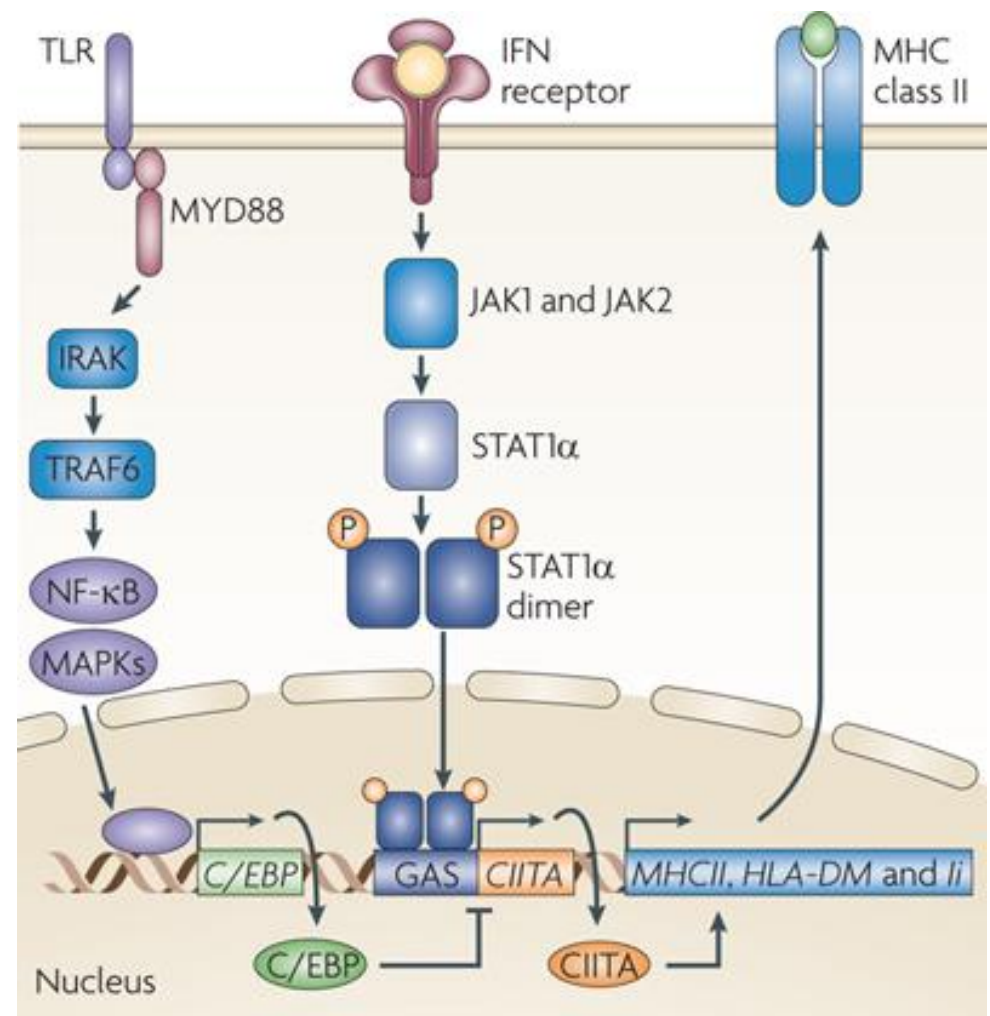




\section{Legend to Figure 47.}

Pathway analysis of the impaired monocyte. The most significantly affected signaling network, and indeed the network with the highest Z-score was NFKB This network, based on the differentially expressed cytokine/chemokine and surface protein levels in the impaired monocyte, is demonstrated in Figure A. Experimental evidence from the peer-reviewed literature and network analysis demonstrates a proven causal link between the function of NFKB and each downstream protein. The regulation of HLA-DR expression is primarily governed by the class II transactivator (CIITA). Figure $\boldsymbol{B}$ demonstrates the important role of the JAK-STAT pathway in promoting HLA-DR expression. Decreased HLA-DR in the impaired monocyte, which does not appear to be due to IKK/NFKB mediated suppression, but more likely decreases in the function of JAK-STAT pathway.

Source for A: MetaCore ${ }^{T M}$, Thomson-Reuters (from entering own data).

Source for B: Harding \& Boom, Nature Reviews Microbiology 8, 296-307 129 


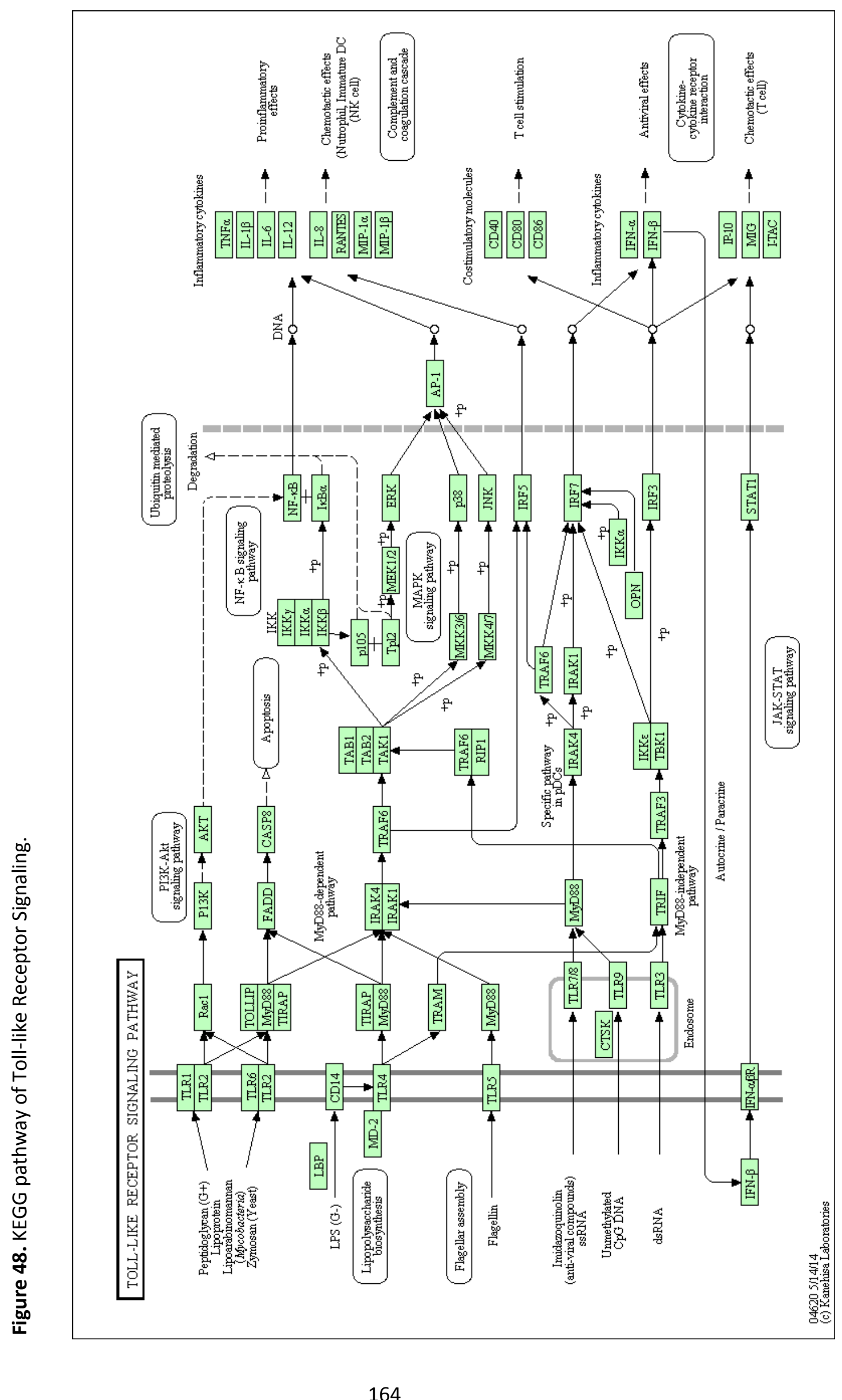




\section{CHAPTER VIII \\ ENHANCING IMPAIRED HOST DEFENCE MECHANISMS: AN \\ IMMUNOLOGICAL BASIS FOR ADJUVANT THERAPIES*2 \\ INTRODUCTION}

Surgical infection continues to plague the surgeon and the patient. Despite various strategies to address this problem, surgical site infection (SSI) still occurs in up to 17 per cent of patients, with post-operative infection of some kind affecting $23-49$ per cent of patients in high-risk groups. ${ }^{10}$ In particular, major elective surgery acts as a significant physiological insult to some of our patients.

Host defense impairment, and in particular, impairment of monocyte function, is generally accepted as being predictive of nosocomial or secondary infection after surgical trauma. ${ }^{4}$

Immunomodulatory agents have been studied clinically for many years, particularly in the context of trauma and sepsis without definitive outcomes. ${ }^{87}$ Some therapies appear to show promise by improving survival in murine models of sepsis, such as cecal ligation and puncture. However, these mice or rat models carry serious limitations: subjects are genetically homogenous and various clinical factors observed in humans are not taken into account. ${ }^{9}$ When these therapies are translated to humans, repeatedly the benefits in

\footnotetext{
${ }^{2}$ We plan to will be submit the work of this chapter to a peer-reviewed journal in this calendar year (2016).
} 
mortality are not consistently observed. In clinical practice, the majority of patients will have relatively normal monocyte function and do not stand to benefit from adjunctive therapy. A better understanding of how those with impaired monocyte function will respond could allow for better translation to the patient. The issue of monocyte impairment itself is hugely variable among patients and often unpredictable, but approximately one in six trauma patients have subnormal monocyte function. Thus, we have adopted an approach of studying human monocytes ex-vivo. Low-dose stimulation of cells by LPS (10 $\mathrm{ng} / \mathrm{mL}$ ) recreates not just the initial pro-inflammatory response observed clinically but also results in subsequent suppressed monocyte responsiveness to LPS exposure. The use of different healthy volunteers introduces variation from person to person, but permits the resulting impairment seen in some patients in a reproducible fashion. ${ }^{7,8}$

In this study, we have examined the underlying immunological basis for established and new adjuvant therapies using an ex-vivo model of impaired human monocytes to determine their influence on key host defence mechanisms. The immunomodulatory therapies we have focused on are interferon-y, GM-CSF and IKK-16 (an IKK inhibitor). 


\section{METHODS}

\section{Techniques for monocyte isolation and cytokine analysis are detailed in the main Methods section (page 61).}

\section{Flow cytometry}

Monocyte samples (100 $\mu \mathrm{L}, 100,000$ cells) were stained with fluorescein isothiocyanate-labeled anti-human CD14, phycoerythrin-labeled anti-HLA-DR antibodies (BD Biosciences, La Jolla, CA) and separately phycoerythrinlabeled anti-PD-L1 (BD Biosciences, La Jolla, CA) to measure monocyte surface expression of these proteins. Manufacturer instructions were closely followed. Monocytes were pelleted by centrifugation, washed with Dulbecco Phosphate Buffered Saline (Sigma Aldrich, St. Louis, MO), and fixed in 300 $\mu \mathrm{L}$ of 1 per cent paraformaldehyde solution.

At the indicated time points, monocyte surface levels of CD14, HLA-DR and PD-L1 expression were analysed using a FACS Calibur flow cytometer (Becton Dickinson, San Diego, CA). A total of 5,000 gated events were acquired. Mean fluorescence intensity (MFI) for each surface marker was analysed on monocytes using Cell Quest software (Becton Dickinson, San Diego, CA).

\section{Adjuvant therapies}

Human recombinant IFN-y, GM-CSF and IkK-16 were purchased from Sigma Aldrich, St. Louis (MO). Compounds were reconstituted from stock solution as per manufacturer's instructions and diluted in media to the appropriate concentrations.Adjuvant therapy doses were as follows: $100 \mathrm{ng} / \mathrm{mL} \mathrm{rIFN}-\mathrm{\gamma}$, 
$100 \mathrm{ng} / \mathrm{mL}$ rGM-CSF and $50 \mathrm{nM}$ IKK-16 as determined by dose-response experiments (data not shown). Adjuvants rIFN-y and rGM-CSF were then added to cell culture $1 \mathrm{~h}$ after isolation, simultaneously with $10 \mathrm{ng} / \mathrm{mL}$ LPS for a duration of $16 \mathrm{~h}$ (until $17 \mathrm{~h}$ post-isolation). IKK-16 was given at time zero for $1 \mathrm{~h}$, cells were washed with PBS and re-suspended in fresh media then 10 $\mathrm{ng} / \mathrm{mL}$ LPS was added to cell culture. No further doses of immunoadjuvants were given at or after the $100 \mathrm{ng} / \mathrm{mL}$ LPS challenge at $17 \mathrm{~h}$.

\section{Statistical Analysis}

Each donor was used as its own control. Wilcoxon-signed ranked test was used for calculation of significant differences where appropriate. Naïve conditions (no LPS 10ng/mL pre-treatment) and then each immunoadjuvant therapy were compared to the impaired monocyte. Significance was set at $P<$

0.05. Preliminary experiments were used to estimate that a sample size of 7 was required to obtain a power of $0.8(80 \%)$. SigmaPlot was used for presentation of data and for statistical analysis (SyStat Software, Inc., Chicago, IL). 
Figure 49. Experimental protocol for adjuvant therapies in the impaired monocyte.

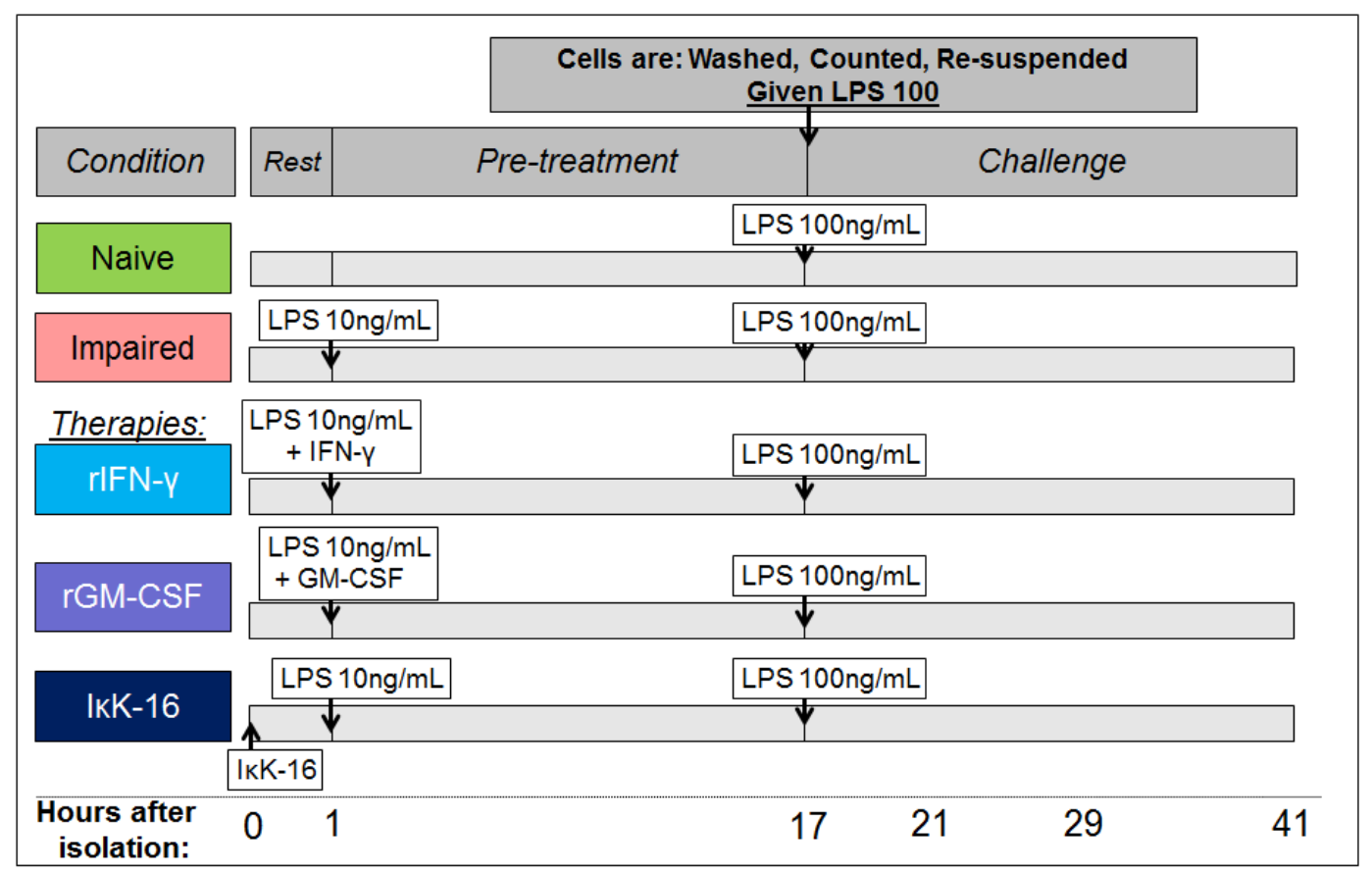

Timeline of the experimental protocol. Following monocyte isolation from healthy volunteers, following 1 hour of rest, cells are cultured either in media only (naïve) or with low dose $10 \mathrm{ng} / \mathrm{mL}$ LPS for 16 hours. Monocytes are then re-suspended in fresh media and challenged with high dose $100 \mathrm{ng} / \mathrm{mL} L P S$ to assess the inflammatory response. Adjuvant strategies aim to modulate the response to this $100 \mathrm{ng} / \mathrm{mL}$ LPS challenge. 


\section{RESULTS}

\section{Monocyte impairment}

Low-dose $10 \mathrm{ng} / \mathrm{mL}$ LPS stimulated ex-vivo human monocytes in culture to produce higher levels of TNF- $\alpha$ at $17 \mathrm{~h}$ compared with its naïve control (Figure 50). However, when these cells met a subsequent larger LPS challenge of $100 \mathrm{ng} / \mathrm{mL}$, they exhibited decreased levels of TNF- $\alpha$ when compared to the naïve cells, most noticeably at $21 \mathrm{~h}$. Monocytes impaired by low-dose LPS exposure also exhibited a decreased ability to produce IL-10, an anti-inflammatory cytokine, following the LPS challenge. This decrease in IL-10 levels was sustained, reaching statistical significance at 21, 29 and $41 \mathrm{~h}$ after the LPS challenge as seen in Figure 51. Monocyte surface HLA-DR expression, a parameter reflecting antigen-presenting capacity measured by flow cytometry, was modestly but consistently decreased in the LPS $10 \mathrm{ng} / \mathrm{mL}$ exposed monocyte at $17 \mathrm{~h}$ (Figure 52). CD14 and PD-L1 expression was similar between naïve and impaired conditions.

\section{IFN-y treatment}

Recombinant IFN-y treatment increased the capacity for the monocyte to produce TNF- $\alpha$. In response to the initial $10 \mathrm{ng} / \mathrm{mL}$ LPS, TNF- $\alpha$ levels increased in the impaired monocyte. Subsequent to $100 \mathrm{ng} / \mathrm{mL}$ LPS challenge at $17 \mathrm{~h}$, levels of TNF- $\alpha$ production were higher in IFN- $\mathrm{\gamma}$ treated monocytes at 21 and $29 \mathrm{~h}$. IL-10 levels appeared to initially decrease in IFN-y treated monocytes at $17 \mathrm{~h}$, but did not demonstrate significant differences. Compared 
to the impaired monocyte, HLA-DR expression was consistently sustained in response to IFN-y treatment at 21, 29 and $41 \mathrm{~h}$. Monocyte CD14 expression was decreased as a consequence of IFN- $\gamma$ treatment, most noticeably at $41 \mathrm{~h}$ (Figure 53). IFN-y treated monocytes had markedly increased PD-L1 expression at every time point (Figure 54).

\section{GM-CSF treatment}

GM-CSF appeared to decrease TNF- $\alpha$ and increase IL-10 at $17 \mathrm{~h}$, and then at all subsequent time points following $100 \mathrm{ng} / \mathrm{mL}$ LPS. However, these differences never reached statistical significance. GM-CSF did not have any significant influence on monocyte HLA-DR, CD14 or PD-L1 expression.

\section{IKK-16 treatment}

Despite the short exposure to IKK-16, there was a consistent and sustained decrease in TNF- $\alpha$ production when compared to the impaired monocyte, most noticeable at $17 \mathrm{~h}$ and $41 \mathrm{~h}$. Despite a decrease in TNF- $\alpha$, there was no decrease in IL-10 levels. In fact, monocytes treated with IKK-16 trended towards an increased capacity to produce IL-10 following an LPS challenge when compared with the impaired monocyte. IKK-16 also did not increase monocyte HLA-DR or PD-L1 expression; however, CD14 expression was progressively upregulated following the LPS challenge (Figure 53). 
Figure 50. Effect of immunoadjuvants on TNF- $\alpha$ production in the impaired monocyte.
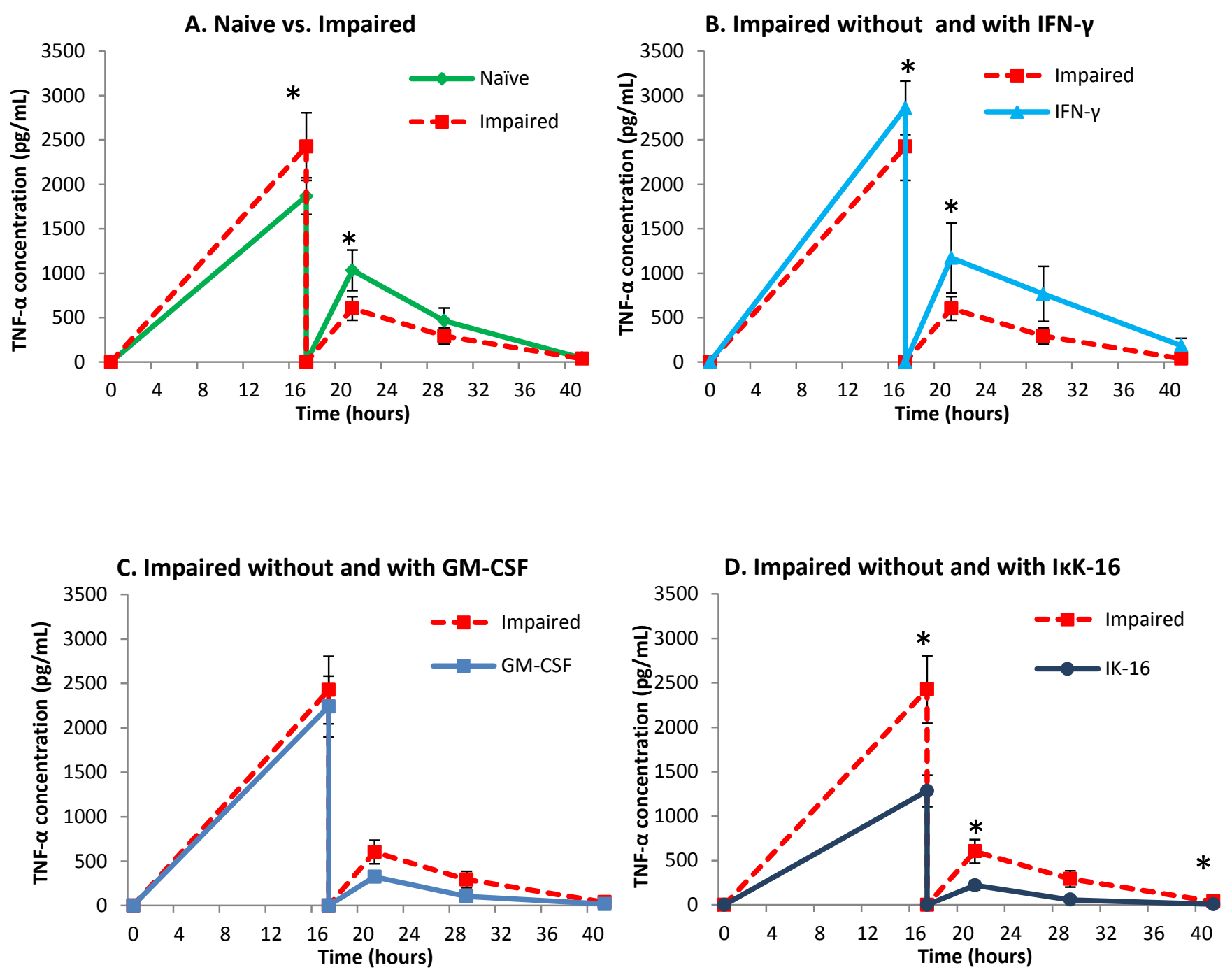

$\mathbf{N}=7$

Monocytes were cultured in naïve (media only) or impaired $(10 \mathrm{ng} / \mathrm{mL}$ )

conditions, with or without the three adjuvant regimes. Cells were then given the $100 \mathrm{ng} / \mathrm{mL}$ LPS challenge. Supernatant TNF-a protein was measured by ELISA. Mean \pm S.E.M. $N=7 .{ }^{*} p<0.050$. 
Figure 51. Effect of immunoadjuvants on IL-10 production in the impaired monocyte.
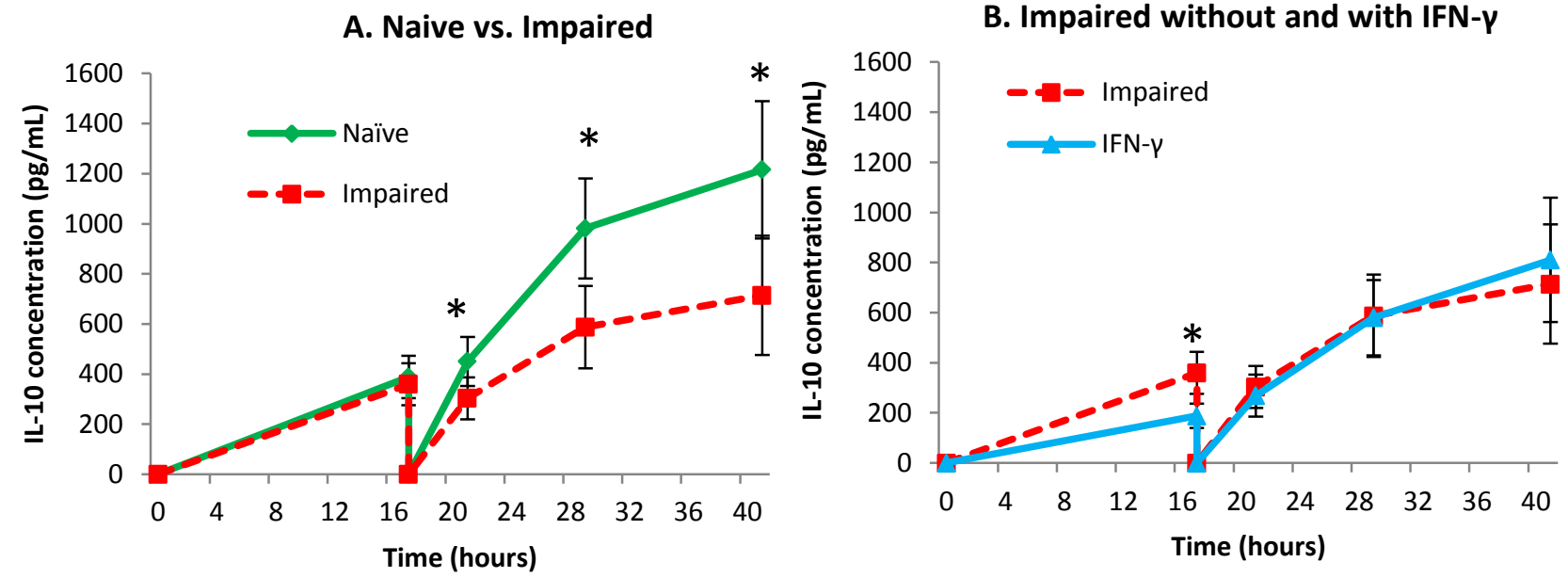

C. Impaired without and with GM-CS

D. Impaired without and with IKK-16
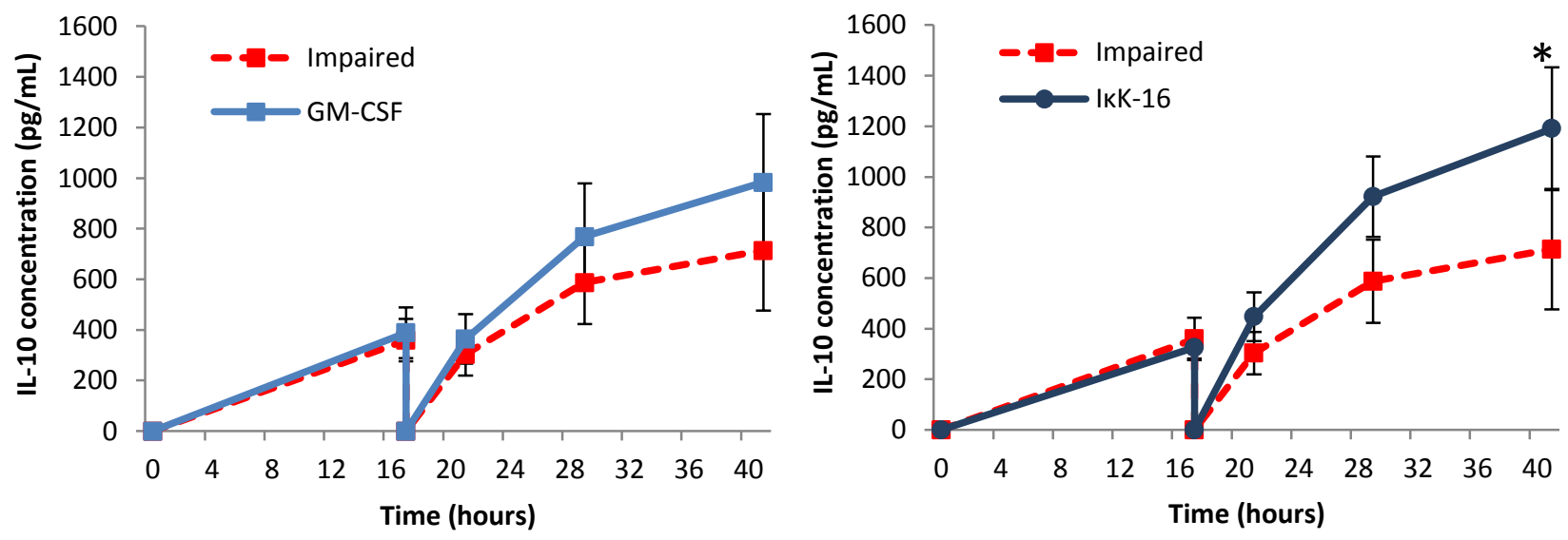

$\mathbf{N}=\mathbf{7}$

Monocytes were cultured in naïve (media only) or impaired (10ng/mL) conditions, with or without the three adjuvant regimes. Cells were then given the $100 \mathrm{ng} / \mathrm{mL}$ LPS challenge. Supernatant IL-10 protein was measured by ELISA. Mean \pm S.E.M. $N=7 .{ }^{*} p<0.050$. 
Figure 52. Effect of immunoadjuvants on HLA-DR expression in the impaired monocyte.

A. Naive vs. Impaired

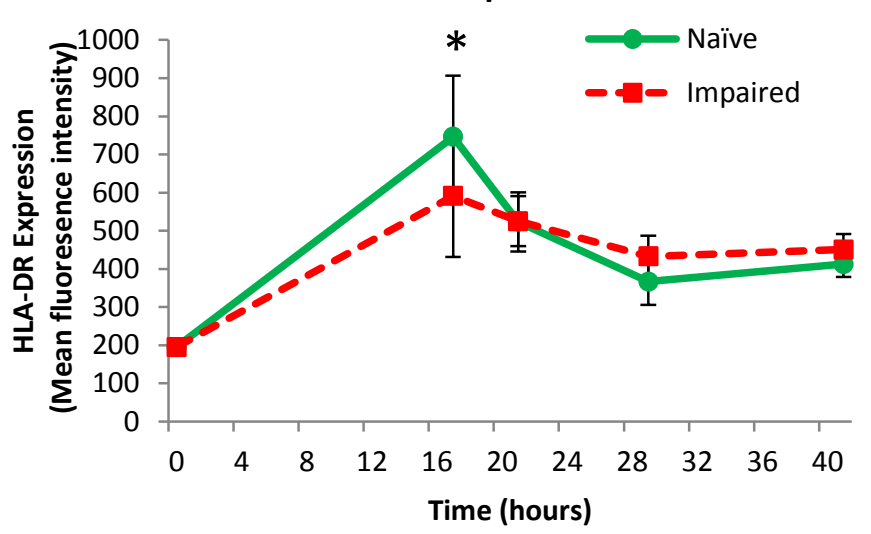

C. Impaired without and with GM-CSF

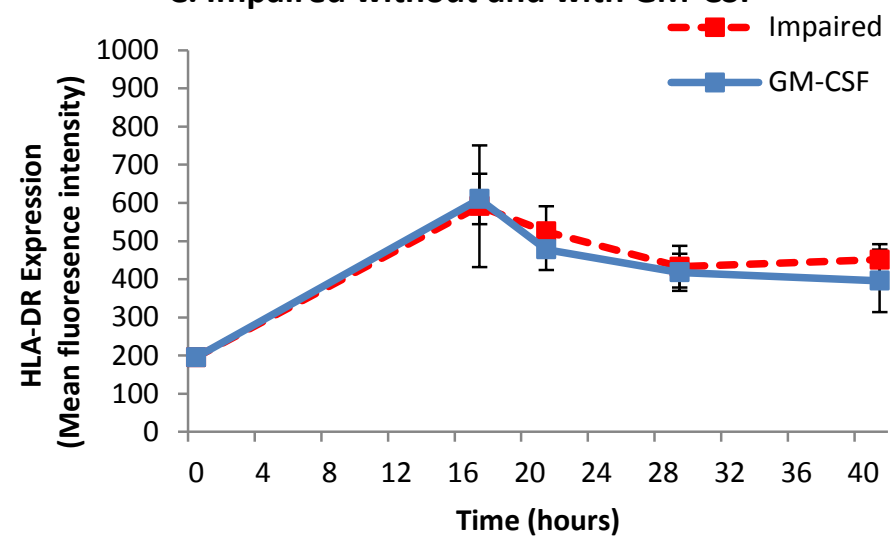

B. Impaired without and with IFN-p

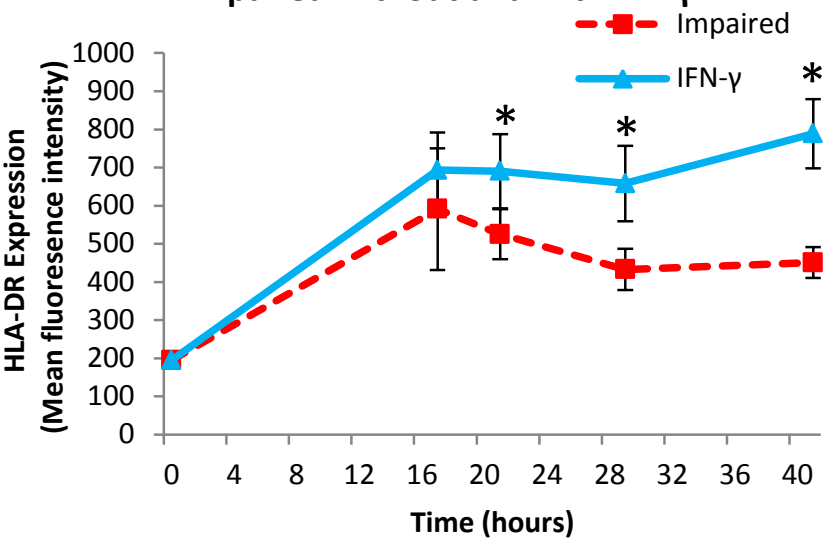

D. Impaired without and with IKK-16

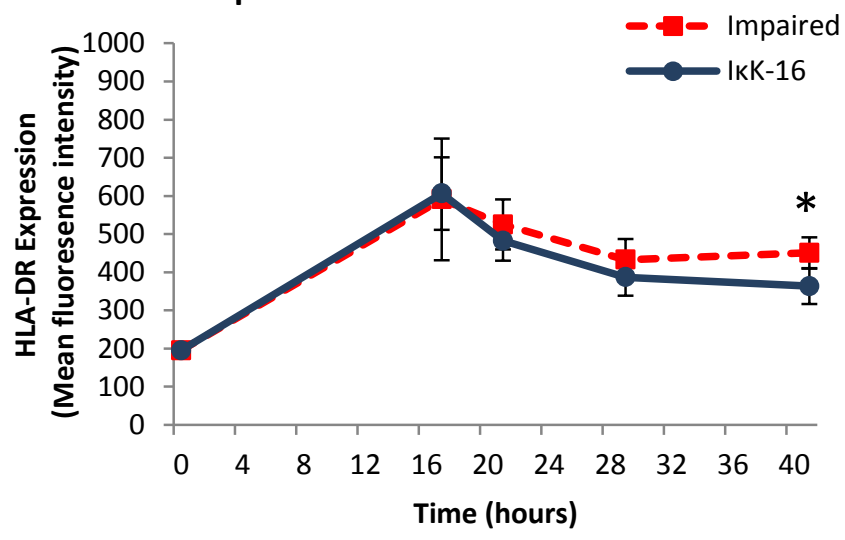

$\mathbf{N}=\mathbf{7}$

Monocytes were cultured in naïve (media only) or impaired (10ng/mL) conditions, with or without the three adjuvant regimes. Cells were then given the $100 \mathrm{ng} / \mathrm{mL}$ LPS challenge. Surface HLA-DR expression was measured by FACS. Mean \pm S.E.M. $N=7 .{ }^{*} p<0.050$. 
Figure 53. Effect of immunoadjuvants on surface CD14 expression in the impaired monocyte.
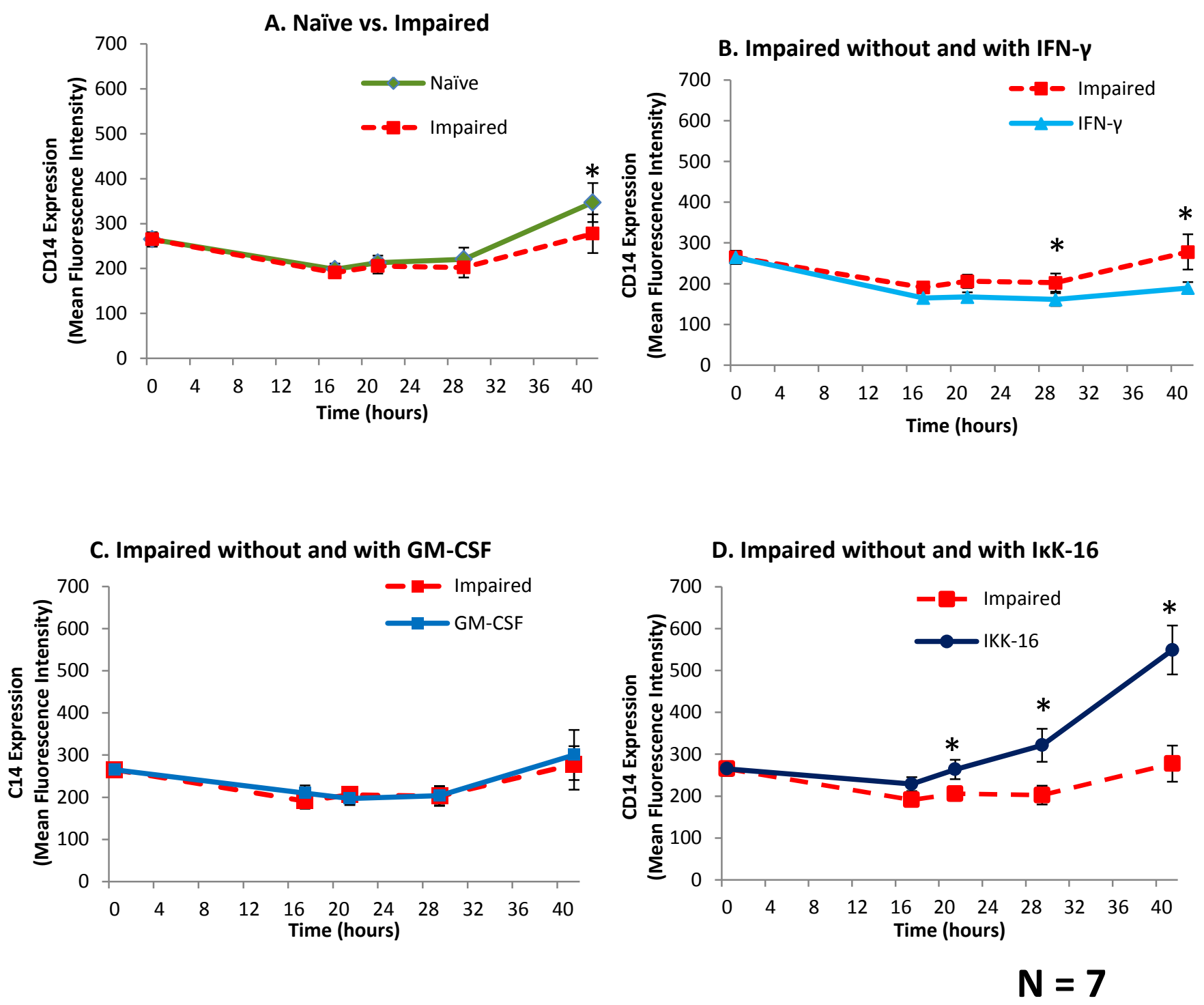

Monocytes were cultured in naïve (media only) or impaired (10ng/mL) conditions, with or without the three adjuvant regimes. Cells were then given the $100 \mathrm{ng} / \mathrm{mL}$ LPS challenge. Surface CD14 expression was measured by FACS. Mean \pm S.E.M. $N=7 .{ }^{*} p<0.050$. 
Figure 54. Effect of IFN- $\gamma$ on surface PD-L1 expression in the impaired monocyte.
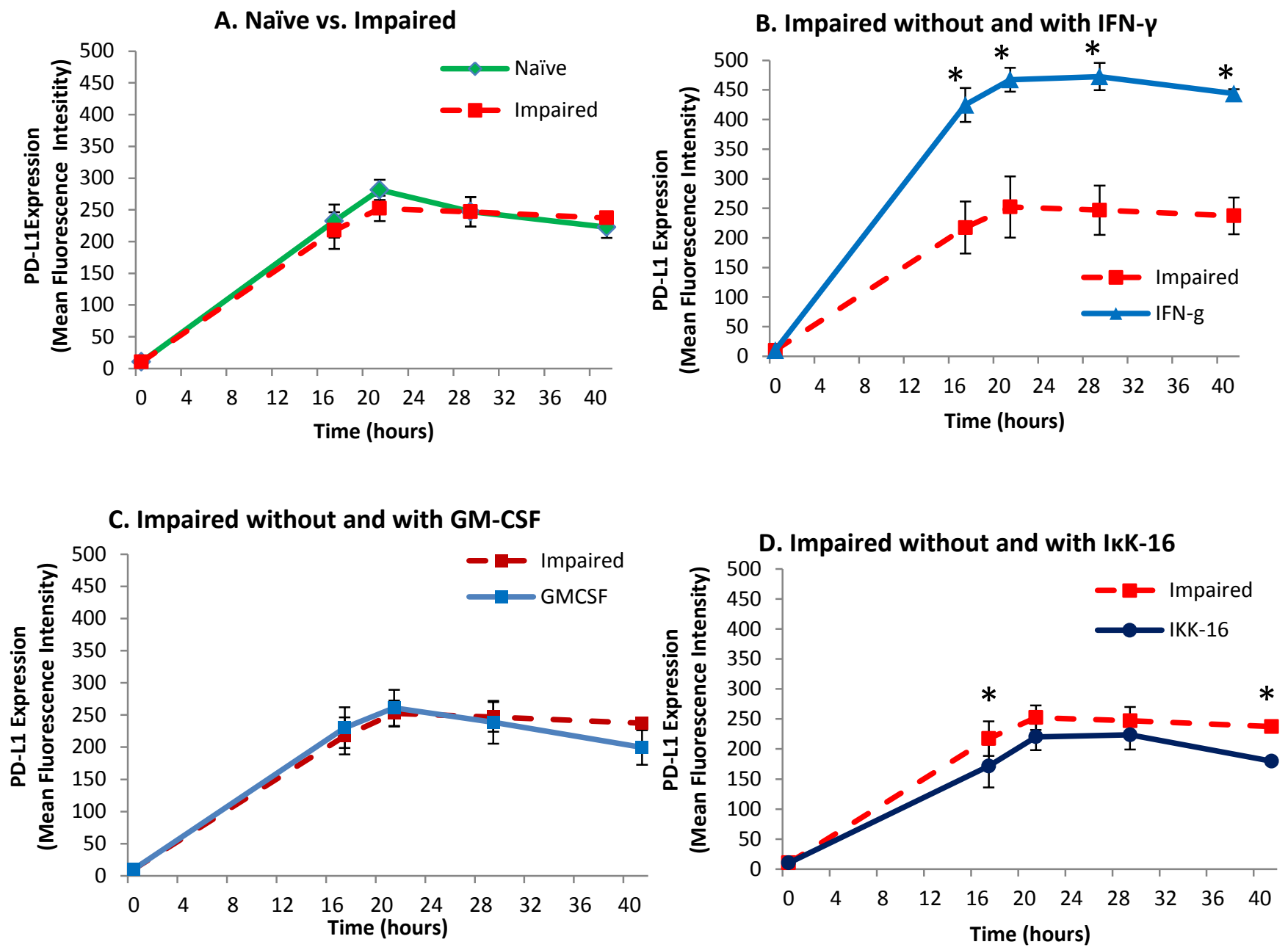

E. Effect of IFN- $\gamma$ (gray) on PD-L1

$\mathbf{N}=6$

Expression in the Impaired Monocyte

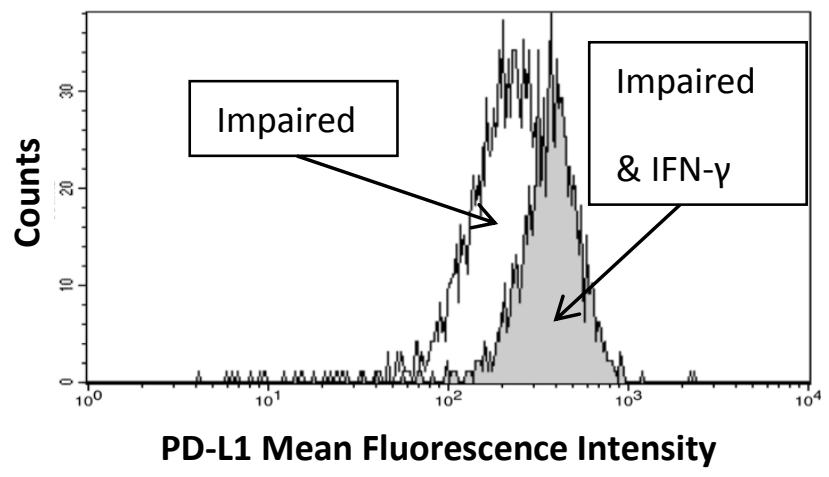




\section{Legend for Figure 54.}

Monocytes were cultured in naïve (media only) or impaired $(10 \mathrm{ng} / \mathrm{mL}$ ) conditions, with or without the three adjuvant regimens. Cells were then given the $100 \mathrm{ng} / \mathrm{mL}$ LPS challenge. Surface PD-L1 expression was measured by FACS. Mean \pm S.E.M. $N=7 .{ }^{*} p<0.050$. Figure $E$ demonstrates a representative histogram showing the increase in PD-L1 expression in response to IFN-y treatment. 
Figure 55. Effect of adjuvant treatments on monocyte viability.

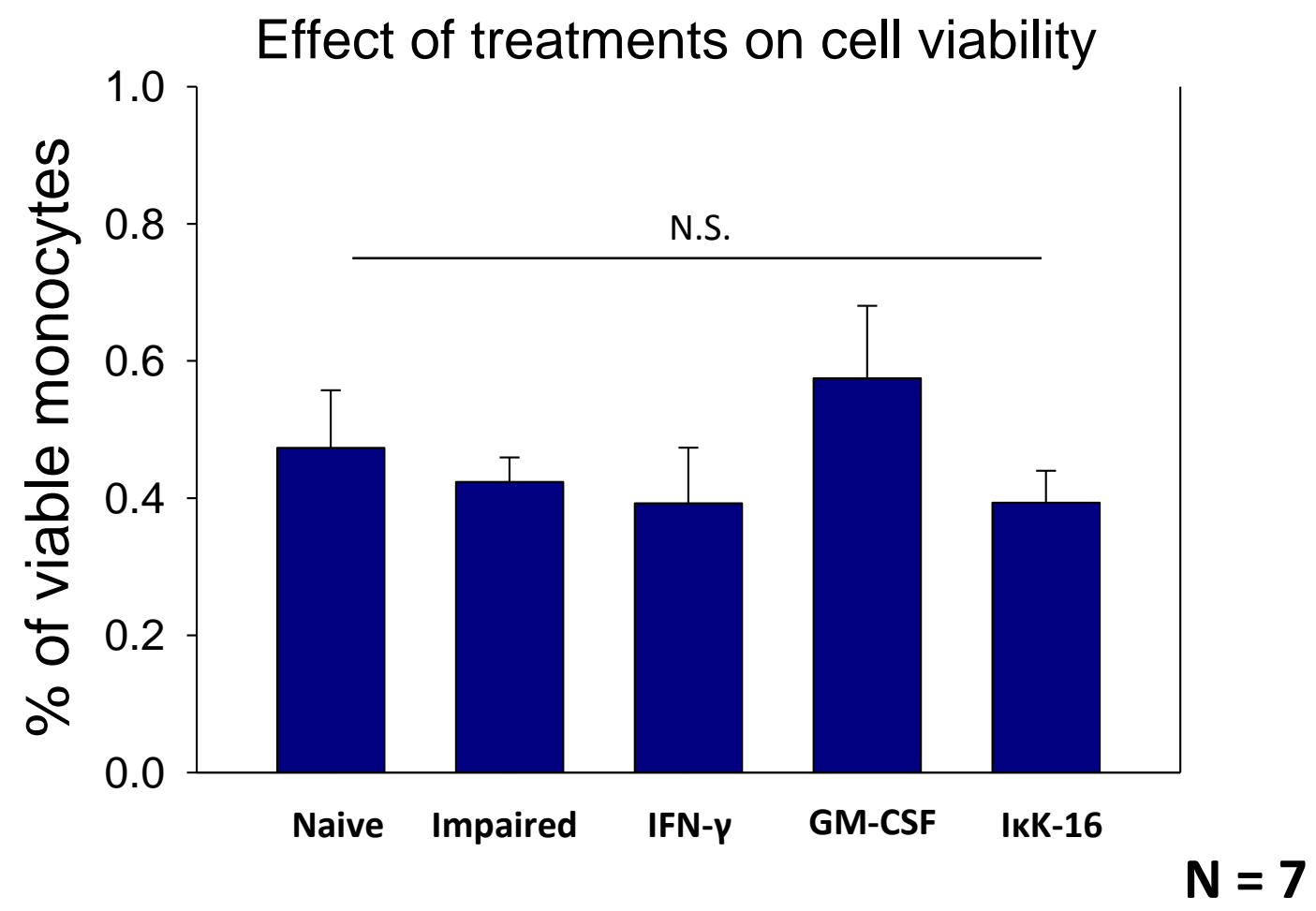

Monocytes were cultured on conditions of either naïve (media only), impaired (low dose $10 \mathrm{ng} / \mathrm{mL}$ LPS), or impaired with adjuvant as previously described for 16 hours, after an initial hour of rest. At 17 hours, cells were stained with Trypan blue and were counted by standard microscopy to determine cell viability between conditions. N.S., not statistically significant between groups (Repeated Measures ANOVA). 


\section{DISCUSSION}

The purpose of this study was to understand how potential immunoadjuvant therapies influence host defense mechanisms in a human ex-vivo model of monocyte impairment. Firstly, our data demonstrate that the initial response to endotoxin is a pro-inflammatory phenotype, but then impaired cytokine production occurs in response to a further endotoxin challenge. These findings are in agreement with many in-vitro and ex-vivo models in the literature. ${ }^{5,46}$ Furthermore, this model is representative of both human endotoxemia models and clinical studies examining the immune response after major elective surgery, trauma and sepsis. ${ }^{86}$ Specifically, the blunted capacity of monocytes to express HLA-DR and to produce TNF- $\alpha$ has repeatedly been shown to predict secondary infection and often death. This has formed the basis for numerous randomized clinical trials designed to enhance the monocyte inflammatory response. ${ }^{3,84}$

IFN- $\mathrm{y}$ has been shown to act through the IFN- $\mathrm{\gamma}$ receptor and subsequently via the JAK-STAT1 pathway to activate monocytes and macrophages. Our results show that IFN-y restores the capacity of the monocyte to produce TNF- $\alpha$ and augment HLA-DR expression. These findings are in keeping with previous in-vitro studies. ${ }^{47}$ In addition, these positive effects by IFN- $y$ have been shown in-vivo with numerous clinical studies. $^{91}$

We have shown that IFN- $y$ also increases the expression of PD-L1 on the surface of monocytes. Recent studies to understand the signaling and role of this negative co-stimulatory receptor have shown that IFN- $y$, through its 
activation of the JAK-STAT1 pathway, also leads to higher PD-L1 expression in natural killer cells. ${ }^{130}$ We believe this likely represents a negative feedback mechanism to suppress endogenous IFN-y by T-cells in the context of highly abundant exogenous sources. Future research is required to determine if PDL1 will be as clinically applicable as a marker or therapeutic target in the field of surgical infection as it has been in oncology.

Our data has not demonstrated any significant modulatory effect of GM-CSF on the impaired monocyte. Preliminary dose-determining studies suggested that IL-10 production was augmented in the naïve monocyte; however, this effect may be attenuated in the setting of monocyte impairment. Surgical trials using GM-CSF have shown increased peri-operative HLA-DR expression, and our results suggest that this effect may be through promotion of myelopoeisis to produce new, functioning monocytes rather than modulating existing circulating impaired monocytes. ${ }^{93}$

IKK-16 is a small molecule inhibitor of IKK, acting to suppress this proinflammatory signaling pathway. "Glue Grant" studies of the genomic response in surgical patients following trauma demonstrated that the magnitude of both the pro- and anti-inflammatory response determined patient outcome. ${ }^{1}$ Due to the presumed risks of persistent suppression of inflammation, a short exposure of this therapy was used. Our results show that, despite short exposure, decreases in TNF- $\alpha$ production are sustained. Interestingly, levels of the anti-inflammatory cytokine IL-10 were restored towards the level of the naïve monocyte. While the use of IkK-16 have improved end organ function in pre-clinical models of hemorrhagic shock and sepsis, tipping the balance in favor of IL-10 and decreasing TNF- $\alpha$ may in fact 
increase the risk of nosocomial infection in some patients. ${ }^{77}$ One unexpected finding of the effect of IKK-16 was the augmentation of CD14 expression, especially in the later stages after stimulation. CD14 acts as a receptor for LPS, binding to TLR4 homodimers for signal transduction in the monocyte. Studies in trauma patients have shown that the detachment of this receptor following excessive stimulation, examined either by decreased monocyte expression or increased soluble CD14, is a predictor of infection and death. ${ }^{131,132}$ We believe that by decreasing the early and late responses to LPS by limiting IKK activation, this might represent a mechanism to preserve the capacity to respond to future exposure to pathogens and may warrant further study.

This study does have some limitations. Due to the ex-vivo and artefactual nature of this study, we cannot account for how other immune cells might respond to these immunomodulatory agents. Furthermore, the leukocyte response to LPS shares $88 \%$ of the same genomic response to trauma, as demonstrated in high throughput Glue Grant studies. ${ }^{1}$

In conclusion, IFN-y is the most effective immunoadjuvant in terms of increased TNF- $\alpha$ production and HLA-DR expression, which are two important predictors of surgical infection. However, by modulating a different signaling pathway, IKK-16 restored IL-10 production and augmented CD14 expression. Correcting impaired host defenses is a key factor in preventing and surviving surgical infection. We, as surgeons, are still trying to modulate pathologic immune responses to improve rates of surgical infection and death in our patients. Careful clinical judgment in terms of patient selection and timing is required in successfully translating these studies to future clinical trials. 
CHAPTER IX

MONOCYTE FUNCTION IN THE INFECTED SURGICAL PATIENT

INTRODUCTION

Surgical patients are at risk from numerous different forms of nosocomial infection during their hospital course. They may present with an acute intra-abdominal infection such as diverticulitis, or from a contaminated wound following trauma. The critically unwell patient often has invasive lines which can act as a foreign body, and can be ventilated which carries a risk of pneumonia. Finally, surgical site infection (SSI) is of political importance as a potential measure of quality of care, and can range from superficial wound infection to deep organ or cavity space infection. Patients with SSI's often require additional interventions, have a longer hospital stay and experience greater morbidity. Previously in this dissertation, impaired host defense was highlighted as a risk factor in the probability of surgical site infection, often measured by low monocyte HLA-DR or low ex-vivo TNF-a production in response to a stimulus. Few studies have examined the immune response in surgical patients after the so called clinical "second hit" of infection.

In this chapter, surgical patients from a University Colon and Rectal Surgery practice who were diagnosed with infection were studied. Patients were selected based on being "in homeostasis with or tolerant of" their infective source i.e. likely to bear the clinical phenotype of in-vivo monocyte impairment previously described. This is distinct from other studies in sepsis 
where patients had a systemic inflammatory response with or without shock and multi-organ failure.

The aims of this study was 1) to determine monocyte function in major surgical patients with infection, as judged by HLA-DR expression and ex-vivo cytokine production and 2) investigate the expression of intracellular negative regulator molecules that could represent a potential marker or indeed driver of suppressed monocyte function.

\section{METHODS}

Following IRB approval and written informed consent, peripheral blood was obtained by phlebotomy. Blood samples were taken both during episodes of infection and after clinical recovery (median 60 days after $1^{\text {st }}$ sample). As a comparator group, peripheral blood was also obtained from healthy volunteers.

The majority of patients were hemodynamically stable and most were diagnosed with deep surgical site infection, as defined by an abscess in an organ or cavity space. The specific sources of infection included intraabdominal abscess $(n=12)$, pneumonia $(n=2)$, perineal abscess $(n=1)$ and line sepsis $(n=1)$. Patients were aged 25-81 years, 6:10 (M:F). Healthy volunteers were aged 19-32 years, 7:3 (M:F).

Peripheral blood was obtained using EDTA tubes, which prevented clotting and allowed ex-vivo testing of the immune response. Monocyte HLADR expression (FACS) and cytokine levels (ELISA) were examined at baseline $0 \mathrm{~h}$ (unstimulated) and following ex-vivo lipopolysaccharide (LPS) 
stimulation (100 ng/mL) for $4 \mathrm{~h}$ (Fig. 56). Plasma samples were obtained after centrifugation of whole blood, and stored at $-80^{\circ} \mathrm{C}$ until later testing. Standard individual ELISA assays were performed for TNF- $\alpha$, IL-10 and IL-6 (as described the main Methods section, from page 61). Flow cytometry was performed on whole blood using the protocol described in the main Methods section, from page 61 . The main difference in protocol was that whole blood was lysed with hypotonic lysis buffer to exclude RBCs from analysis. For the gating strategy, the monocyte population was selected based on forward and side scatter properties, and confirmed by gating only on CD14 positive cells . Monocyte HLA-DR is expressed as mean fluorescence intensity (MFI). The same antibodies for CD14 (FITC) and HLA-DR (PE) were used as described in the main Methods section (from page 61), however, samples were run using a BD LSRII flow cytometer (BD Biosciences, CA).

The remaining monocytes were isolated by magnetic bead positive selection and mRNA was extracted and analyzed via qRT-PCR, to compare the ratio of normalized mRNA expression (using 18S) between the patient and healthy control samples (as described in the main Methods section, from page 61). For statistical analysis, Mann-Whitney-U tests and Unpaired T-tests were used where appropriate. 
Figure 56. Experimental design of clinical study of patients with surgical infection.

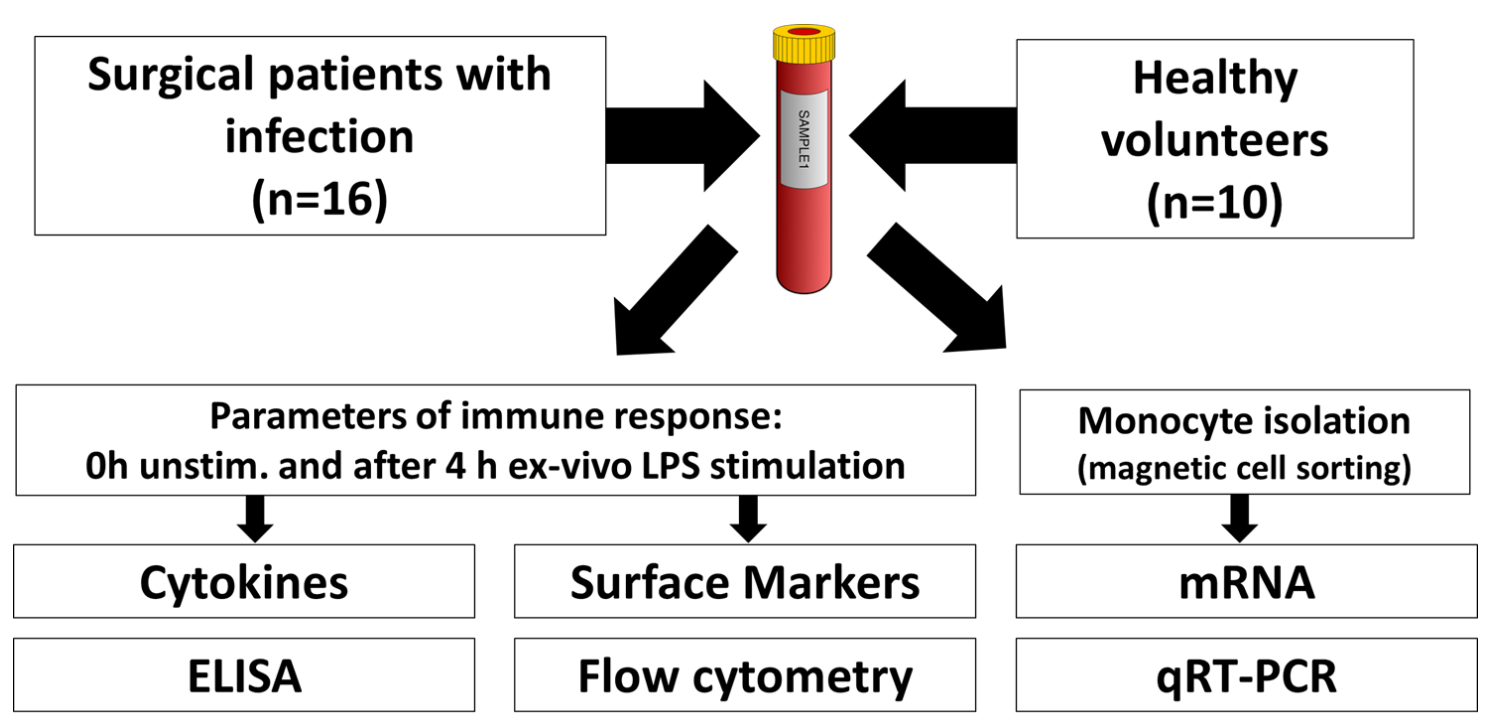

Peripheral blood was taken from surgical patients with infection. Monocyte function was determined by CD14 positive monocyte HLA-DR expression by FACS, and ex-vivo LPS stimulation in whole blood for 4 hours. At 4 hours, $H L A-D R$ expression was measured again to determine the response, as well as concentrations of plasma cytokines TNF- $\alpha, I L-10$ and IL-6 by ELISA. In addition, monocytes were isolated from patient samples using CD14 magnetic bead isolation, and monocyte RNA was extracted and qRT-PCR was used to determine the expression of various known negative regulators of monocyte function. Further samples were taken from patients later in their course of illness, presumably moving toward health after hospital discharge; healthy volunteers were also studied. 


\section{RESULTS}

\section{Monocyte HLA-DR expression}

Patients had statistically significant lower baseline HLA-DR expression (0h, unstimulated) compared to healthy volunteers (Figure 57). Following LPS stimulation, healthy volunteers upregulated HLA-DR expression to MFI of approximately 15,000 compared with only 8,000 in patients during their initial sample $(p<0.05)$. Levels of HLA-DR either prior to or after LPS stimulation did not seem to improve in follow-up samples compared with initial patient samples.

\section{Cytokine production}

Circulating levels of TNF- $\alpha$ were virtually undetectable in healthy volunteers and patients (Figure 58). The levels of TNF- $\alpha$ appear slightly higher in patients, however, these were below the lower limits of detection in the assay and did not reach statistical significance. Decreased monocyte TNF- $\alpha$ production was observed in patients in response to LPS stimulation, compared with healthy volunteers. Follow-up samples from patients also had lower levels of TNF-a production compared with health volunteers but also appeared lower than initial patient samples.

Circulating levels of IL-10 were increased in patients compared to the healthy controls (Figure 59, $\mathrm{p}<0.05$ ). Levels of IL-10 did decrease in the follow-up patient samples. After 4 hours of LPS stimulation, there were minimal increases in all groups in IL-10 production. IL-10 levels after 4 hours 
of LPS stimulation remained higher in patients compared with healthy volunteers.

Circulating plasma IL-6 levels (prior to stimulation) were elevated in the initial patient samples compared to healthy volunteers, in which IL-6 levels were below the lower limits of detection (Figure 60). Following 4 hours of exvivo LPS stimulation, this pattern was reversed. Healthy volunteers increased their IL-6 levels to approximately $2,000 \mathrm{pg} / \mathrm{mL}$, compared with a mean IL-6 level of less than $1,000 \mathrm{pg} / \mathrm{mL}$ in patients with infection. Follow-up samples for IL-6 were not available for analysis at the time of writing.

\section{Gene expression of negative regulators of the inflammatory response}

Based on a thorough literature search, the expression of the following negative regulator molecules were analysed; ATF3, IRAK-M, TNFAIP3 (A20), SOCS-1, SOCS-3 and SHP-1. These were compared to $18 \mathrm{~S}$ as the housekeeping gene for normalization. Gene expression of IRAK-M, a known negative regulator of inflammation, was consistently upregulated in patients with infection as compared to healthy controls $(p<0.05)$ (Figure 61). Data for the other negative regulators were not significantly different (data not shown). Interestingly, IRAK-M mRNA expression appeared to decrease after clinical recovery (dark gray) although this did not reach statistical significance. 
Figure 57. Monocyte HLA-DR expression in patients with surgical infection.
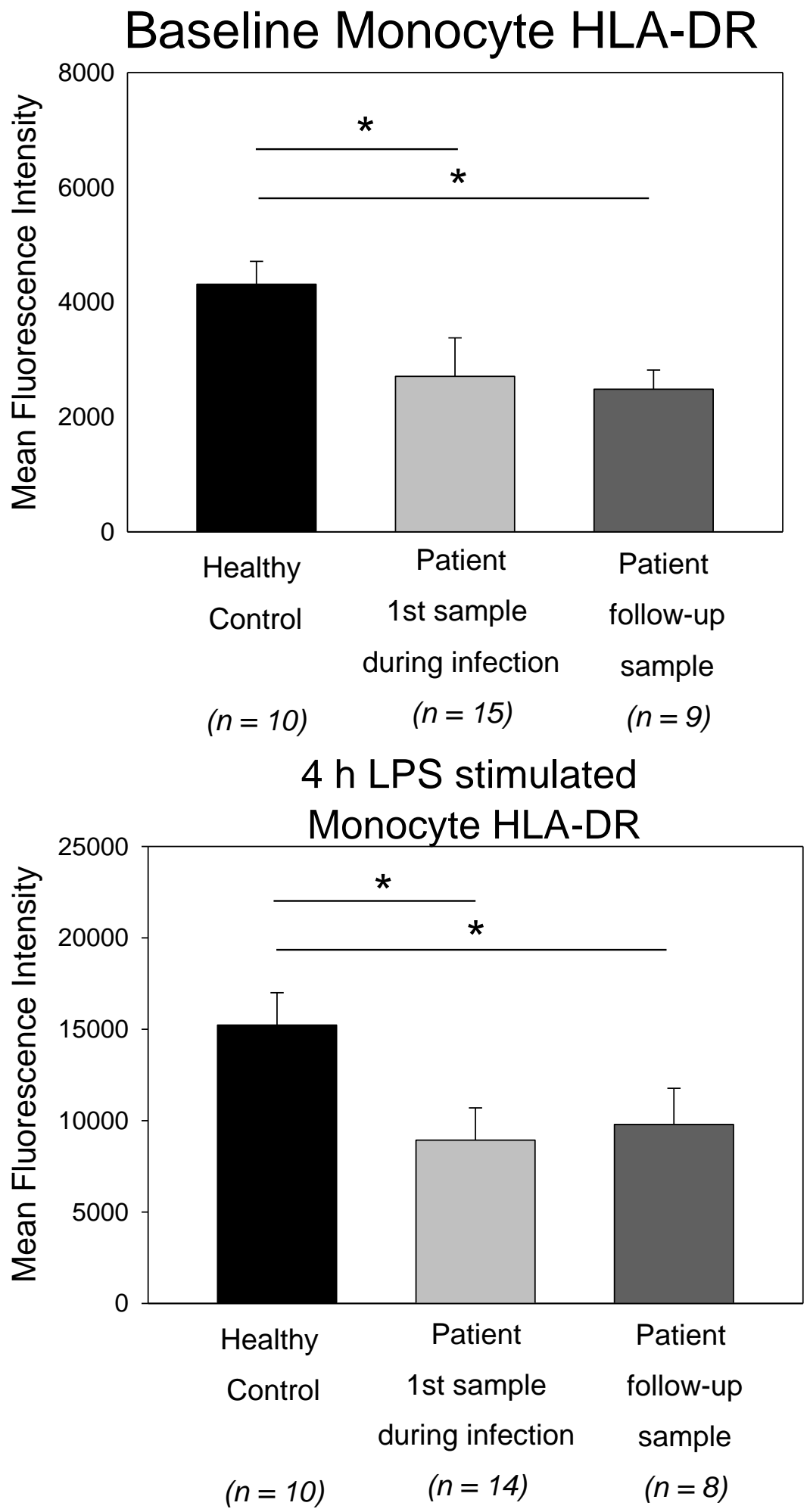

Monocyte HLA-DR expression was determined in both unstimulated blood (baseline $0 \mathrm{~h}$ ) and after $100 \mathrm{ng} / \mathrm{mL}$ LPS stimulation ex-vivo for 4 hours. HLADR expression using PE-tagged antibodies was determined on CD14 positive monocytes using FACS. * $p<0.05$, Mann-Whitney U test. 
Figure 58. Plasma TNF- $\alpha$ concentration in patients with surgical infection.

Baseline Plasma TNF- $\alpha$

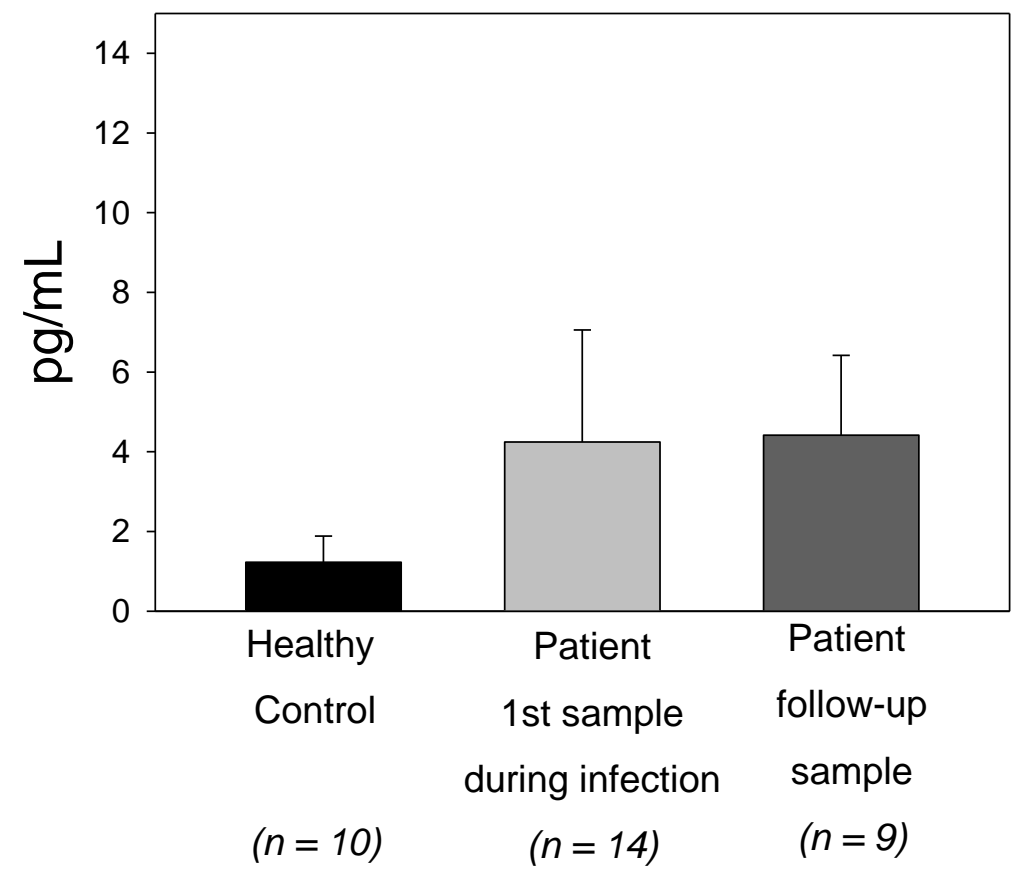

$4 \mathrm{~h}$ LPS stimulated

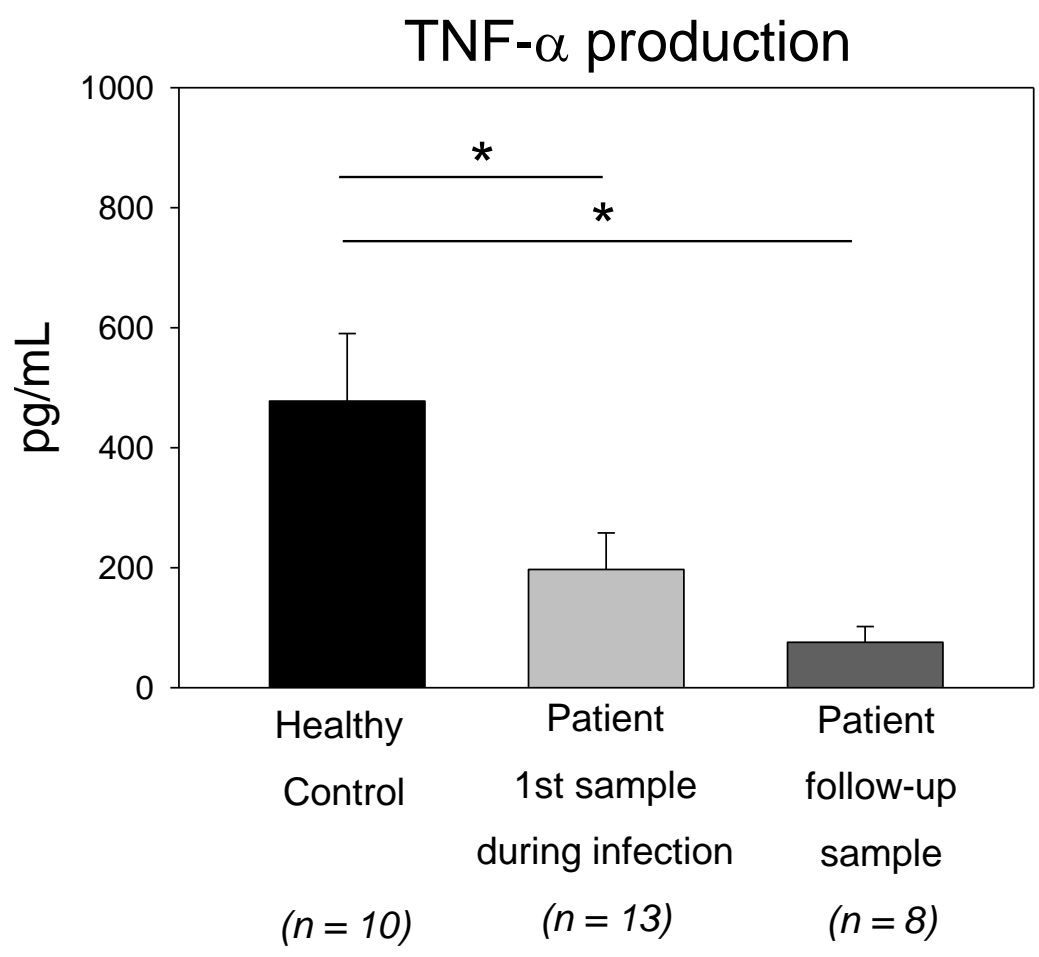

TNF- $\alpha$ concentration was determined in both unstimulated blood (baseline $0 \mathrm{~h}$ and after $100 \mathrm{ng} / \mathrm{mL}$ LPS stimulation ex-vivo for 4 hours by ELISA. ${ }^{*} p<0.05$, Mann-Whitney U test. 
Figure 59. Plasma IL-10 concentration in patients with surgical infection.
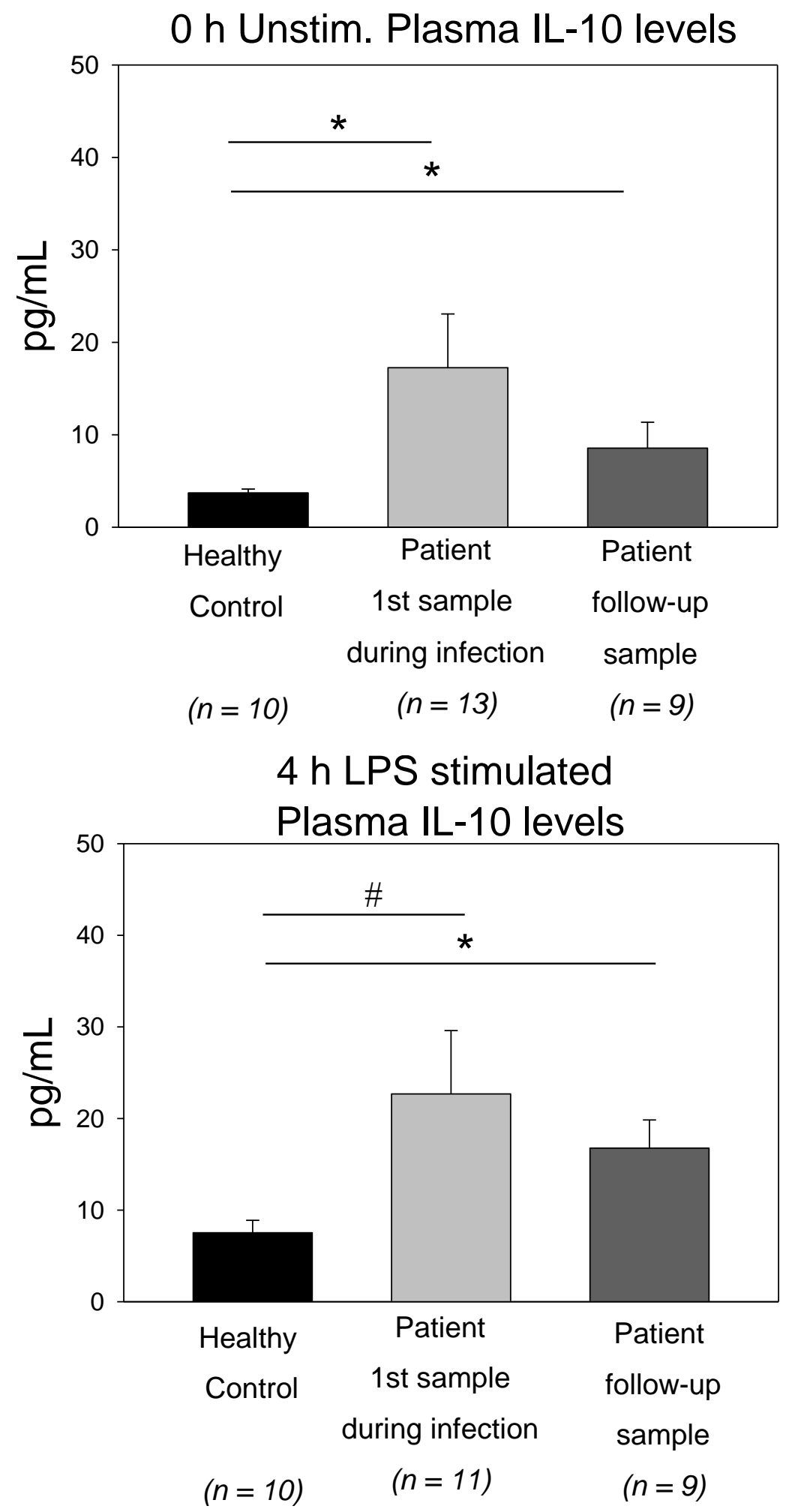

IL-10 concentration was determined in both unstimulated blood (baseline, $0 \mathrm{~h}$ ) and after $100 \mathrm{ng} / \mathrm{mL}$ LPS stimulation ex-vivo for 4 hours by ELISA. ${ }^{*} p<0.05$, Mann-Whitney U test. \# $p=0.057$. 
Figure 60. Plasma IL-6 concentration in patients with surgical infection.
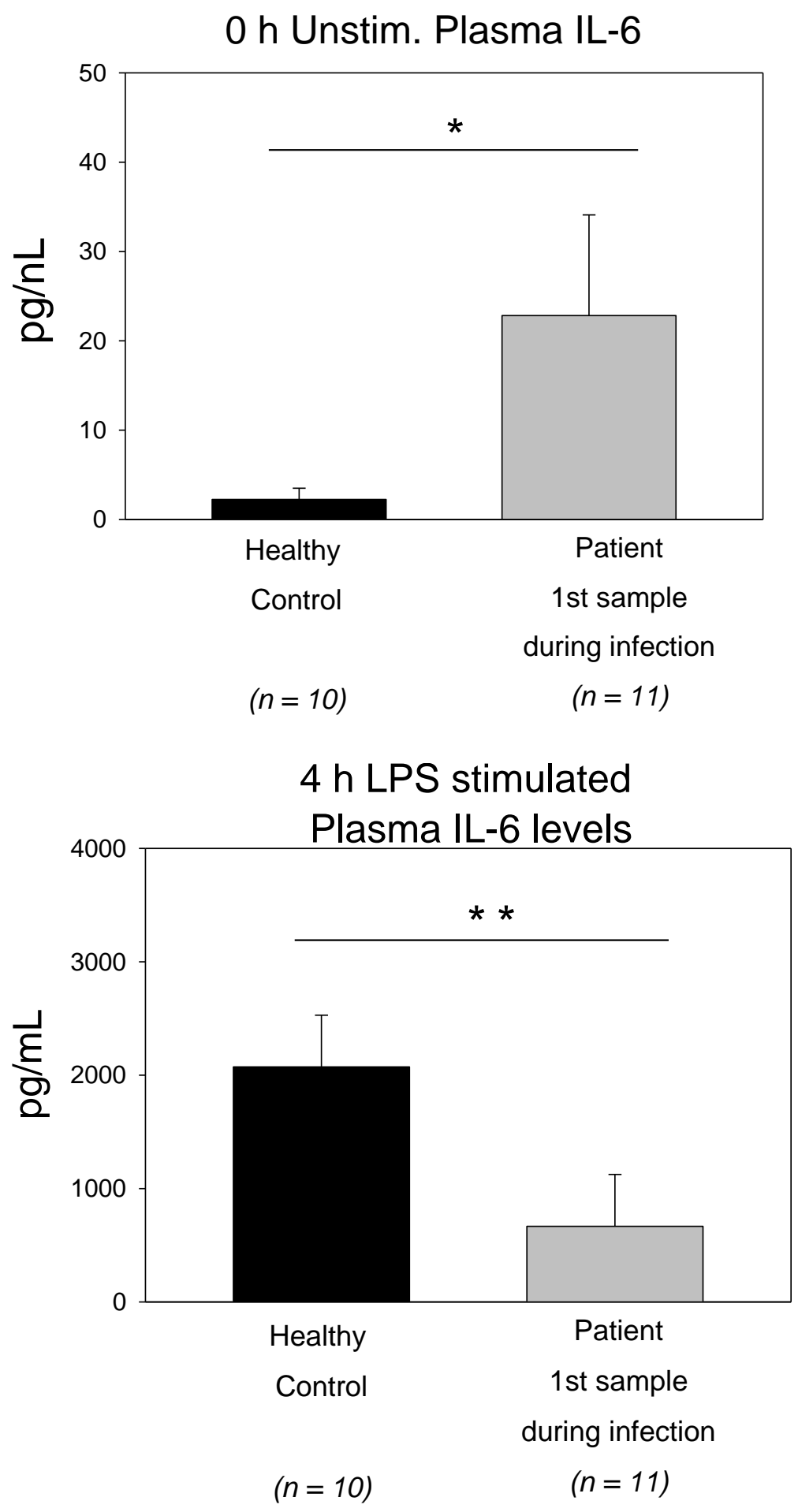

IL-6 concentration was determined in both unstimulated blood (baseline, $0 \mathrm{~h}$ ) and after $100 \mathrm{ng} / \mathrm{mL}$ LPS stimulation ex-vivo for 4 hours by ELISA. * $p<0.05$, Mann-Whitney U test. 
Figure 61. Monocyte IRAK-M expression in patients with surgical infection.

Monocyte IRAK-M

mRNA expression

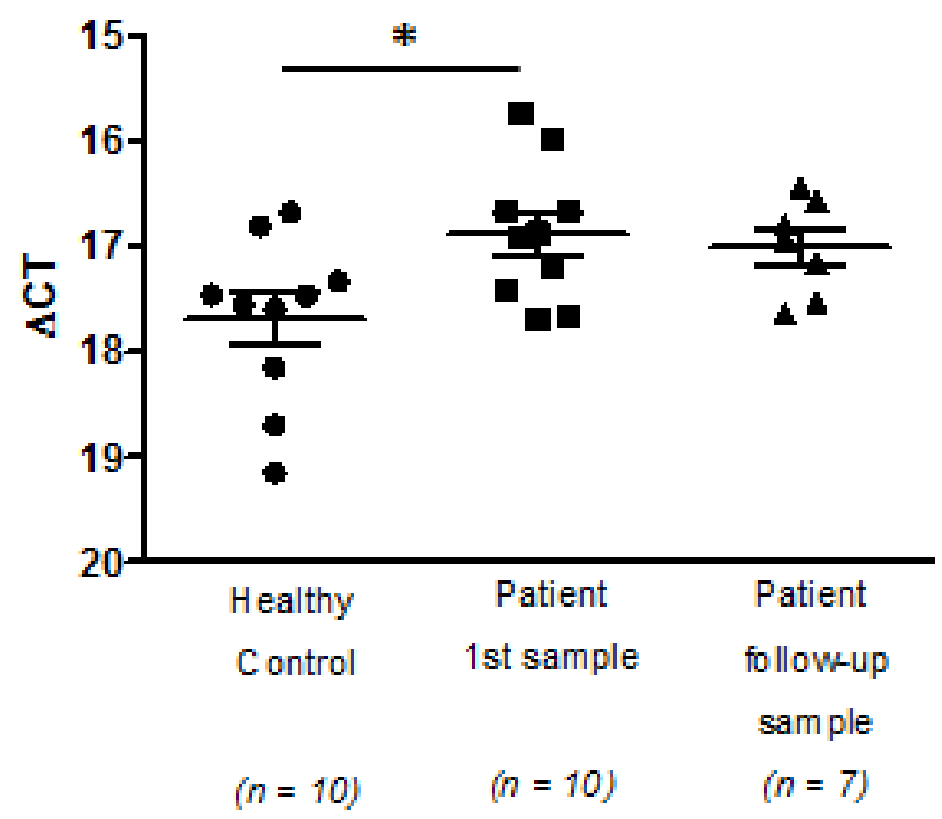

Monocyte IRAK-M mRNA expression

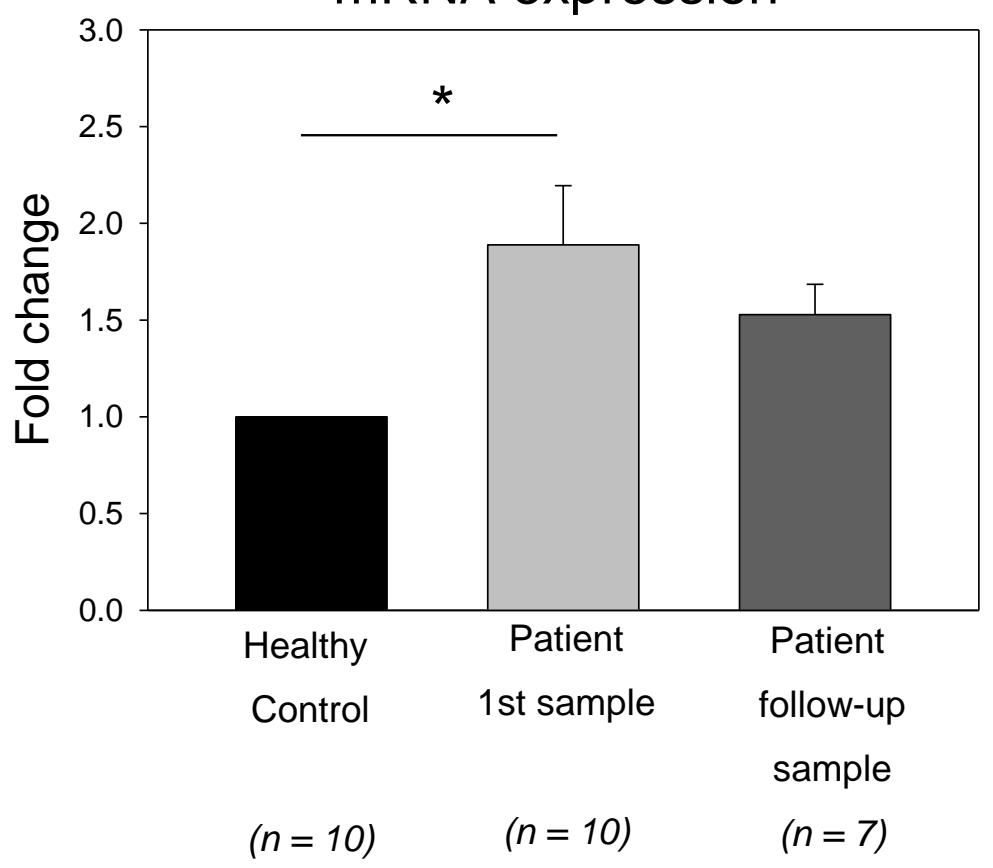

Monocyte IRAK-M expression was determined by qRT-PCR with data normalized to $18 \mathrm{~S}$. For fold changes, patient samples were compared to healthy controls. ${ }^{*} p<0.05$, Unpaired T-test. 


\section{DISCUSSION}

A proportion of surgical patients have a suppressed inflammatory response which impairs their ability to clear infection. This can result in the permissive escape of microbes from the chest, urine or wounds into the bloodstream, leading to bacteremia. As a result, multi-organ failure and death is a consequence in some patients. Prolonged or repeated physiological insults through repeated operations, infection or trauma, and certain therapies can lead to suppressed monocyte function. Such patients with "deactivated" host defenses or "immunoparalysis" can tolerate infective sources, such as abscesses, to persist without clearance. It was this clinical phenotype of surgical patient that was selected for study in this chapter.

Firstly, we aimed to determine monocyte function within these patients. Identification of such patients at higher risk for failure to clear infection is of clinical interest. Furthermore, such a patient may benefit from a therapy that could "reactivate" monocyte function. Much of the principle underpinning the isolated monocyte model of impairment design in Specific Aim 1 held the assumption that these patients had decreased ability to produce TNF- $\alpha$ in response to LPS as well as decreased levels of HLA-DR expression. These results confirm that the patients studied with surgical infection did have suppressed monocyte function, compared to healthy volunteers. Not only was monocyte HLA-DR expression decreased prior to stimulation, but the patient's monocytes were unable to upregulate HLA-DR expression in response to LPS stimulation. These findings are in keeping with prior studies in sepsis and trauma patients ${ }^{4,24}$. TNF- $\alpha$ levels were decreased in response to LPS, but 
also the capacity to produce IL-6 was decreased. These data confirm the validity of the impaired monocyte model.

Interestingly, circulating plasma levels of IL-6 and also IL-10 prior to stimulation were elevated in patients compared with healthy controls. This is in keeping with results from other groups ${ }^{27,133}$. Mechanistically, this could in part explain the decreased monocyte responsiveness. IL-10 is known to decrease TNF- $\alpha$ gene expression and downregulate HLA-DR expression. IL10 has previously been discussed as a potential target to block and prevent this loop of monocyte impairment ${ }^{134}$.

A difference between the impaired monocyte model and the patient observations is the increased IL-10 levels observed in infected patients. In our model, the impaired monocyte consistently suppresses IL-10 production. There are various explanations for this. Firstly, these samples were taken from whole blood rather than a single cell suspension of monocytes. Other documented sources of IL-10 in sick surgical patients include neutrophils and T-cells that are polarized towards a Th2 phenotype, which would explain this difference ${ }^{135,136}$. Timing is a critical consideration. Our results indicate that monocytes in culture increase IL-10 levels over time in regardless of conditions. At 17 hours, fresh media is used to determine the responsiveness, which is obviously not the case with the whole blood $100 \mathrm{ng} / \mathrm{mL}$ LPS challenge. This could mask the increased IL-10 levels.

There are numerous limitations to this study. This thesis demonstrates a high degree of variability in monocyte function from person to person in healthy volunteers. In addition, there is considerable clinical variability 
between these patients. There is variability in the source and severity of infection, in co-morbidity and treatments and critically, there were no specific criteria of sampling times relative to the diagnosis of infection, rather these patients were studied as targets of opportunity. This study was a spontaneous experiment of surgical practice. Both initial and follow-up samples are likely to be at different times within the clinical course from patient to patient, including a few who appeared to have fully recovered. Other limitations include potential selection bias with respect to patients, differences in age between patients and healthy volunteers, and attrition bias in that every immune parameter could not be measured in every patient. This is partly due to small volumes of blood available for analysis.

Nevertheless, the patients at the time of infection had elevated levels of IRAK-M expression relative to healthy volunteers, and this elevation appeared to return towards the level of healthy volunteers after clinical recovery. These data are similar to other groups studying patients with sepsis ${ }^{137,138}$. IRAK-M is likely to be involved in a negative feedback loop, induced by stimuli such as LPS, which in turn suppress the assembly of IRAK $1 / 4$ and decrease TRAF-6, therefore suppressing the inflammatory response through IKK and NFKB inhibition (Figure 62). In fact, various groups in experimental models have shown that silencing or knockout of IRAK-M results in an abrogation of impairment with increased cytokine production ${ }^{139}$. 
Figure 62. Proposed mechanism of IRAK-M mediated suppression of monocyte function in patients with surgical infection.

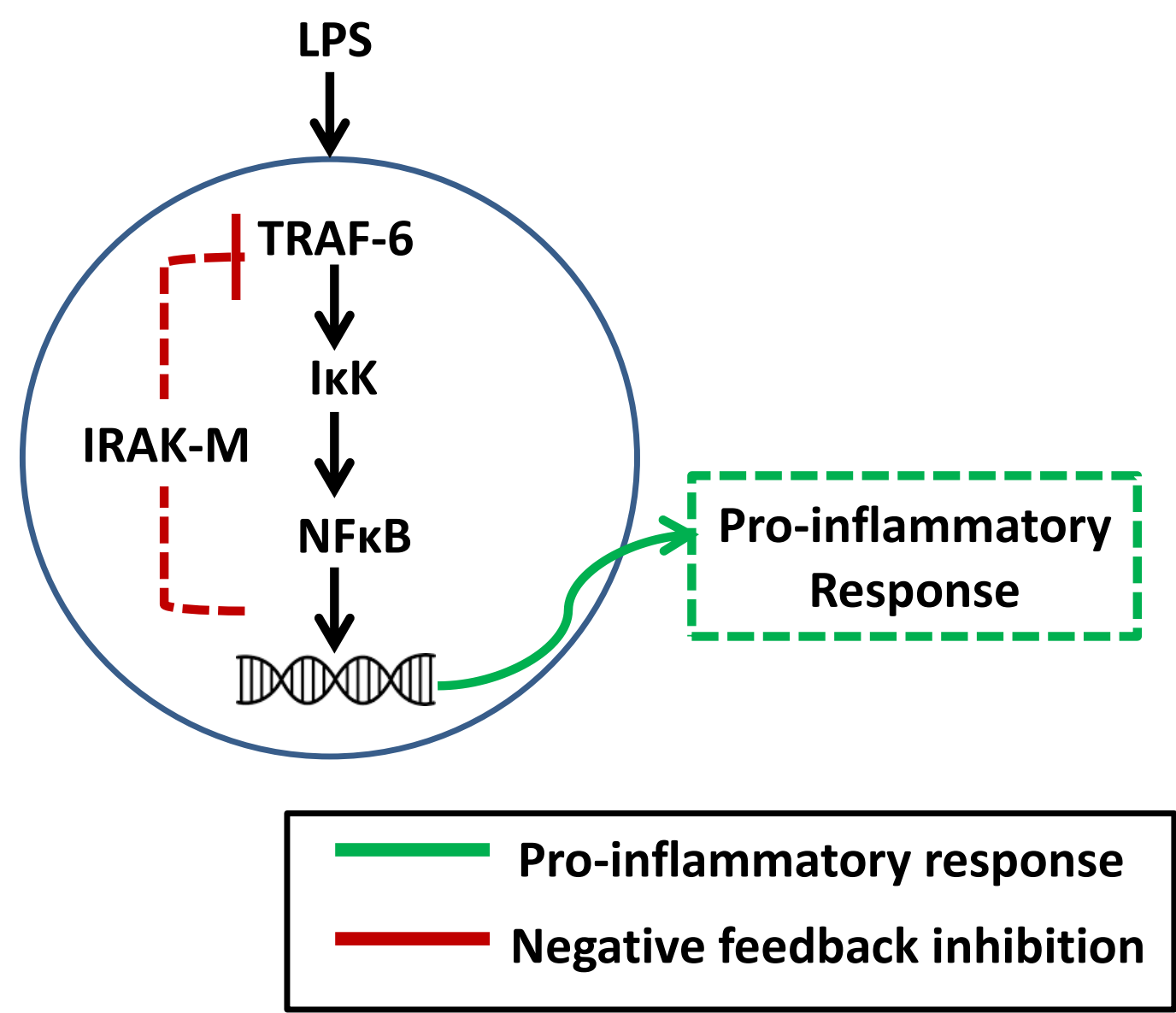

Monocytes in surgical patients will likely have initial stimulation such as from an operation, infection or soiling of the peritoneal cavity resulting in the stimulation of inflammatory pathways such as $I K K$, and resulting upregulation of IRAK-M. This negative regulator is known to suppress TLR signaling, resulting in a suppressed inflammatory response. 
In summary, this small observational study demonstrates that patients with surgical infection have impaired monocyte function, as judged by decreased TNF- $\alpha$ production in response to ex-vivo LPS stimulation and decreased HLADR expression. These results confirm to some extent the in-vitro impaired monocyte model. Upregulation of IRAK-M in infected patients may provide a potential mechanism of suppressed IKK activation and impaired responses and may be a potential marker and/or target of immunomodulation. 


\section{CHAPTER X \\ CONCLUSIONS AND OVERVIEW}

Impaired monocyte function is a central component to an immune suppression of host defense mechanisms following major trauma, surgery or infection. Although this occurs in only a subset of patients, this is predictive of poor outcomes and remains an attractive therapeutic target.

This model of isolated monocytes from healthy volunteers allows for a pure population of reproducibly impaired monocytes to be studied for underlying mechanisms and potential therapy. This is characterized by a reduced capacity to produce many cytokines and chemokines (such as TNF- $\alpha$ and IL-10), and reduced HLA-DR expression, as a result of low dose LPS exposure. This constellation of defects is representative of the patient with impaired host defenses.

The focus of this thesis has been to understand the effects of such impairment on NFKB and IKK signaling. While other pathways may also be involved, NFKB which is an important pro-inflammatory signaling pathway is indeed altered in its function in the context of monocyte impairment. Our results reflect decreased p65 protein, diminished p65 phosphorylation and decreased IKB degradation in response to a subsequent challenge, demonstrating suppressed NFKB activation in the impaired monocyte. The total levels of the IKK subunits studied do not actually change but there is 
suppressed IKK phosphorylation. This is seen most evidently within 30 minutes after the LPS challenge. This suppression of IKK activation corresponds with the decreased TNF- $\alpha$ and IL-10 production as a result of impairment. This defect is likely to be a result of a negative feedback loop that is limiting upstream signaling pathways, where we found downregulated gene expression of TRAF-6. TRAF-6 is considered the gatekeeper of toll-like receptor signaling, and its suppression in the impaired monocyte, in part, explains how decreased IKK activation occurs.

Loss of function studies with a novel, specific IKK inhibitor demonstrated cause and effect relationship of the IKK pathway, by recapitulating the impaired monocyte as measured by decreases in TNF- $\alpha$ and IL-10 production. This occurred in a dose-dependent fashion, proving the hypothesis that decreased IKK activity leads to impaired monocyte function. Interestingly, HLA-DR expression was not influenced by IKK inhibition, and is likely to be governed by other altered signaling pathways. Interestingly, miRNA-155 appeared to increase in response to LPS, but decreased with IKK inhibition. This negative effect of IKK inhibition on miRNA-155 may provide an additional mechanism to limit early inflammation, and perhaps late immunosuppression, which may offer clinical benefit. Early inhibition of IKK may be useful in some clinical settings, such as major trauma and shock, by limiting an excessive cytokine and chemokine response which is also associated with poor outcomes, without decreasing HLA-DR expression. However, it is likely that profound or prolonged IkK inhibition would likely be detrimental in a sick surgical patient. 
Understanding how our findings correspond with in-vivo monocyte impairment would be worthy of further study. One limitation of the study was that while single cell suspension allows focused mechanistic and signaling studies, the interaction of monocytes with other cells and organs in an in-vivo system, such as in an animal model, could not be elucidated. However, during the time of this thesis, we did study monocyte function in surgical patients with infection. We found that they had decreased TNF- $\alpha$ and HLA-DR expression, which confirms the validity and clinical relevance of the in-vitro model. Further observations in animal models or humans following trauma to examine IKK function and downstream cytokine networks could better inform and translate these findings at a systemic physiological level. This may also aid the understanding of negative feedback loops involved in upstream signaling dynamics, and how adjuvants like IKK-16 and IFN- $\gamma$ modulate the monocyte inflammatory response in a positive way for the "high risk" surgical patient. 
Figure 63. Summary of thesis hypothesis.

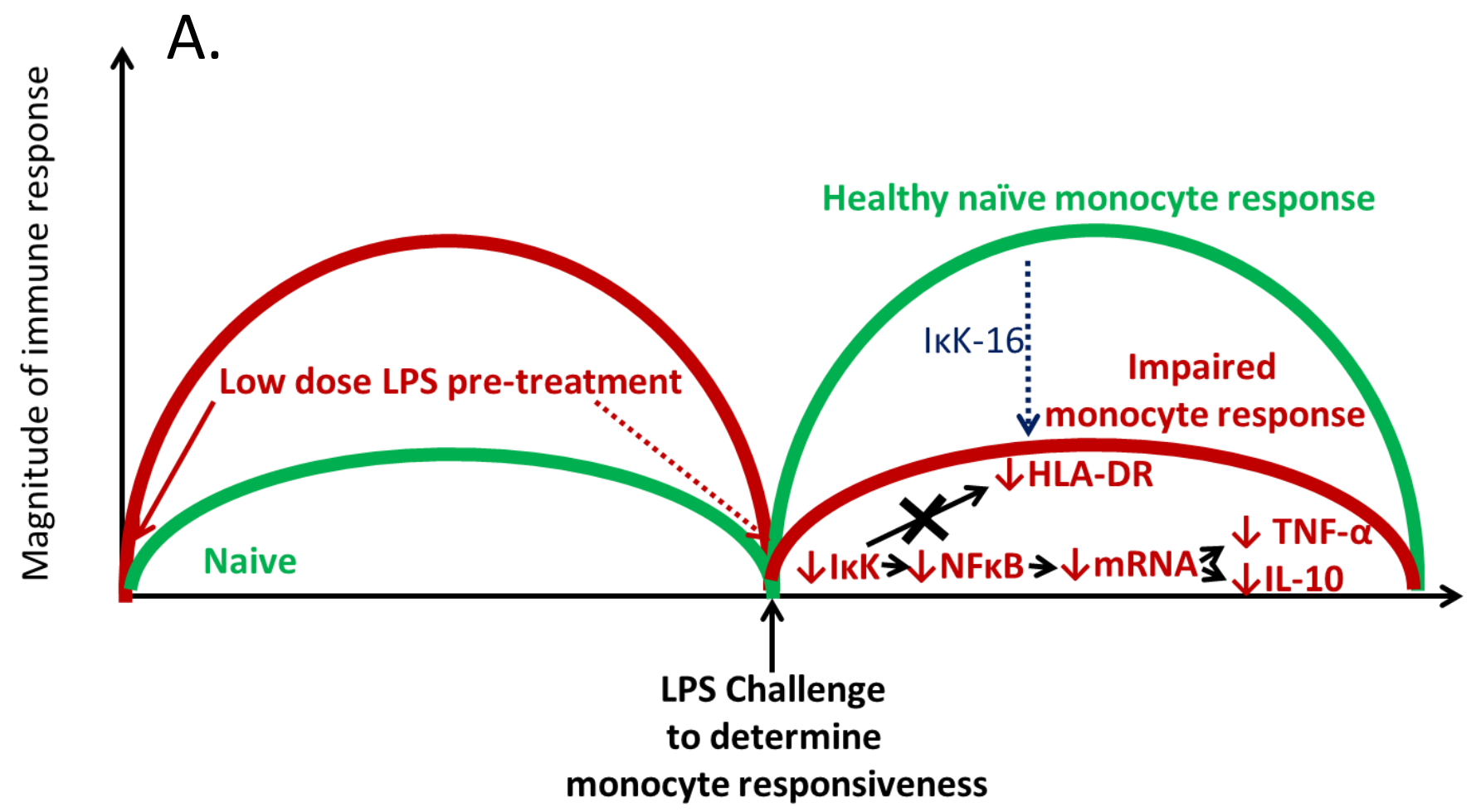

B.

Hypothesis:

LPS induced monocyte impairment occurs through sustained feedback inhibition of IKK, leading to decreased

TNF- $\alpha$ production, also represented by decreased

HLA-DR expression \& increased IL-10 production. This dissertation focuses on TNF- $\alpha$ as a valid marker of monocyte function.

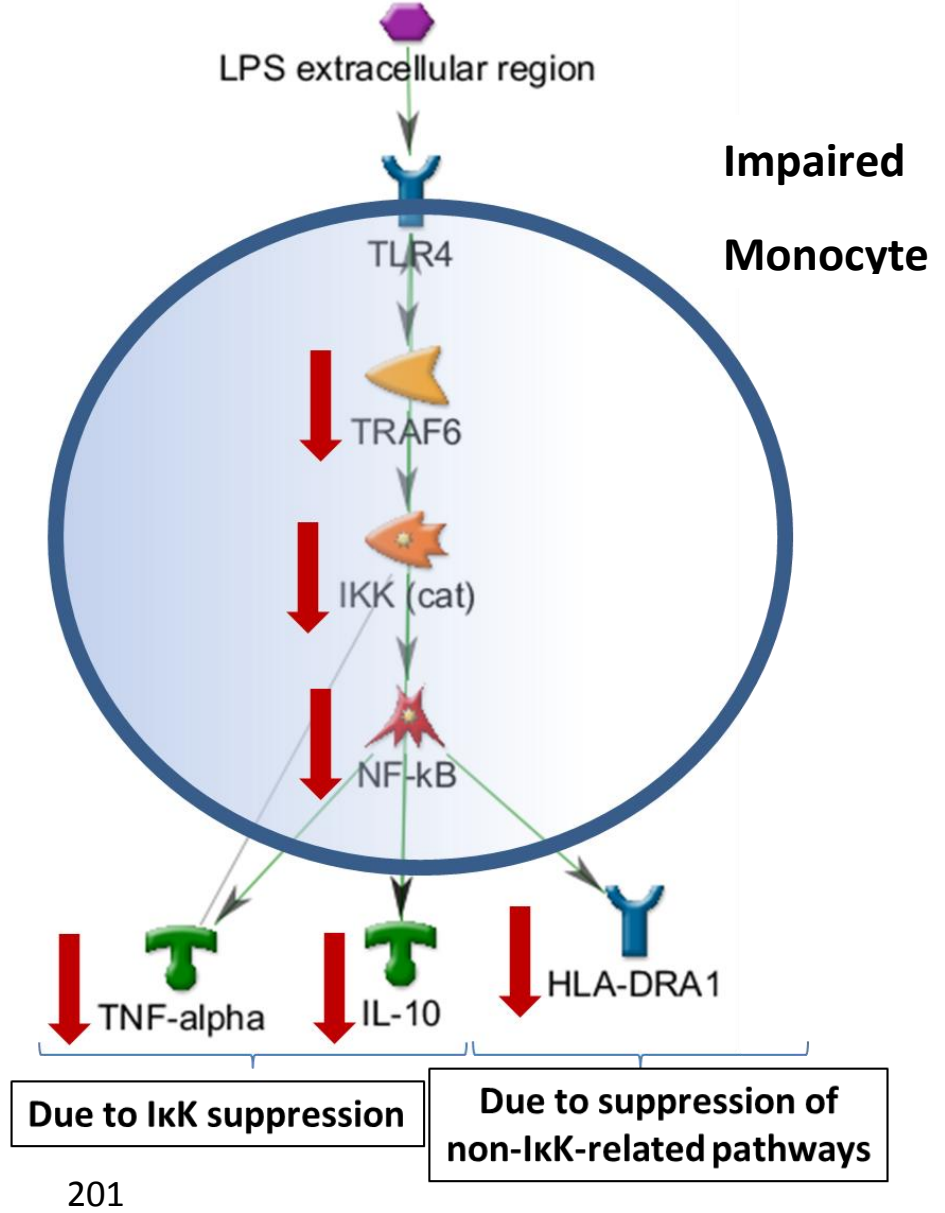




\section{Legend to Figure 63.}

We hypothesized that monocyte impairment occurred as a result of feedback inhibition of IKK. First, we developed a model of decreased monocyte function, as judged by decreased TNF- $\alpha$ and decreased IL-10 in response to an LPS challenge. Impaired monocytes also had decreased HLA-DR expression. *Decreased IL-10 production in the impaired monocyte was contrary to the hypothesis. In impaired cells previously exposed to low dose $10 \mathrm{ng} / \mathrm{mL}$ LPS, decreased IKK phosphorylation was observed. Manipulation of this pathway with an IKK inhibitor resulted in decreased TNF- $\alpha$ and IL-10 production, as seen in the impaired monocyte. HLA-DR was not decreased by IKK inhibition. Therefore, we conclude that decreased cytokine production of the impaired monocyte occurs as a result inhibition of IKK, although decreased HLA-DR likely occurs due to non-IKK related pathways. 


\section{REFERENCES}

1. Xiao $\mathrm{W}$, Mindrinos $\mathrm{MN}$, Seok J, et al. A genomic storm in critically injured humans. J Exp Med. 2011;208(13):2581-2590.

2. Ploder M, Pelinka L, Schmuckenschlager C, et al. Lipopolysaccharideinduced tumor necrosis factor alpha production and not monocyte human leukocyte antigen-DR expression is correlated with survival in septic trauma patients. Shock (Augusta, Ga.). 2006;25(2):129-134.

3. Docke WD, Randow F, Syrbe U, et al. Monocyte deactivation in septic patients: restoration by IFN-gamma treatment. Nat Med. 1997;3(6):678681.

4. Polk HC, Jr., George CD, Wellhausen SR, et al. A systematic study of host defense processes in badly injured patients. Annals of surgery. 1986;204(3):282-299 .

5. Biswas SK, Lopez-Collazo E. Endotoxin tolerance: new mechanisms, molecules and clinical significance. Trends Immunol. 2009;30(10):475487.

6. Adib-Conquy M, Asehnoune K, Moine P, Cavaillon JM. Long-termimpaired expression of nuclear factor-kappa B and I kappa B alpha in peripheral blood mononuclear cells of trauma patients. Journal of leukocyte biology. 2001;70(1):30-38.

7. Cavaillon JM, Adib-Conquy M. Bench-to-bedside review: endotoxin tolerance as a model of leukocyte reprogramming in sepsis. Critical care (London, England). 2006;10(5):233. 
8. Galbraith N, Walker S, Galandiuk S, Gardner S, Polk HC, Jr. The Significance and Challenges of Monocyte Impairment: For the III Patient and the Surgeon. Surgical infections. 2016;17(3):303-312.

9. Galbraith N, Walker S, Carter J, Polk HC, Jr. Past, Present, and Future of Augmentation of Monocyte Function in the Surgical Patient. Surgical infections. 2016.

10. Krezalek MA, DeFazio J, Zaborina O, Zaborin A, Alverdy JC. The Shift of an Intestinal "Microbiome" to a "Pathobiome" Governs the Course and Outcome of Sepsis Following Surgical Injury. Shock (Augusta, Ga.). $2016 ; 45(5): 475-482$.

11. Trunkey DD. Trauma. Accidental and intentional injuries account for more years of life lost in the U.S. than cancer and heart disease. Among the prescribed remedies are improved preventive efforts, speedier surgery and further research. Sci Am. 1983;249(2):28-35.

12. Fry DE, Pearlstein L, Fulton RL, Polk HC, Jr. Multiple system organ failure. The role of uncontrolled infection. Arch Surg. 1980;115(2):136-140. 13. Faist E, Baue AE, Dittmer H, Heberer G. Multiple organ failure in polytrauma patients. The Journal of trauma. 1983;23(9):775-787.

14. Demetriades D, Kimbrell B, Salim A, et al. Trauma deaths in a mature urban trauma system: is "trimodal" distribution a valid concept? J Am Coll Surg. 2005;201(3):343-348.

15. Hershman MJ, Cheadle WG, Wellhausen SR, Davidson PF, Polk HC, Jr. Monocyte HLA-DR antigen expression characterizes clinical outcome in the trauma patient. The British journal of surgery. 1990;77(2):204-207. 
16. Polk HC, Jr., Lamont PM, Galland RB. Containment as a mechanism of nonspecific enhancement of defenses against bacterial infection. Infect Immun. 1990;58(6):1807-1811.

17. Ziegler-Heitbrock L, Ancuta P, Crowe S, et al. Nomenclature of monocytes and dendritic cells in blood. Blood. 2010;116(16):e74-80.

18. Ziegler-Heitbrock L, Hofer TP. Toward a refined definition of monocyte subsets. Frontiers in immunology. 2013;4:23.

19. Faist E, Kupper TS, Baker CC, Chaudry IH, Dwyer J, Baue AE. Depression of cellular immunity after major injury. Its association with posttraumatic complications and its reversal with immunomodulation. Arch Surg. 1986;121(9):1000-1005.

20. Monneret G, Lepape A, Voirin N, et al. Persisting low monocyte human leukocyte antigen-DR expression predicts mortality in septic shock. Intensive Care Med. 2006;32(8):1175-1183.

21. Venet F, Tissot S, Debard AL, et al. Decreased monocyte human leukocyte antigen-DR expression after severe burn injury: Correlation with severity and secondary septic shock. Crit Care Med. 2007;35(8):19101917.

22. Lahiri R, Derwa Y, Bashir Z, et al. Systemic Inflammatory Response Syndrome After Major Abdominal Surgery Predicted by Early Upregulation of TLR4 and TLR5. Annals of surgery. 2015.

23. Cheadle WG, Hershman MJ, Wellhausen SR, Polk HC, Jr. HLA-DR antigen expression on peripheral blood monocytes correlates with surgical infection. Am J Surg. 1991;161(6):639-645. 
24. Lin ZQ, Guo J, Xia Q, et al. Human leukocyte antigen-DR expression on peripheral monocytes may be an early marker for secondary infection in severe acute pancreatitis. Hepatogastroenterology. 2013;60(128):18961902.

25. Gaudilliere B, Fragiadakis GK, Bruggner RV, et al. Clinical recovery from surgery correlates with single-cell immune signatures. Sci Transl Med. 2014;6(255):255ra131.

26. Longbottom ER, Torrance HD, Owen HC, et al. Features of Postoperative Immune Suppression Are Reversible With Interferon Gamma and Independent of Interleukin-6 Pathways. Annals of surgery. 2016;264(2):370-377.

27. Lyons A, Kelly JL, Rodrick ML, Mannick JA, Lederer JA. Major injury induces increased production of interleukin- 10 by cells of the immune system with a negative impact on resistance to infection. Annals of surgery. 1997;226(4):450-458; discussion 458-460.

28. Stensballe J, Christiansen M, Tonnesen E, Espersen K, Lippert FK, Rasmussen LS. The early IL- 6 and IL-10 response in trauma is correlated with injury severity and mortality. Acta Anaesthesiol Scand. 2009;53(4):515-521.

29. Hoffman JA, Weinberg KI, Azen CG, et al. Human leukocyte antigenDR expression on peripheral blood monocytes and the risk of pneumonia in pediatric lung transplant recipients. Transp/ Infect Dis. 2004;6(4):147155. 
30. Handy JM, Scott AJ, Cross AM, Sinha P, O'Dea KP, Takata M. HLADR expression and differential trafficking of monocyte subsets following low to intermediate risk surgery. Anaesthesia. 2010;65(1):27-35.

31. Biffl WL, Moore EE, Moore FA, Peterson VM. Interleukin-6 in the injured patient. Marker of injury or mediator of inflammation? Annals of surgery. 1996;224(5):647-664.

32. Gouel-Cheron A, Allaouchiche B, Guignant C, et al. Early interleukin-6 and slope of monocyte human leukocyte antigen-DR: a powerful association to predict the development of sepsis after major trauma. PloS one. $2012 ; 7(3): \mathrm{e} 33095$.

33. Guignant C, Lepape A, Huang X, et al. Programmed death-1 levels correlate with increased mortality, nosocomial infection and immune dysfunctions in septic shock patients. Critical care (London, England). $2011 ; 15(2): R 99$.

34. Hietbrink F, Koenderman L, van Wessem KJ, Leenen LP. The Impact of Intramedullary Nailing of Tibia Fractures on the Innate Immune System. Shock (Augusta, Ga.). 2015;44(3):209-214.

35. Kirchhoff C, Biberthaler P, Mutschler WE, Faist E, Jochum M, Zedler S. Early down-regulation of the pro-inflammatory potential of monocytes is correlated to organ dysfunction in patients after severe multiple injury: a cohort study. Critical care (London, England). 2009;13(3):R88.

36. Sfeir T, Saha DC, Astiz M, Rackow EC. Role of interleukin-10 in monocyte hyporesponsiveness associated with septic shock. Crit Care Med. 2001;29(1):129-133. 
37. Laudanski K, Miller-Graziano C, Xiao W, et al. Cell-specific expression and pathway analyses reveal alterations in trauma-related human $\mathrm{T}$ cell and monocyte pathways. Proceedings of the National Academy of Sciences of the United States of America. 2006;103(42):15564-15569. 38. Thompson CM, Park CH, Maier RV, O'Keefe GE. Traumatic injury, early gene expression, and gram-negative bacteremia. Crit Care Med. 2014;42(6):1397-1405.

39. Namas RA, Vodovotz Y, Almahmoud K, et al. Temporal Patterns of Circulating Inflammation Biomarker Networks Differentiate Susceptibility to Nosocomial Infection Following Blunt Trauma in Humans. Annals of surgery. 2014.

40. Almahmoud K, Namas RA, Abdul-Malak O, et al. Impact of Injury Severity on Dynamic Inflammation Networks Following Blunt Trauma. Shock (Augusta, Ga.). 2015;44(2):101-109.

41. Asadullah K, Woiciechowsky C, Docke WD, et al. Very low monocytic HLA-DR expression indicates high risk of infection--immunomonitoring for patients after neurosurgery and patients during high dose steroid therapy. Eur J Emerg Med. 1995;2(4):184-190.

42. van den Berk JM, Oldenburger $\mathrm{RH}$, van den Berg AP, et al. Low HLADR expression on monocytes as a prognostic marker for bacterial sepsis after liver transplantation. Transplantation. 1997;63(12):1846-1848.

43. Oczenski W, Krenn H, Jilch R, et al. HLA-DR as a marker for increased risk for systemic inflammation and septic complications after cardiac surgery. Intensive Care Med. 2003;29(8):1253-1257. 
44. Albertsmeier M, Quaiser D, von Dossow-Hanfstingl V, Winter H, Faist E, Angele MK. Major surgical trauma differentially affects T-cells and APC. Innate Immun. 2015;21(1):55-64.

45. Cajander S, Backman A, Tina E, Stralin K, Soderquist B, Kallman J. Preliminary results in quantitation of HLA-DRA by real-time PCR: a promising approach to identify immunosuppression in sepsis. Critical care (London, England). 2013;17(5):R223.

46. Turrel-Davin F, Venet F, Monnin C, et al. mRNA-based approach to monitor recombinant gamma-interferon restoration of LPS-induced endotoxin tolerance. Critical care (London, England). 2011;15(5):R252. 47. Allantaz-Frager F, Turrel-Davin F, Venet F, et al. Identification of biomarkers of response to IFNg during endotoxin tolerance: application to septic shock. PloS one. 2013;8(7):e68218.

48. Fontaine M, Planel S, Peronnet E, et al. S100A8/A9 mRNA induction in an ex vivo model of endotoxin tolerance: roles of IL-10 and IFNgamma. PloS one. 2014;9(6):e100909.

49. Hotchkiss RS, Monneret G, Payen D. Immunosuppression in sepsis: a novel understanding of the disorder and a new therapeutic approach. Lancet Infect Dis. 2013;13(3):260-268.

50. Zhang Y, Zhou Y, Lou J, et al. PD-L1 blockade improves survival in experimental sepsis by inhibiting lymphocyte apoptosis and reversing monocyte dysfunction. Critical care (London, England). 2010;14(6):R220. 51.Zhu W, Bao R, Fan X, et al. PD-L1 blockade attenuated sepsis-induced liver injury in a mouse cecal ligation and puncture model. Mediators of inflammation. 2013;2013:361501. 
52. Monaghan SF, Thakkar RK, Tran ML, et al. Programmed death 1 expression as a marker for immune and physiological dysfunction in the critically ill surgical patient. Shock (Augusta, Ga.). 2012;38(2):117-122. 53. Mansur A, Hinz J, Hillebrecht B, et al. Ninety-day survival rate of patients with sepsis relates to programmed cell death 1 genetic polymorphism rs11568821. J Investig Med. 2014;62(3):638-643.

54. Billeter AT, Hellmann J, Roberts $\mathrm{H}$, et al. MicroRNA-155 potentiates the inflammatory response in hypothermia by suppressing IL-10 production. FASEB journal : official publication of the Federation of American Societies for Experimental Biology. 2014;28(12):5322-5336. 55. Qadan M, Weller EB, Gardner SA, Maldonado C, Fry DE, Polk HC, Jr. Glucose and surgical sepsis: a study of underlying immunologic mechanisms. J Am Coll Surg. 2010;210(6):966-974.

56. Qadan M, Battista C, Gardner SA, Anderson G, Akca O, Polk HC, Jr. Oxygen and surgical site infection: a study of underlying immunologic mechanisms. Anesthesiology. 2010;113(2):369-377.

57. Muszynski JA, Frazier E, Nofziger R, et al. Red blood cell transfusion and immune function in critically ill children: a prospective observational study. Transfusion. 2014.

58. Muszynski JA, Bale J, Nateri J, et al. Supernatants from stored red blood cell (RBC) units, but not RBC-derived microvesicles, suppress monocyte function in vitro. Transfusion. 2015;55(8):1937-1945.

59. Veenhof AA, Sietses C, von Blomberg BM, et al. The surgical stress response and postoperative immune function after laparoscopic or 
conventional total mesorectal excision in rectal cancer: a randomized trial. Int J Colorectal Dis. 2011;26(1):53-59.

60. Shibata J, Ishihara S, Tada N, et al. Surgical stress response after colorectal resection: a comparison of robotic, laparoscopic, and open surgery. Tech Coloproctol. 2015;19(5):275-280.

61. Chopra SS, Haacke N, Meisel C, Unterwalder N, Fikatas P, Schmidt SC. Postoperative immunosuppression after open and laparoscopic liver resection: assessment of cellular immune function and monocytic HLA-DR expression. JSLS. 2013;17(4):615-621.

62. Sista F, Schietroma M, Santis GD, et al. Systemic inflammation and immune response after laparotomy vs laparoscopy in patients with acute cholecystitis, complicated by peritonitis. World J Gastrointest Surg. 2013;5(4):73-82.

63. Veenhof AA, Vlug MS, van der Pas MH, et al. Surgical stress response and postoperative immune function after laparoscopy or open surgery with fast track or standard perioperative care: a randomized trial. Annals of surgery. 2012;255(2):216-221.

64. Fujita $T$. Interpreting postoperative CRP response and HLA-DR depression. Annals of surgery. 2015;261(2):e39.

65. Beeson PB, Technical Assistance of Elizabeth R. Tolerance to Bacterial Pyrogens : I. Factors Influencing Its Development. J Exp Med. 1947;86(1):29-38.

66. Draisma A, Pickkers P, Bouw MP, van der Hoeven JG. Development of endotoxin tolerance in humans in vivo. Crit Care Med. 2009;37(4):12611267. 
67. Wolk K, Docke WD, von Baehr V, Volk HD, Sabat R. Impaired antigen presentation by human monocytes during endotoxin tolerance. Blood. 2000;96(1):218-223.

68. del Fresno C, Garcia-Rio F, Gomez-Pina V, et al. Potent phagocytic activity with impaired antigen presentation identifying lipopolysaccharidetolerant human monocytes: demonstration in isolated monocytes from cystic fibrosis patients. Journal of immunology (Baltimore, Md. : 1950). 2009;182(10):6494-6507.

69. Murphy M, Xiong Y, Pattabiraman G, Qiu F, Medvedev AE. Pellino-1 Positively Regulates Toll-like Receptor (TLR) 2 and TLR4 Signaling and Is Suppressed upon Induction of Endotoxin Tolerance. J Biol Chem. 2015;290(31):19218-19232.

70. Xiong Y, Qiu F, Piao W, Song C, Wahl LM, Medvedev AE. Endotoxin tolerance impairs IL-1 receptor-associated kinase (IRAK) 4 and TGF-betaactivated kinase 1 activation, K63-linked polyubiquitination and assembly of IRAK1, TNF receptor-associated factor 6, and IkappaB kinase gamma and increases A20 expression. J Biol Chem. 2011;286(10):7905-7916. 71. Ziegler-Heitbrock L. The p50-homodimer mechanism in tolerance to LPS. J Endotoxin Res. 2001;7(3):219-222.

72. Amaya M, Keck F, Bailey C, Narayanan A. The role of the IKK complex in viral infections. Pathogens and disease. 2014;72(1):32-44.

73. Hinz M, Scheidereit C. The IkappaB kinase complex in NF-kappaB regulation and beyond. EMBO reports. 2014;15(1):46-61. 
74. Luo JL, Kamata H, Karin M. IKK/NF-kappaB signaling: balancing life and death--a new approach to cancer therapy. The Journal of clinical investigation. 2005;115(10):2625-2632.

75. Williams DL, Ha T, Li C, Kalbfleisch JH, Ferguson DA, Jr. Early activation of hepatic NFkappaB and NF-IL6 in polymicrobial sepsis correlates with bacteremia, cytokine expression, and mortality. Annals of surgery. 1999;230(1):95-104.

76. Lahiri R, Derwa Y, Bashir Z, et al. Systemic Inflammatory Response Syndrome After Major Abdominal Surgery Predicted by Early Upregulation of TLR4 and TLR5. Annals of surgery. 2016;263(5):1028-1037.

77. Sordi R, Chiazza F, Johnson FL, et al. Inhibition of IkappaB Kinase Attenuates the Organ Injury and Dysfunction Associated with Hemorrhagic Shock. Molecular medicine (Cambridge, Mass.). 2015;21:563-575. 78. Coldewey SM, Rogazzo M, Collino M, Patel NS, Thiemermann C. Inhibition of IkappaB kinase reduces the multiple organ dysfunction caused by sepsis in the mouse. Disease models \& mechanisms. 2013;6(4):1031-1042.

79. Shu YS, Tao W, Miao QB, Zhu YB, Yang YF. Improvement of ventilation-induced lung injury in a rodent model by inhibition of inhibitory kappaB kinase. The journal of trauma and acute care surgery. $2014 ; 76(6): 1417-1424$.

80. Chen J, Kieswich JE, Chiazza F, et al. IkappaB Kinase Inhibitor Attenuates Sepsis-Induced Cardiac Dysfunction in CKD. Journal of the American Society of Nephrology : JASN. 2016. 
81. Li H, Han W, Polosukhin V, et al. NF-kappaB inhibition after cecal ligation and puncture reduces sepsis-associated lung injury without altering bacterial host defense. Mediators of inflammation. 2013;2013:503213.

82. Docke WD, Hoflich C, Davis KA, et al. Monitoring temporary immunodepression by flow cytometric measurement of monocytic HLA-DR expression: a multicenter standardized study. Clinical chemistry. $2005 ; 51(12): 2341-2347$.

83. Demaret J, Walencik A, Jacob MC, et al. Inter-laboratory assessment of flow cytometric monocyte HLA-DR expression in clinical samples. Cytometry. Part B, Clinical cytometry. 2013;84(1):59-62.

84. Dries DJ, Jurkovich GJ, Maier RV, et al. Effect of interferon gamma on infection-related death in patients with severe injuries. A randomized, double-blind, placebo-controlled trial. Arch Surg. 1994;129(10):1031-1041; discussion 1042.

85. Polk HC, Jr., Cheadle WG, Livingston DH, et al. A randomized prospective clinical trial to determine the efficacy of interferon-gamma in severely injured patients. Am J Surg. 1992;163(2):191-196.

86. Leentjens J, Kox M, Koch RM, et al. Reversal of immunoparalysis in humans in vivo: a double-blind, placebo-controlled, randomized pilot study. American journal of respiratory and critical care medicine. 2012;186(9):838-845.

87. Turina M, Dickinson A, Gardner S, Polk HC, Jr. Monocyte HLA-DR and interferon-gamma treatment in severely injured patients--a critical reappraisal more than a decade later. J Am Coll Surg. 2006;203(1):73-81. 
88. Bone RC, Balk RA, Cerra FB, et al. Definitions for sepsis and organ failure and guidelines for the use of innovative therapies in sepsis. The ACCP/SCCM Consensus Conference Committee. American College of Chest Physicians/Society of Critical Care Medicine. Chest. 1992;101(6):1644-1655.

89. Wasserman D, loannovich JD, Hinzmann RD, Deichsel G, Steinmann GG. Interferon-gamma in the prevention of severe burn-related infections: a European phase III multicenter trial. The Severe Burns Study Group. Critical care medicine. 1998;26(3):434-439.

90. Nakos G, Malamou-Mitsi VD, Lachana A, et al. Immunoparalysis in patients with severe trauma and the effect of inhaled interferon-gamma. Crit Care Med. 2002;30(7):1488-1494.

91. Schinkel C, Licht K, Zedler S, Schinkel S, Fuchs D, Faist E. Perioperative treatment with human recombinant interferon-gamma: a randomized double-blind clinical trial. Shock (Augusta, Ga.). 2001;16(5):329-333.

92. Licht AK, Schinkel C, Zedler S, Schinkel S, Faist E. Effects of perioperative recombinant human IFN-gamma (rHulFN-gamma) application in vivo on T cell response. J Interferon Cytokine Res. 2003;23(3):149-154.

93. Meisel C, Schefold JC, Pschowski R, et al. Granulocyte-macrophage colony-stimulating factor to reverse sepsis-associated immunosuppression: a double-blind, randomized, placebo-controlled multicenter trial. American journal of respiratory and critical care medicine. 2009;180(7):640-648. 
94.Spies C, Luetz A, Lachmann G, et al. Influence of GranulocyteMacrophage Colony-Stimulating Factor or Influenza Vaccination on HLADR, Infection and Delirium Days in Immunosuppressed Surgical Patients: Double Blind, Randomised Controlled Trial. PloS one. 2015;10(12):e0144003.

95. Schneider C, von Aulock S, Zedler S, Schinkel C, Hartung T, Faist E. Perioperative recombinant human granulocyte colony-stimulating factor (Filgrastim) treatment prevents immunoinflammatory dysfunction associated with major surgery. Annals of surgery. 2004;239(1):75-81. 96. Bo L, Wang F, Zhu J, Li J, Deng X. Granulocyte-colony stimulating factor (G-CSF) and granulocyte-macrophage colony stimulating factor (GM-CSF) for sepsis: a meta-analysis. Critical care (London, England). $2011 ; 15(1): R 58$

97. Cheadle WG, Hanasawa K, Gallinaro RN, Nimmanwudipong T, Kodama M, Polk HC, Jr. Endotoxin filtration and immune stimulation improve survival from gram-negative sepsis. Surgery. 1991;110(4):785791; discussion 791-782.

98. Esteban E, Ferrer R, Alsina L, Artigas A. Immunomodulation in sepsis: the role of endotoxin removal by polymyxin B-immobilized cartridge. Mediators of inflammation. 2013;2013:507539.

99. Ranieri VM, Thompson BT, Barie PS, et al. Drotrecogin alfa (activated) in adults with septic shock. N Engl J Med. 2012;366(22):2055-2064. 100. Polk HC, Jr., Lopez-Mayor JF. Postoperative wound infection: a prospective study of determinant factors and prevention. Surgery. 1969;66(1):97-103. 
101. Marshall JC. Why have clinical trials in sepsis failed? Trends Mol Med. 2014;20(4):195-203.

102. Opal SM, Laterre PF, Francois B, et al. Effect of eritoran, an antagonist of MD2-TLR4, on mortality in patients with severe sepsis: the ACCESS randomized trial. JAMA. 2013;309(11):1154-1162.

103. Hotchkiss RS, Monneret G, Payen D. Sepsis-induced immunosuppression: from cellular dysfunctions to immunotherapy. Nat Rev Immunol. 2013;13(12):862-874.

104. Zhou J, Chaudhry H, Zhong Y, et al. Dysregulation in microRNA expression in peripheral blood mononuclear cells of sepsis patients is associated with immunopathology. Cytokine. 2015;71(1):89-100. 105. Wang HJ, Zhang PJ, Chen WJ, et al. Characterization and Identification of novel serum microRNAs in sepsis patients with different outcomes. Shock (Augusta, Ga.). 2013;39(6):480-487.

106. Wang $H$, Zhang $P$, Chen W, Feng D, Jia Y, Xie L. Serum microRNA signatures identified by Solexa sequencing predict sepsis patients' mortality: a prospective observational study. PloS one. 2012;7(6):e38885.

107. Wang HJ, Zhang PJ, Chen WJ, Feng D, Jia YH, Xie LX. Four serum microRNAs identified as diagnostic biomarkers of sepsis. The journal of trauma and acute care surgery. 2012;73(4):850-854.

108. Uhlich RM, Konie JA, Davis JW, et al. Novel microRNA correlations in the severely injured. Surgery. 2014;156(4):834-840. 109. Owen HC, Torrance HD, Jones TF, et al. Epigenetic regulatory pathways involving microRNAs may modulate the host immune response 
following major trauma. The journal of trauma and acute care surgery. 2015;79(5):766-772.

110. Zhang $A Q$, Gu W, Zeng L, et al. Genetic variants of microRNA sequences and susceptibility to sepsis in patients with major blunt trauma. Annals of surgery. 2015;261(1):189-196.

111. Tacke F, Roderburg C, Benz F, et al. Levels of circulating miR133a are elevated in sepsis and predict mortality in critically ill patients. Crit Care Med. 2014;42(5):1096-1104.

112. Livak KJ, Schmittgen TD. Analysis of relative gene expression data using real-time quantitative PCR and the 2(-Delta Delta $C(T)$ ) Method. Methods (San Diego, Calif.). $2001 ; 25(4): 402-408$.

113. Warren HS, Tompkins RG, Mindrinos MN, Xiao W, Davis RW. Reply to Cauwels et al.: Of men, not mice, and inflammation. Proceedings of the National Academy of Sciences of the United States of America. 2013;110(34):E3151.

114. Tracey KJ, Fong Y, Hesse DG, et al. Anti-cachectin/TNF monoclonal antibodies prevent septic shock during lethal bacteraemia. Nature. 1987;330(6149):662-664.

115. Adib-Conquy M, Moine $\mathrm{P}$, Asehnoune $\mathrm{K}$, et al. Toll-like receptormediated tumor necrosis factor and interleukin-10 production differ during systemic inflammation. American journal of respiratory and critical care medicine. 2003;168(2):158-164.

116. Lee KW, Lee Y, Kim DS, Kwon HJ. Direct role of NF-kappaB activation in Toll-like receptor-triggered HLA-DRA expression. European journal of immunology. 2006;36(5):1254-1266. 
117. Ceppi M, Pereira PM, Dunand-Sauthier I, et al. MicroRNA-155 modulates the interleukin-1 signaling pathway in activated human monocyte-derived dendritic cells. Proceedings of the National Academy of Sciences of the United States of America. 2009;106(8):2735-2740. 118. Hochberg Y, Benjamini Y. More powerful procedures for multiple significance testing. Statistics in medicine. 1990;9(7):811-818.

119. Jung SH. Sample size for FDR-control in microarray data analysis. Bioinformatics (Oxford, England). 2005;21(14):3097-3104. 120. Namas RA, Vodovotz Y, Almahmoud K, et al. Temporal Patterns of Circulating Inflammation Biomarker Networks Differentiate Susceptibility to Nosocomial Infection Following Blunt Trauma in Humans. Annals of surgery. 2016;263(1):191-198.

121. Benjamini Y. Discovering the false discovery rate. Journal of the Royal Statistical Society: Series B (Statistical Methodology). 2010;72(4):405-416 .

122. Storey JD. A direct approach to false discovery rates. Journal of the Royal Statistical Society: Series B (Statistical Methodology). 2002;64(3):479-498.

123. Mathias B, Delmas AL, Ozrazgat-Baslanti T, et al. Human Myeloid-derived Suppressor Cells are Associated With Chronic Immune Suppression After Severe Sepsis/Septic Shock. Annals of surgery. 2016. 124. Di Paolo NC, Shayakhmetov DM. Interleukin 1alpha and the inflammatory process. Nature immunology. 2016;17(8):906-913. 
125. Ziraldo C, Vodovotz Y, Namas RA, et al. Central role for MCP1/CCL2 in injury-induced inflammation revealed by in vitro, in silico, and clinical studies. PloS one. 2013;8(12):e79804.

126. Bauer D, Redmon N, Mazzio E, et al. Diallyl disulfide inhibits TNFalpha induced CCL2 release through MAPK/ERK and NF-Kappa-B signaling. Cytokine. 2015;75(1):117-126.

127. Wang J, Vodovotz Y, Fan L, et al. Injury-induced MRP8/MRP14 stimulates IP-10/CXCL10 in monocytes/macrophages. FASEB journal : official publication of the Federation of American Societies for Experimental Biology. 2015;29(1):250-262.

128. Shi L, Song L, Maurer K, Sharp J, Zhang Z, Sullivan KE. Endotoxin tolerance in monocytes can be mitigated by alpha2-interferon. Journal of leukocyte biology. 2015;98(4):651-659.

129. Harding CV, Boom WH. Regulation of antigen presentation by Mycobacterium tuberculosis: a role for Toll-like receptors. Nature reviews. Microbiology. 2010;8(4):296-307.

130. Bellucci R, Martin A, Bommarito D, et al. Interferon-gammainduced activation of JAK1 and JAK2 suppresses tumor cell susceptibility to NK cells through upregulation of PD-L1 expression. Oncoimmunology. 2015;4(6):e1008824.

131. Carrillo EH, Gordon L, Goode E, Davis E, Polk HC, Jr. Early elevation of soluble CD14 may help identify trauma patients at high risk for infection. The Journal of trauma. 2001;50(5):810-816. 
132. Heinzelmann M, Mercer-Jones M, Cheadle WG, Polk HC, Jr. CD14 expression in injured patients correlates with outcome. Annals of surgery. 1996;224(1):91-96.

133. Rettig TC, Verwijmeren L, Dijkstra IM, Boerma D, van de Garde EM, Noordzij PG. Postoperative Interleukin-6 Level and Early Detection of Complications After Elective Major Abdominal Surgery. Annals of surgery. 2016;263(6):1207-1212.

134. Monneret G, Finck ME, Venet F, et al. The anti-inflammatory response dominates after septic shock: association of low monocyte HLADR expression and high interleukin-10 concentration. Immunology letters. 2004;95(2):193-198.

135. Noel G, Wang Q, Schwemberger S, et al. Neutrophils, not monocyte/macrophages, are the major splenic source of postburn IL-10. Shock (Augusta, Ga.). 2011;36(2):149-155.

136. Islam MN, Bradley BA, Ceredig R. Sterile post-traumatic immunosuppression. Clinical \& translational immunology. 2016;5(4):e77. 137. Escoll P, del Fresno C, Garcia L, et al. Rapid up-regulation of IRAK-M expression following a second endotoxin challenge in human monocytes and in monocytes isolated from septic patients. Biochemical and biophysical research communications. 2003;311(2):465-472.

138. van 't Veer C, van den Pangaart PS, van Zoelen MA, et al. Induction of IRAK-M is associated with lipopolysaccharide tolerance in a human endotoxemia model. Journal of immunology (Baltimore, Md. : 1950). 2007;179(10):7110-7120. 
139. Kobayashi K, Hernandez LD, Galan JE, Janeway CA, Jr., Medzhitov R, Flavell RA. IRAK-M is a negative regulator of Toll-like receptor signaling. Cell. 2002;110(2):191-202. 


\section{CURRICULUM VITAE}

Dr. Norman Galbraith

BSc (Med Sci), MBChB, MSc, PhD, MRCS

1639 Jaeger Avenue

Louisville, KY 40205

Cell: +1 5029300675

Email: norman.galbraith@louisville.edu

\section{Undergraduate Qualifications}

Bachelor of Medicine \& Bachelor of Surgery (MBChB)

Faculty of Medicine, University of Glasgow

Final Examination, Clinical Medicine:

Written Paper: A (Excellent)

OSCE: A (Excellent)

Bachelor of Science (BSc Med Sci) in Anatomical Sciences

Faculty of Biological and Life Sciences, University of Glasgow

This intercalated degree consisted of modules ranging from molecular methods to clinical anatomy, combined with academic teaching on critical analysis and statistics.

Research Project: The Importance of Blood Vessels in Laparoscopic Adrenalectomy Supervisor: $\quad$ Professor Patrick O’Dwyer, Department of Surgery, Western Infirmary

This involved a literature review, attending surgical procedures, using original clinical data, and employing traditional anatomical techniques. Achieved Upper Second Class (2.1) Honours.

\section{Postgraduate Qualifications}

$\operatorname{MRCS}(\mathrm{Ed})$

Intercollegiate

Royal College of Surgeons, Edinburgh

Masters in Surgical Sciences (MSc)

Edinburgh Surgical Sciences Qualification (ESSQ)

Part time distance learning degree

Dissertation: "MBL and the acute phase response: A factor in post-operative infection following liver resection?"

Achieved Distinction

University of Edinburgh/Royal College of Surgeons Edimburgh.

Doctor of Philosophy in Physiology (PhD)

$2014-2016$

Involved 2.5 years of bench research

Formal PhD level physiology classes: Statistics, integrated systemic physiology, advanced cardiovascular physiology

Dissertation: "The role and regulation of Inhibitor of кB Kinase (ІкК) in the impaired monocyte response" Department of Physiology \& Biophysics, University of Louisville School of Medicine

\section{Prizes \& Awards}

Distinction and Commendation

Clinical Medicine, May 2010, MBChB, University of Glasgow 


\section{Distinction}

Diploma (Year 2, 2012) \& Masters (Year 3, 2013)

MSc, Edinburgh Surgical Sciences Qualification (ESSQ),

University of Edinburgh/RCSEd

\section{Graduate Deans Citation}

Awarded to $<10 \%$ of graduates from the School of Graduate Studies in recognition of superior accomplishment beyond maintaining a high grade point average (GPA), Winter 2016

School of Interdisciplinary and Graduate Studies, University of Louisville

\section{Representative of the School of Medicine as the Banner Bearer}

Winter Graduation Commencement, 2016

University of Louisville School of Medicine

\section{Employment}

Ferguson Research Fellowship

Price Institute of Surgical Research, Department of Surgery, University of Louisville, Kentucky, US A.

Supervisor: Hiram C. Polk, Jr. MD FACS

Role involving literature research, experimental design, executing experiments, data analysis and presentation.

Techniques included monocyte isolation, cell culture, ELISA \& multiplex protein analysis, qPCR, Flow cytometry, Western Blot, Immunofluorescence microscopy.

Supervision of high school, undergraduate and medical students in mentoring and teaching scientific technique.

Additional skills developed in submitting academic grants, national and international presentations of research and manuscript preparation and submission to peer reviewed journals. As a member of the Department of Surgery, this involved regular attendance at weekly teaching trauma rounds, grand rounds, morbidity and mortality meetings and examining medical student surgical exams.

Core Surgical Training

6 month rotations in:

General Surgery, Western Isles Hospital, Stornoway, Isle of Lewis

ENT Surgery, St John's Hospital, Livingstone

Colorectal Surgery, Western General Hospital, Edinburgh

Urology, Western General Hospital, Edinburgh

Academic Foundation Programme

$2010-2012$

South East Scotland Foundation School

\section{Academic Supervisor:}

Professor Stephen Wigmore, Professor of Transplantation, University of Edinburgh

FY1 rotations: General Medicine (BGH), Acute Medicine (WGH), Colorectal Surgery (WGH)

FY2 rotations: Gastroenterology (WGH), General Surgery (RIE), Orthopaedic Surgery (RIE) 


\section{Professional Memberships}

- $\quad$ Society for Surgery of the Alimentary Tract (SSAT) 2016

- $\quad$ Surgical Infection Society (SIS) 2016

- Association of Surgeons in Training (ASiT) 2014

- $\quad$ Royal College of Surgeons, Edinburgh 2013

- General Medical Council (GMC) 2010

\section{Courses and Meetings Attended}

- Academic Surgical Congress, Las Vegas, 2017 (Registered)

- Surgical Infection Society Annual Meeting, West Palm Beach, 2016

- Academic Surgical Congress, Jacksonville, FL, 2016

- Academic Surgical Congress, Las Vegas, 2015

- $\quad$ ASC Grant Writing Course, ASC Congress, Las Vegas, 2015

- Train the Trainers, Royal College of Surgeons England, 2013

- Care of the Critically Ill Surgical Patient (CCrISP), Liverpool, 2013

- ATLS, Raigmore Hospital, Inverness. 2012

- $\quad$ ALS, $17^{\text {th }}$ Jan 2012

- Basic Surgical Skills, Royal College of Physicians and Surgeons, Glasgow, 2011

- $\quad$ ILS, University of Glasgow, 2010.

\section{Audit}

- Fluid prescription in Surgical Patients by Junior Doctors

Working with Mr Hugh Paterson, Consultant Colorectal Surgeon, Western General Hospital, 2012.

An audit consisting of surveying FY1 doctors on their fluid prescription regimes in various scenarios in combination with clinical data of fluid prescription in surgical patients. Resulted in regular fluid teaching program, with re-audit complete.

- Audit of Compliance with VTE Prophylaxis including Reassessment in WIH Western Isles Hospital, Stornoway, Isle of Lewis, 2012.

Conceptualised, designed, collected data for \& presented at a local meeting. Resulted in a change of practice, with new documentation and the second cycle is about to begin.

- Audit of Surgical Management of Abscesses in the Colorectal Unit.

Western General Hospital, Dec 2013.

Audit of time/inpatient stay from admission to drainage, and whether this was undertaken "out of hours", presented to the regional Colorectal Morbidity \& Mortality meeting.

- Audit of Risk assessment \& Appropriate Heparin/TED prescription in the Urology Unit, WGH.

Western General Hospital, Edinburgh, 2014

I supervised an FY1 doctor in the design, collection and data analysis. We presented the first cycle, with a view to implementing an intervention through stickers and for re-audit.

- Loop ileostomy patient education in an American Colorectal Unit. University Hospital, Louisville, Kentucky, 2016.

An initial audit revealed a high rate of readmission following loop ileostomy formation due to dehydration and partial small bowel obstruction. An intervention of a patient orientated educational card was provided to all patients undergoing a new loop ileostomy formation to educate them on monitoring ileostomy output, do's and don'ts of appropriate foods and what to do if concerns regarding ileostomy output. The current status of this project is in the progress of analysis of outcomes after the intervention to close the audit cycle loop. 


\section{Research}

\section{Peer Reviewed Publications}

Galbraith NJ, Walker SP, Carter JV, Polk HC. Past, present and future of augmentation of monocyte function in the surgical patient. Surgical Infections. 2016 Surg Infect (Larchmt). 2016 Oct;17(5):563-9.

Carter JV, Roberts HL, Pan J, Rice JD, Burton JF, Galbraith NJ, Eichenberger MR, Jorden J, Deveaux P, Farmer R, Williford A, Kanaan Z, Rai SN, Galandiuk S. A Highly Predictive Model for Diagnosis of Colorectal Neoplasms Using Plasma MicroRNA: Improving Specificity and Sensitivity. Ann Surg. 2016 Oct;264(4):575-84.

Galbraith NJ, Walker SP, Galandiuk S, Gardner SA, Polk HC. The significance and challenges of monocyte impairment: for the ill patient and the surgeon. Surgical Infections. 2016 vol. 17 (3) pp 303-12.

Billeter AT, Galbraith N, Walker S, Lawson C, Gardner SA, Sarojini H, Galandiuk S, Polk HC. TRPA1 mediates the effects of hypothermia on the monocyte inflammatory response. Surgery. 2015 Sep 30;158(3):646-54.

Galbraith NJ, Cullis PS, O’Dwyer PJ. Letter to the Editor: Minimally invasive surgery for malignant adrenal tumors. The surgeon. 2014 Apr 30;12(115):e116-116.

Parnaby CN, Galbraith N, O'Dwyer PJ. Importance of the Adrenal Gland Blood Supply During Laparoscopic Subtotal Adrenalectomy. J Laparoendosc Adv Surg Tech A. 2010 Mar 8

Parnaby CN, Galbraith N, O'Dwyer PJ. Experience in identifying the venous drainage of the adrenal gland during laparoscopic adrenalectomy. Clin Anat. 2008 Oct; 21(7): 660-5.

\section{Papers in progress:}

Galbraith NJ, Walker SP, Cahill M, Bishop C, Manek S, Reid M, Polk HC. Enhancing impaired host defense mechanisms: An immunological basis for adjuvant therapies.

Submitted to Journal of Leukocyte. Biology, December 2016

Galbraith NJ, Carter JV, Netz U, McCafferty M, Yang D, Fry DE, Galandiuk S. A contemporary metaanalysis of laparoscopic lavage for perforated diverticulitis.

Submitted to JAMA Surgery, November 2016

Walker SP, Galbraith NJ, Bishop C, Reid M, Gardner SA, Polk HC. Interferon-gamma enhances monocyte function and increases PD-L1 expression.

Accepted for oral presentation at ASC, 2017, Las Vegas. Target journal: PLoS One.

Galbraith NJ, Walker SP, Carter JV, Gardner SA, Galandiuk S, Polk HC. The role and regulation of I $x$ K in relation to impaired monocyte function.

Accepted for presentation at ASC, 2017, Las Vegas. Target journal: Surgery

Galbraith NJ, Walker SP, Gardner SA, Carter JV, Manek S, Galandiuk S, Polk HC. Surgical patients with infection have suppressed monocyte function and increased IRAK-M expression. Accepted for presentation at ASC, 2017, Las Vegas. Target journal: Journal of Immunology

Galbraith NJ, Walker SP, Carter JV, Gardner SA, Galandiuk S, Smith JW, Barnes SL, Polk HC. Major trauma causes microRNA changes associated with pro-inflammatory cytokine signaling. Target journal: Annals of Surgery.

Galbraith NJ, Netz U, Carter JV, Polk HC Jr., Galandiuk S. Long term outcomes in patients diagnosed with Indeterminate Colitis - a retrospective case review.

"Radiofrequency ablation for breast cancer liver metastasis: A case of tumour progression"

Galbraith NJ, Wigmore SJ. 
"MBL and the acute phase response: A factor in post-operative infection following liver resection" Galbraith NJ, Wigmore SJ.

"Key Factors affecting Compensatory Renal Hypertrophy following Live Kidney Donation" Galbraith NJ, Clancy MJ.

\section{Presentations}

Poster presentation, Surgical Infection Society Annual Meeting, West Palm Beach, FL. May 2016.

"Dysregulation of NFxB in the impaired human monocyte leads to a blunted TNF- $\alpha$ response"

Oral presentation, Academic Surgical Congress, Jacksonville, FL. February 2016 (First Author \& Presenter) "MicroRNA dysregulation associated with function of the impaired monocyte"

Price Seminar, Price Institute of Surgical Research, Department of Surgery, University of Louisville, March 2015

"Drug prophylaxis and other adjunctive therapies for septic complications in the surgical patient"

Oral presentation, Academic Surgical Congress, Las Vegas February 2015 (First Author \& Presenter) "TRPA1 mediates the effects of hypothermia on the monocyte inflammatory response"

Oral presentation (Vascular/Transplant Section), Association of Surgeons of Great Britain \& Ireland, Harrogate, 2014 (First Author \& Presenter)

"Factors affecting Compensatory Renal Hypertrophy following Live Kidney Donation"

Poster, Association of Surgeons of Great Britain \& Ireland, Harrogate, 2014 (First Author \& Presenter) "MBL and the acute phase response: A factor in post-operative infection following liver resection."

Poster, Association of Surgeons in Training, 2014 (First Author \& Presenter)

"Key Factors affecting Compensatory Renal Hypertrophy following Live Kidney Donation"

ePoster, ESSQ MSc, University of Edinburgh, 2013 (First Author \& Presenter)

"MBL and the acute phase response: A factor in post-operative infection following liver resection"

Poster, European Society for Coloprotology (ESCP), Vienna, 2012 (First Author)

"Fluid prescription in Surgical Patients by Junior Doctors"

Poster, European Society of Organ Transplantation (ESOT), 2011 (Third Author)

"Compensatory Renal Hypertrophy Following Laparoscopic Live Kidney Donation: No Reduction

Compared with Open Techniques"

Poster, ASGBI International Surgical Congress, 2010 (Second Author)

"Patient Outcome Following Laparoscopic Adrenalectomy for Adrenal Metastasis"

Poster, British Association of Endocrine and Thyroid Surgeons Annual Meeting, 2009 (Presenter and First Author)

"Laparoscopic adrenalectomy in metastatic renal cell carcinoma."

Oral presentation, Scottish Renal Association Meeting, 2009 (Presenter and First Author)

"Renal function in patients following live donor nephrectomy."

Oral presentation at the Winter Meeting of the British Association of Clinical Anatomists, 2007 (Second Author)

"Importance of adrenal vein anatomy during laparoscopic adrenalectomy."

\section{Book Chapters}

Galbraith NJ, Wiemann TJ, Polk HC.

The Prevention and Treatment of the Diabetic Foot Fischer's Mastery of Surgery, 7th Edition. 


\section{Book Reviews}

"How to Pass the MRCS OSCE Volume 1 (Oxford Specialty Training: Revision Texts)" by Pradip K.

Datta, Chris Bulstrode, Vasha Kaur

Reviewed by P. Cullis \& NJ. Galbraith

Published at British Journal of Surgery (BJS) Online: Book Reviews

"Fundamentals of Surgical Practice ( $3^{\text {rd }}$ Edition) by Andrew Kingsnort and Douglas Boweley

Reviewed by P. Cullis \& NJ. Galbraith

Published 24 ${ }^{\text {th }}$ Feb 2014 in Surgeons News, Royal College of Surgeons, Edinburgh

\section{Teaching}

I am registered for my third consecutive year as an eTutor for the Edinburgh Surgical Sciences

Qualification MSc programme, teaching junior doctors in the Colorectal \& Urology modules through Case Based Discussion enforcing basic science principles and formally assessing them (2013 - present).

I have carried out teaching to medical students including bed side teaching, lectures and interactive tutorials.

There has been ample opportunity to teach within the academic foundation programme. Recent teaching sessions included:

- Surgical emergencies for the FY1

- Topics in Gastroenterology (3rd year medical students) including inflammatory bowel disease, upper GI bleeds and colorectal cancer.

In my recent clinical year before leaving Edinburgh, I was an examiner in the $3^{\text {rd }}$ year OSCE's for their GI block with the University of Edinburgh.

Since my time in Louisville, I have regularly taken part as an examiner in the 3rd year medical student surgical oral exams, as well as regular attendance on Dr. Polk' teaching trauma rounds.

\section{Management, Leadership \& Teamwork}

Following on from heavy involvement in the audit process, following a successful presentation of our VTE audit in the Western Isles Hospital, I attended meetings with the Medicines Management team to establish new guidelines based on our audit, which was highly successful.

Since then, I have organized X-Ray meetings, the Stone meeting in my current Urology rotation, and recently presented a paper at our departmental Journal Club in the Colorectal Surgery.

As a research fellow in the Price Institute of Surgical Research, I was responsible for up to 5 students in the lab at any time. This involved leadership in pioneering and delegating individual projects, supervising experimental technique, assisting in data analysis and presentation of results. Leadership of this team provided a challenge in ensuring all members of the team benefited. This was a highly successful endeavor resulting in a medical student presenting at the Research Louisville symposium (2016) and a prospective medical student presenting our work at national level. This role also involved the organising the weekly lab meeting and coordinating with other research fellows and lab staff to ensure successful collaboration. 


\section{Non - Academic Achievements}

- Louisville Half Marathon 2016

- Edinburgh Marathon 2012

- Loch Ness Marathon 2010

- Students for Kids International Projects (SKIP), Llongwe, Malawi 2008

- Mount Kilimanjaro, Tanzania 2008

- 50/50 Charity Event, University of Glasgow 2006

- Certificate of Excellence for Selection in Home International Hill Race 2004 Giffnock North Athletics Club

- Level 1 Athletics Coaching Certificate, UK Athletics

\section{Interests}

I feel it is important to balance a busy work and academic life with social and physical activities. In particular, I train and compete regularly in distance running. This has been at school, club, university and international levels over the past 10 years. I have broadened my range of running to include both hill running such as the Kintyre Way Relay, and long distance running including the Loch Ness Marathon. 